\title{
Nickel-Catalyzed Desymmetric Hydrogenation of Cyclohexadienones: An Efficient Approach to All-Carbon Quaternary Stereocenters
}

[Supporting Information]

Cai You, ${ }^{\dagger a}$ Xiuxiu Li, ${ }^{\dagger a}$ Quan Gong, ${ }^{a}$ Jialin Wen, ${ }^{* a, b}$ and Xumu Zhang*a,c

${ }^{a}$ Department of Chemistry, Southern University of Science and Technology, 1088 Xueyuan Road, Shenzhen, 518055, China.

${ }^{\mathrm{b}}$ Academy of Advanced Interdisciplinary Studies, Southern University of Science and Technology, 1088 Xueyuan Road, Shenzhen, 518055, China

${ }^{c}$ Shenzhen Grubbs Institute, Southern University of Science and Technology, 1088 Xueyuan Road, Shenzhen, 518055, China

\section{Table of Contents}

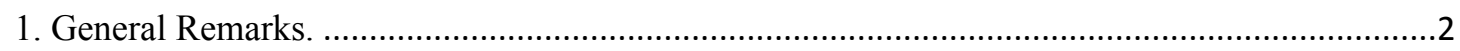

2. General Procedure for the Synthesis of $\gamma, \gamma$-disubstituted Cyclohexadienone................................

3. General Procedure for Asymmetric Hydrogenation of 1 and 3.................................................

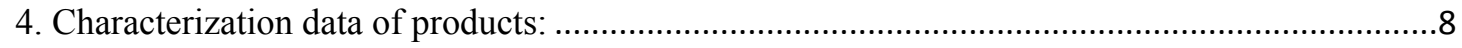

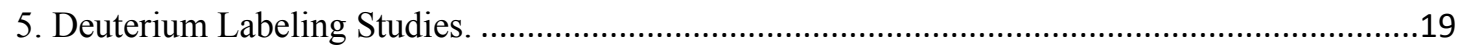

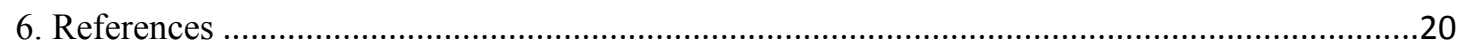

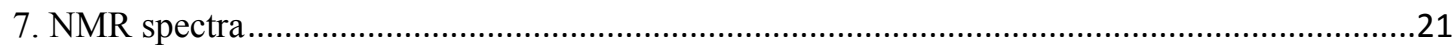

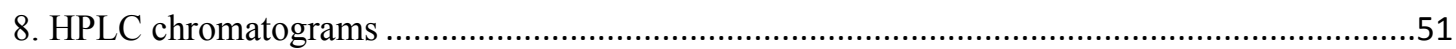




\section{General Remarks.}

All the reactions dealing with air- or moisture- sensitive compounds were carried out in a dry reaction vessel under an argon atmosphere or in an argon-filled glove box. Unless otherwise noted, all reagents and solvents were purchased from commercial suppliers without further purification. Toluene was dried with sodium chips and indicated by benzophenone. Other anhydrous solvents were purchased from J\&K Chemical and degassed by bubbling argon over a period of $30 \mathrm{~min}$. Purification of products was carried out by flash chromatography using silica gel (200-300 mesh). Thin layer chromatography (TLC) was performed on EM reagents $0.25 \mathrm{~mm}$ silica $60-\mathrm{F}$ plates. $\mathrm{Ni}(\mathrm{OTf})_{2}$ and other metal precursors were purchased from Strem.

${ }^{1} \mathrm{H},{ }^{13} \mathrm{C},{ }^{19} \mathrm{~F}$ and ${ }^{31} \mathrm{P}$ NMR spectra were recorded on a Bruker Avance $400 \mathrm{MHz}$ or a Bruker Avance $600 \mathrm{MHz}$ spectrometer with tetramethylsilane as the internal standard. Chemical shifts are reported in parts per million (ppm, $\delta$ scale) downfield from TMS at $0.00 \mathrm{ppm}$ and referenced to the $\mathrm{CDCl}_{3}$ at $7.27 \mathrm{ppm}$ for ${ }^{1} \mathrm{H}$ NMR or $77.0 \mathrm{ppm}$ for ${ }^{13} \mathrm{C} \quad$ NMR. Data are reported as: multiplicity $(\mathrm{s}=$ singlet, $\mathrm{d}=$ doublet, $\mathrm{t}=$ triplet, $\mathrm{q}=$ quartet, $\mathrm{m}=$ multiplet $)$, coupling constant in hertz $(\mathrm{Hz})$ and signal area integration in natural numbers. ${ }^{13} \mathrm{C}$ NMR and ${ }^{31} \mathrm{P}$ NMR analyses were recorded with ${ }^{1} \mathrm{H}$ decoupling. Enantiomeric excess values were determined with Agilent 1290 or 1260 Series HPLC instrument on a chiral stationary phase. Optical rotations were measured using a $1 \mathrm{~mL}$ cell with a $1 \mathrm{dm}$ path length on a Rudolph Autopol I polarimeter at $589 \mathrm{~nm}$. All new products were further characterized by HRMS. A positive ion mass spectrum of sample was acquired on a Thermo LTQ-FT mass spectrometer with an electrospray ionization source. 


\section{General Procedure for the Synthesis of $\gamma, \gamma$-disubstituted Cyclohexadienone.}

Method A: (for compound 1a, 3f)<smiles>[R]C(=O)C([R])[R]</smiles>

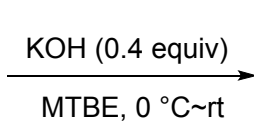<smiles>[R]C1([R])C=CC(=O)CC1</smiles><smiles>[R]C1([R10])C=CCC=C1</smiles>

Methyl vinyl ketone $(2.1 \mathrm{~g}, 30 \mathrm{mmol})$, aldehyde (30 mmol), and methyl tert-buthyl ether (MTBE) $(100 \mathrm{~mL})$ were added to a $250 \mathrm{~mL}$ round bottom flask. The reaction mixture was cooled to $0{ }^{\circ} \mathrm{C}$ and an ethanolic solution of $\mathrm{KOH}(0.7 \mathrm{~g}$ in $7.5 \mathrm{~mL}$ ethanol, $12 \mathrm{mmol})$ was added slowly over $15 \mathrm{~min}$ and allowed to stir for $2 \mathrm{~h}$ at the same temperature. The reaction mixture was warmed to room temperature and stirred for additional $1 \mathrm{~h}$. The reaction mixture was washed with $1 \mathrm{M} \mathrm{HCl}(30 \mathrm{~mL})$, water $(30 \mathrm{~mL})$, and brine $(30 \mathrm{~mL})$. The organic layer was dried over anhydrous $\mathrm{Na}_{2} \mathrm{SO}_{4}$, filtered through Celite and the volatiles were removed in vacuo to afford the crude cyclohexenone. The resultant mixture was purified by flash column chromatography to furnish racemic cyclohexenone.

Cyclohexenone (21 mmol), DDQ (6.5 g, $28 \mathrm{mmol})$, and 1,4-dioxane $(50 \mathrm{~mL})$ were added to a 3 neck, $100 \mathrm{~mL}$ round bottom flask equipped with reflux condenser. The reaction mixture was heated to $100{ }^{\circ} \mathrm{C}$ and stirred for $22 \mathrm{~h}$. The reaction mixture was cooled down to room temperature and filtered through Celite. The filtrates were diluted with methyl tert-buthyl ether (MTBE), washed with 5\% aqueous $\mathrm{NaOH}$, and water sequentially. The organic layer was dried over anhydrous $\mathrm{MgSO}_{4}$, filtered through Celite and the volatiles were removed in vacuo to afford the crude cyclohexadienone. The resultant mixture was purified by flash column chromatography to furnish cyclohexadienone.

Method B: (for other compounds except for 1a, 3f)
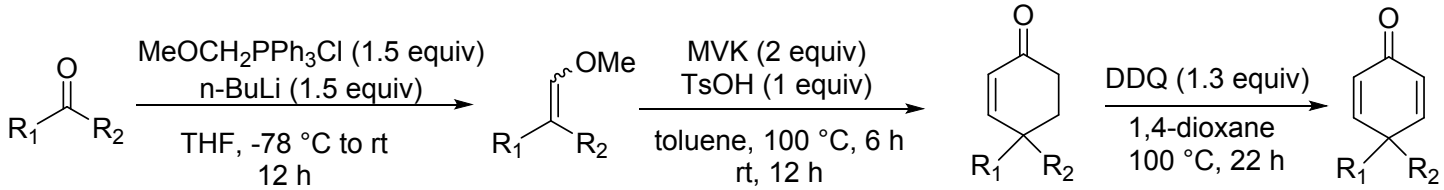

(Methoxymethyl)triphenylposhponium chloride (10.3 g, $30.0 \mathrm{mmol})$ and THF (200 mL) were added to a $500 \mathrm{~mL} 3$ neck round bottom flask and cooled to $-78^{\circ} \mathrm{C} . \mathrm{n}-\mathrm{BuLi}(2.0 \mathrm{M}$ in hexane, $15 \mathrm{~mL}, 30 \mathrm{mmol})$ was added slowly to the reaction mixture and stirred for $1 \mathrm{~h}$ at $-78{ }^{\circ} \mathrm{C}$ and additional $1 \mathrm{~h}$ at room 
temperature. The reaction mixture was cooled to $-78^{\circ} \mathrm{C}$. Ketone $(20 \mathrm{mmol})$ in $\mathrm{THF}(40 \mathrm{~mL}) \mathrm{was}$ added slowly to the mixture, which was stirred for $30 \mathrm{~min}$ at the same temperature. The reaction mixture was warmed to room temperature and stirred for $12 \mathrm{~h}$. The reaction mixture was quenched with water and extracted into EtOAc (3X). The combined organic layers were washed with saturated brine solution, dried over anhydrous $\mathrm{MgSO}_{4}$, filtered through Celite and concentrated in vacuo to afford the crude enol ether. The resultant mixture was purified by flash column chromatography to provide enol ethers.

Enol ether $(20 \mathrm{mmol})$ and p-toluenesulfonic acid monohydrate $(4.0 \mathrm{~g}, 21 \mathrm{mmol})$, and toluene $(70 \mathrm{~mL})$ were placed in a $250 \mathrm{~mL}$ flask. Methyl vinyl ketone $(40 \mathrm{mmol})$ was added to the mixture, which was stirred at $100{ }^{\circ} \mathrm{C}$ for $6 \mathrm{~h}$. The reaction mixture was allowed to cool to room temperature and stirring for $12 \mathrm{~h}$. The mixture was quenched with saturated aqueous $\mathrm{NaHCO}_{3}$. The mixture was extracted with EtOAc (3X). The combined organic layers were dried over anhydrous $\mathrm{MgSO}_{4}$, filtered through Celite and concentrated in vacuo to afford the crude cyclohexenone. The resultant mixture was purified by flash column chromatography to provide racemic cyclohexenone.

Cyclohexenone (15 mmol), DDQ (4.6 g, $20 \mathrm{mmol})$, and 1,4-dioxane (36 mL) were added to a 3 neck, $100 \mathrm{~mL}$ round bottom flask equipped with reflux condenser. The reaction mixture was heated to $100{ }^{\circ} \mathrm{C}$ and stirred for $22 \mathrm{~h}$. The reaction mixture was cooled down to room temperature and filtered through Celite. The filtrates were diluted with methyl tert-buthyl ether (MTBE), washed with 5\% aqueous $\mathrm{NaOH}$, and water sequentially. The organic layer was dried over anhydrous $\mathrm{MgSO}_{4}$, filtered through Celite and the volatiles were removed in vacuo to afford the crude cyclohexadienone. The resultant mixture was purified by flash column chromatography to furnish cyclohexadienone.

For new compounds:<smiles>CC1(c2ccc(F)cc2)C=CC(=O)C=C1</smiles>

4'-fluoro-1-methyl-[1,1'-biphenyl]-4(1H)-one (1e): white solid; ${ }^{1} \mathrm{H}$ NMR (400 MHz, $\left.\mathrm{CDCl}_{3}\right) \delta: 7.28$ (ddd, $J=7.0,5.2,2.6 \mathrm{~Hz}, 2 \mathrm{H}), 7.08-7.00(\mathrm{~m}, 2 \mathrm{H}), 6.93-6.86(\mathrm{~m}, 2 \mathrm{H}), 6.33-6.25(\mathrm{~m}, 2 \mathrm{H}), 1.69(\mathrm{~s}, 3 \mathrm{H})$. ${ }^{13} \mathrm{C}$ NMR $\left(101 \mathrm{MHz}, \mathrm{CDCl}_{3}\right) \delta: 186.0,162.3(\mathrm{~d}, J=247.1 \mathrm{~Hz}), 155.4,135.9,128.3(\mathrm{~d}, J=8.1 \mathrm{~Hz}), 127.3$, 
$116.1(\mathrm{~d}, J=21.3 \mathrm{~Hz}), 44.7,24.3 .{ }^{19} \mathrm{~F} \mathrm{NMR}\left(376 \mathrm{MHz}, \mathrm{CDCl}_{3}\right) \delta-114.4 . \mathbf{H R M S}$ calculated $[\mathrm{M}+\mathrm{H}]^{+}$for $\mathrm{C}_{13} \mathrm{H}_{11} \mathrm{FO}=203.0867$, found: 203.0865 .<smiles>CC1(c2ccc(Br)cc2)C=CC(=O)C=C1</smiles>

4'-bromo-1-methyl-[1,1'-biphenyl]-4(1H)-one (1g): white solid; ${ }^{1} \mathrm{H}$ NMR (400 MHz, $\left.\mathrm{CDCl}_{3}\right) \delta$ : 7.51 $7.44(\mathrm{~m}, 2 \mathrm{H}), 7.22-7.14(\mathrm{~m}, 2 \mathrm{H}), 6.91-6.84(\mathrm{~m}, 2 \mathrm{H}), 6.29(\mathrm{dd}, \mathrm{J}=8.9,1.2 \mathrm{~Hz}, 2 \mathrm{H}), 1.67(\mathrm{~s}, 3 \mathrm{H}) .{ }^{13} \mathrm{C}$ $\operatorname{NMR}\left(101 \mathrm{MHz}, \mathrm{CDCl}_{3}\right) \delta: 185.9,154.9,139.3,132.4,128.5,127.6,122.0,44.9,24.1$. HRMS calculated $[\mathrm{M}+\mathrm{H}]^{+}$for $\mathrm{C}_{13} \mathrm{H}_{11} \mathrm{BrO}=263.0066$, found: 263.0065 .<smiles>COc1ccc(C2(C)C=CC(=O)C=C2)cc1OC</smiles>

3',4'-dimethoxy-1-methyl-[1,1'-biphenyl]-4(1H)-one (1j): yellow solid; ${ }^{1} \mathrm{H}$ NMR (400 MHz, $\mathrm{CDCl}_{3}$ ) $\delta: 6.93-6.83(\mathrm{~m}, 4 \mathrm{H}), 6.74(\mathrm{~d}, J=2.0 \mathrm{~Hz}, 1 \mathrm{H}), 6.27(\mathrm{dt}, J=4.8,3.0 \mathrm{~Hz}, 2 \mathrm{H}), 3.87(\mathrm{~s}, 3 \mathrm{H}), 3.84(\mathrm{~s}, 3 \mathrm{H})$, $1.68(\mathrm{~s}, 3 \mathrm{H}) .{ }^{13} \mathrm{C}$ NMR $\left(101 \mathrm{MHz}, \mathrm{CDCl}_{3}\right) \delta: 186.0,155.7,149.2,148.6,132.1,126.8,118.4,111.4$, 109.9, 56.0, 55.9, 44.7, 23.9. HRMS calculated $[\mathrm{M}+\mathrm{H}]^{+}$for $\mathrm{C}_{15} \mathrm{H}_{16} \mathrm{O}_{3}=245.1172$, found: 245.1170 .<smiles>CCC1(C2CCCCC2)C=CC(=O)C=C1</smiles>

1-ethyl-[1,1'-bi(cyclohexane)]-2,5-dien-4-one (1m): colorless oil; ${ }^{1} \mathrm{H}$ NMR $\left(400 \mathrm{MHz}, \mathrm{CDCl}_{3}\right) \delta$ : 6.78 $6.71(\mathrm{~m}, 2 \mathrm{H}), 6.43-6.31(\mathrm{~m}, 2 \mathrm{H}), 1.81-1.64(\mathrm{~m}, 7 \mathrm{H}), 1.53(\mathrm{tt}, J=12.2,2.9 \mathrm{~Hz}, 1 \mathrm{H}), 1.26-1.15(\mathrm{~m}, 2 \mathrm{H})$, 1.13-1.04 (m, 1H), $0.98(\mathrm{tt}, J=12.5,6.2 \mathrm{~Hz}, 2 \mathrm{H}), 0.71(\mathrm{t}, J=7.5 \mathrm{~Hz}, 3 \mathrm{H}) .{ }^{13} \mathrm{C} \mathrm{NMR}\left(101 \mathrm{MHz}, \mathrm{CDCl}_{3}\right)$ $\delta: 187.3,154.8,130.9,49.8,46.3,29.4,28.3,27.0,26.6,8.9$. HRMS calculated $[\mathrm{M}+\mathrm{H}]^{+}$for $\mathrm{C}_{14} \mathrm{H}_{20} \mathrm{O}=$ 205.1587, found: 205.1586 . 
<smiles>O=C1C=CC2(C=C1)CCCc1sccc12</smiles>

6,7-dihydro-5H-spiro[benzo[b]thiophene-4,1'-cyclohexane]-2',5'-dien-4'-one (3i): yellow solid; ${ }^{1} \mathrm{H}$ NMR (400 MHz, $\left.\mathrm{CDCl}_{3}\right) \delta: 7.03(\mathrm{~d}, J=5.2 \mathrm{~Hz}, 1 \mathrm{H}), 6.97-6.90(\mathrm{~m}, 2 \mathrm{H}), 6.54(\mathrm{~d}, J=5.2 \mathrm{~Hz}, 1 \mathrm{H}), 6.30-$ $6.23(\mathrm{~m}, 2 \mathrm{H}), 2.91(\mathrm{t}, J=6.2 \mathrm{~Hz}, 2 \mathrm{H}), 2.09-2.03(\mathrm{~m}, 2 \mathrm{H}), 1.95-1.91(\mathrm{~m}, 2 \mathrm{H}) .{ }^{13} \mathrm{C}$ NMR $(101 \mathrm{MHz}$, $\left.\mathrm{CDCl}_{3}\right) \delta: 186.3,154.4,137.9,132.2,127.5,126.5,122.9,43.4,33.6,24.9,20.2$ ppm. HRMS calculated $[\mathrm{M}+\mathrm{H}]^{+}$for $\mathrm{C}_{13} \mathrm{H}_{12} \mathrm{OS}=217.0682$, found: 217.0680 . 


\section{General Procedure for Asymmetric Hydrogenation of 1 and 3.}

\section{Procedure for condition optimization:}

A stock solution was made by mixing $\mathrm{Ni}(\mathrm{OTf})_{2}(3.57 \mathrm{mg}, 0.01 \mathrm{mmol})$ with $(S, S)-\mathrm{Ph}-\mathrm{BPE}(5.6 \mathrm{mg}$, $0.011 \mathrm{mmol})$ in a $1: 1.1$ molar ratio in $\mathrm{MeOH} /$ toluene $(1: 1,1 \mathrm{ml})$ at room temperature for $30 \mathrm{~min}$ in a nitrogen-filled glovebox. An aliquot of the catalyst solution $(0.1 \mathrm{~mL}, 0.001 \mathrm{mmol})$ was transferred by syringe into the vials charged with 1a $(0.1 \mathrm{mmol}$ for each $)$ in anhydrous toluene $(0.9 \mathrm{~mL})$. The vials were subsequently transferred into an autoclave into which hydrogen gas was charged. The reaction was then stirred under $\mathrm{H}_{2}(50 \mathrm{~atm})$ at $50{ }^{\circ} \mathrm{C}$ for $24 \mathrm{~h}$. After cooling to room temperature, the hydrogen gas was released slowly and carefully. The solution was concentrated and passed through a short column of silica gel (eluant: EtOAc) to remove the metal complex. The ee values of all compounds were determined by HPLC analysis on a chiral stationary phase.

\section{Procedure for substrate scope:}

A stock solution was made by mixing $\mathrm{Ni}(\mathrm{OTf})_{2}(3.57 \mathrm{mg}, 0.01 \mathrm{mmol})$ with $(S, S)-\mathrm{Ph}-\mathrm{BPE}(5.6 \mathrm{mg}$, $0.011 \mathrm{mmol})$ in a $1: 1.1 \mathrm{molar}$ ratio in $\mathrm{MeOH} /$ toluene $(1: 1,1 \mathrm{ml})$ at room temperature for $30 \mathrm{~min}$ in a nitrogen-filled glovebox. An aliquot of the catalyst solution $(0.1 \mathrm{~mL}, 0.001 \mathrm{mmol})$ was transferred by syringe into the vials charged with different substrates $(0.1 \mathrm{mmol}$ for each) in anhydrous toluene $(0.9$ $\mathrm{mL}$ ). The vials were subsequently transferred into an autoclave into which hydrogen gas was charged. The reaction was then stirred under $\mathrm{H}_{2}(50 \mathrm{~atm})$ at $50{ }^{\circ} \mathrm{C}$ for $24 \mathrm{~h}$. After cooling to room temperature, the hydrogen gas was released slowly and carefully. The solution was concentrated and the crude product was purified by flash chromatography (on silica, eluent: EtOH/Petroleum ether). The ee values of all compounds were determined by HPLC analysis on a chiral stationary phase.

Racemic samples were obtained during the synthesis of substrates before the final oxidation step, please refer to the section two (page S3).

The absolute configurations of the known products were assigned by the comparison of the optical rotation with the known values in literature, the others were determined by analogy. 


\section{Characterization data of products:}<smiles>C[C@]1(c2ccccc2)C=CC(=O)CC1</smiles>

(S)-1-methyl-2,3-dihydro-[1,1'-biphenyl]-4(1H)-one (2a): colorless oil; isolated yield: 96\%; 93\% ee; $[\alpha]_{\mathrm{D}}{ }^{24}=-70.4(\mathrm{c}=0.5, \mathrm{EtOH}) ;\left[\right.$ lit. ${ }^{1}$ for the opposite enantiomer $[\alpha]_{\mathrm{D}}{ }^{23}=+110.1(\mathrm{c}=0.7, \mathrm{EtOH})$ for $93 \%$ ee]; The enantiomeric excess was determined by HPLC on Chiralpak AD-3 column, hexane: isopropanol $=97: 3$; flow rate $=1.0 \mathrm{~mL} / \mathrm{min} ; \mathrm{UV}$ detection at $220 \mathrm{~nm} ; \mathrm{t}_{\mathrm{R}}=6.9 \mathrm{~min}$ (major), $7.8 \mathrm{~min}$ (minor). ${ }^{1} \mathrm{H}$ NMR $\left(400 \mathrm{MHz}, \mathrm{CDCl}_{3}\right) \delta: 7.38-7.33(\mathrm{~m}, 4 \mathrm{H}), 7.29-7.25(\mathrm{~m}, 1 \mathrm{H}), 6.94(\mathrm{dd}, J=10.2,0.8 \mathrm{~Hz}, 1 \mathrm{H}), 6.13(\mathrm{~d}, J=$ $10.2 \mathrm{~Hz}, 1 \mathrm{H}), 2.45-2.37 \mathrm{~m}, 1 \mathrm{H}), 2.33-2.23(\mathrm{~m}, 2 \mathrm{H}), 2.18-2.11(\mathrm{~m}, 1 \mathrm{H}), 1.57(\mathrm{~s}, 3 \mathrm{H}) .{ }^{13} \mathrm{C}$ NMR (101 MHz, $\left.\mathrm{CDCl}_{3}\right) \delta: 199.8,157.4,145.5,128.9,128.8,127.1,126.4,40.9,38.4,34.9,27.9$.<smiles>Cc1ccc([C@]2(C)C=CC(=O)CC2)cc1</smiles>

(S)-1,4'-dimethyl-2,3-dihydro-[1,1'-biphenyl]-4(1H)-one (2b): yellow oil; isolated yield: 98\%; 94\% ee; $[\alpha]_{D}{ }^{24}=-105.0(\mathrm{c}=0.5, \mathrm{EtOH}) ;\left[\right.$ lit. $^{2}$ for the opposite enantiomer $[\alpha]_{\mathrm{D}}{ }^{25}=+110.8(\mathrm{c}=1.3, \mathrm{MeOH})$ for $91 \%$ ee]; The enantiomeric excess was determined by HPLC on Chiralpak AD-3 column, hexane: isopropanol $=97: 3$; flow rate $=1.0 \mathrm{~mL} / \mathrm{min} ; \mathrm{UV}$ detection at $220 \mathrm{~nm} ; \mathrm{t}_{\mathrm{R}}=6.2 \min$ (major), $6.9 \min$ (minor). ${ }^{1} \mathrm{H} \mathrm{NMR}\left(400 \mathrm{MHz}, \mathrm{CDCl}_{3}\right) \delta: 7.22(\mathrm{~d}, J=8.3 \mathrm{~Hz}, 2 \mathrm{H}), 7.16(\mathrm{~d}, J=8.2 \mathrm{~Hz}, 2 \mathrm{H}), 6.92(\mathrm{~d}, J=$ $10.2 \mathrm{~Hz}, 1 \mathrm{H}), 6.11(\mathrm{~d}, J=10.2 \mathrm{~Hz}, 1 \mathrm{H}), 2.43-2.36(\mathrm{~m}, 1 \mathrm{H}), 2.33(\mathrm{~s}, 3 \mathrm{H}), 2.31-2.27$ (m, 1H), 2.26-2.21 (m, 1H), 2.15-2.08 (m, 1H), $1.54(\mathrm{~s}, 3 \mathrm{H}) .{ }^{13} \mathrm{C}$ NMR $\left(101 \mathrm{MHz}, \mathrm{CDCl}_{3}\right) \delta: 199.8,157.6,142.4,136.6$, $129.5,128.6,126.3,40.5,38.3,34.9,27.9,21.1$.<smiles>COc1ccc([C@]2(C)C=CC(=O)CC2)cc1</smiles> 
(S)-4'-methoxy-1-methyl-2,3-dihydro-[1,1'-biphenyl]-4(1H)-one (2c): yellow oil; isolated yield: 94\%; $93 \%$ ee; $[\alpha]_{\mathrm{D}}{ }^{24}=-98.2(\mathrm{c}=0.5, \mathrm{EtOH}) ;\left[\right.$ lit. $^{3}$ for the opposite enantiomer $[\alpha]_{\mathrm{D}}{ }^{26}=+135.6(\mathrm{c}=1.0, \mathrm{EtOH})$ for 91\% ee]; The enantiomeric excess was determined by HPLC on Chiralpak OD-3 column, hexane: isopropanol $=97: 3$; flow rate $=1.0 \mathrm{~mL} / \mathrm{min} ; \mathrm{UV}$ detection at $210 \mathrm{~nm} ; \mathrm{t}_{\mathrm{R}}=9.4 \mathrm{~min}$ (major), $10.3 \mathrm{~min}$ (minor). ${ }^{1} \mathrm{H}$ NMR (400 MHz, $\left.\mathrm{CDCl}_{3}\right)$ $\delta: ~ 7.28-7.24(\mathrm{~m}, 2 \mathrm{H}), 6.93-6.87(\mathrm{~m}, 3 \mathrm{H}), 6.11(\mathrm{~d}, J=10.2 \mathrm{~Hz}, 1 \mathrm{H})$, $3.80(\mathrm{~s}, 3 \mathrm{H}), 2.43-2.36(\mathrm{~m}, 1 \mathrm{H}), 2.34-2.25(\mathrm{~m}, 1 \mathrm{H}), 2.25-2.18(\mathrm{~m}, 1 \mathrm{H}), 2.15-2.08(\mathrm{~m}, 1 \mathrm{H}), 1.54(\mathrm{~s}, 3 \mathrm{H})$.

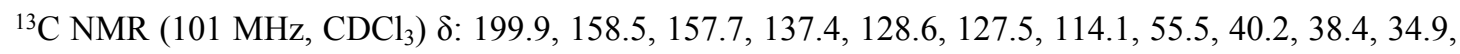
28.0.<smiles>C[C@]1(c2ccc(C(F)(F)F)cc2)C=CC(=O)CC1</smiles>

(S)-1-methyl-4'-(trifluoromethyl)-2,3-dihydro-[1,1'-biphenyl]-4(1H)-one (2d): yellow oil; isolated yield: $95 \%$; $97 \%$ ee; $[\alpha]_{\mathrm{D}}^{24}=-66.0(\mathrm{c}=0.5, \mathrm{EtOH}) ;\left[\right.$ lit. $^{2}$ for the opposite enantiomer $[\alpha]_{\mathrm{D}}{ }^{25}=+68.5(\mathrm{c}=$ 1.2, $\mathrm{MeOH}$ ) for $87 \%$ ee]; The enantiomeric excess was determined by HPLC on Chiralpak AD-3 column, hexane: isopropanol $=97: 3$; flow rate $=1.0 \mathrm{~mL} / \mathrm{min}$; $\mathrm{UV}$ detection at $220 \mathrm{~nm} ; \mathrm{t}_{\mathrm{R}}=7.0$ min (major), 8.0 min (minor). ${ }^{1} \mathrm{H}$ NMR (400 MHz, $\left.\mathrm{CDCl}_{3}\right) \delta: 7.62(\mathrm{~d}, J=8.3 \mathrm{~Hz}, 2 \mathrm{H}), 7.48(\mathrm{~d}, J=8.2 \mathrm{~Hz}, 2 \mathrm{H}), 6.93$ (dd, $J=10.2,0.7 \mathrm{~Hz}, 1 \mathrm{H}), 6.17(\mathrm{~d}, J=10.2 \mathrm{~Hz}, 1 \mathrm{H}), 2.50-2.39(\mathrm{~m}, 1 \mathrm{H}), 2.31-2.23(\mathrm{~m}, 2 \mathrm{H}), 2.23-2.17(\mathrm{~m}$, 1H), 1.60 (s, 3H). ${ }^{13} \mathrm{C} \mathrm{NMR}\left(101 \mathrm{MHz}, \mathrm{CDCl}_{3}\right) \delta: 199.0,156.0,149.7,129.3(\mathrm{q}, J=32.5 \mathrm{~Hz}), 129.3$, $126.9,125.8(\mathrm{q}, J=3.7 \mathrm{~Hz}), 124.3(\mathrm{q}, J=272.0 \mathrm{~Hz}), 40.9,38.2,34.7,27.7 .{ }^{19} \mathrm{~F} \mathrm{NMR}\left(376 \mathrm{MHz}, \mathrm{CDCl}_{3}\right)$ $\delta-63.01$.<smiles>C[C@]1(c2ccc(F)cc2)C=CC(=O)CC1</smiles>

(S)-4'-fluoro-1-methyl-2,3-dihydro-[1,1'-biphenyl]-4(1H)-one (2e): yellow oil; isolated yield: 97\%; $97 \%$ ee; $[\alpha]_{\mathrm{D}}{ }^{24}=-68.4(\mathrm{c}=0.5, \mathrm{EtOH})$; The enantiomeric excess was determined by HPLC on Chiralpak AD-3 column, hexane: isopropanol $=97: 3$; flow rate $=1.0 \mathrm{~mL} / \mathrm{min}$; $\mathrm{UV}$ detection at $210 \mathrm{~nm} ; \mathrm{t}_{\mathrm{R}}=7.8$ 
min (major), 8.5 min (minor). ${ }^{1} \mathrm{H}$ NMR (400 MHz, $\left.\mathrm{CDCl}_{3}\right) \delta:$ 7.35-7.28 (m, 2H), 7.09-6.99 (m, 2H), 6.91 $(\mathrm{dd}, J=10.2,0.7 \mathrm{~Hz}, 1 \mathrm{H}), 6.12(\mathrm{~d}, J=10.2 \mathrm{~Hz}, 1 \mathrm{H}), 2.46-2.38(\mathrm{~m}, 1 \mathrm{H}), 2.31-2.20(\mathrm{~m}, 2 \mathrm{H}), 2.17-2.10$ (m, 1H), 1.55 (s, 3H). ${ }^{13} \mathrm{C}$ NMR (101 MHz, $\left.\mathrm{CDCl}_{3}\right) \delta: 199.4,161.8(\mathrm{~d}, J=245.7 \mathrm{~Hz}), 156.9,141.2(\mathrm{~d}, J$ $=3.1 \mathrm{~Hz}), 128.9,128.02(\mathrm{~d}, J=7.9 \mathrm{~Hz}), 115.6(\mathrm{~d}, J=21.1 \mathrm{~Hz}), 40.4,38.4,34.8,27.9 .{ }^{19} \mathrm{~F}$ NMR $(376$ $\left.\mathrm{MHz}, \mathrm{CDCl}_{3}\right) \delta-116.52$. HRMS calculated $[\mathrm{M}+\mathrm{H}]^{+}$for $\mathrm{C}_{13} \mathrm{H}_{13} \mathrm{FO}=205.1023$, found: 205.1022 .<smiles>C[C@]1(c2ccc(Cl)cc2)C=CC(=O)CC1</smiles>

(S)-4'-fluoro-1-methyl-2,3-dihydro-[1,1'-biphenyl]-4(1H)-one (2f): yellow oil; isolated yield: 96\%; $95 \%$ ee; $[\alpha]_{\mathrm{D}}{ }^{24}=-89.0(\mathrm{c}=0.5, \mathrm{EtOH}) ;\left[\right.$ lit. $^{2}$ for the opposite enantiomer $[\alpha]_{\mathrm{D}}{ }^{25}=+99.6(\mathrm{c}=1.5, \mathrm{MeOH})$ for $86 \%$ ee]; The enantiomeric excess was determined by HPLC on Chiralpak AD-3 column, hexane: isopropanol $=97: 3$; flow rate $=1.0 \mathrm{~mL} / \mathrm{min} ; \mathrm{UV}$ detection at $210 \mathrm{~nm} ; \mathrm{t}_{\mathrm{R}}=8.1 \mathrm{~min}$ (major), $8.8 \mathrm{~min}$ (minor). ${ }^{1} \mathrm{H}$ NMR (400 MHz, $\left.\mathrm{CDCl}_{3}\right) \delta: 7.34-7.25(\mathrm{~m}, 4 \mathrm{H}), 6.89(\mathrm{dd}, J=10.2,0.8 \mathrm{~Hz}, 1 \mathrm{H}), 6.13(\mathrm{~d}, J=$ $10.2 \mathrm{~Hz}, 1 \mathrm{H}), 2.45-2.38(\mathrm{~m}, 1 \mathrm{H}), 2.30-2.19(\mathrm{~m}, 2 \mathrm{H}), 2.17-2.10(\mathrm{~m}, 1 \mathrm{H}), 1.55(\mathrm{~s}, 3 \mathrm{H}) .{ }^{13} \mathrm{C}$ NMR $(101$ $\left.\mathrm{MHz}, \mathrm{CDCl}_{3}\right) \delta: 199.24,156.52,144.07,132.91,129.05,128.96,127.86,40.51,38.29,34.73,27.80$.<smiles>CC1(c2ccc(Br)cc2)C=CC(=O)CC1</smiles>

(S)-4'-bromo-1-methyl-2,3-dihydro-[1,1'-biphenyl]-4(1H)-one (2g): yellow solid; isolated yield: 98\%; $95 \%$ ee; $[\alpha]_{\mathrm{D}}{ }^{24}=-88.6(\mathrm{c}=0.5, \mathrm{EtOH})$; The enantiomeric excess was determined by HPLC on Chiralpak AD-3 column, hexane: isopropanol $=97: 3$; flow rate $=1.0 \mathrm{~mL} / \mathrm{min} ; \mathrm{UV}$ detection at $210 \mathrm{~nm} ; \mathrm{t}_{\mathrm{R}}=8.6$ min (major), 9.4 min (minor). ${ }^{1} \mathrm{H}$ NMR (400 MHz, $\left.\mathrm{CDCl}_{3}\right) \delta:$ 7.52-7.40 (m, 2H), 7.24-7.19 (m, 2H), 6.89 $(\mathrm{dd}, J=10.2,0.8 \mathrm{~Hz}, 1 \mathrm{H}), 6.13(\mathrm{~d}, J=10.2 \mathrm{~Hz}, 1 \mathrm{H}), 2.47-2.37(\mathrm{~m}, 1 \mathrm{H}), 2.30-2.19(\mathrm{~m}, 2 \mathrm{H}), 2.18-2.10$ (m, 1H), 1.55 (s, 3H). ${ }^{13} \mathrm{C}$ NMR (101 MHz, $\left.\mathrm{CDCl}_{3}\right) \delta: 199.3,156.5,144.6,131.9,129.1,128.3,121.0$ 40.6, 38.3, 34.7, 27.8. HRMS calculated $[\mathrm{M}+\mathrm{H}]^{+}$for $\mathrm{C}_{13} \mathrm{H}_{13} \mathrm{BrO}=265.0223$, found: 265.0220. 
<smiles>COc1cccc([C@]2(C)C=CC(=O)CC2)c1</smiles>

(S)-3'-methoxy-1-methyl-2,3-dihydro-[1,1'-biphenyl]-4(1H)-one (2h): colorless oil; isolated yield: $94 \% ; 93 \%$ ee; $[\alpha]_{D}^{24}=-102.4(\mathrm{c}=0.5, \mathrm{EtOH})$; The enantiomeric excess was determined by HPLC on Chiralpak AD-3 column, hexane: isopropanol $=97: 3$; flow rate $=1.0 \mathrm{~mL} / \mathrm{min} ; \mathrm{UV}$ detection at $220 \mathrm{~nm}$; $\mathrm{t}_{\mathrm{R}}=8.9 \mathrm{~min}$ (major), $10.5 \mathrm{~min}$ (minor). ${ }^{1} \mathrm{H} \mathrm{NMR}\left(400 \mathrm{MHz}, \mathrm{CDCl}_{3}\right)$ 8: 7.30-7.26 (m, 1H), 6.96-6.86 (m, $3 \mathrm{H}), 6.80(\mathrm{dd}, J=8.2,2.2 \mathrm{~Hz}, 1 \mathrm{H}), 6.12(\mathrm{~d}, J=10.2 \mathrm{~Hz}, 1 \mathrm{H}), 3.81(\mathrm{~s}, 3 \mathrm{H}), 2.44-2.37(\mathrm{~m}, 1 \mathrm{H}), 2.34-2.23$ (m, 2H), 2.17-2.09 (m, 1H), 1.55 (s, 3H). ${ }^{13} \mathrm{C}$ NMR (101 MHz, $\left.\mathrm{CDCl}_{3}\right) \delta: 199.5,159.8,157.0,147.0$, $129.6,128.5,118.6,113.0,111.3,55.3,40.6,38.0,34.7,27.7$.<smiles>COc1ccccc1[C@]1(C)C=CC(=O)CC1</smiles>

(S)-2'-methoxy-1-methyl-2,3-dihydro-[1,1'-biphenyl]-4(1H)-one (2i): colorless oil; isolated yield: $96 \% ; 92 \%$ ee; $[\alpha]_{\mathrm{D}}^{24}=-70.8(\mathrm{c}=0.5, \mathrm{EtOH}) ;\left[\right.$ lit. $^{2}$ for the opposite enantiomer $[\alpha]_{\mathrm{D}}^{25}=+79.9(\mathrm{c}=1.0$, $\mathrm{MeOH}$ ) for 91\% ee]; The enantiomeric excess was determined by HPLC on Chiralpak AD-3 column, hexane: isopropanol $=97: 3$; flow rate $=1.0 \mathrm{~mL} / \mathrm{min} ; \mathrm{UV}$ detection at $220 \mathrm{~nm} ; \mathrm{t}_{\mathrm{R}}=8.1 \mathrm{~min}$ (major), 8.6

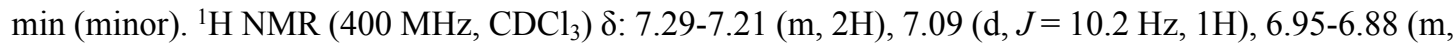
2H), $6.00(\mathrm{~d}, J=10.2 \mathrm{~Hz}, 1 \mathrm{H}), 3.82(\mathrm{~s}, 3 \mathrm{H}), 2.81-2.72(\mathrm{~m}, 1 \mathrm{H}), 2.49-2.42(\mathrm{~m}, 1 \mathrm{H}), 2.35-2.27(\mathrm{~m}, 1 \mathrm{H})$, 1.99-1.92 (m, 1H), $1.60(\mathrm{~s}, 3 \mathrm{H}) .{ }^{13} \mathrm{C} \mathrm{NMR}\left(101 \mathrm{MHz}, \mathrm{CDCl}_{3}\right) \delta: 200.3,159.8,158.1,132.9,128.6,128.0$, $126.9,120.7,112.1,55.3,40.3,35.4,34.4,26.2$<smiles>COc1ccc([C@]2(C)C=CC(=O)CC2)cc1OC</smiles>

(S)-3',4'-dimethoxy-1-methyl-2,3-dihydro-[1,1'-biphenyl]-4(1H)-one (2j): yellow oil; isolated yield: 
$96 \% ; 92 \%$ ee; $[\alpha]_{\mathrm{D}}{ }^{24}=-80.2(\mathrm{c}=0.5, \mathrm{EtOH}) ;$ The enantiomeric excess was determined by HPLC on Chiralpak AD-3 column, hexane: isopropanol = 93:7; flow rate $=1.0 \mathrm{~mL} / \mathrm{min}$; UV detection at $220 \mathrm{~nm}$; $\mathrm{t}_{\mathrm{R}}=12.1 \mathrm{~min}$ (major), $16.2 \mathrm{~min}$ (minor). ${ }^{1} \mathrm{H} \mathrm{NMR}\left(400 \mathrm{MHz}, \mathrm{CDCl}_{3}\right) \delta: 6.91(\mathrm{~d}, J=10.1 \mathrm{~Hz}, 1 \mathrm{H}), 6.89$ $6.82(\mathrm{~m}, 3 \mathrm{H}), 6.12(\mathrm{~d}, J=10.2 \mathrm{~Hz}, 1 \mathrm{H}), 3.88(\mathrm{~s}, 3 \mathrm{H}), 3.87(\mathrm{~s}, 3 \mathrm{H}), 2.45-2.38(\mathrm{~m}, 1 \mathrm{H}), 2.36-2.28(\mathrm{~m}, 1 \mathrm{H})$, 2.27-2.21 (m, 1H), 2.17-2.09 (m, 1H), $1.55(\mathrm{~s}, 3 \mathrm{H}) .{ }^{13} \mathrm{C}$ NMR (101 MHz, $\left.\mathrm{CDCl}_{3}\right) \delta: 199.7,157.4,149.2$, 148.1, 138.0, 128.6, 118.7, 111.2, 109.9, 56.2, 56.1, 40.5, 38.4, 34.7, 27.8. HRMS calculated [M+H] ${ }^{+}$ for $\mathrm{C}_{15} \mathrm{H}_{18} \mathrm{O}_{3}=247.1329$, found: 247.1327 .<smiles>C[C@]1(c2ccc3ccccc3c2)C=CC(=O)CC1</smiles>

(S)-4-methyl-4-(naphthalen-2-yl)cyclohex-2-en-1-one (2k): yellow solid; isolated yield: 98\%; 96\% ee; $[\alpha]_{\mathrm{D}}{ }^{24}=-210.2(\mathrm{c}=0.5, \mathrm{EtOH}) ;\left[\right.$ lit. $^{3}$ for the opposite enantiomer $[\alpha]_{\mathrm{D}}{ }^{25}=+245.8(\mathrm{c}=0.99, \mathrm{EtOH})$ for 97\% ee]; The enantiomeric excess was determined by HPLC on Chiralpak AD-3 column, hexane: isopropanol $=97: 3 ;$ flow rate $=1.0 \mathrm{~mL} / \mathrm{min} ; \mathrm{UV}$ detection at $210 \mathrm{~nm} ; \mathrm{t}_{\mathrm{R}}=8.6 \mathrm{~min}$ (major), $11.1 \mathrm{~min}$ (minor). ${ }^{1} \mathrm{H}$ NMR (400 MHz, $\mathrm{CDCl}_{3}$ ) $\delta: ~ 7.87-7.77(\mathrm{~m}, 3 \mathrm{H}), 7.71(\mathrm{~d}, J=1.6 \mathrm{~Hz}, 1 \mathrm{H}), 7.52-7.44(\mathrm{~m}, 3 \mathrm{H})$, $7.01(\mathrm{dd}, J=10.2,0.7 \mathrm{~Hz}, 1 \mathrm{H}), 6.19(\mathrm{~d}, J=10.2 \mathrm{~Hz}, 1 \mathrm{H}), 2.45-2.26(\mathrm{~m}, 3 \mathrm{H}), 2.22-2.14(\mathrm{~m}, 1 \mathrm{H}), 1.63$ (s, 3H). ${ }^{13} \mathrm{C}$ NMR (101 MHz, $\left.\mathrm{CDCl}_{3}\right)$ $\delta: 199.7,157.2,142.7,133.3,132.4,129.0,128.8,128.2,127.7$, $126.6,126.2,125.2,124.5,41.0,38.0,34.9,27.9$.<smiles>C[C@]1(C2CCCCC2)C=CC(=O)CC1</smiles>

(R)-1-methyl-[1,1'-bi(cyclohexan)]-2-en-4-one (2I): colorless oil; isolated yield: 95\%; 99\% ee; $[\alpha]_{\mathrm{D}}{ }^{24}$ $=-17.4(\mathrm{c}=0.5, \mathrm{EtOH}) ;\left[\right.$ lit. $^{2}$ for the opposite enantiomer $[\alpha]_{\mathrm{D}}{ }^{25}=+31.9(\mathrm{c}=1.0, \mathrm{MeOH})$ for $94 \%$ ee $]$; The enantiomeric excess was determined by HPLC on Chiralpak AD-3 column, hexane: isopropanol = 98:2; flow rate $=1.0 \mathrm{~mL} / \mathrm{min}$; UV detection at $210 \mathrm{~nm} ; \mathrm{t}_{\mathrm{R}}=6.4 \mathrm{~min}$ (minor), $6.7 \mathrm{~min}$ (major). ${ }^{1} \mathrm{H} \mathrm{NMR}$ $\left(400 \mathrm{MHz}, \mathrm{CDCl}_{3}\right) \delta: 6.73(\mathrm{dd}, J=10.3,1.3 \mathrm{~Hz}, 1 \mathrm{H}), 5.88(\mathrm{~d}, J=10.3 \mathrm{~Hz}, 1 \mathrm{H}), 2.51-2.39(\mathrm{~m}, 2 \mathrm{H}), 2.09-$ 
$2.01(\mathrm{~m}, 1 \mathrm{H}), 1.85-1.75(\mathrm{~m}, 3 \mathrm{H}), 1.72-1.62(\mathrm{~m}, 3 \mathrm{H}), 1.40-1.32(\mathrm{~m}, 1 \mathrm{H}), 1.30-1.13(\mathrm{~m}, 3 \mathrm{H}), 1.11(\mathrm{~s}, 3 \mathrm{H})$, 1.10-0.93 (m, 2H). ${ }^{13} \mathrm{C}$ NMR (101 MHz, $\left.\mathrm{CDCl}_{3}\right) \delta: 200.1,160.4,127.4,47.1,38.5,34.3,30.4,28.2,27.3$, $27.1,27.1,26.7,22.4$<smiles>CC[C@]1(C2CCCCC2)C=CC(=O)CC1</smiles>

1-ethyl-[1,1'-bi(cyclohexan)]-2-en-4-one (2m): colorless oil; isolated yield: $92 \% ; 92 \%$ ee; $[\alpha]_{\mathrm{D}}{ }^{30}=$ $11.8(\mathrm{c}=0.5, \mathrm{EtOH})$; The enantiomeric excess was determined by UPLC on Chiralpak ICU column, hexane: isopropanol $=98: 2$; flow rate $=0.6 \mathrm{~mL} / \mathrm{min}$; $\mathrm{UV}$ detection at $230 \mathrm{~nm} ; \mathrm{t}_{\mathrm{R}}=8.2$ min (major), 8.6 min (minor). ${ }^{1} \mathrm{H}$ NMR (400 MHz, $\left.\mathrm{CDCl}_{3}\right) \delta: 6.76(\mathrm{dd}, J=10.4,1.0 \mathrm{~Hz}, 1 \mathrm{H}), 5.95(\mathrm{~d}, J=10.4 \mathrm{~Hz}, 1 \mathrm{H})$, 2.52-2.37 (m, 2H), 2.05-1.97 (m, 1H), 1.82-1.48 (m, 9H), 1.29-0.99 (m, 5H), $0.88(\mathrm{t}, J=7.5 \mathrm{~Hz}, 3 \mathrm{H})$. ${ }^{13} \mathrm{C}$ NMR (101 MHz, $\left.\mathrm{CDCl}_{3}\right) \delta: 200.2,159.9,128.6,43.7,41.2,34.2,28.2,28.1,27.7,27.23,27.17,27.0$, 26.8, 8.7. HRMS calculated $[\mathrm{M}+\mathrm{H}]^{+}$for $\mathrm{C}_{14} \mathrm{H}_{22} \mathrm{O}=207.1743$, found: 207.1743 .<smiles>O=C1C=C[C@]2(CC1)CCc1ccccc12</smiles>

(S)-2',3'-dihydrospiro[cyclohexane-1,1'-inden]-2-en-4-one (4a): yellow oil; isolated yield: 96\%; 99\% ee; $[\alpha]_{\mathrm{D}}{ }^{24}=-115.6(\mathrm{c}=1.0, \mathrm{EtOH}) ;\left[\right.$ lit. $^{2}$ for the opposite enantiomer $[\alpha]_{\mathrm{D}}{ }^{25}=+111.0(\mathrm{c}=1.8, \mathrm{MeOH})$ for $86 \%$ ee]; The enantiomeric excess was determined by HPLC on Chiralpak OD-3 column, hexane: isopropanol $=97: 3$; flow rate $=1.0 \mathrm{~mL} / \mathrm{min}$; $\mathrm{UV}$ detection at $220 \mathrm{~nm}$; $\mathrm{t}_{\mathrm{R}}=8.7 \mathrm{~min}$ (major), $9.4 \mathrm{~min}$ (minor). ${ }^{1} \mathrm{H}$ NMR (400 MHz, $\left.\mathrm{CDCl}_{3}\right) \delta: 7.30-7.18(\mathrm{~m}, 3 \mathrm{H}), 7.14-7.08(\mathrm{~m}, 1 \mathrm{H}), 6.79(\mathrm{~d}, J=10.1 \mathrm{~Hz}, 1 \mathrm{H})$, $6.08(\mathrm{~d}, J=10.1 \mathrm{~Hz}, 1 \mathrm{H}), 3.12-2.95(\mathrm{~m}, 2 \mathrm{H}), 2.57-2.51(\mathrm{~m}, 2 \mathrm{H}), 2.32-2.27(\mathrm{~m}, 1 \mathrm{H}), 2.21-2.08(\mathrm{~m}, 3 \mathrm{H})$. ${ }^{13} \mathrm{C}$ NMR (101 MHz, $\mathrm{CDCl}_{3}$ ) $\delta: 199.9,156.7,147.5,143.4,128.6,127.9,127.0,125.4,123.8,49.9,38.0$, $35.2,34.0,30.7$. 


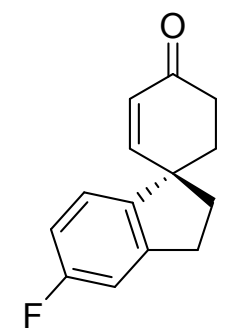

(S)-5'-fluoro-2',3'-dihydrospiro[cyclohexane-1,1'-inden]-2-en-4-one (4b): yellow oil; isolated yield: $95 \% ; 98 \%$ ee; $[\alpha]_{\mathrm{D}}{ }^{24}=-161.8(\mathrm{c}=1.0, \mathrm{EtOH}) ;\left[\right.$ lit. $^{4}$ for the opposite enantiomer $[\alpha]_{\mathrm{D}}{ }^{24}=+75.3(\mathrm{c}=1.22$, $\mathrm{CHCl}_{3}$ ) for $93 \%$ ee]; The enantiomeric excess was determined by HPLC on Chiralpak OJ-3 column, hexane: isopropanol $=97: 3$; flow rate $=1.0 \mathrm{~mL} / \mathrm{min}$; $U V$ detection at $220 \mathrm{~nm} ; \mathrm{t}_{\mathrm{R}}=14.9$ min (major), $16.9 \mathrm{~min}$ (minor). ${ }^{1} \mathrm{H} \mathrm{NMR}\left(400 \mathrm{MHz}, \mathrm{CDCl}_{3}\right) \delta: 7.03(\mathrm{dd}, J=8.3,5.2 \mathrm{~Hz}, 1 \mathrm{H}), 6.99-6.94(\mathrm{~m}, 1 \mathrm{H}), 6.89$ $(\mathrm{td}, J=8.9,2.4 \mathrm{~Hz}, 1 \mathrm{H}), 6.76(\mathrm{~d}, J=10.1 \mathrm{~Hz}, 1 \mathrm{H}), 6.07(\mathrm{~d}, J=10.1 \mathrm{~Hz}, 1 \mathrm{H}), 3.10-2.94(\mathrm{~m}, 2 \mathrm{H}), 2.54-$ $2.50(\mathrm{~m}, 2 \mathrm{H}), 2.35-2.29(\mathrm{~m}, 1 \mathrm{H}), 2.25-2.18(\mathrm{~m}, 1 \mathrm{H}), 2.15-2.06(\mathrm{~m}, 2 \mathrm{H}) .{ }^{13} \mathrm{C} \mathrm{NMR}\left(101 \mathrm{MHz}, \mathrm{CDCl}_{3}\right) \delta$ : 199.7, $163.0(\mathrm{~d}, J=244.9 \mathrm{~Hz}), 156.3,145.7$ (d, $J=8.1 \mathrm{~Hz}), 143.1,128.9,124.8(\mathrm{~d}, J=9.1 \mathrm{~Hz}), 113.9$ $(\mathrm{d}, J=22.8 \mathrm{~Hz}), 112.4(\mathrm{~d}, J=22.0 \mathrm{~Hz}), 49.2,38.3,35.1,34.2,30.7 .{ }^{19} \mathrm{~F} \mathrm{NMR}\left(376 \mathrm{MHz}, \mathrm{CDCl}_{3}\right) \delta-$ 115.89<smiles>O=C1C=C[C@]2(CC1)CCc1cc(Cl)ccc12</smiles>

(S)-5'-chloro-2',3'-dihydrospiro[cyclohexane-1,1'-inden]-2-en-4-one (4c): yellow solid; isolated yield: $95 \%$; $99 \%$ ee; $[\alpha]_{\mathrm{D}}{ }^{30}=-164.8(\mathrm{c}=0.5, \mathrm{EtOH}) ;\left[\right.$ lit. ${ }^{4}$ for the opposite enantiomer $[\alpha]_{\mathrm{D}}{ }^{24}=+68.2(\mathrm{c}$ $=1.13, \mathrm{CHCl}_{3}$ ) for $90 \%$ ee]; The enantiomeric excess was determined by HPLC on Chiralpak OD-3 column, hexane: isopropanol $=97: 3$; flow rate $=1.0 \mathrm{~mL} / \mathrm{min} ; \mathrm{UV}$ detection at $220 \mathrm{~nm} ; \mathrm{t}_{\mathrm{R}}=8.5 \mathrm{~min}$ (minor), $9.0 \mathrm{~min}$ (major). ${ }^{1} \mathrm{H}$ NMR (400 MHz, $\left.\mathrm{CDCl}_{3}\right) \delta: 7.26(\mathrm{~s}, 1 \mathrm{H}), 7.18(\mathrm{~d}, J=8.1 \mathrm{~Hz}, 1 \mathrm{H}), 7.02(\mathrm{~d}$, $J=8.1 \mathrm{~Hz}, 1 \mathrm{H}), 6.74(\mathrm{~d}, J=10.1 \mathrm{~Hz}, 1 \mathrm{H}), 6.08(\mathrm{~d}, J=10.1 \mathrm{~Hz}, 1 \mathrm{H}), 3.10-2.93(\mathrm{~m}, 2 \mathrm{H}), 2.54-2.48(\mathrm{~m}$, 2H), 2.34-2.28 (m, 1H), 2.22-2.16 (m, 1H), 2.12-2.04 (m, 2H). ${ }^{13} \mathrm{C}$ NMR (101 MHz, $\left.\mathrm{CDCl}_{3}\right) \delta: 199.5$, $155.9,146.1,145.3,133.7,129.0,127.2,125.6,124.8,49.4,38.0,35.0,34.0,30.5$. 
<smiles>O=C1C=C[C@@]2(CC1)CCc1ccc(Br)cc12</smiles>

(S)-6'-bromo-2',3'-dihydrospiro[cyclohexane-1,1'-inden]-2-en-4-one (4d): white solid; isolated yield: $97 \% ; 99 \%$ ee; $[\alpha]_{\mathrm{D}}{ }^{24}=-183.0(\mathrm{c}=0.5, \mathrm{EtOH})$; The enantiomeric excess was determined by UPLC on Chiralpak IAU column, hexane: isopropanol $=99: 1 ;$ flow rate $=0.5 \mathrm{~mL} / \mathrm{min} ; \mathrm{UV}$ detection at $210 \mathrm{~nm}$; $\mathrm{t}_{\mathrm{R}}=5.5 \mathrm{~min}$ (major), $5.9 \mathrm{~min}$ (minor). ${ }^{1} \mathrm{H} \mathrm{NMR}\left(400 \mathrm{MHz}, \mathrm{CDCl}_{3}\right) \delta: 7.35(\mathrm{dd}, J=8.0,1.8 \mathrm{~Hz}, 1 \mathrm{H}), 7.22$ $(\mathrm{d}, J=1.6 \mathrm{~Hz}, 1 \mathrm{H}), 7.15(\mathrm{~d}, J=8.0 \mathrm{~Hz}, 1 \mathrm{H}), 6.75(\mathrm{~d}, J=10.1 \mathrm{~Hz}, 1 \mathrm{H}), 6.09(\mathrm{~d}, J=10.1 \mathrm{~Hz}, 1 \mathrm{H}), 3.05-$ $2.90(\mathrm{~m}, 2 \mathrm{H}), 2.53(\mathrm{t}, J=6.8 \mathrm{~Hz}, 2 \mathrm{H}), 2.33-2.27(\mathrm{~m}, 1 \mathrm{H}), 2.20-2.05(\mathrm{~m}, 3 \mathrm{H}) .{ }^{13} \mathrm{C}$ NMR $(101 \mathrm{MHz}$, $\left.\mathrm{CDCl}_{3}\right) \delta: 199.4,155.6,150.1,142.3,131.0,129.2,127.1,126.9,120.7,50.0,38.0,35.1,34.0,30.3$.<smiles>COc1cccc2c1CC[C@@]21C=CC(=O)CC1</smiles>

(S)-4'-methoxy-2',3'-dihydrospiro[cyclohexane-1,1'-inden]-2-en-4-one (4e): colorless oil; isolated yield: $94 \% ; 99 \%$ ee; $[\alpha]_{\mathrm{D}}^{24}=-161.4(\mathrm{c}=0.5, \mathrm{EtOH})$; The enantiomeric excess was determined by HPLC on Chiralpak OJ-3 column, hexane: isopropanol $=97: 3$; flow rate $=1.0 \mathrm{~mL} / \mathrm{min}$; UV detection at 220 $\mathrm{nm} ; \mathrm{t}_{\mathrm{R}}=16.9 \mathrm{~min}$ (major), $19.6 \mathrm{~min}$ (minor). ${ }^{1} \mathrm{H} \mathrm{NMR}\left(400 \mathrm{MHz}, \mathrm{CDCl}_{3}\right) \delta: 7.20(\mathrm{t}, J=7.8 \mathrm{~Hz}, 1 \mathrm{H})$, $6.81-6.71(\mathrm{~m}, 3 \mathrm{H}), 6.06(\mathrm{~d}, J=10.1 \mathrm{~Hz}, 1 \mathrm{H}), 3.85(\mathrm{~s}, 3 \mathrm{H}), 3.04-2.89(\mathrm{~m}, 2 \mathrm{H}), 2.57-2.48(\mathrm{~m}, 2 \mathrm{H}), 2.33-$ $2.27(\mathrm{~m}, 1 \mathrm{H}), 2.18-2.08(\mathrm{~m}, 3 \mathrm{H}) .{ }^{13} \mathrm{C} \mathrm{NMR}\left(101 \mathrm{MHz}, \mathrm{CDCl}_{3}\right) \delta: 199.9,156.6,156.5,149.4,130.8,128.5$ $116.0,109.4,55.5,50.3,37.7,35.2,34.1,27.3$.<smiles>COc1cc2c(c(OC)c1)[C@]1(C=CC(=O)CC1)CC2</smiles> 
isolated yield: $96 \% ; 99 \%$ ee; $[\alpha]_{\mathrm{D}}{ }^{30}=-164.5(\mathrm{c}=0.5, \mathrm{EtOH}) ;\left[\mathrm{lit}^{5}[\alpha]_{\mathrm{D}}{ }^{22}=\right.$

$-175.0(\mathrm{c}=0.22, \mathrm{EtOH})]$; The enantiomeric excess was determined by HPLC on Chiralpak AD-3 column, hexane: isopropanol $=97: 3$; flow rate $=1.0 \mathrm{~mL} / \mathrm{min}$; $\mathrm{UV}$ detection at $210 \mathrm{~nm} ; \mathrm{t}_{\mathrm{R}}=14.0 \mathrm{~min}$ (minor), $15.2 \mathrm{~min}$ (major). ${ }^{1} \mathrm{H}$ NMR $\left(400 \mathrm{MHz}, \mathrm{CDCl}_{3}\right) \delta: 6.88(\mathrm{dd}, J=10.1,1.6 \mathrm{~Hz}, 1 \mathrm{H}), 6.42-6.37(\mathrm{~m}, 1 \mathrm{H})$, $6.29(\mathrm{~d}, J=1.7 \mathrm{~Hz}, 1 \mathrm{H}), 5.95(\mathrm{~d}, J=10.1 \mathrm{~Hz}, 1 \mathrm{H}), 3.80(\mathrm{~s}, 3 \mathrm{H}), 3.72(\mathrm{~s}, 3 \mathrm{H}), 3.04-2.88(\mathrm{~m}, 2 \mathrm{H}), 2.62-$ 2.50(m, 2H), 2.48-2.39 (m, 1H), 2.35-2.29 (m, 1H), 2.06-2.01 (m, 1H), 1.95-1.89 (m, 1H). ${ }^{13} \mathrm{C}$ NMR $\left(101 \mathrm{MHz}, \mathrm{CDCl}_{3}\right)$ ): 200.3, 161.5, 158.9, 157.2, 146.2, 127.8, 126.7, 101.1, 97.4, 55.8, 55.3, 48.7, 35.8, $35.5,31.6,31.2$.<smiles>O=C1C=C[C@]2(CCCc3ccccc32)CC1</smiles>

(S)-3',4'-dihydro-2'H-spiro[cyclohexane-1,1'-naphthalen]-2-en-4-one (4g): white solid; isolated yield: $95 \% ; 98 \%$ ee; $[\alpha]_{D}{ }^{24}=-74.1(\mathrm{c}=0.5, \mathrm{EtOH}) ;\left[\right.$ lit. $^{3}$ for the opposite enantiomer $[\alpha]_{\mathrm{D}}{ }^{27}=+69.9(\mathrm{c}=$ $1.05, \mathrm{EtOH}$ ) for $90 \%$ ee]; The enantiomeric excess was determined by HPLC on Chiralpak AD-3 column, hexane: isopropanol $=97: 3$; flow rate $=1.0 \mathrm{~mL} / \mathrm{min} ; \mathrm{UV}$ detection at $254 \mathrm{~nm} ; \mathrm{t}_{\mathrm{R}}=7.4 \mathrm{~min}$ (minor), 7.9 $\min$ (major). ${ }^{1} \mathrm{H}$ NMR $\left(400 \mathrm{MHz}, \mathrm{CDCl}_{3}\right) \delta: 7.18-7.11(\mathrm{~m}, 4 \mathrm{H}), 6.75(\mathrm{dd}, J=10.1,1.5 \mathrm{~Hz}, 1 \mathrm{H}), 6.09(\mathrm{~d}$, $J=10.1 \mathrm{~Hz}, 1 \mathrm{H}), 2.87-2.84(\mathrm{~m}, 2 \mathrm{H}), 2.59-2.43(\mathrm{~m}, 2 \mathrm{H}), 2.28-2.15(\mathrm{~m}, 2 \mathrm{H}), 2.09-2.03(\mathrm{~m}, 1 \mathrm{H}), 1.98-1.85$ (m, 2H), $1.75(\mathrm{td}, J=12.3,3.3 \mathrm{~Hz}, 1 \mathrm{H}) .{ }^{13} \mathrm{C}$ NMR $\left(101 \mathrm{MHz}, \mathrm{CDCl}_{3}\right) \delta: 199.3,158.4,141.3,136.2$, $129.5,128.4,128.0,126.7,126.2,40.1,35.7,34.1,31.8,29.5,19.0$.<smiles>O=C1C=CC2(CCCc3ccc(Cl)cc32)CC1</smiles>

(S)-7'-chloro-3',4'-dihydro-2'H-spiro[cyclohexane-1,1'-naphthalen]-2-en-4-one (4h): yellow solid; isolated yield: $98 \%$; 98\% ee; $[\alpha]_{D}{ }^{24}=-106.6(\mathrm{c}=0.5, \mathrm{EtOH}) ;\left[{ }_{1 i t}{ }^{6}[\alpha]_{\mathrm{D}}^{23}=-153.0(\mathrm{c}=0.10, \mathrm{EtOH})\right.$ for 84\% ee]; The enantiomeric excess was determined by HPLC on Chiralpak OD-3 column, hexane: isopropanol $=97: 3 ;$ flow rate $=1.0 \mathrm{~mL} / \mathrm{min} ; \mathrm{UV}$ detection at $220 \mathrm{~nm} ; \mathrm{t}_{\mathrm{R}}=7.8 \mathrm{~min}($ minor), $8.3 \mathrm{~min}$ 
(major). ${ }^{1} \mathrm{H}$ NMR (400 MHz, $\mathrm{CDCl}_{3}$ ) $8: 7.14-7.04(\mathrm{~m}, 3 \mathrm{H}), 6.70(\mathrm{dd}, J=10.1,1.6 \mathrm{~Hz}, 1 \mathrm{H}), 6.10(\mathrm{~d}, J=$ $10.3 \mathrm{~Hz}, 1 \mathrm{H}), 2.88-2.73(\mathrm{~m}, 2 \mathrm{H}), 2.59-2.41(\mathrm{~m}, 2 \mathrm{H}), 2.29-2.22(\mathrm{~m}, 1 \mathrm{H}), 2.19-2.11(\mathrm{~m}, 1 \mathrm{H}), 2.08-2.04$ (m, 1H), 1.99-1.92 (m, 1H), 1.91-1.83 (m, 1H), 1.76-1.67 (m, 1H). ${ }^{13} \mathrm{C}$ NMR (101 MHz, $\left.\mathrm{CDCl}_{3}\right)$ ): 199.2, $157.6,143.5,134.8,131.9,131.1,128.7,128.5,127.1,40.4,35.8,34.2,31.3,29.2,19.1$.<smiles>O=C1C=C[C@]2(CCCc3sccc32)CC1</smiles>

(S)-6,7-dihydro-5H-spiro[benzo[b]thiophene-4,1'-cyclohexan]-2'-en-4'-one (4i): yellow solid; isolated yield: $95 \%$; $98 \%$ ee; $[\alpha]_{\mathrm{D}}{ }^{24}=-159.2(\mathrm{c}=0.5, \mathrm{EtOH})$; The enantiomeric excess was determined by HPLC on Chiralpak OD-3 column, hexane: isopropanol $=97: 3$; flow rate $=1.0 \mathrm{~mL} / \mathrm{min}$; UV detection at $220 \mathrm{~nm} ; \mathrm{t}_{\mathrm{R}}=9.5 \mathrm{~min}$ (minor), $10.3 \mathrm{~min}$ (major). ${ }^{1} \mathrm{H} \mathrm{NMR}\left(400 \mathrm{MHz}, \mathrm{CDCl}_{3}\right)$ 8: $7.08(\mathrm{~d}, J=5.2 \mathrm{~Hz}$, 1H), 6.76 (d, $J=5.2 \mathrm{~Hz}, 1 \mathrm{H}), 6.70(\mathrm{dd}, J=10.1,1.2 \mathrm{~Hz}, 1 \mathrm{H}), 6.01$ (d, $J=10.1 \mathrm{~Hz}, 1 \mathrm{H}), 2.92-2.75(\mathrm{~m}$, 2H), 2.60-2.48 (m, 2H), 2.27-2.20 (m, 1H), 2.18-2.10 (m, 1H), $2.00(\mathrm{dt}, J=11.2,4.7 \mathrm{~Hz}, 2 \mathrm{H}), 1.96-1.86$ (m, 1H), 1.78-1.73 (m, 1H). ${ }^{13} \mathrm{C}$ NMR (101 MHz, $\left.\mathrm{CDCl}_{3}\right) \delta: 199.7,157.9,139.4,136.8,127.9,126.5$, 122.7, 38.9, 34.7, 34.5, 32.4, 25.2, 20.2. HRMS calculated $[\mathrm{M}+\mathrm{H}]^{+}$for $\mathrm{C}_{13} \mathrm{H}_{14} \mathrm{OS}=219.0838$, found: 219.0837.<smiles>O=C1C=CC2(CCOc3ccccc32)CC1</smiles>

(R)-spiro[chromane-4,1'-cyclohexan]-2'-en-4'-one (4j): white solid; isolated yield: $96 \%$; $99 \%$ ee; $[\alpha]_{\mathrm{D}}{ }^{24}=-86.2(\mathrm{c}=0.5, \mathrm{EtOH}) ;\left[\mathrm{lit}^{6}[\alpha]_{\mathrm{D}}{ }^{26}=-97.4(\mathrm{c}=0.6, \mathrm{EtOH})\right.$ for $88 \%$ ee $]$; The enantiomeric excess was determined by HPLC on Chiralpak AD-3 column, hexane: isopropanol $=97: 3$; flow rate $=1.0$ $\mathrm{mL} / \mathrm{min}$; UV detection at $220 \mathrm{~nm}$; $\mathrm{t}_{\mathrm{R}}=11.2 \mathrm{~min}$ (minor), $11.8 \mathrm{~min}$ (major). ${ }^{1} \mathrm{H} \mathrm{NMR}\left(400 \mathrm{MHz}, \mathrm{CDCl}_{3}\right.$ ) $\delta: 7.18-7.14(\mathrm{~m}, 1 \mathrm{H}), 7.08(\mathrm{dd}, J=7.8,1.6 \mathrm{~Hz}, 1 \mathrm{H}), 6.93-6.84(\mathrm{~m}, 2 \mathrm{H}), 6.68(\mathrm{dd}, J=10.1,1.3 \mathrm{~Hz}, 1 \mathrm{H})$, $6.14(\mathrm{~d}, J=10.1 \mathrm{~Hz}, 1 \mathrm{H}), 4.39-4.33(\mathrm{~m}, 1 \mathrm{H}), 4.26-4.18(\mathrm{~m}, 1 \mathrm{H}), 2.57-2.48(\mathrm{~m}, 2 \mathrm{H}), 2.36-2.24(\mathrm{~m}, 2 \mathrm{H})$, 2.14-2.07 (m, 2H). ${ }^{13} \mathrm{C}$ NMR (101 MHz, $\left.\mathrm{CDCl}_{3}\right)$ 8: 199.2, 156.5, 154.0, 129.3, 129.0, 128.9, 126.8, 121.0, 
<smiles>O=C1C=C[C@@]2(CCSc3ccccc32)CC1</smiles>

$(\boldsymbol{R})$-spiro[cyclohexane-1,4'-thiochroman]-2-en-4-one (4k): white solid; isolated yield: 96\%; 98\% ee; $[\alpha]_{\mathrm{D}}{ }^{24}=-23.2(\mathrm{c}=0.5, \mathrm{EtOH}) ;\left[\mathrm{lit}^{6}{ }^{6}[\alpha]_{\mathrm{D}}{ }^{25}=-19.5(\mathrm{c}=0.8, \mathrm{EtOH})\right.$ for $82 \%$ ee $]$; The enantiomeric excess was determined by HPLC on Chiralpak AD-3 column, hexane: isopropanol $=97: 3$; flow rate $=1.0$ $\mathrm{mL} / \mathrm{min}$; UV detection at $210 \mathrm{~nm} ; \mathrm{t}_{\mathrm{R}}=11.8 \mathrm{~min}$ (minor), $12.7 \mathrm{~min}$ (major). ${ }^{1} \mathrm{H}$ NMR (400 MHz, $\mathrm{CDCl}_{3}$ ) $\delta: 7.19-7.10(\mathrm{~m}, 3 \mathrm{H}), 7.06-7.00(\mathrm{~m}, 1 \mathrm{H}), 6.75(\mathrm{~d}, J=10.1 \mathrm{~Hz}, 1 \mathrm{H}), 6.20(\mathrm{~d}, J=10.1 \mathrm{~Hz}, 1 \mathrm{H}), 3.27(\mathrm{td}, J$ $=12.9,3.6 \mathrm{~Hz}, 1 \mathrm{H}), 3.02(\mathrm{dt}, J=12.6,4.0 \mathrm{~Hz}, 1 \mathrm{H}), 2.48-2.40(\mathrm{~m}, 2 \mathrm{H}), 2.31-2.21(\mathrm{~m}, 2 \mathrm{H}), 2.17-2.10(\mathrm{~m}$, 2H). ${ }^{13} \mathrm{C} \mathrm{NMR}\left(101 \mathrm{MHz}, \mathrm{CDCl}_{3}\right) \delta: 199.5,156.0,136.0,132.5,129.8,128.9,127.8,127.4,124.4,39.3$, $33.7,33.5,32.3,22.4$.<smiles>O=C1C=CC2(CCCCc3ccccc32)CC1</smiles>

(S)-6,7,8,9-tetrahydrospiro[benzo[7] annulene-5,1'-cyclohexan]-2'-en-4'-one (4I): colorless oil; isolated yield: $97 \%$; $96 \%$ ee; $[\alpha]_{D}{ }^{24}=-23.4(\mathrm{c}=0.5, \mathrm{EtOH}) ;\left[1 \mathrm{lit} .^{6}[\alpha]_{\mathrm{D}}{ }^{25}=-17.0(\mathrm{c}=0.71, \mathrm{EtOH})\right.$ for $23 \%$ ee]; The enantiomeric excess was determined by HPLC on Chiralpak OD-3 column, hexane: isopropanol $=97: 3$; flow rate $=1.0 \mathrm{~mL} / \mathrm{min} ; \mathrm{UV}$ detection at $220 \mathrm{~nm} ; \mathrm{t}_{\mathrm{R}}=7.3 \mathrm{~min}$ (major), $7.8 \mathrm{~min}$ (minor). ${ }^{1} \mathrm{H}$ NMR (400 MHz, $\left.\mathrm{CDCl}_{3}\right)$ 8: 7.23-7.08 (m, 4H), $6.89(\mathrm{dd}, J=10.2,1.7 \mathrm{~Hz}, 1 \mathrm{H}), 6.12(\mathrm{~d}, J=10.2 \mathrm{~Hz}, 1 \mathrm{H}), 3.12-$ $3.05(\mathrm{~m}, 1 \mathrm{H}), 2.85(\mathrm{ddd}, J=15.0,6.0,2.0 \mathrm{~Hz}, 1 \mathrm{H}), 2.59-2.51(\mathrm{~m}, 1 \mathrm{H}), 2.39-2.30(\mathrm{~m}, 1 \mathrm{H}), 2.24-2.07(\mathrm{~m}$, 2H), 2.03-1.95 (m, 1H), 1.94-1.84 (m, 4H), 1.59-1.46 (m, 1H). ${ }^{13} \mathrm{C}$ NMR (101 MHz, $\left.\mathrm{CDCl}_{3}\right) \delta: 200.4$, 159.2, 142.4, 142.2, 132.3, 129.0, 127.3, 127.1, 126.3, 45.8, 39.7, 37.4, 34.6, 32.1, 28.1, 25.8. 


\section{Deuterium Labeling Studies.}

A stock solution was made by mixing $\mathrm{Ni}(\mathrm{OTf})_{2}(3.56 \mathrm{mg}, 0.01 \mathrm{mmol})$ with $(S, S)-\mathrm{Ph}-\mathrm{BPE}(5.6 \mathrm{mg}$, $0.011 \mathrm{mmol})$ in a $1: 1.1 \mathrm{molar}$ ratio in $\mathrm{MeOH} /$ toluene $(1: 1,1 \mathrm{ml})$ at room temperature for $30 \mathrm{~min}$ in a nitrogen-filled glovebox. An aliquot of the catalyst solution $(0.1 \mathrm{~mL}, 0.001 \mathrm{mmol})$ was transferred by syringe into the vials charged with substrate $1 \mathbf{a}(0.1 \mathrm{mmol})$ in anhydrous toluene $(0.9 \mathrm{~mL})$. The vial was subsequently transferred into an autoclave into which deuterium gas was charged. The reaction was then stirred under $\mathrm{D}_{2}(50 \mathrm{~atm})$ at $50{ }^{\circ} \mathrm{C}$ for $24 \mathrm{~h}$. The deuterium gas was released slowly and carefully. The crude product was purified by flash chromatography on silica gel to give the product.

م

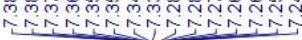

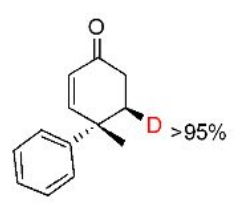

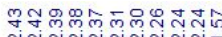

ninininginin

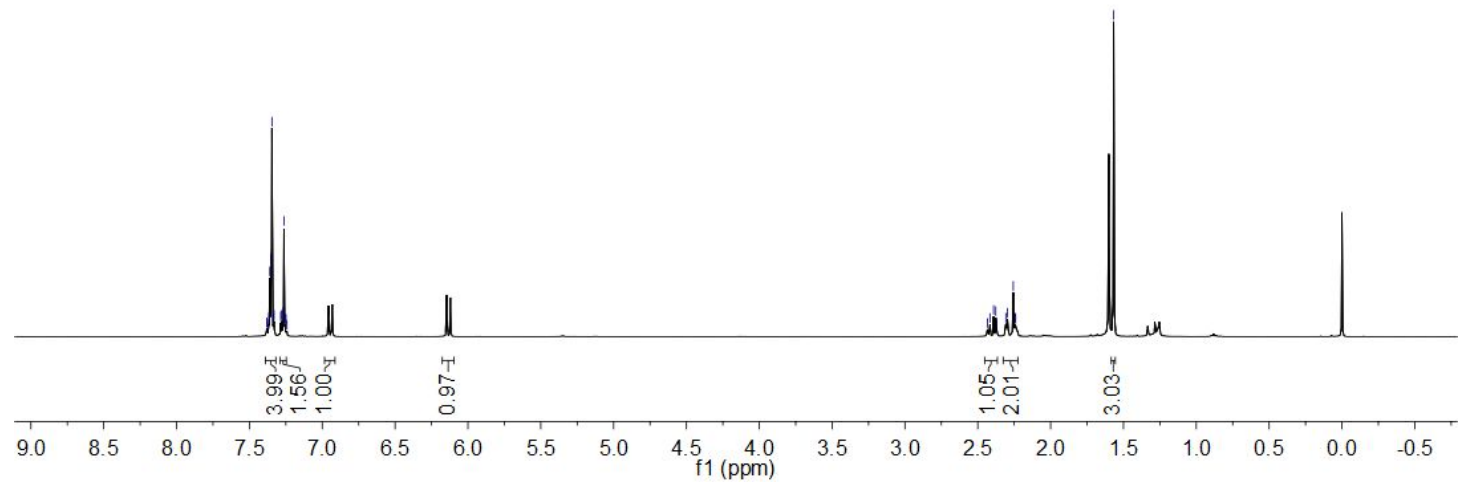




\section{References}

1. Han, Y.; Breitler, S.; Zheng, S,-L,; Corey, E. J. Enantioselective conversion of achiral cyclohexadienones to chiral cyclohexenones by desymmetrization. Org. Lett. 2016, 18, 6172-6175.

2. Bokka, A.; Mao, J. X.; Hartung, J; Martinez, S. R.; Simanis, J. A.; Nam, K.; Jeon, J.; Shen, X. Asymmetric synthesis of remote quaternary centers by copper catalyzed desymmetrization: an enantioselective total synthesis of (+)-mesembrine. Org. Lett. 2018, 20, 5158-5162.

3. Inokoishi, Y,; Sasakura, N,; Nakano, K.; Ichikawa, Y.; Kotsuki, H. A new powerful strategy for the organocatalytic asymmetric construction of a quaternary carbon stereogenic center. Org. Lett. 2010, $12,1616-1619$.

4. Zhu, L.; Zhang, L.; Luo S. Catalytic desymmetrizing dehydrogenation of 4-substituted cyclohexanones through enamine oxidation. Angew. Chem. Int. Ed. 2018, 57, 2253-2258.

5. Yoshida, K.; Itatsu, Y.; Fujino, Y.; Inoue, H.; Takao, K. Enantioselective organocatalytic construction of spiroindane derivatives by intramolecular Friedel-Crafts-type 1,4-addition. Angew. Chem. Int. Ed. 2016, 55, 6734-6738

6. Naganawa, Y.; Kawagishi, M.; Ito, J.; Nishiyama H. Asymmetric induction at remote quaternary centers of cyclohexadienones by rhodium-catalyzed conjugate hydrosilylation. Angew. Chem. Int. Ed. 2016, 55, 6873-6876. 


\section{NMR spectra}

$1 \mathrm{e}$

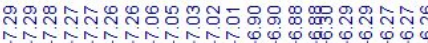<smiles>CC1(c2ccc(F)cc2)C=CC(=O)C=C1</smiles>

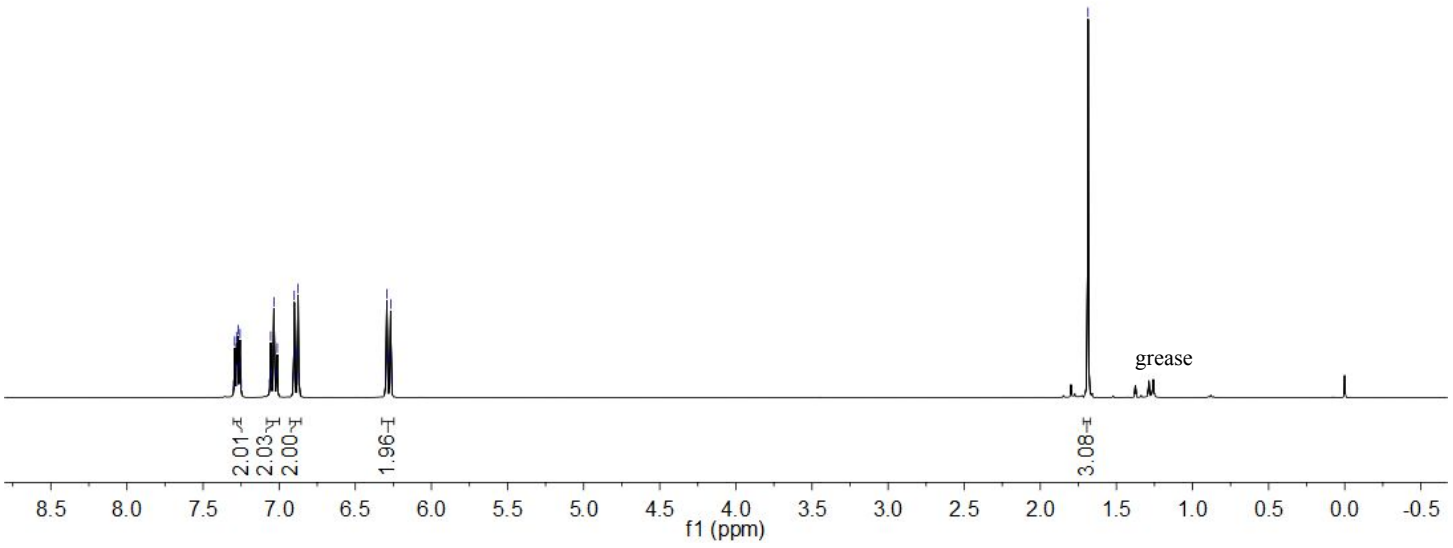
के
1

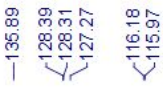

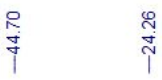<smiles>CC1(c2ccc(F)cc2)C=CC(=O)C=C1</smiles>

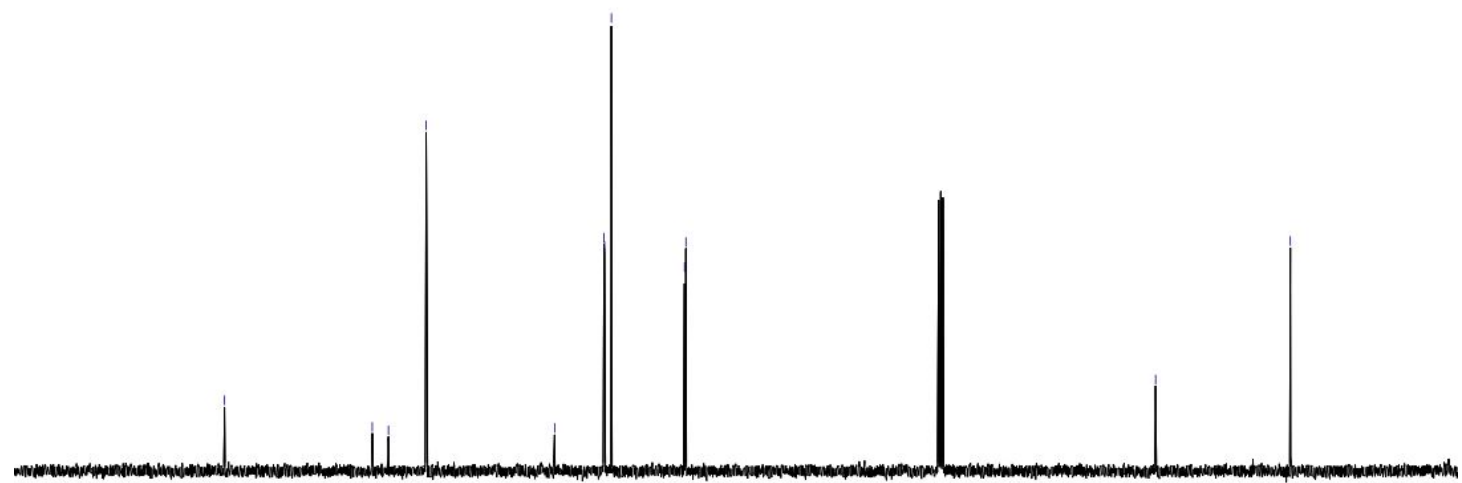

$\begin{array}{llllllllllllllllllllll}210 & 200 & 190 & 180 & 170 & 160 & 150 & 140 & 130 & 120 & 110 & 100 & 90 & 80 & 70 & 60 & 50 & 40 & 30 & 20 & 10 & 0\end{array}$ 
lxx-le. 1. fid

迎

$-6000000$

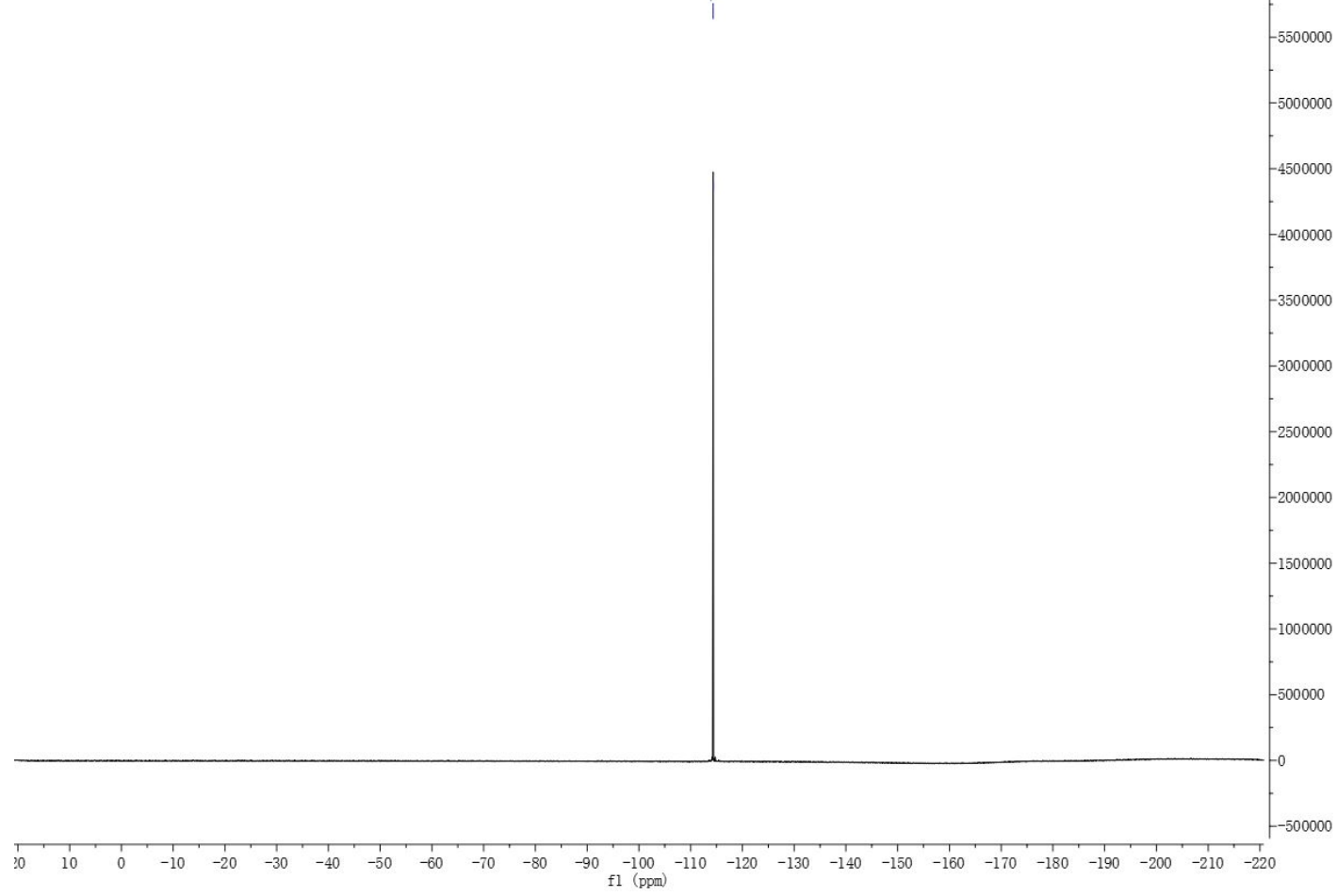


$1 \mathrm{~g}$

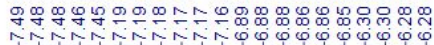

-<smiles>CC1(c2ccc(Br)cc2)C=CC(=O)C=C1</smiles>

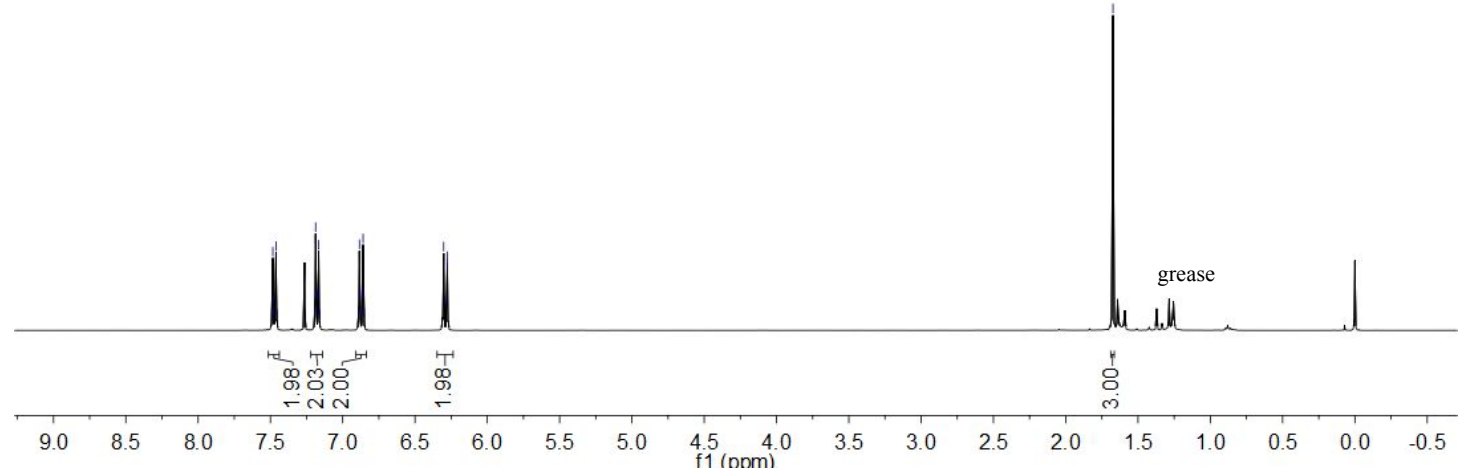<smiles>CC1(c2ccc(Br)cc2)C=CC(=O)C=C1</smiles>

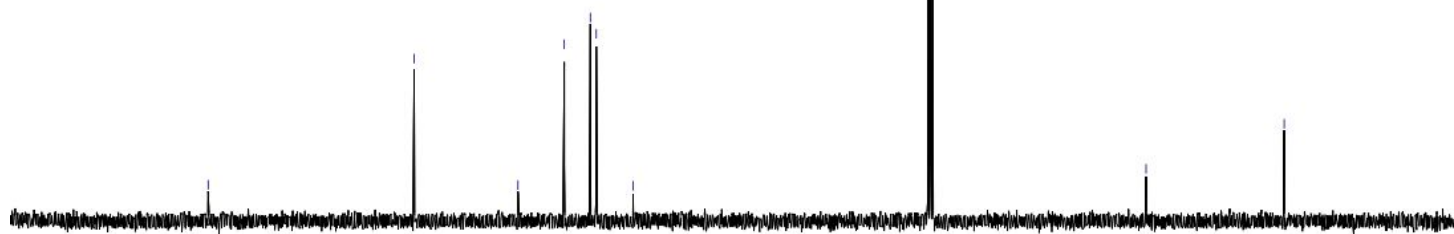

$\begin{array}{llllllllllllllllllllll}210 & 200 & 190 & 180 & 170 & 160 & 150 & 140 & 130 & 120 & 110 & 100 & 90 & 80 & 70 & 60 & 50 & 40 & 30 & 20 & 10 & 0\end{array}$ 


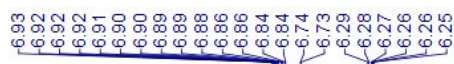

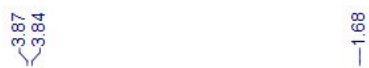

MeO

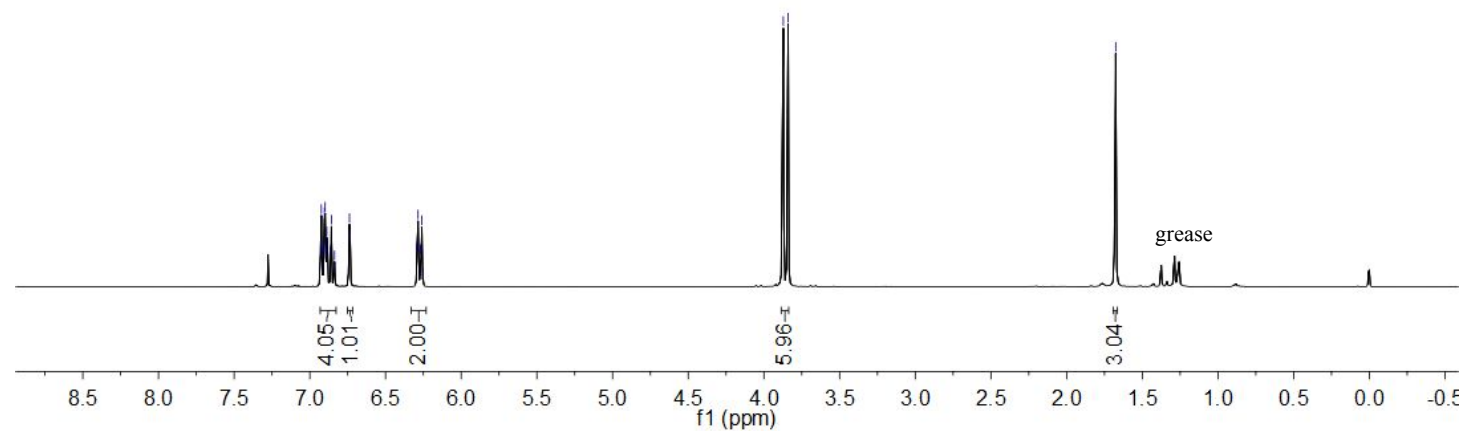

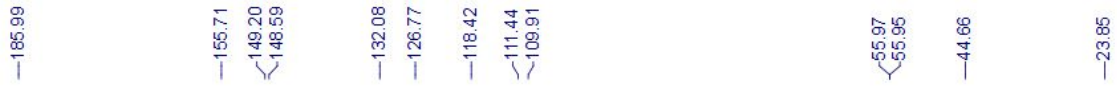<smiles>COc1ccc(C2(C)C=CC(=O)C=C2)cc1OC</smiles>

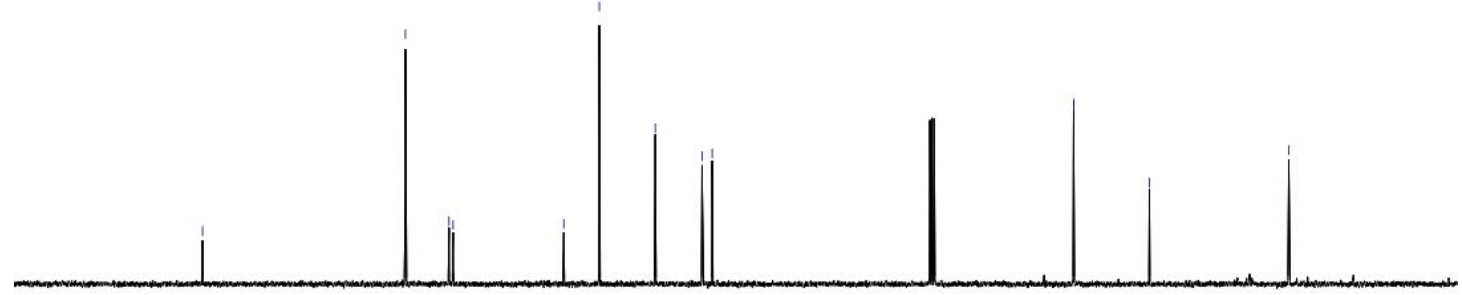

$\begin{array}{llllllllllllllllllllll}210 & 200 & 190 & 180 & 170 & 160 & 150 & 140 & 130 & 120 & \begin{array}{c}110 \\ \mathrm{f} 1(\mathrm{ppm})\end{array} & \begin{array}{l}100 \\ 90\end{array} & 80 & 70 & 60 & 50 & 40 & 30 & 20 & 10 & 0\end{array}$ 
<smiles>CCC1(C2CCCCC2)C=CC(=O)C=C1</smiles>

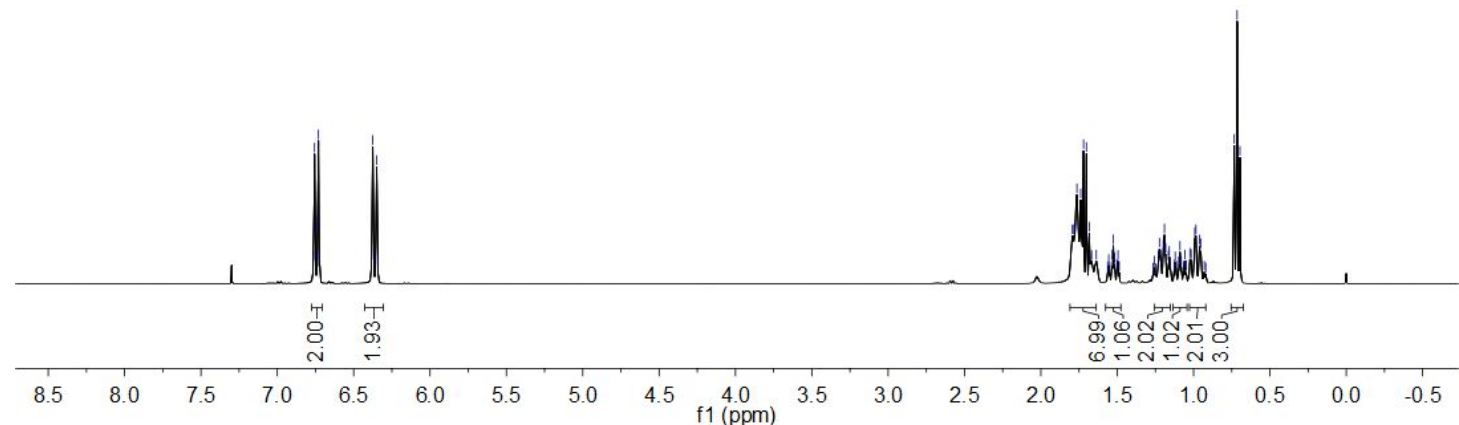<smiles>CCC1(C2CCCCC2)C=CC(=O)C=C1</smiles>

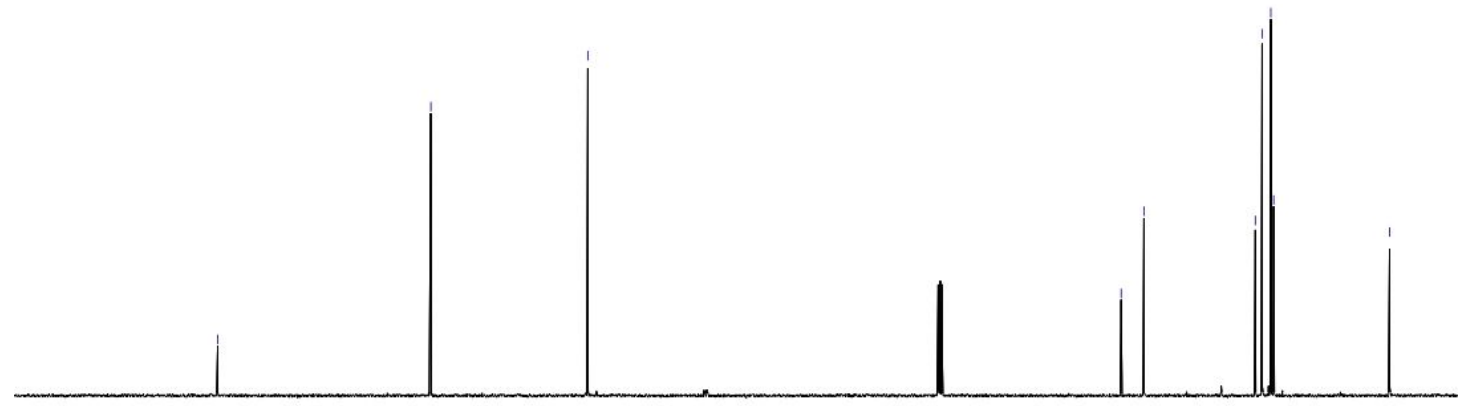

$\begin{array}{llllllllllllllllllllll}210 & 200 & 190 & 180 & 170 & 160 & 150 & 140 & 130 & 120 & \begin{array}{c}110 \\ \mathrm{f} 1(\mathrm{ppm})\end{array} & 100 & 90 & 80 & 70 & 60 & 50 & 40 & 30 & 20 & 10 & 0\end{array}$ 
3i

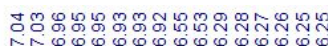

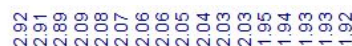

(1)

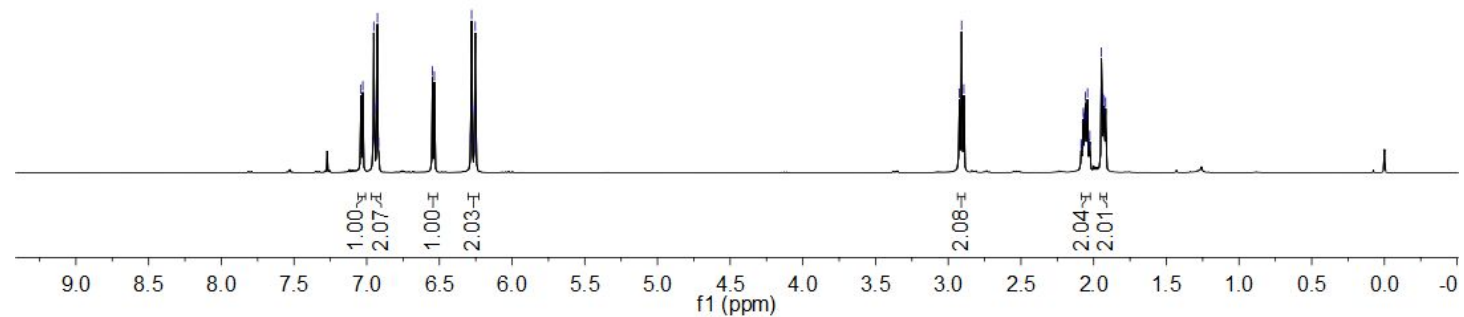<smiles>O=C1C=CC2(C=C1)CCCc1sccc12</smiles>

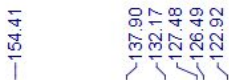

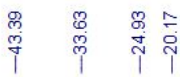

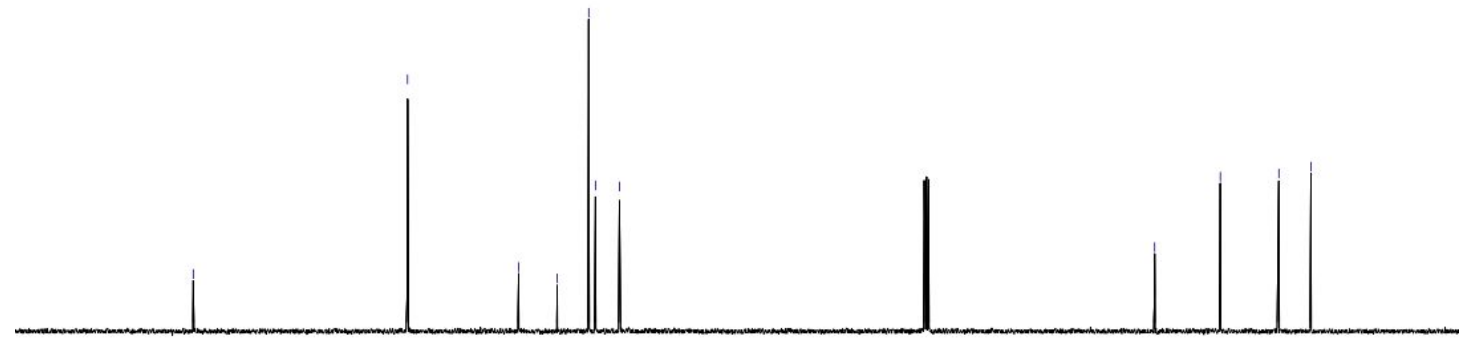

$\begin{array}{llllllllllllllllllllll}210 & 200 & 190 & 180 & 170 & 160 & 150 & 140 & 130 & 120 & \begin{array}{c}110 \\ \mathrm{f} 1(\mathrm{ppm})\end{array} & 90 & 80 & 70 & 60 & 50 & 40 & 30 & 20 & 10 & 0\end{array}$ 


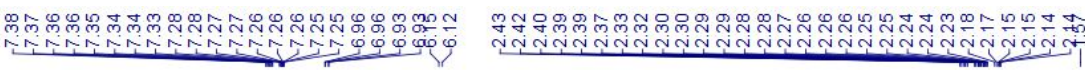<smiles>C[C@]1(c2ccccc2)C=CC(=O)CC1</smiles>

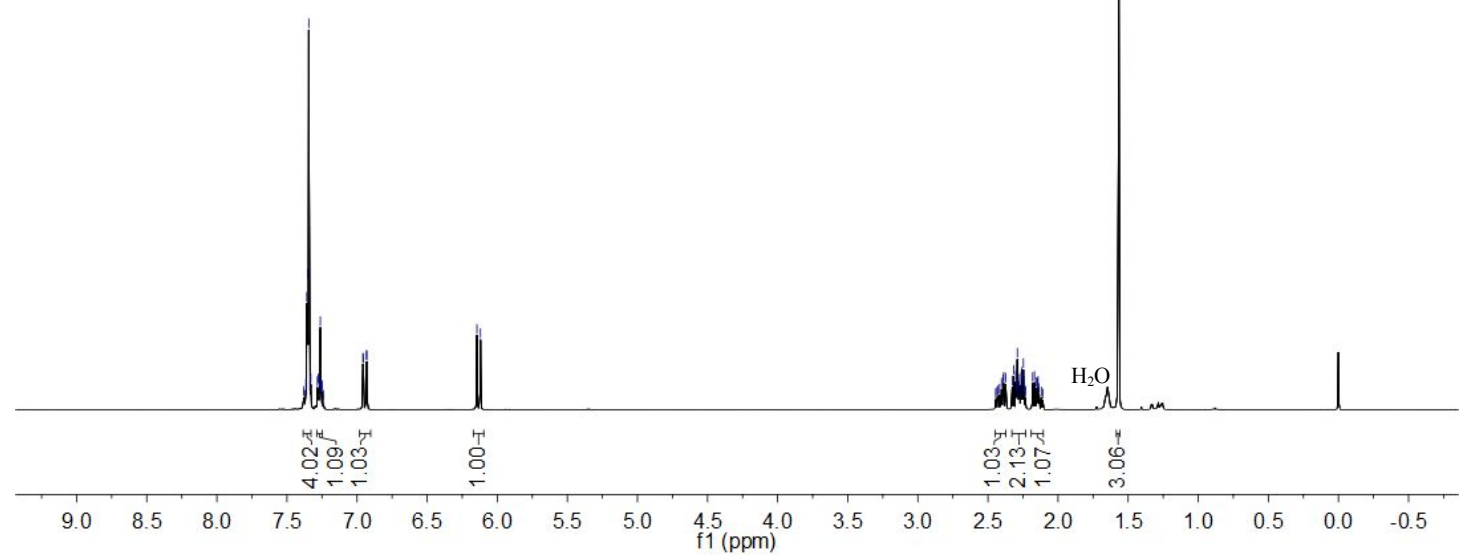<smiles>C[C@]1(c2ccccc2)C=CC(=O)CC1</smiles>
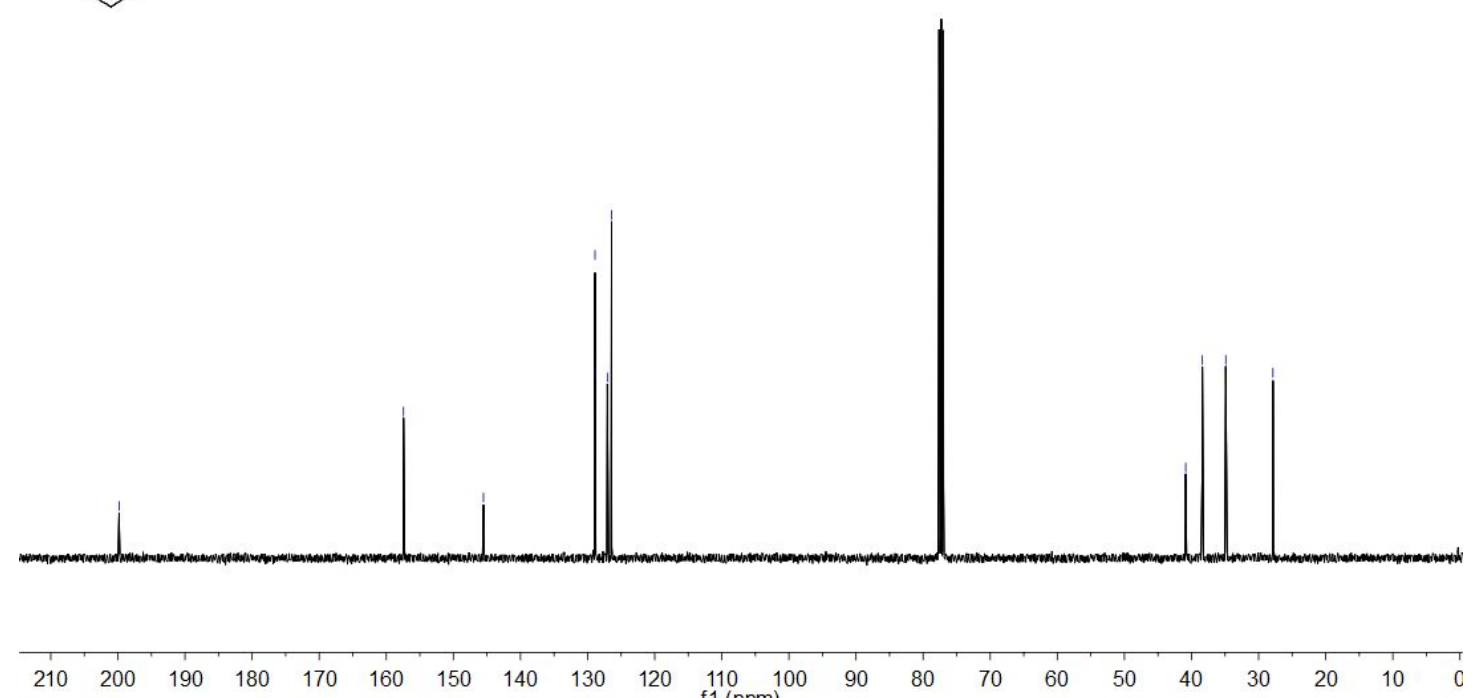
2b

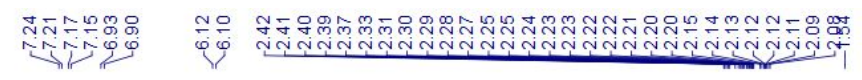<smiles>Cc1ccc([C@]2(C)C=CC(=O)CC2)cc1</smiles>

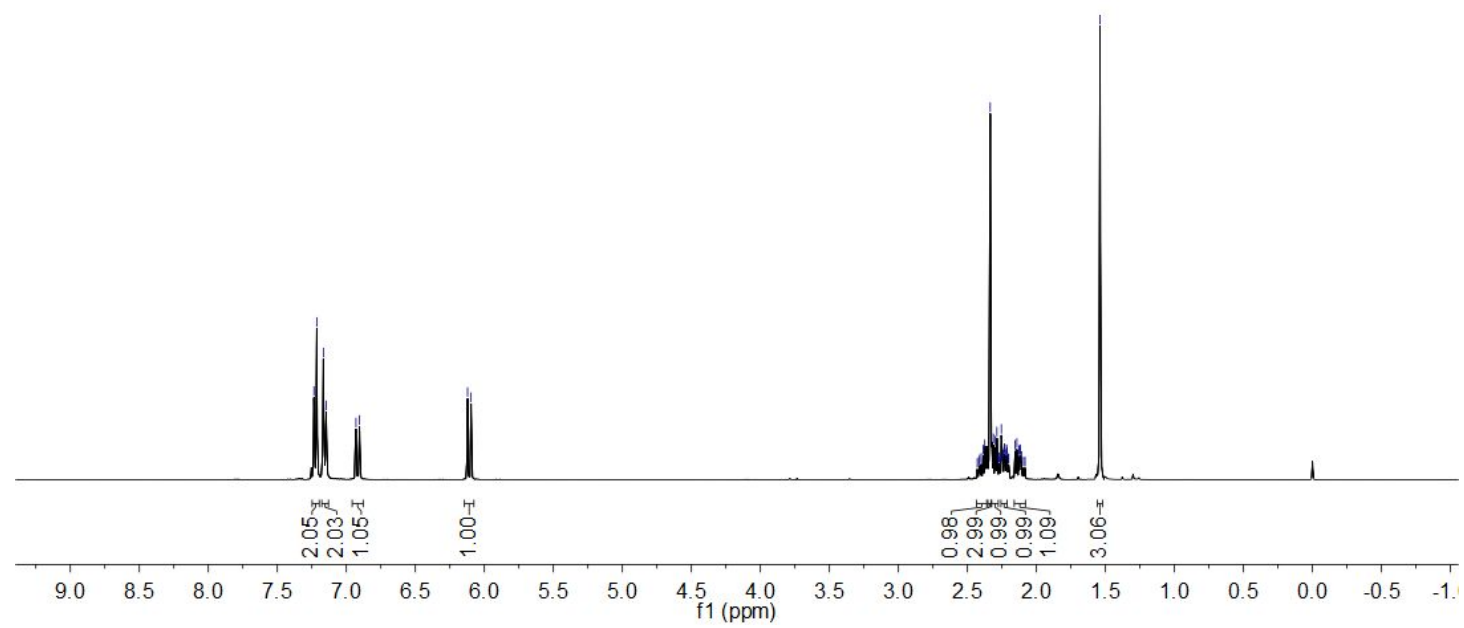

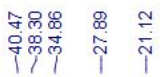<smiles>Cc1ccc([C@]2(C)C=CC(=O)CC2)cc1</smiles>

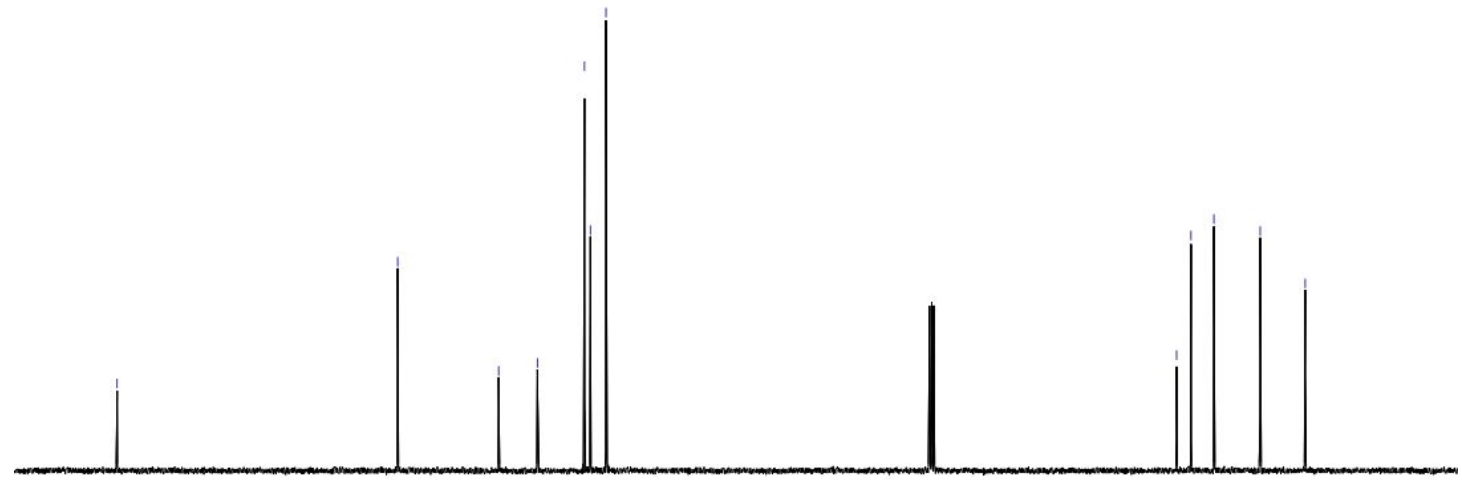

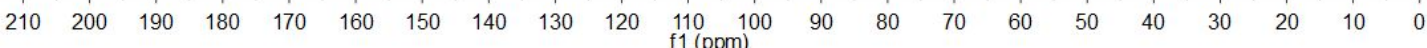




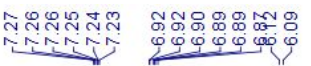

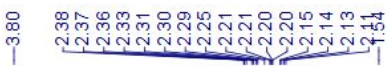<smiles>COc1ccc([C@]2(C)C=CC(=O)CC2)cc1</smiles>

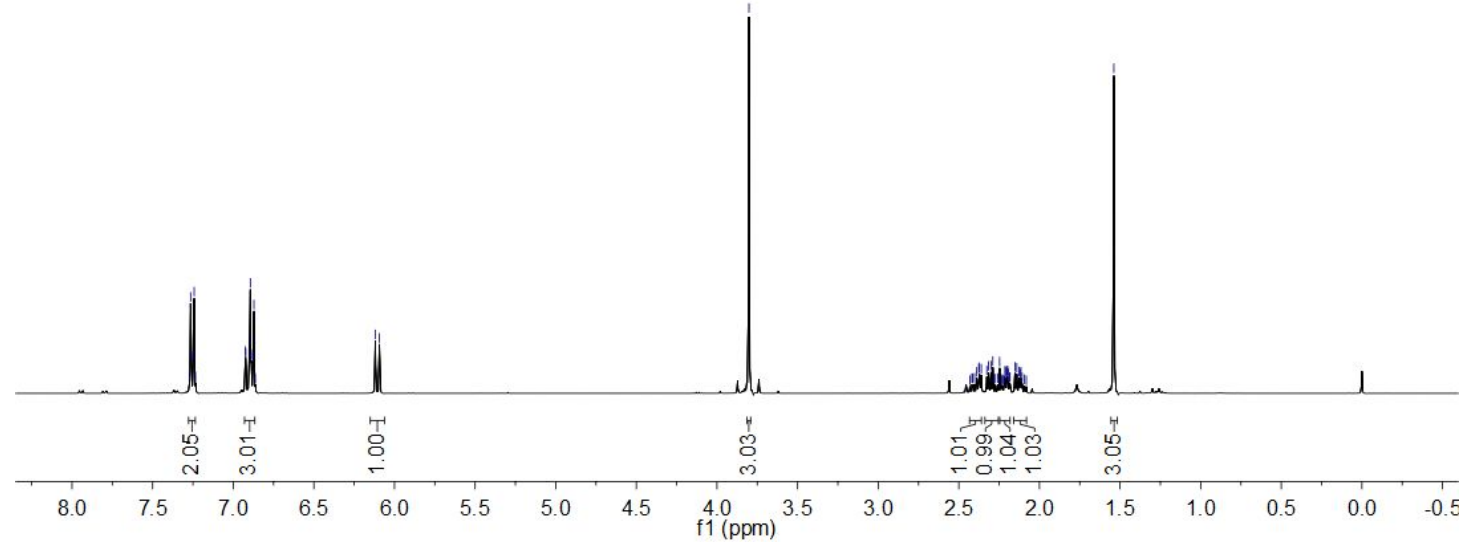

$\underset{\substack{\infty \\ \infty}}{\stackrel{\infty}{\infty}}$

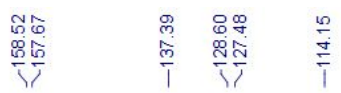

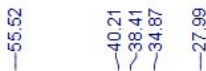<smiles>COc1ccc([C@]2(C)C=CC(=O)CC2)cc1</smiles>

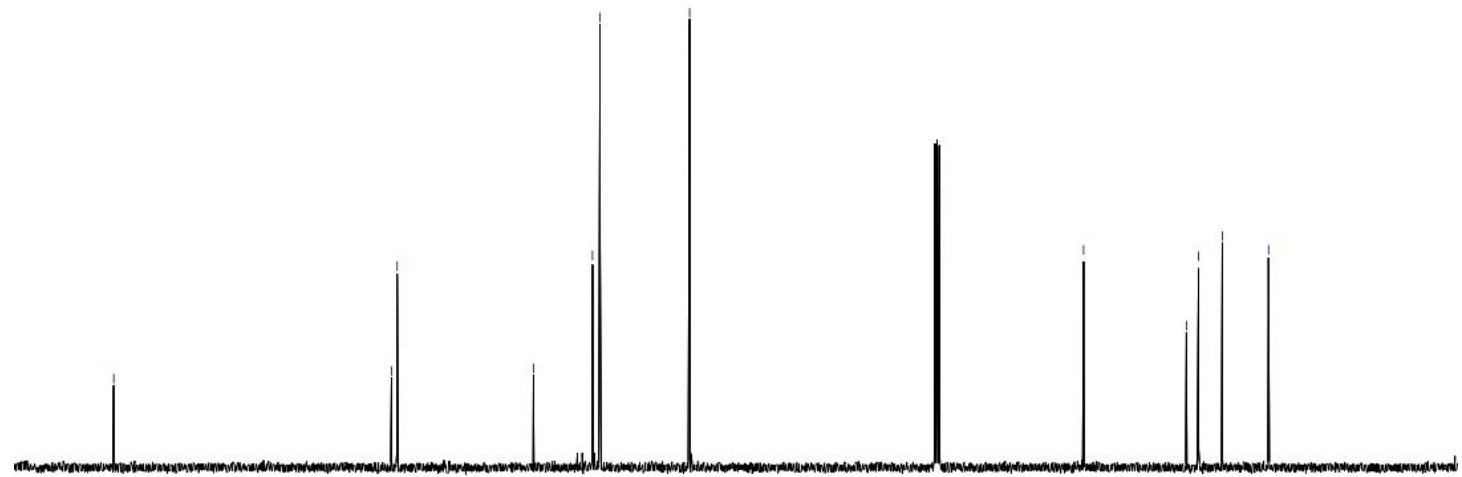

$\begin{array}{lllllllllllllllllllll}210 & 200 & 190 & 180 & 170 & 160 & 150 & 140 & 130 & 120 & 110 & 100 & 90 & 80 & 70 & 60 & 50 & 40 & 30 & 20 & 10\end{array}$ 


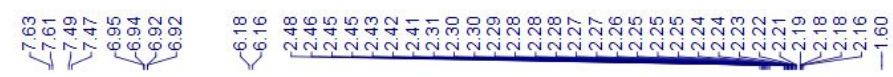<smiles>C[C@]1(c2ccc(C(F)(F)F)cc2)C=CC(=O)CC1</smiles>

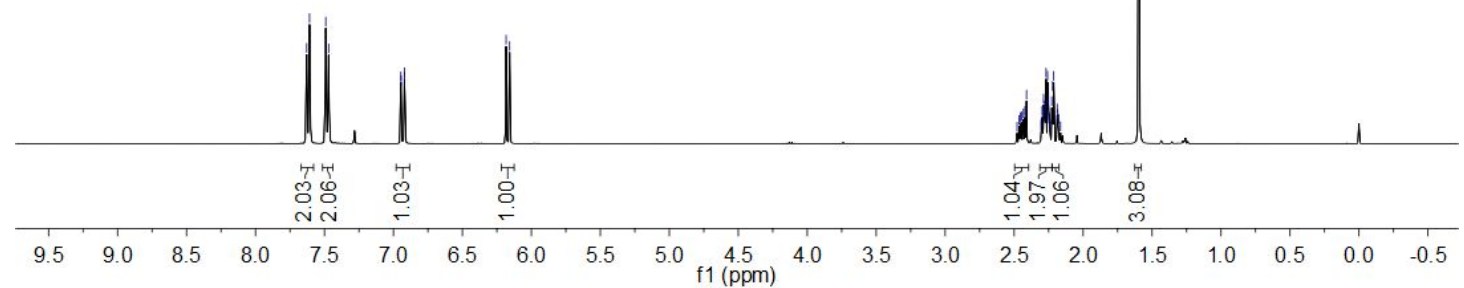

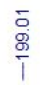

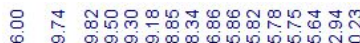

1

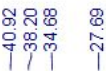<smiles>C[C@]1(c2ccc(C(F)(F)F)cc2)C=CC(=O)CC1</smiles>

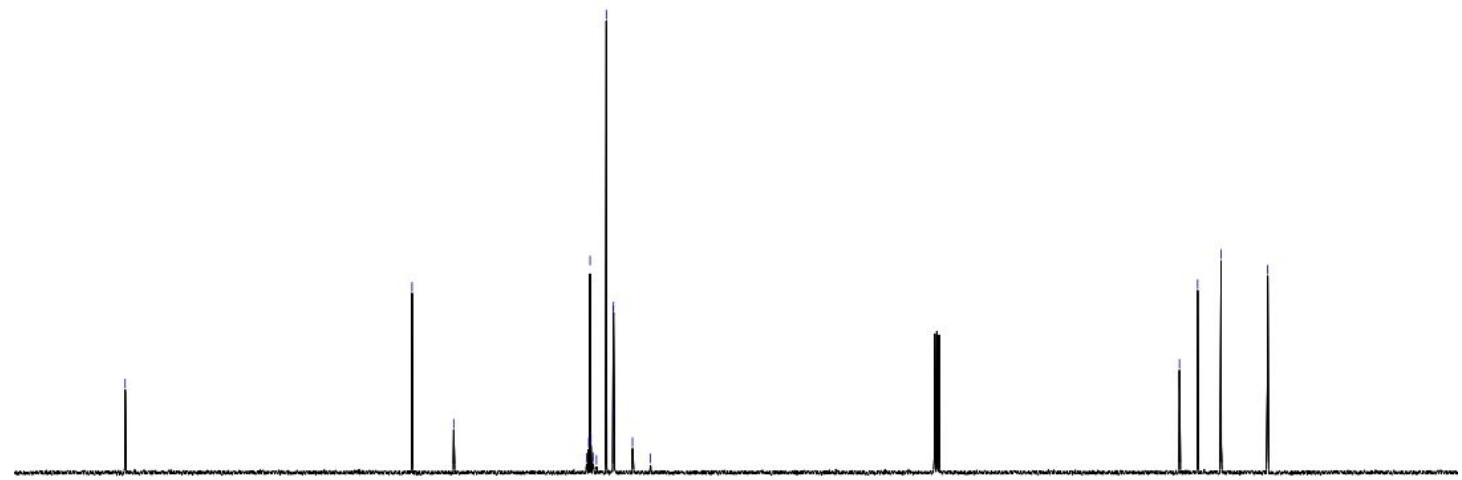

$\begin{array}{llllllllllllllllllllll}210 & 200 & 190 & 180 & 170 & 160 & 150 & 140 & 130 & 120 & \begin{array}{l}110 \\ \mathrm{f} 1(\mathrm{ppm})\end{array} & 100 & 90 & 80 & 70 & 60 & 50 & 40 & 30 & 20 & 10 & 0\end{array}$ 


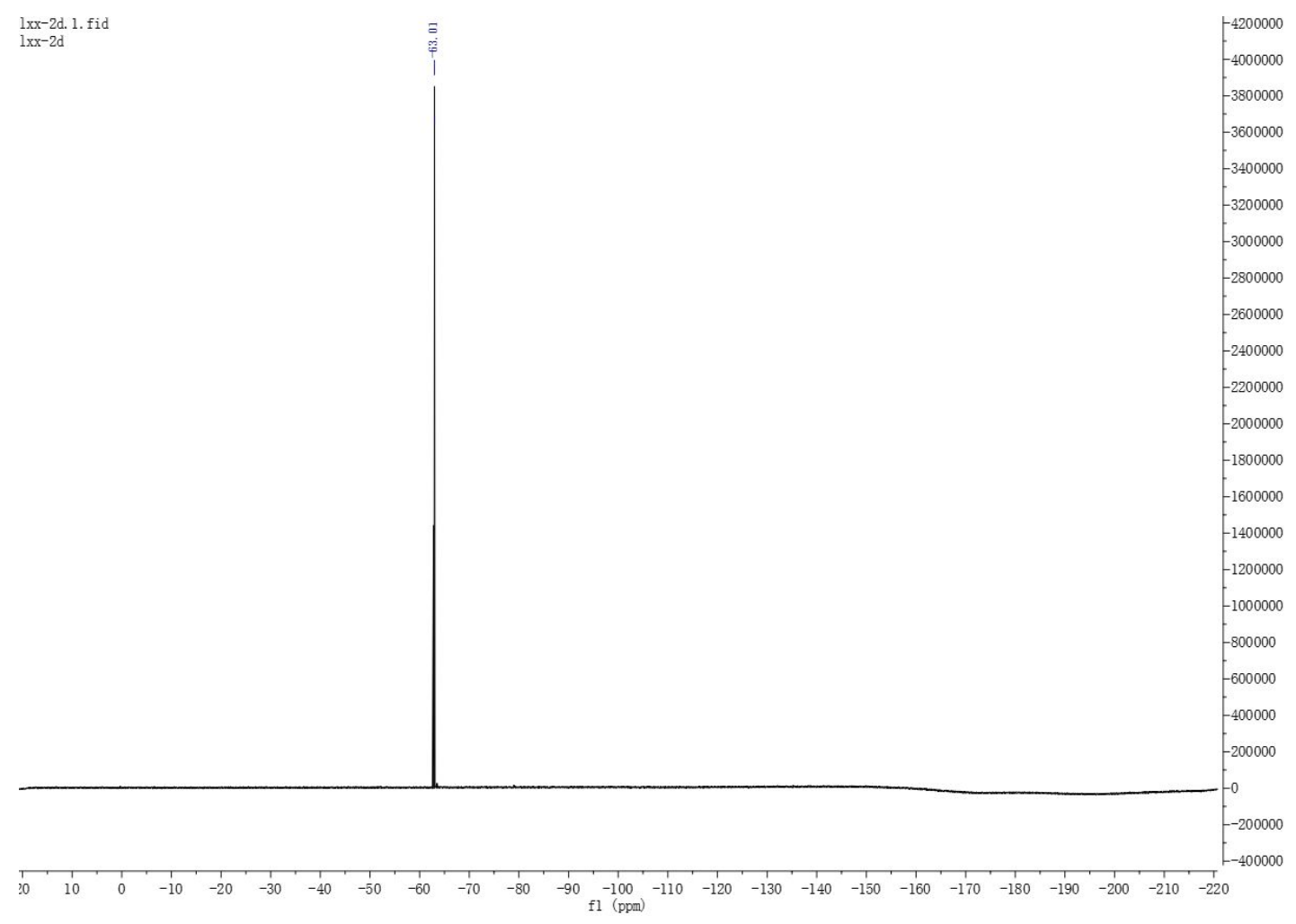




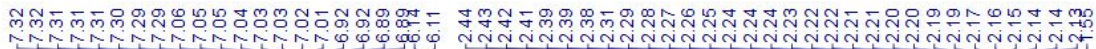

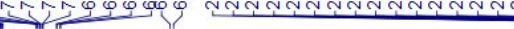<smiles>CC1(c2ccc(F)cc2)C=CC(=O)CC1</smiles>

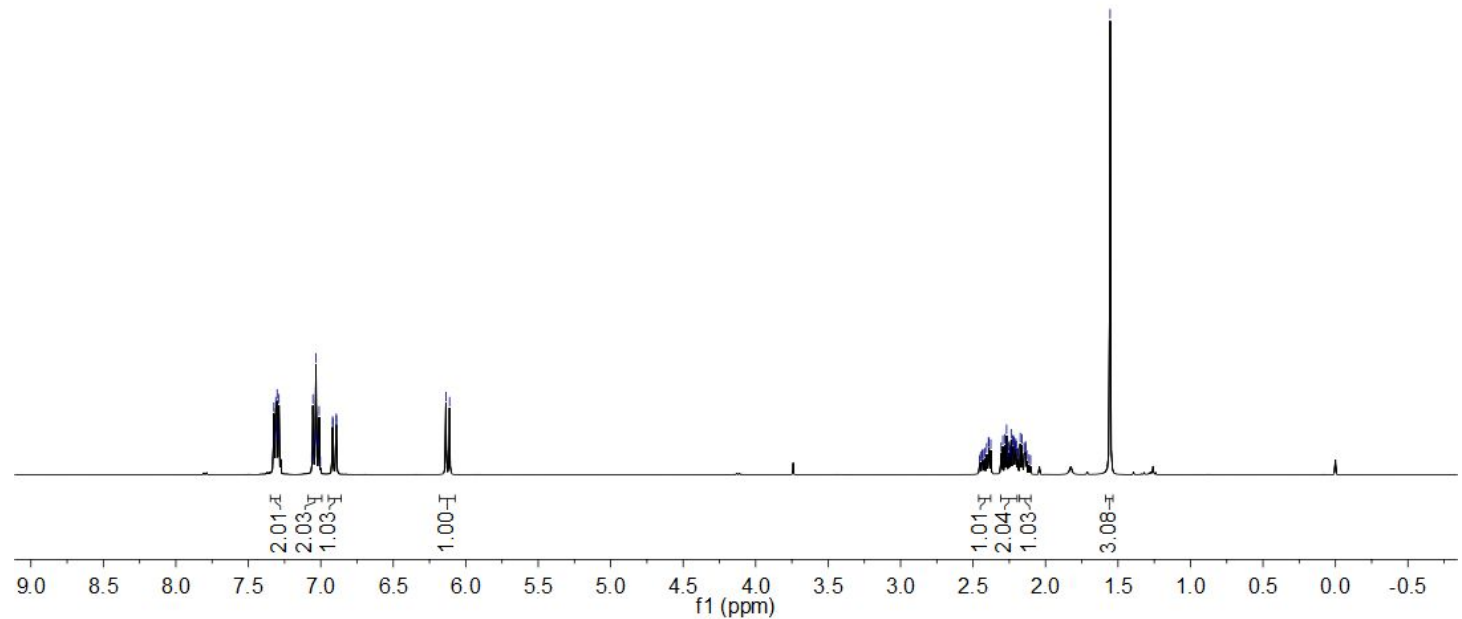

宁少<smiles>C[C@]1(c2ccc(F)cc2)C=CC(=O)CC1</smiles>

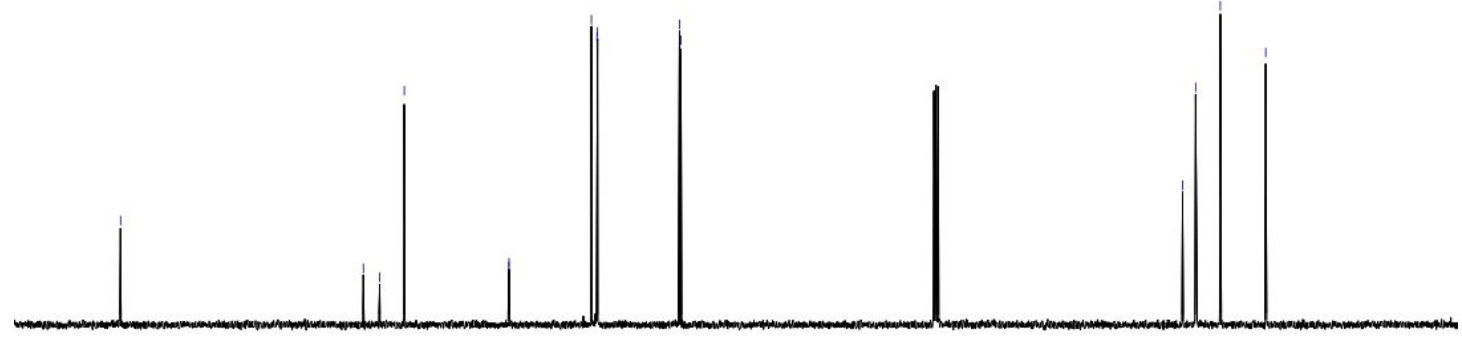

$\begin{array}{llllllllllllllllllllll}210 & 200 & 190 & 180 & 170 & 160 & 150 & 140 & 130 & 120 & \begin{array}{c}110 \\ \mathrm{f} 1(\mathrm{ppm})\end{array} & 100 & 90 & 80 & 70 & 60 & 50 & 40 & 30 & 20 & 10 & 0\end{array}$ 


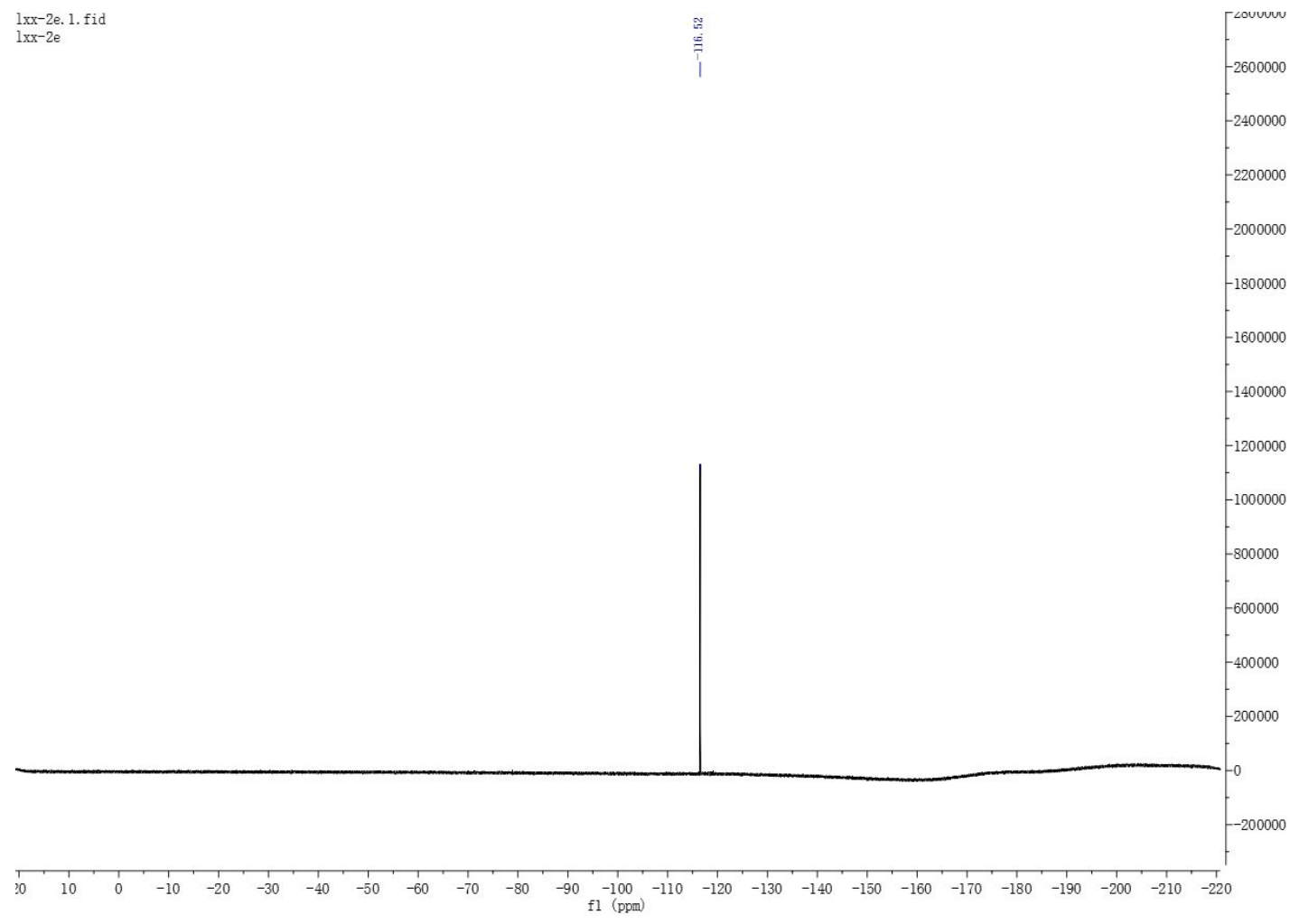




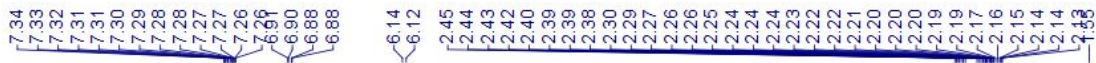<smiles>CC1(c2ccc(Cl)cc2)C=CC(=O)CC1</smiles>

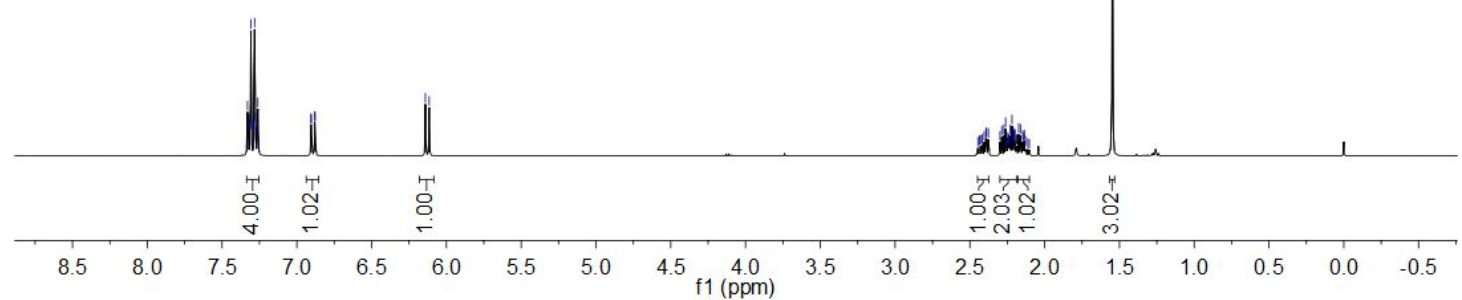

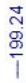<smiles>CC1(c2ccc(Cl)cc2)C=CC(=O)CC1</smiles>

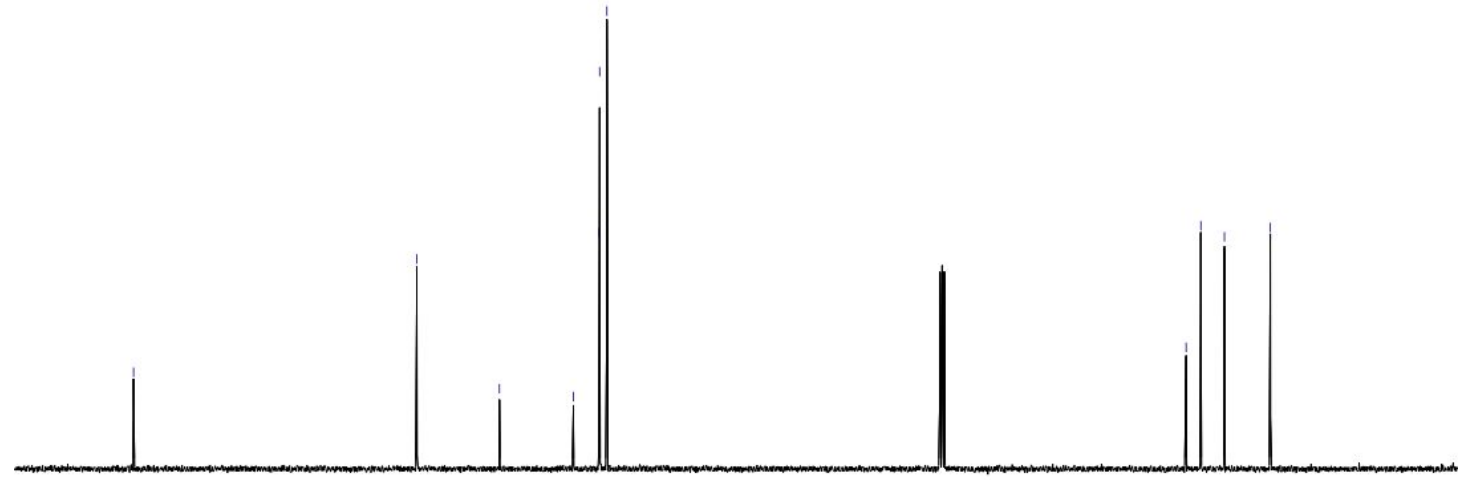

$\begin{array}{llllllllllllllllllllll}210 & 200 & 190 & 180 & 170 & 160 & 150 & 140 & 130 & 120 & 110 & 100 & 90 & 80 & 70 & 60 & 50 & 40 & 30 & 20 & 10 & 0\end{array}$ 
$2 g$

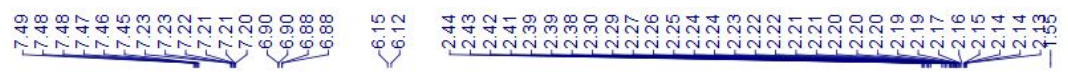<smiles>CC1(c2ccc(Br)cc2)C=CC(=O)CC1</smiles>

$\|$

$\mathrm{H}_{2} \mathrm{O}$

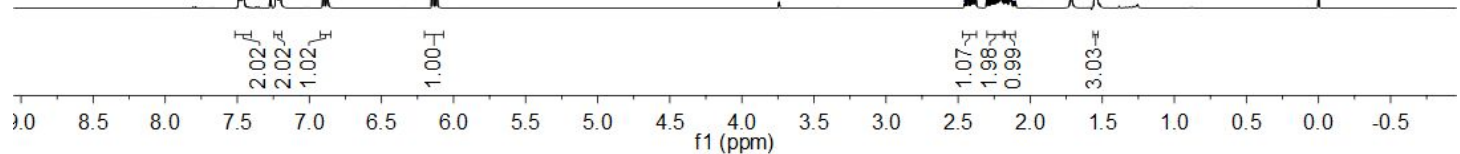

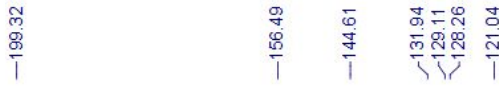

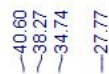<smiles>CC1(c2ccc(Br)cc2)C=CC(=O)CC1</smiles>

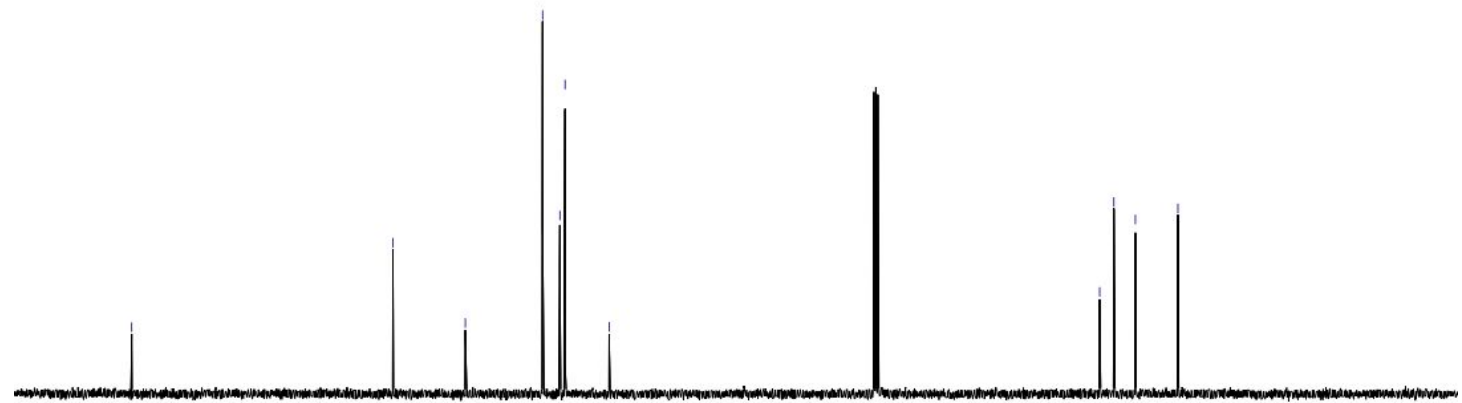

$\begin{array}{lllllllllllllllllllllll}210 & 200 & 190 & 180 & 170 & 160 & 150 & 140 & 130 & 120 & 110 & 100 & 90 & 80 & 70 & 60 & 50 & 40 & 30 & 20 & 10 & 0 & -10\end{array}$ 


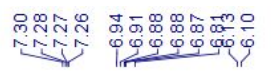

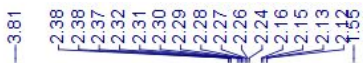<smiles>COc1cccc([C@]2(C)C=CC(=O)CC2)c1</smiles>

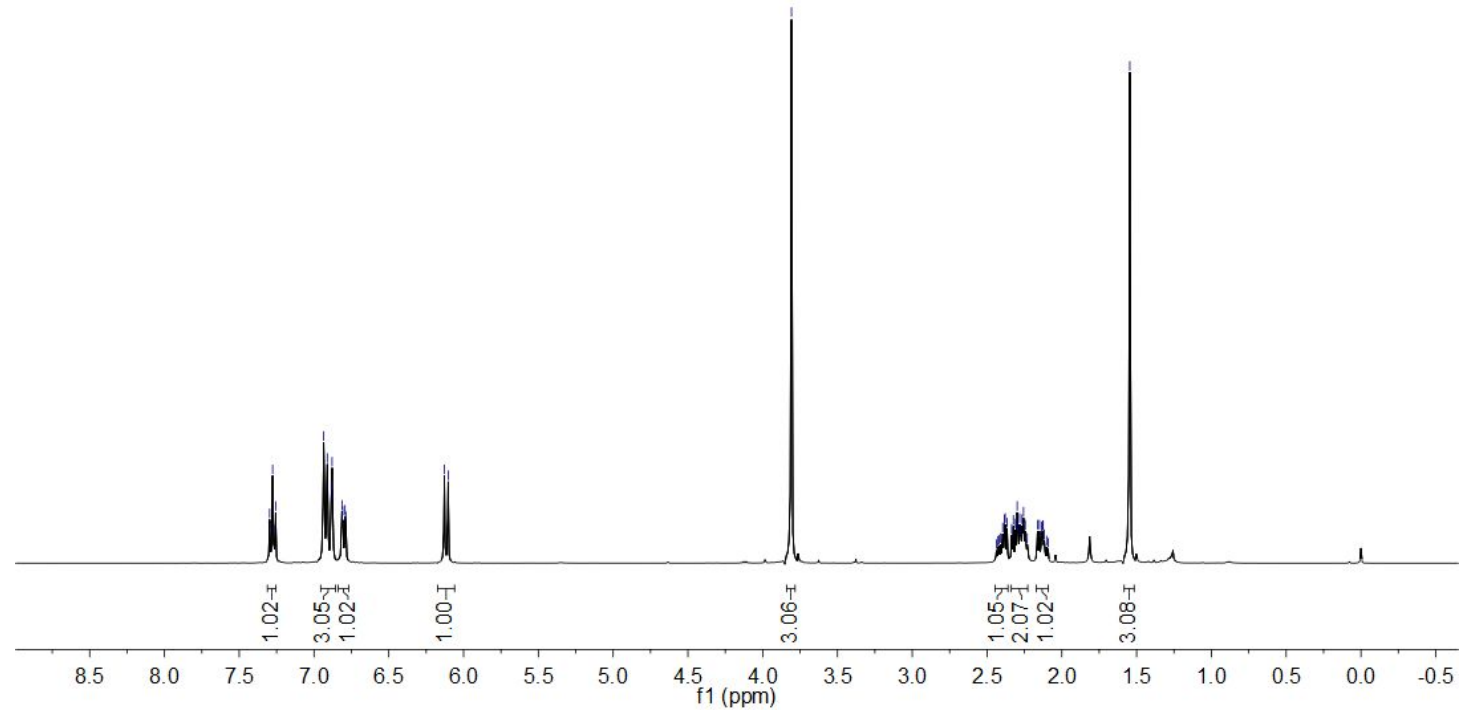<smiles>COc1cccc([C@]2(C)C=CC(=O)CC2)c1</smiles>

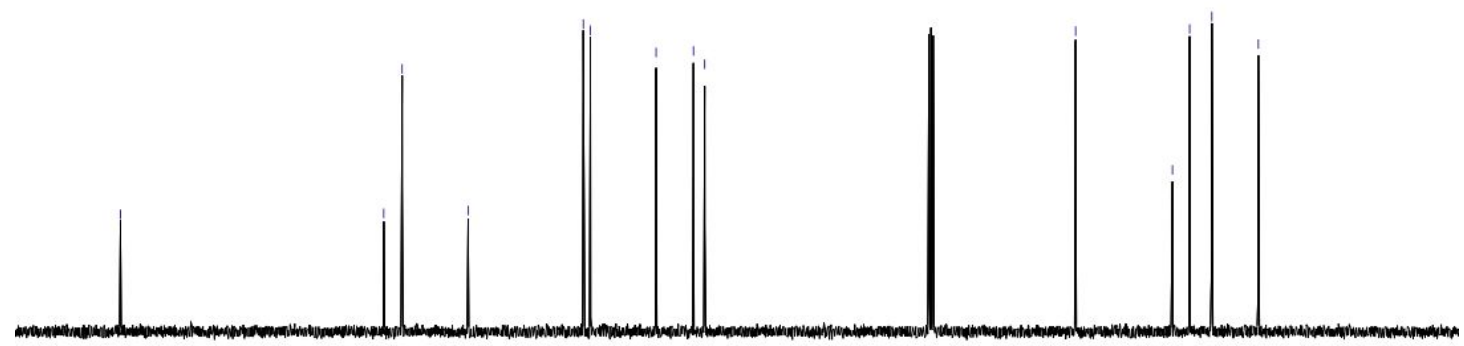

$\begin{array}{llllllllllllllllllllll}210 & 200 & 190 & 180 & 170 & 160 & 150 & 140 & 130 & 120 & \begin{array}{l}110 \\ \mathrm{f} 1(\mathrm{ppm})\end{array} & 90 & 80 & 70 & 60 & 50 & 40 & 30 & 20 & 10 & 0\end{array}$ 
<smiles>COc1ccccc1[C@]1(C)C=CC(=O)CC1</smiles>
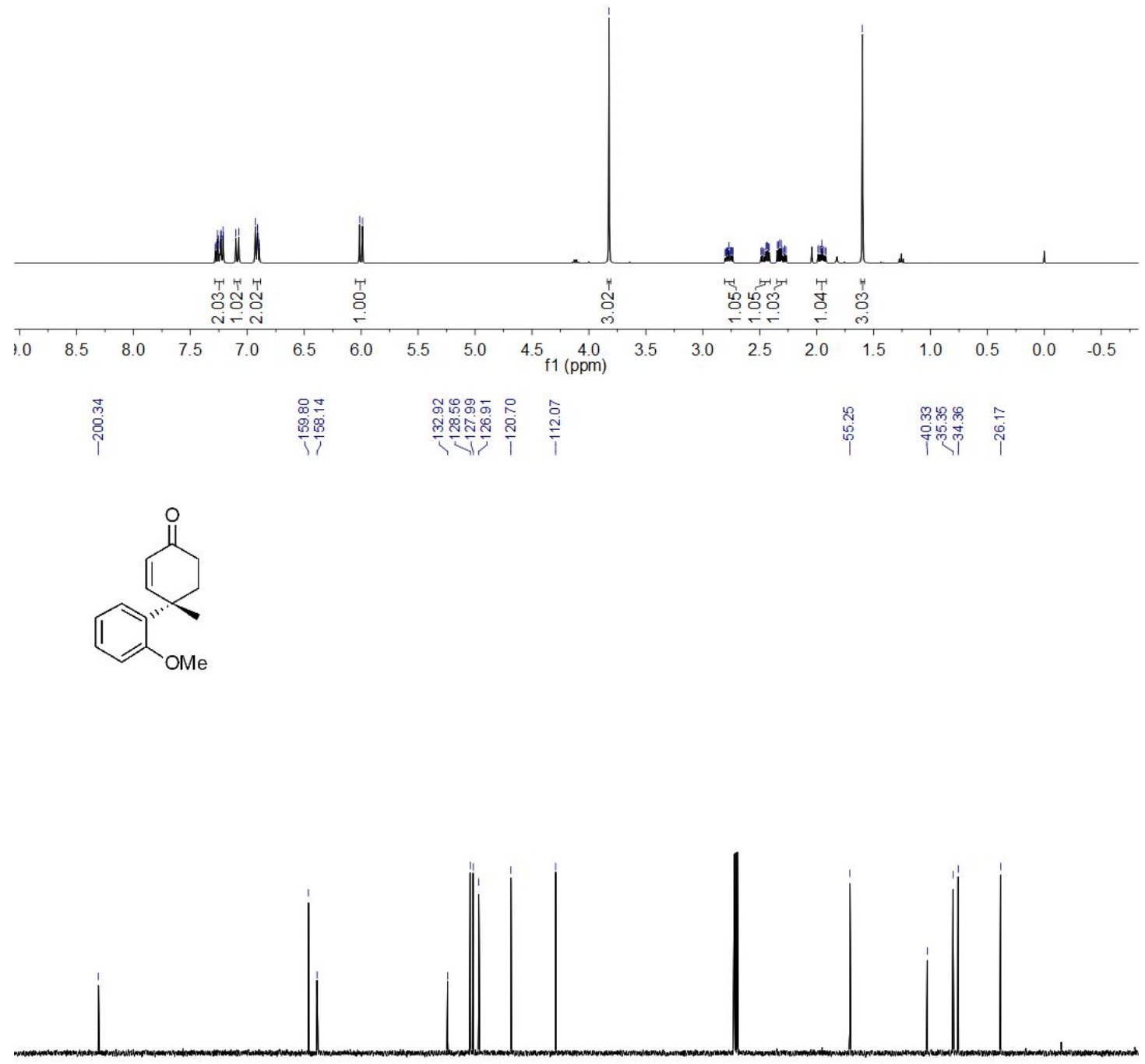

$\begin{array}{llllllllllllllllllllll}210 & 200 & 190 & 180 & 170 & 160 & 150 & 140 & 130 & 120 & \begin{array}{l}110 \\ \mathrm{f} 1(\mathrm{ppm})\end{array} & 100 & 90 & 80 & 70 & 60 & 50 & 40 & 30 & 20 & 10 & 0\end{array}$ 
$2 \mathrm{j}$

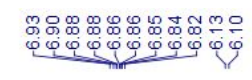

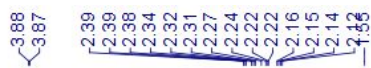

(II)

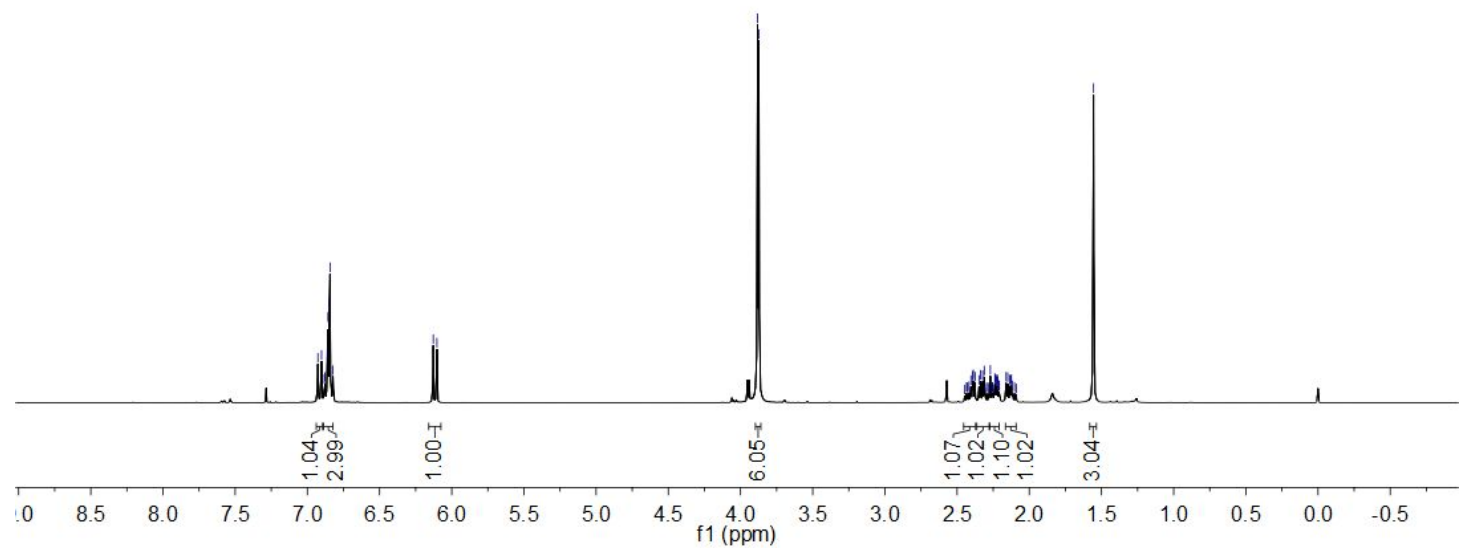

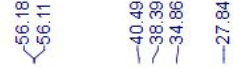<smiles>COc1ccc(C2(C)C=CC(=O)CC2)cc1OC</smiles>

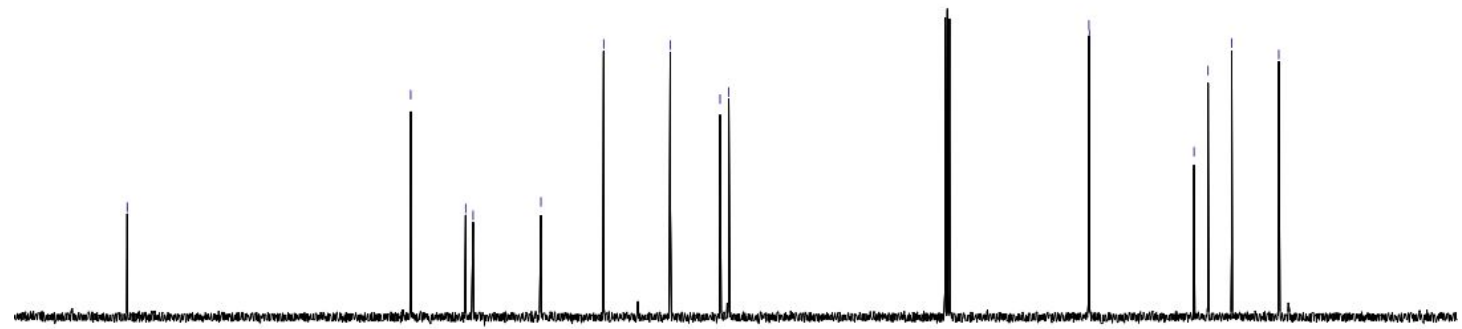

$\begin{array}{lllllllllllllllllllll}210 & 200 & 190 & 180 & 170 & 160 & 150 & 140 & 130 & 120 & 110 & 100 & 90 & 80 & 70 & 60 & 50 & 40 & 30 & 20 & 10\end{array}$ 
$2 \mathbf{k}$

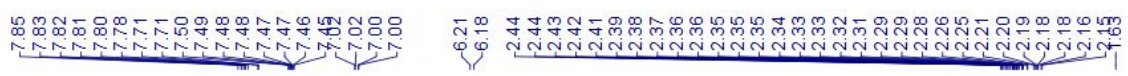<smiles>C[C@]1(c2ccc3ccccc3c2)C=CC(=O)CC1</smiles>

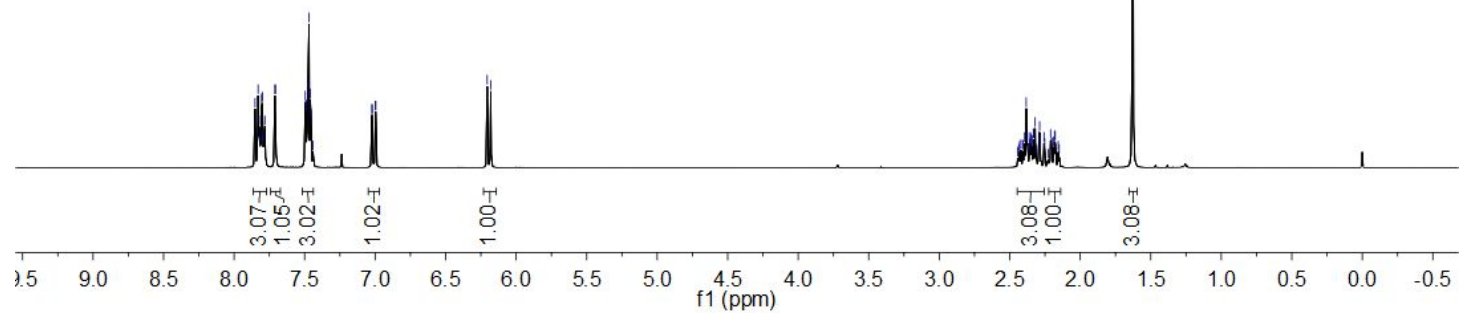

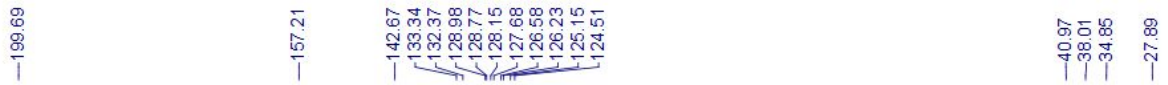<smiles>CC1(C2C=Cc3ccccc3C=C2)C=CC(=O)CC1</smiles>

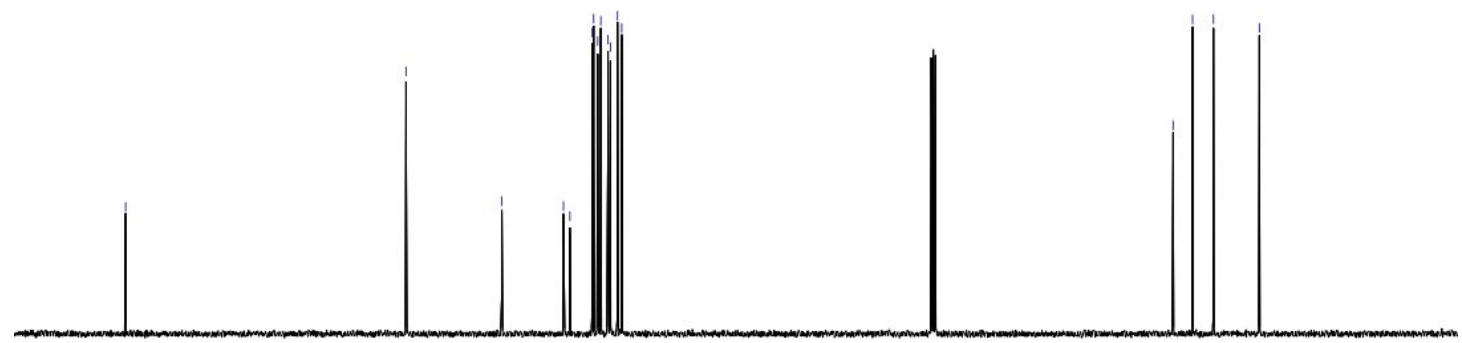

$\begin{array}{llllllllllllllllllllll}210 & 200 & 190 & 180 & 170 & 160 & 150 & 140 & 130 & 120 & \begin{array}{c}110 \\ \mathrm{f} 1(\mathrm{ppm})\end{array} & 100 & 90 & 80 & 70 & 60 & 50 & 40 & 30 & 20 & 10 & 0\end{array}$ 


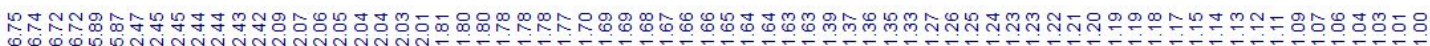<smiles>CC1(C2CCCCC2)C=CC(=O)CC1</smiles>

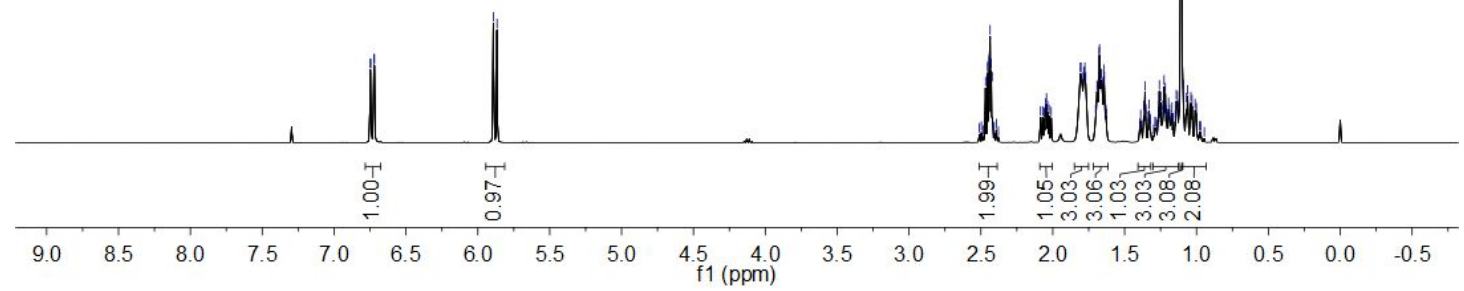

융

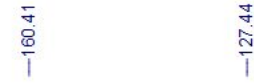

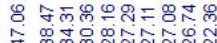<smiles>C[C@]1(C2CCCCC2)C=CC(=O)CC1</smiles>

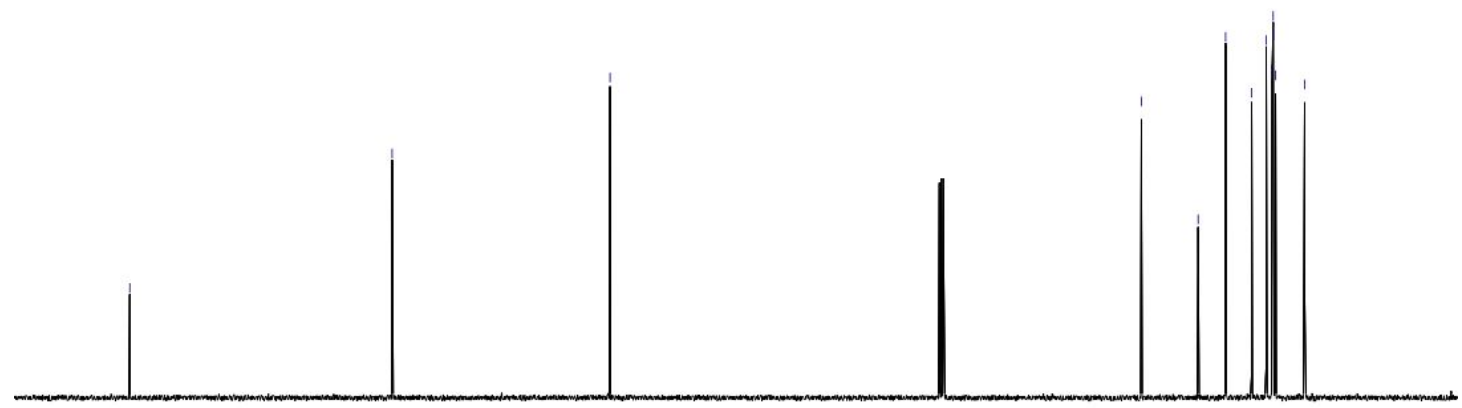

$\begin{array}{llllllllllllllllllllll}210 & 200 & 190 & 180 & 170 & 160 & 150 & 140 & 130 & 120 & \begin{array}{c}110 \\ \mathrm{f} 1(\mathrm{ppm})\end{array} & 100 & 90 & 80 & 70 & 60 & 50 & 40 & 30 & 20 & 10 & 0\end{array}$ 


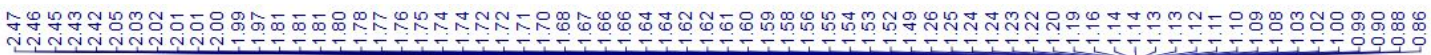<smiles>CC[C@]1(C2CCCCC2)C=CC(=O)CC1</smiles>

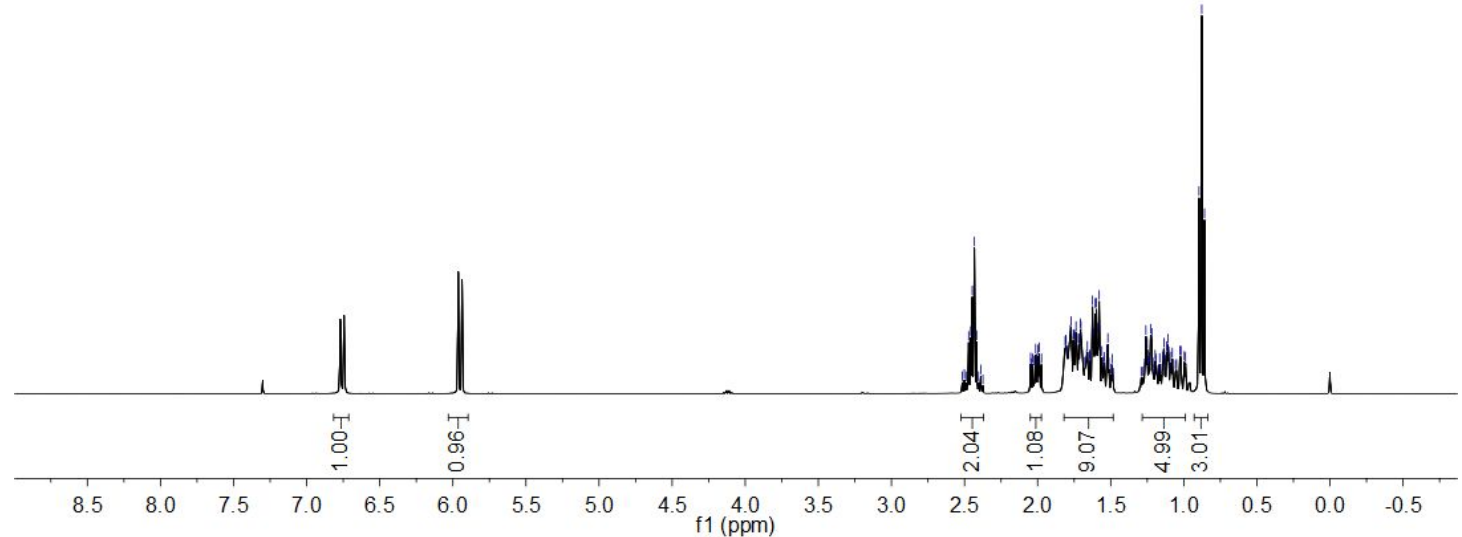

ঙำ

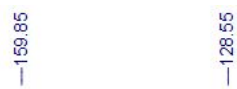

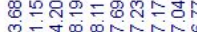

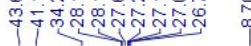<smiles>CCC1(C2CCCCC2)C=CC(=O)CC1</smiles>

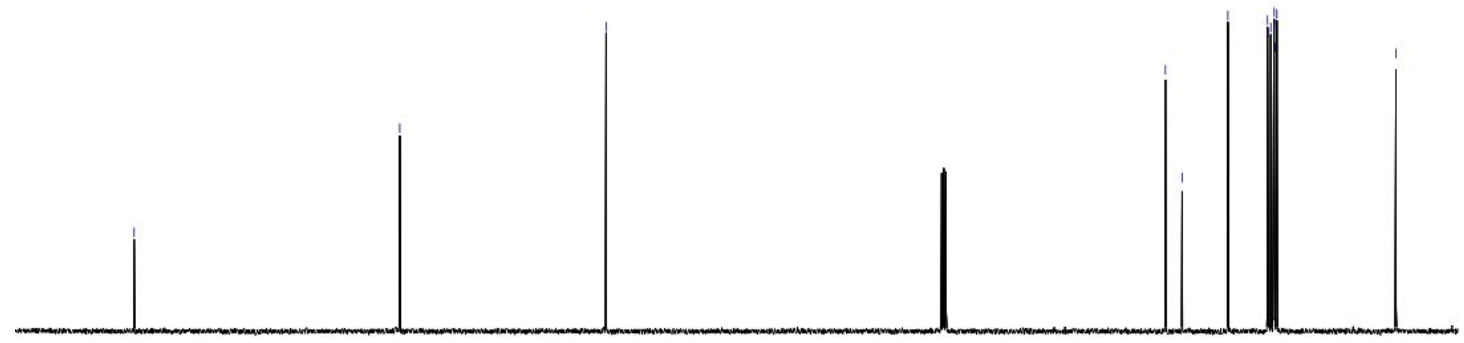

$\begin{array}{llllllllllllllllllllll}210 & 200 & 190 & 180 & 170 & 160 & 150 & 140 & 130 & 120 & \begin{array}{l}110 \\ 10\end{array} & 100 & 90 & 80 & 70 & 60 & 50 & 40 & 30 & 20 & 10 & 0\end{array}$ 
<smiles>O=C1C=C[C@]2(CC1)CCc1ccccc12</smiles>

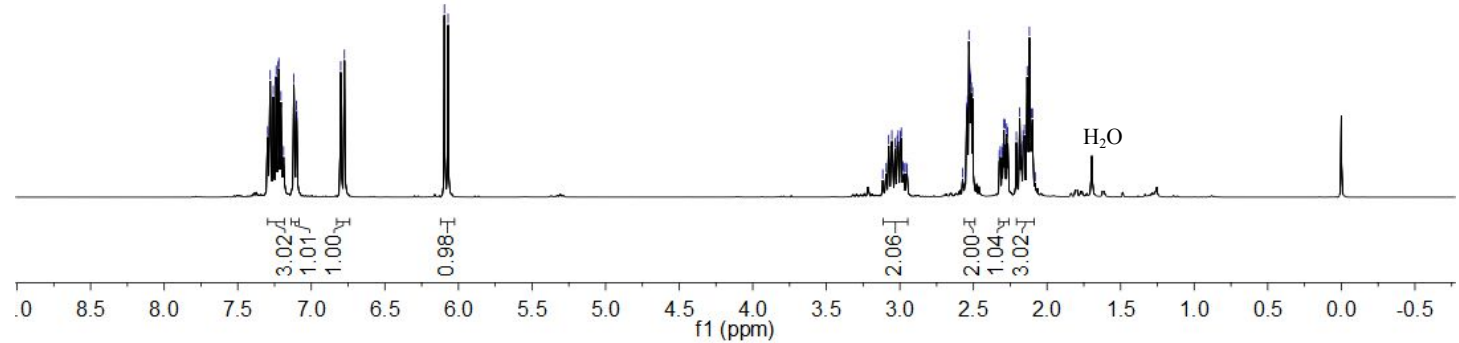<smiles>O=C1C=C[C@]2(CC1)CCc1ccccc12</smiles>

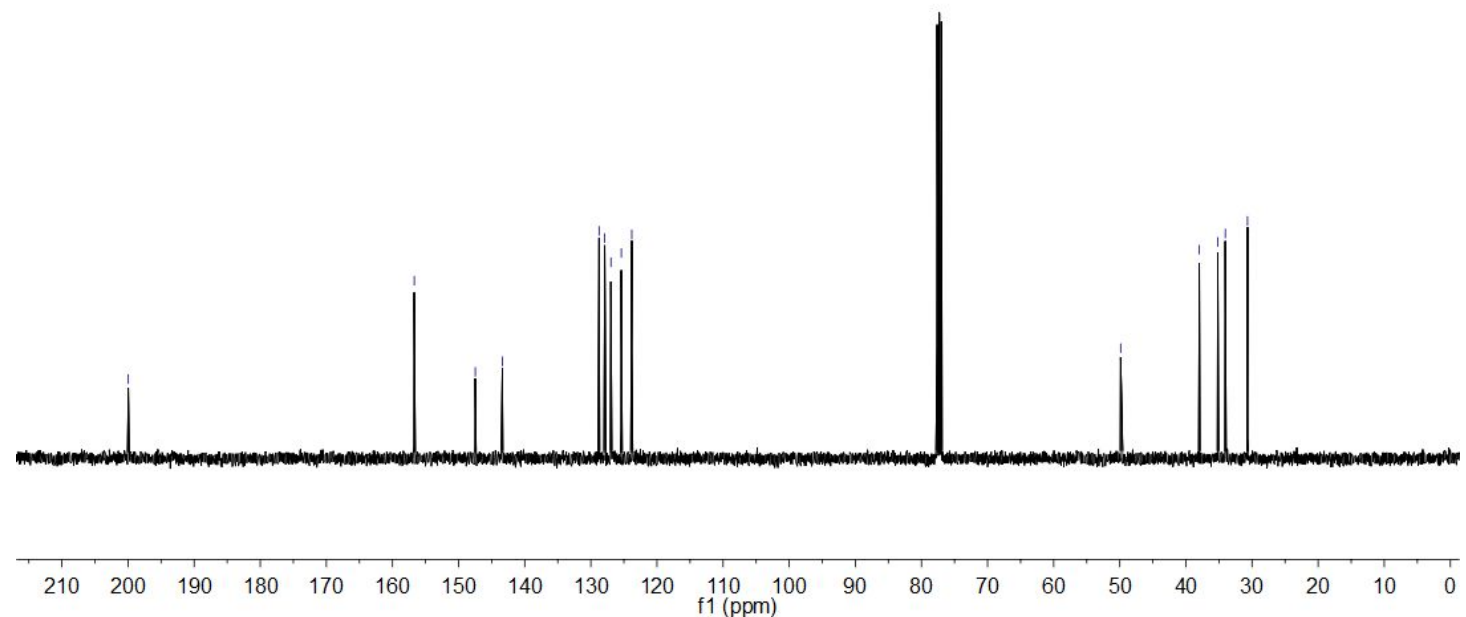


$4 b$

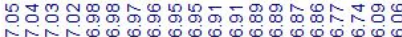

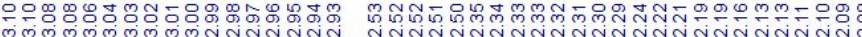<smiles>O=C1C=C[C@]2(CC1)CCc1cc(F)ccc12</smiles>

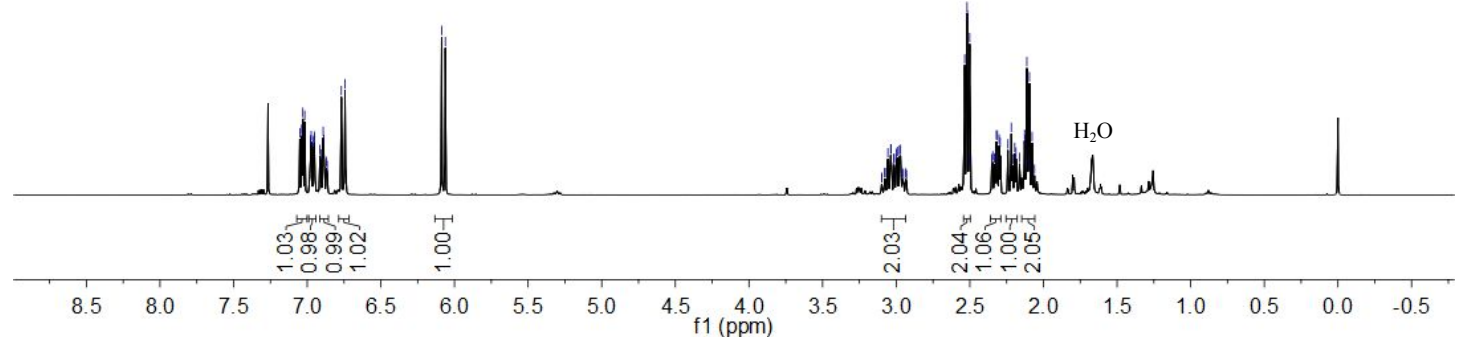<smiles>O=C1C=C[C@]2(CC1)CCc1cc(F)ccc12</smiles>

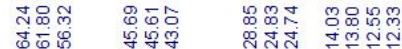

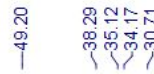

广

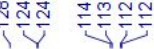

$\mathrm{F}$

$\begin{array}{lllllllllllllllllllll}210 & 200 & 190 & 180 & 170 & 160 & 150 & 140 & 130 & 120 & 110 & 100 & 90 & 80 & 70 & 60 & 50 & 40 & 30 & 20 & 10\end{array}$ 
lxx-4b. 1. fid

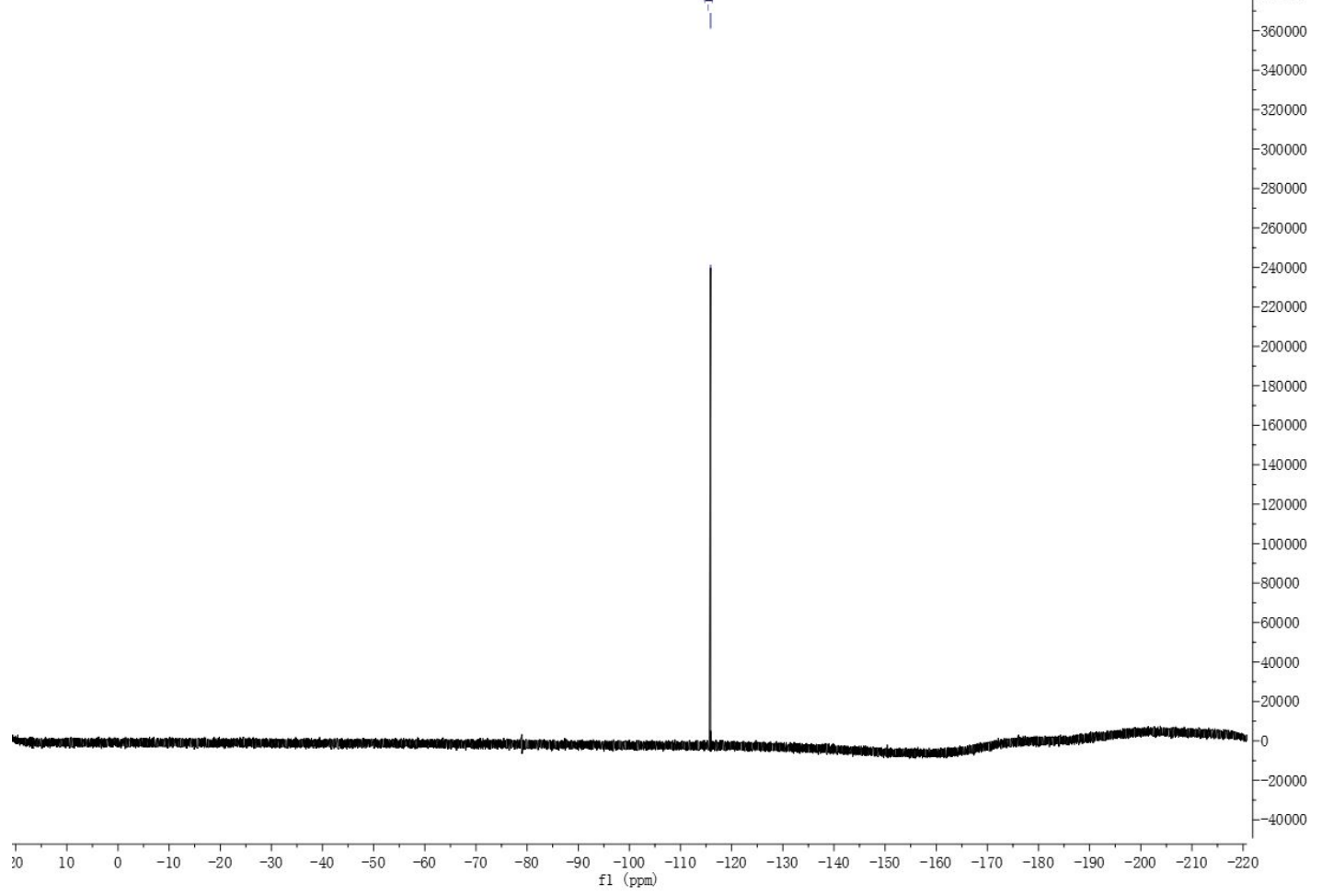


4c

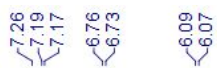

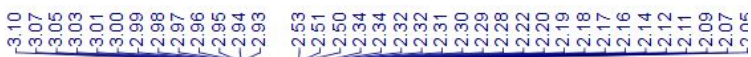<smiles>Oc1ccc(Cl)cc1CC1CC2CCC(C2)C1</smiles>

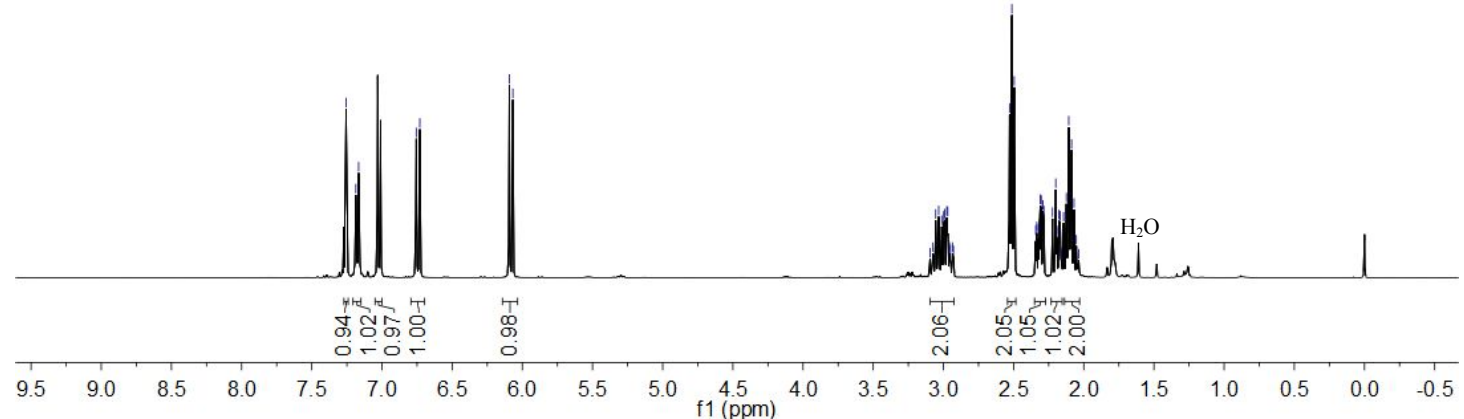<smiles>O=C1CCC2(C=CC(Cl)CC2)CC1</smiles>

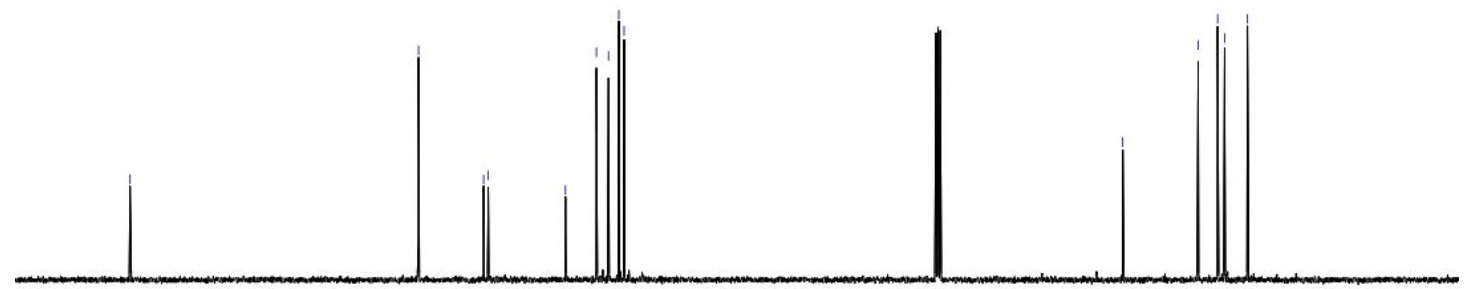

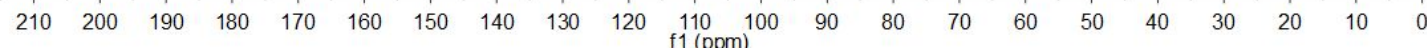




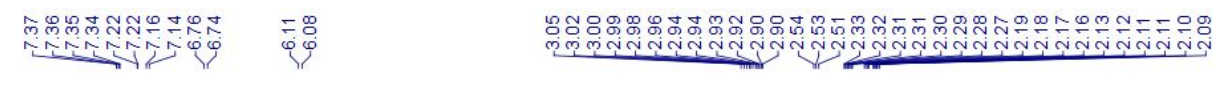<smiles>O=C1C=C[C@]2(CC1)CCc1ccc(Br)cc12</smiles>

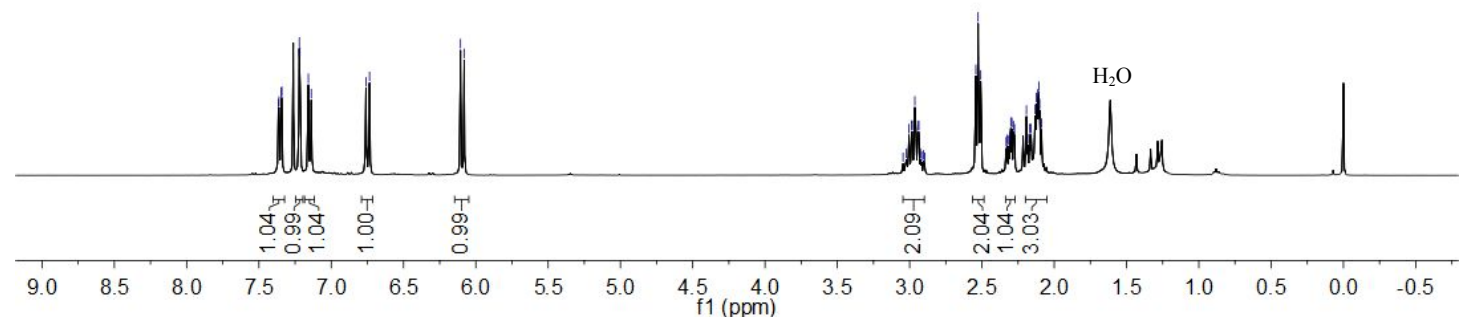

$\underset{\substack{\sigma \\ \stackrel{\sigma}{i}}}{\bar{\sigma}}$

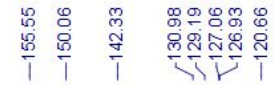

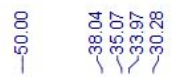<smiles>O=C1C=C[C@]2(CC1)CCc1ccc(Br)cc12</smiles>

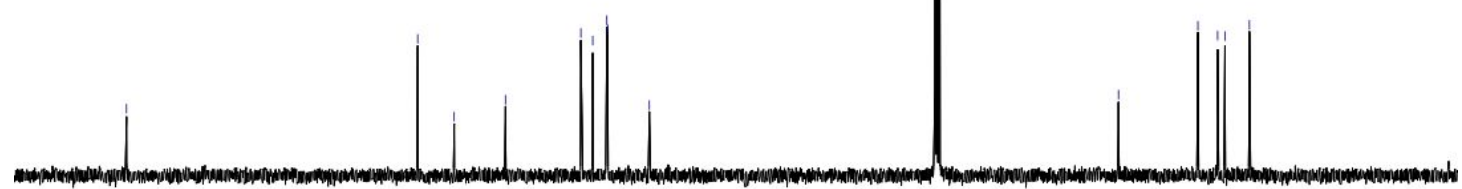

$\begin{array}{llllllllllllllllllllll}210 & 200 & 190 & 180 & 170 & 160 & 150 & 140 & 130 & 120 & \begin{array}{l}110 \\ \mathrm{f} 1(\mathrm{ppm})\end{array} & 100 & 90 & 80 & 70 & 60 & 50 & 40 & 30 & 20 & 10 & 0\end{array}$ 
$4 \mathbf{e}$
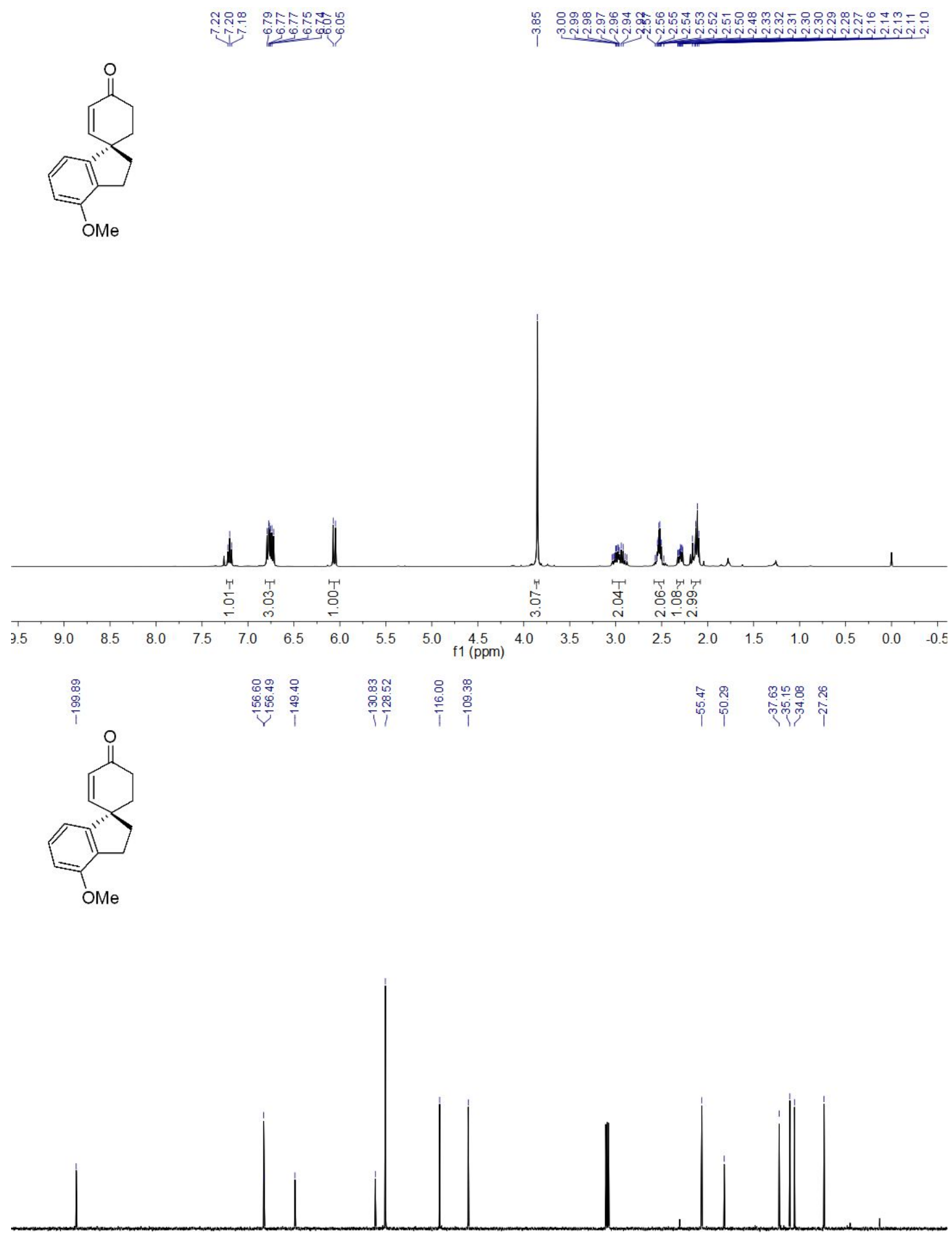

$\begin{array}{lllllllllllllllllllllllllll}210 & 200 & 190 & 180 & 170 & 160 & 150 & 140 & 130 & 120 & 110 & 100 & 90 & 80 & 70 & 60 & 50 & 40 & 30 & 20 & 10 & 0\end{array}$ 


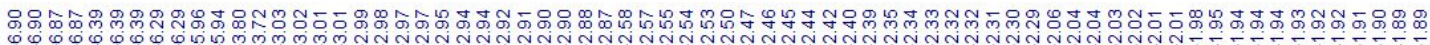<smiles>COc1cc2c(c(OC)c1)[C@]1(C=CC(=O)CC1)CC2</smiles>

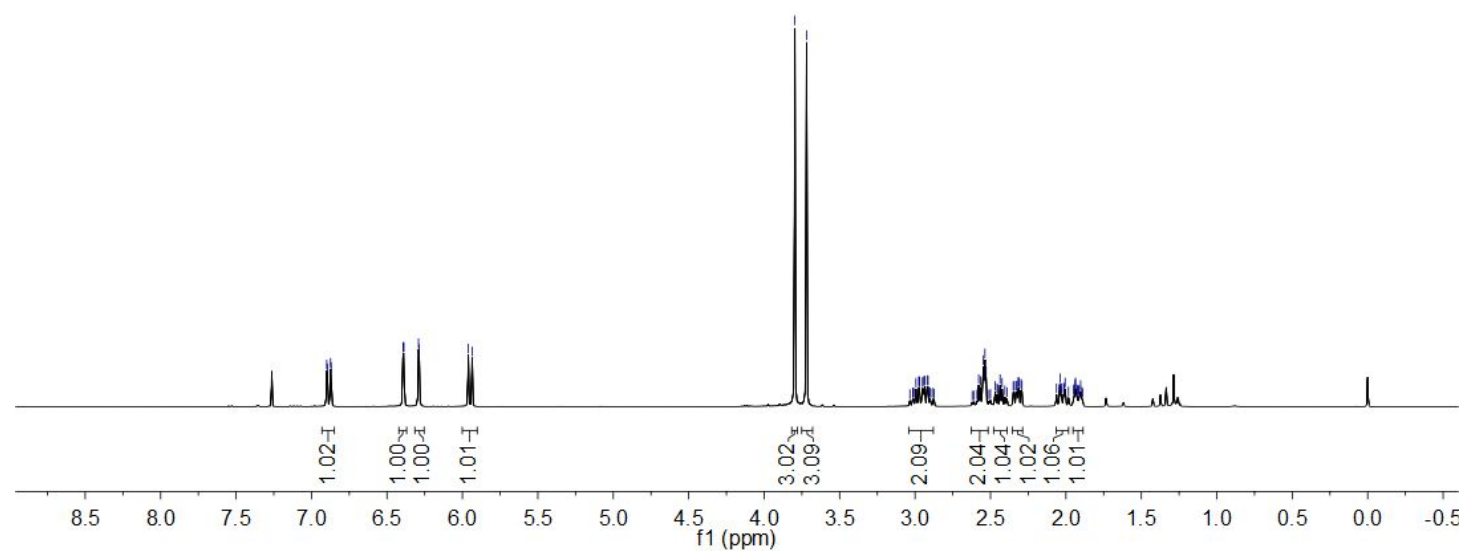<smiles>COc1cc2c(c(OC)c1)[C@]1(C=CC(=O)CC1)CC2</smiles>

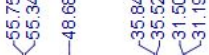

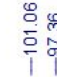

रुण

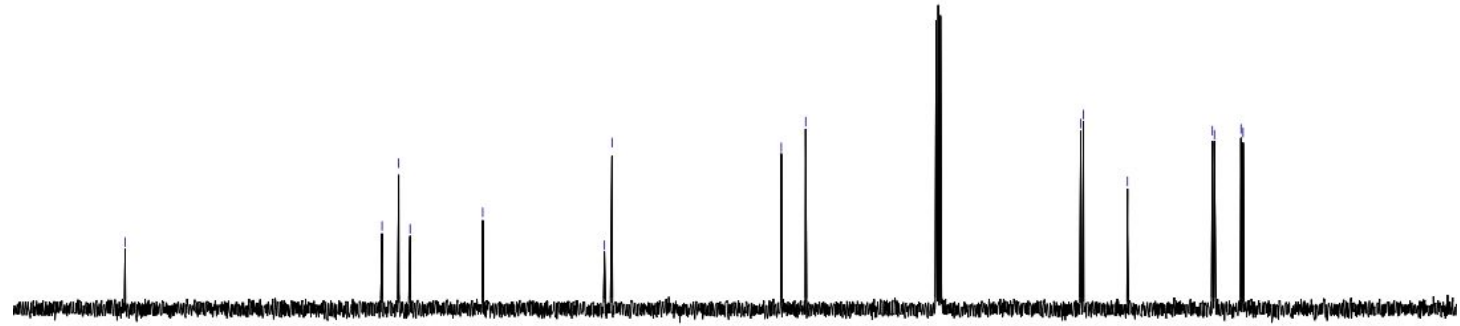

$\begin{array}{llllllllllllllllllllll}210 & 200 & 190 & 180 & 170 & 160 & 150 & 140 & 130 & 120 & \begin{array}{c}110 \\ 100\end{array} & 90 & 80 & 70 & 60 & 50 & 40 & 30 & 20 & 10 & 0\end{array}$ 


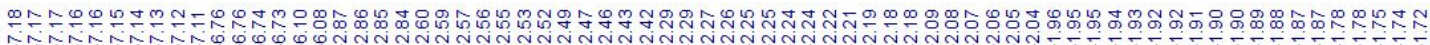<smiles>O=C1C=CC2(CCCc3ccccc32)CC1</smiles>

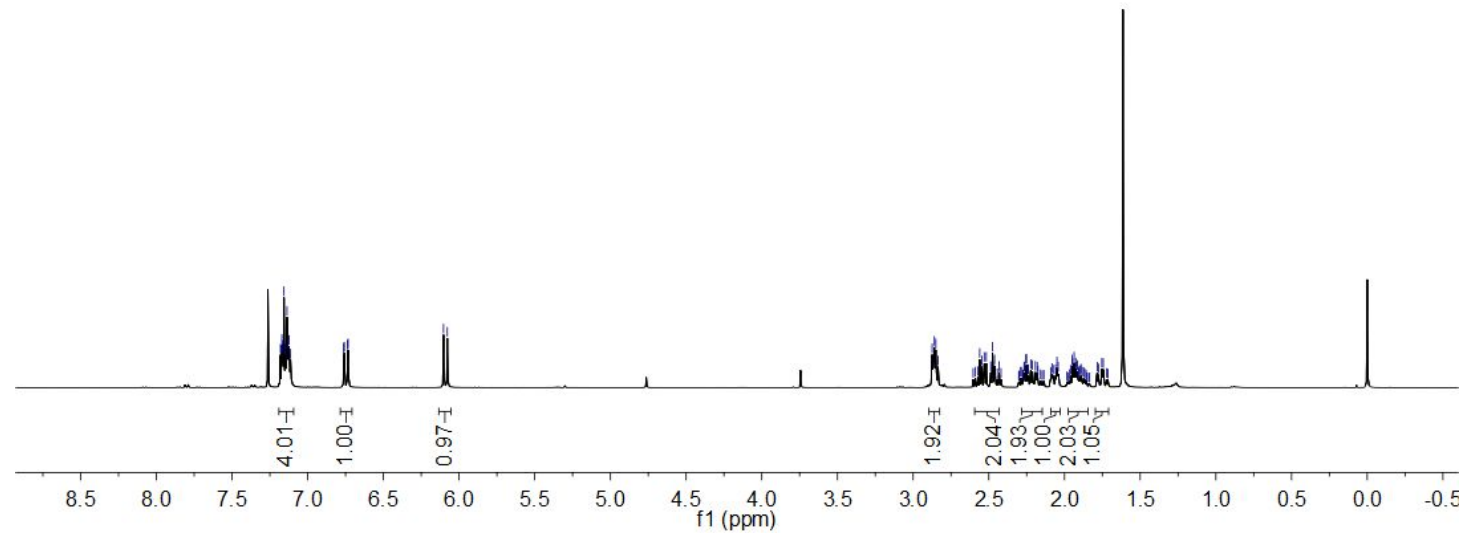<smiles>O=C1C=CC2(CCCc3ccccc32)c2ccccc21</smiles>

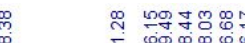

产

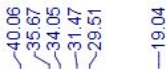

in

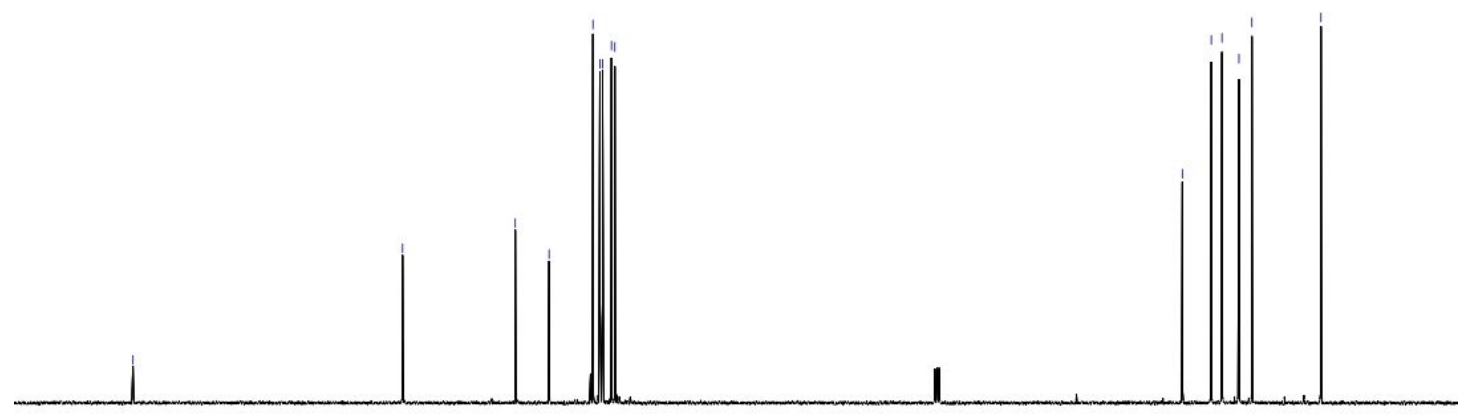

$\begin{array}{llllllllllllllllllllll}210 & 200 & 190 & 180 & 170 & 160 & 150 & 140 & 130 & 120 & \begin{array}{c}110 \\ \mathrm{f} 1(\mathrm{ppm})\end{array} & 100 & 90 & 80 & 70 & 60 & 50 & 40 & 30 & 20 & 10 & 0\end{array}$ 


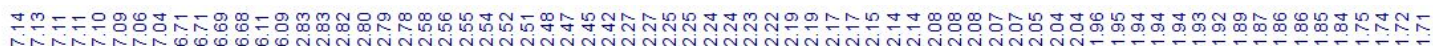<smiles>O=C1C=C[C@]2(CCCc3ccc(Cl)cc32)CC1</smiles>

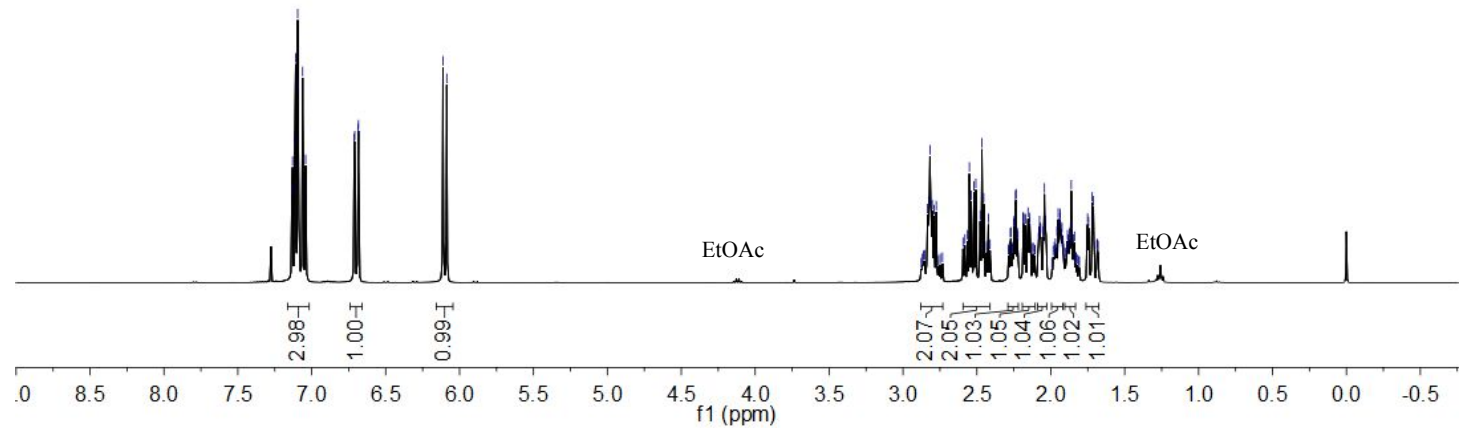<smiles>O=C1C=C[C@]2(CCCc3ccc(Cl)cc32)CC1</smiles>

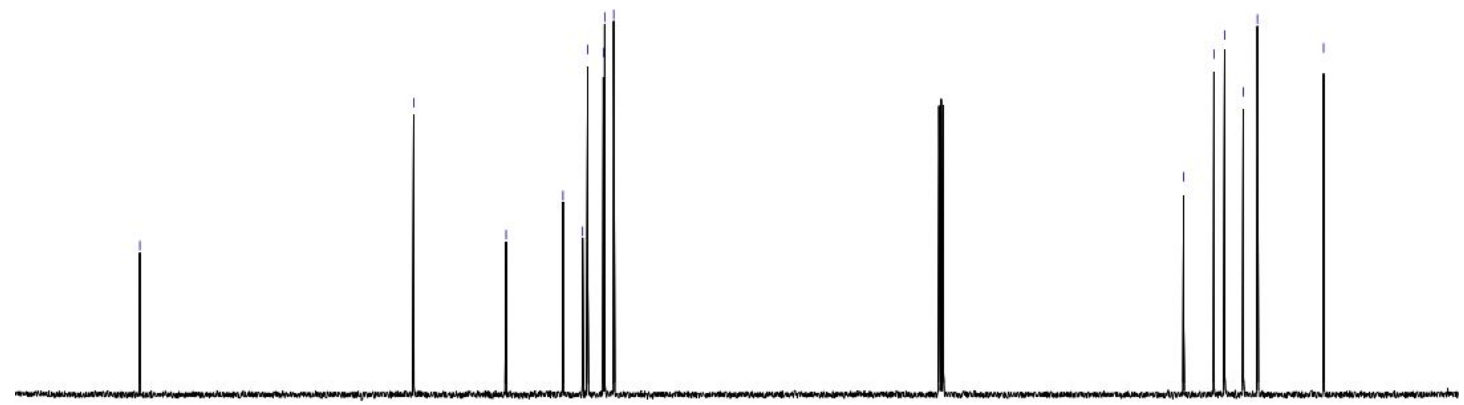

$\begin{array}{llllllllllllllllllllll}210 & 200 & 190 & 180 & 170 & 160 & 150 & 140 & 130 & 120 & 110 & 100 & 90 & 80 & 70 & 60 & 50 & 40 & 30 & 20 & 10 & 0\end{array}$ 


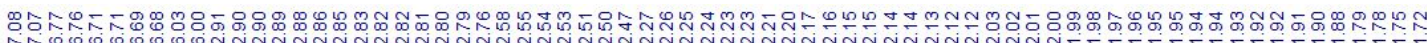<smiles>O=C1C=C[C@]2(CCCc3sccc32)CC1</smiles>

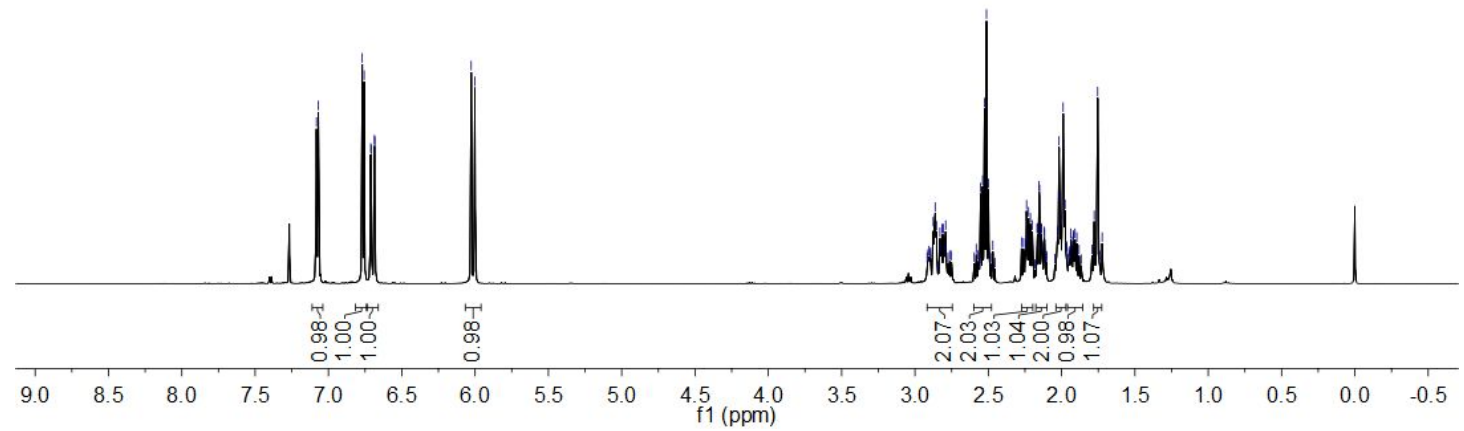<smiles>O=C1C=CC2(CCCc3sccc32)CC1</smiles>

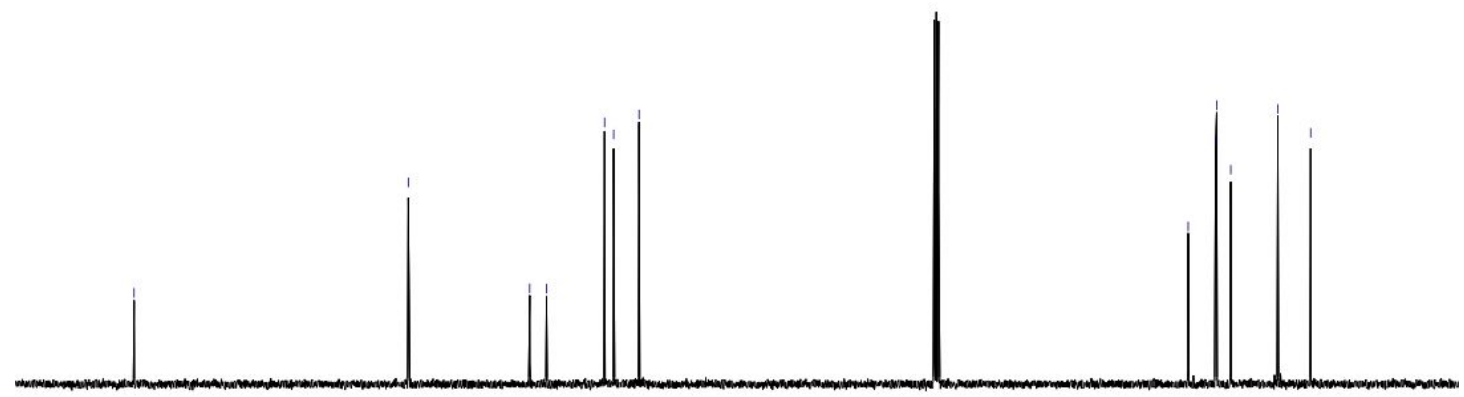

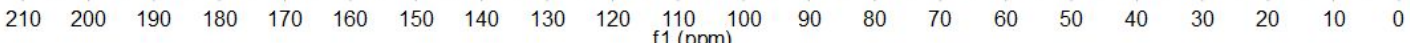


$4 j$

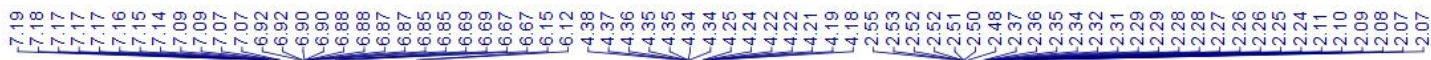<smiles>O=C1C=C[C@@]2(CCOc3ccccc32)CC1</smiles>

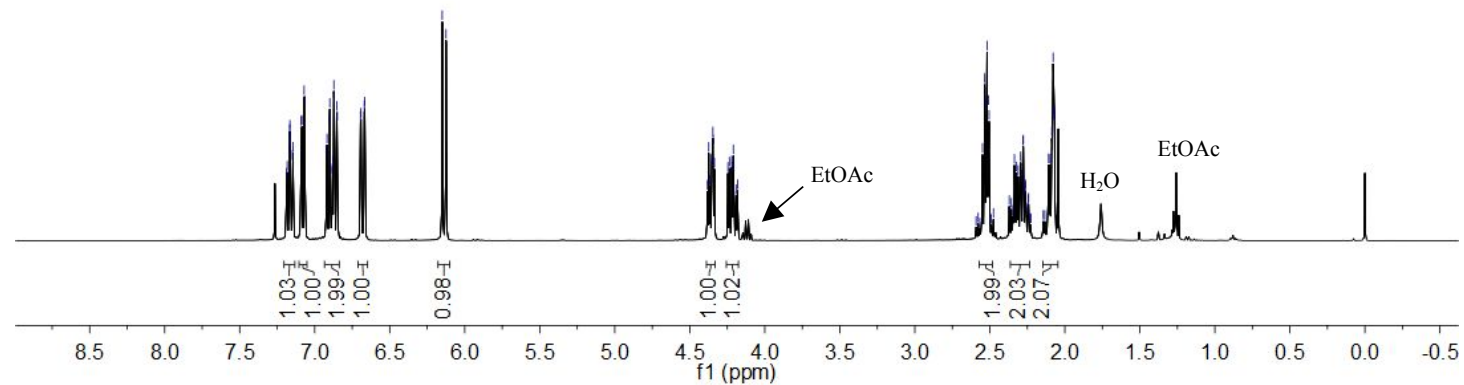<smiles>O=C1C=CC2(CCOc3ccccc32)CC1</smiles>

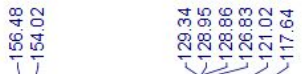

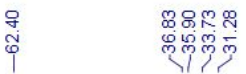

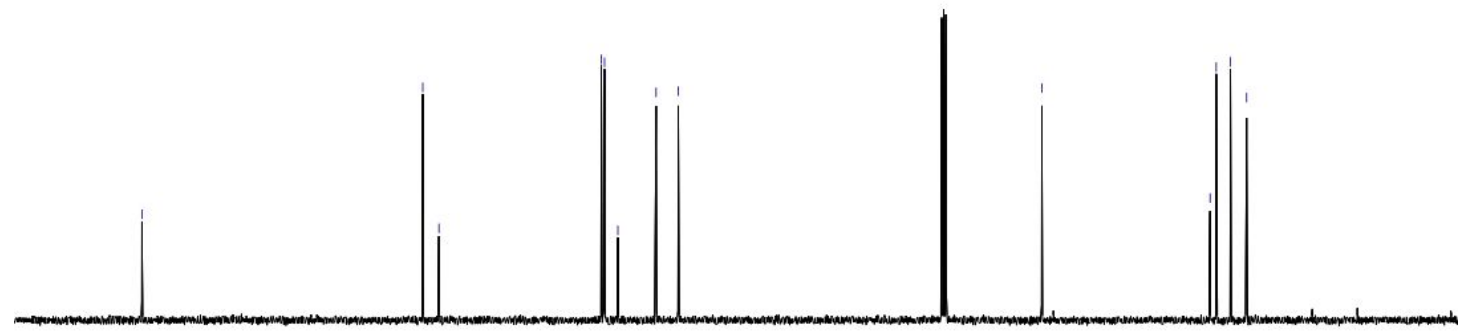

$\begin{array}{llllllllllllllllllllll}210 & 200 & 190 & 180 & 170 & 160 & 150 & 140 & 130 & 120 & \begin{array}{c}110 \\ \mathrm{f}(\mathrm{ppm})\end{array} & 100 & 90 & 80 & 70 & 60 & 50 & 40 & 30 & 20 & 10 & 0\end{array}$ 
<smiles>O=C1C=CC2(C=C1)CCSc1ccccc12</smiles>

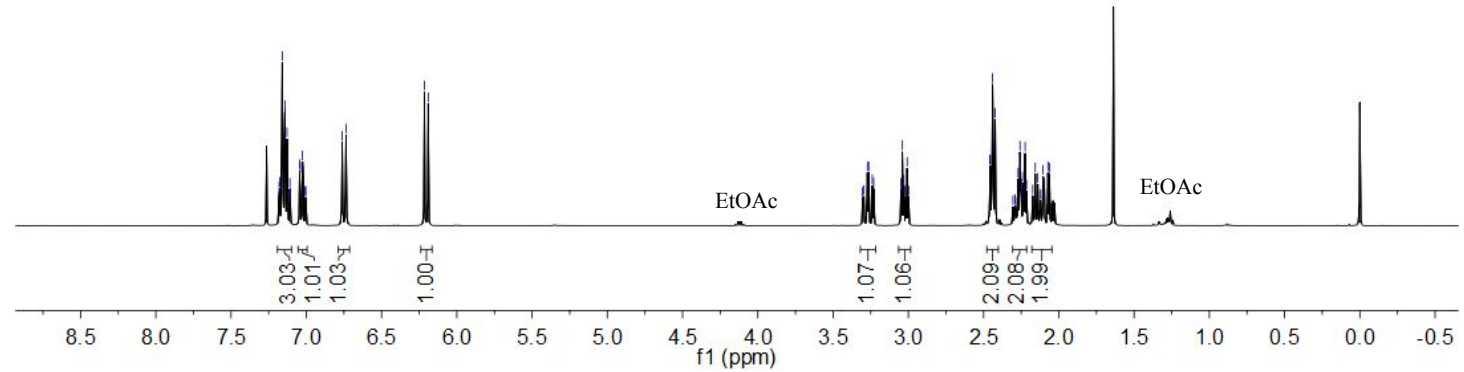<smiles>O=C1C=CC2(C=C1)CCSc1ccccc12</smiles>

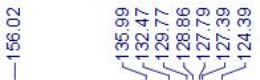

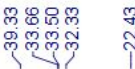

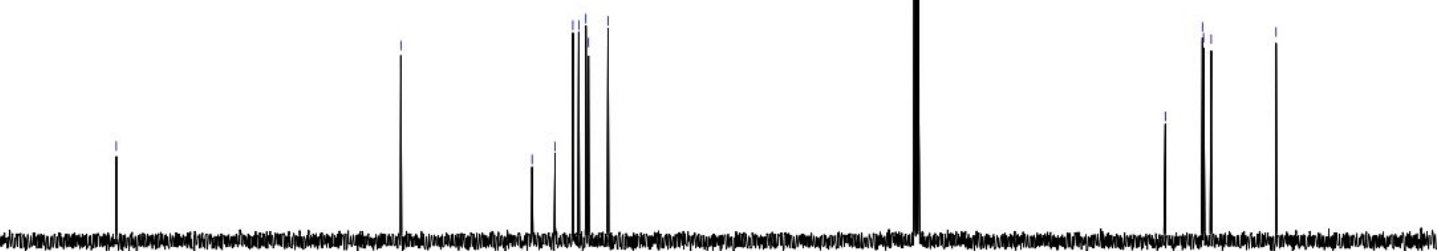

$\begin{array}{llllllllllllllllllllll}210 & 200 & 190 & 180 & 170 & 160 & 150 & 140 & 130 & 120 & \begin{array}{c}110 \\ \mathrm{f} 1(\mathrm{ppm})\end{array} & 100 & 90 & 80 & 70 & 60 & 50 & 40 & 30 & 20 & 10 & 0\end{array}$ 
41

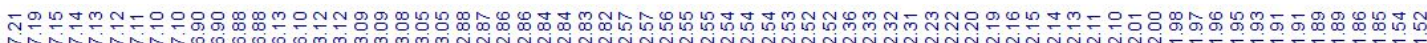<smiles>O=C1C=C[C@]2(CCCCc3ccccc32)CC1</smiles>

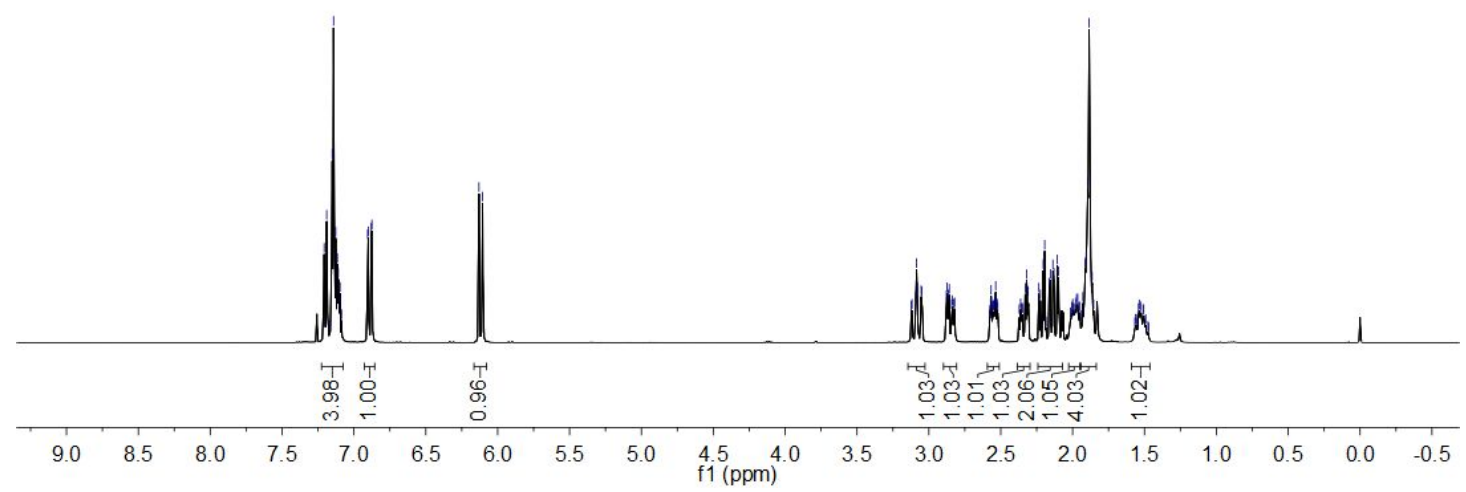

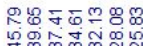<smiles>O=C1C=C[C@]2(CCCCc3ccccc32)CC1</smiles>

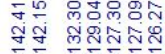

स्लmi

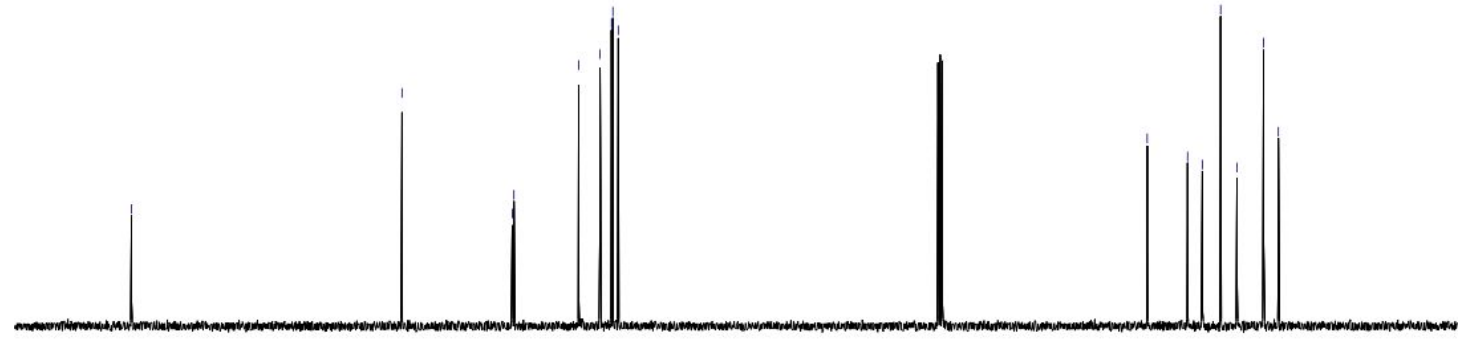

$\begin{array}{llllllllllllllllllllll}210 & 200 & 190 & 180 & 170 & 160 & 150 & 140 & 130 & 120 & \begin{array}{c}110 \\ 110\end{array} & 90 & 80 & 70 & 60 & 50 & 40 & 30 & 20 & 10 & 0\end{array}$ 


\section{HPLC chromatograms}<smiles>C[C@]1(c2ccccc2)C=CC(=O)CC1</smiles>

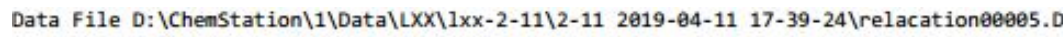
Sample Name: 160

\begin{tabular}{|c|c|c|c|}
\hline $\begin{array}{l}\text { Acq. Operator } \\
\text { Sample Operator }\end{array}$ & $\begin{array}{l}\text { : SYSTEM } \\
: \text { SYSTEM }\end{array}$ & Seq. Line : & 5 \\
\hline Acq. Instrument & : LC & Location : & P2-C. \\
\hline Injection Date & : 4/11/2019 6:50:44 PM & $\begin{array}{r}\text { Inj : } \\
\text { Inj Volume : }\end{array}$ & $\begin{array}{c}1 \\
2.909 \mu 1\end{array}$ \\
\hline
\end{tabular}

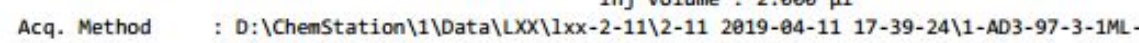
2UL-25C-25MIN.M

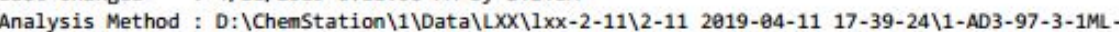
2UL-25C-25MIN.M (Sequence Method)

Last changed : 7/5/2019 9:53:33 AM by SYSTEM

(modified after loading)

Additional Info : Peak(s) manually integrated

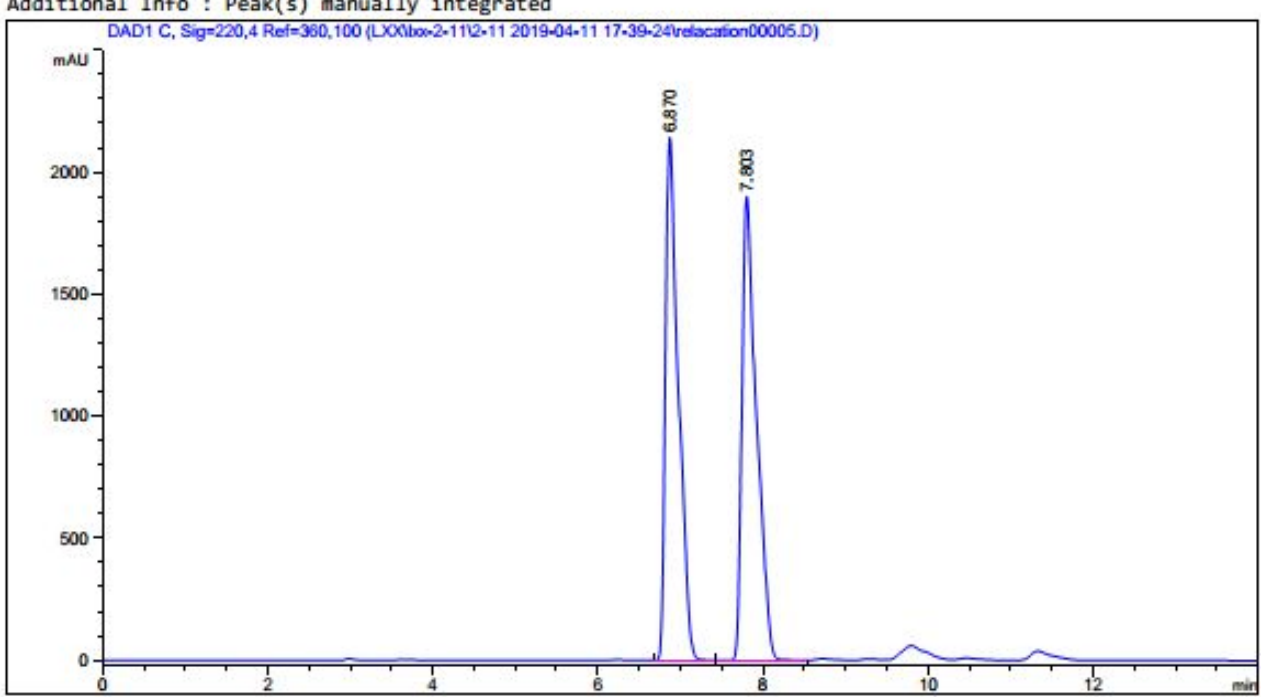

Area Percent Report

$\begin{array}{lll}\text { Sorted By } & : & \text { Signal } \\ \text { Multiplier } & : & 1.0000 \\ \text { Dilution } & : & 1.0000\end{array}$

Do not use Multiplier \& Dilution Factor with ISTDs

Signal 1: DAD1 C, Sig $=220,4$ Ref $=360,100$

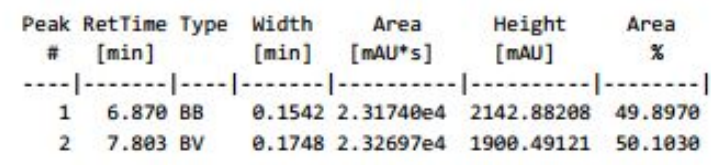

Totals :

$4.64437 \mathrm{e} 4 \quad 4643.37329$

LC 7/5/2019 9:53:45 AM SYSTEM 


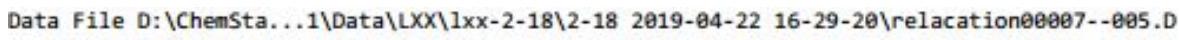
Sample Name: 18-2

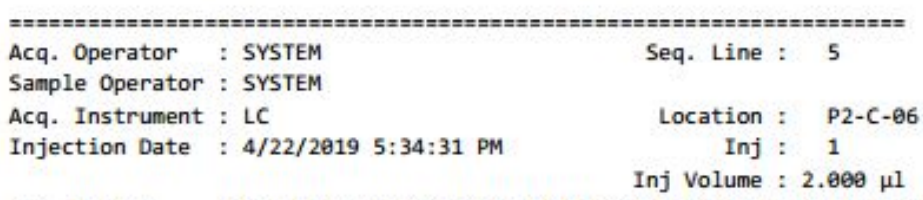

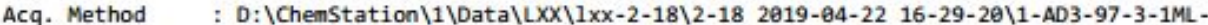
2UL-25C-15MIN.M

Last changed : 11/28/2018 10:54:45 AM by SYSTEM

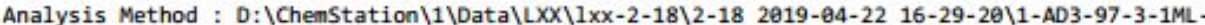
2UL-25C-15MIN.M (Sequence Method)

Last changed : 7/5/2019 1:41:06 PM by SYSTEM (modified after loading)

Additional Info : Peak(s) manually integrated

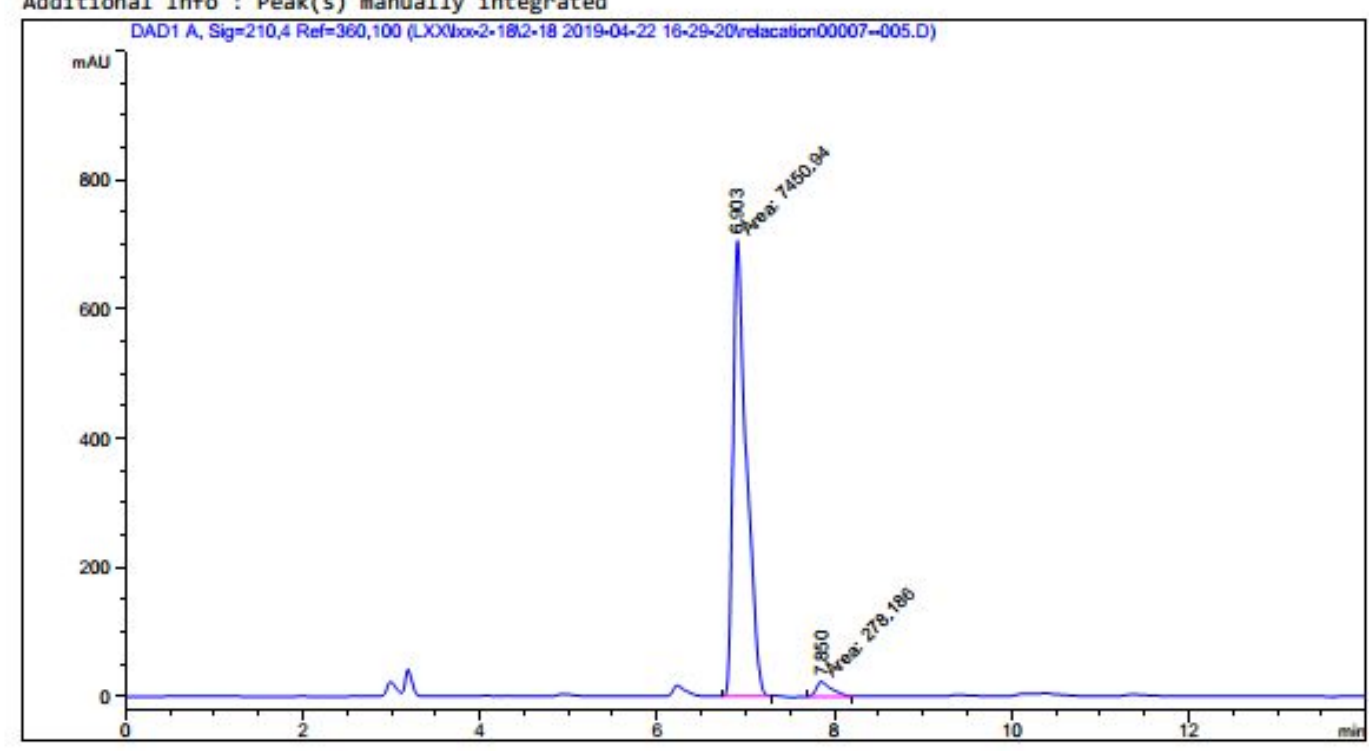

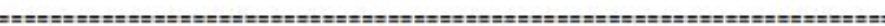

Area Percent Report

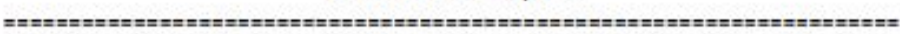

$\begin{array}{lll}\text { Sorted By } & : & \text { Signal } \\ \text { Multiplier } & : & 1.0000 \\ \text { Dilution } & : & 1.0000\end{array}$

Do not use Multiplier \& Dilution Factor with ISTDs

Signal 1: DAD1 A, Sig=210,4 Ref=360, 100

\begin{tabular}{cccccc}
$\begin{array}{c}\text { Peak RetTime Type } \\
\text { [min] }\end{array}$ & $\begin{array}{c}\text { Width } \\
\text { [min] }\end{array}$ & $\begin{array}{c}\text { Area } \\
\text { [mAU*s] }\end{array}$ & $\begin{array}{l}\text { Height } \\
\text { [mAU] }\end{array}$ & $\begin{array}{c}\text { Area } \\
\%\end{array}$ \\
\hline 1 & $6.903 \mathrm{Mm}$ & 0.1756 & 7450.93750 & 707.06372 & 96.4008 \\
2 & $7.850 \mathrm{MM}$ & 0.1989 & 278.18594 & 23.30510 & 3.5992 \\
& & & & & \\
Totals : & & 7729.12344 & 730.36882
\end{tabular}


<smiles>Cc1ccc([C@]2(C)C=CC(=O)CC2)cc1</smiles>

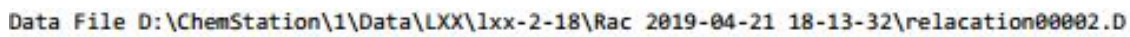
Sample Name: p-Me-Rac

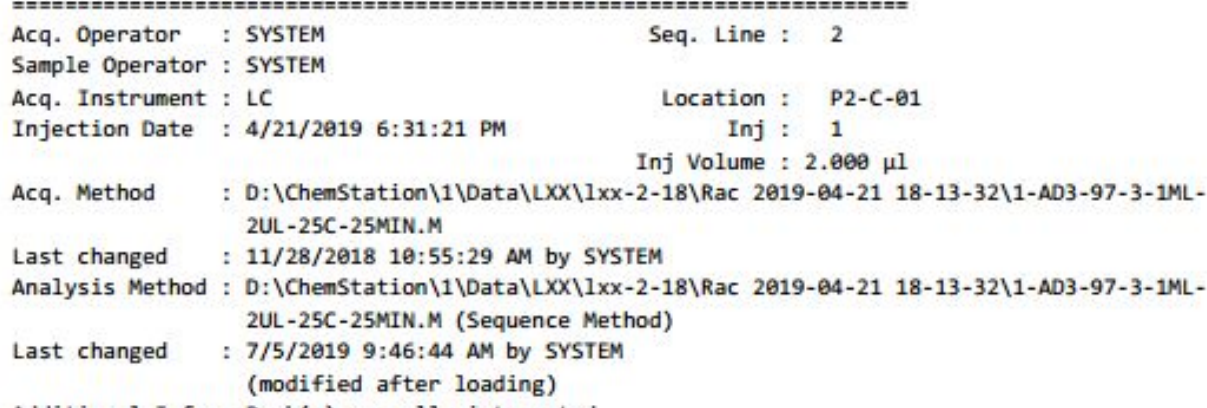

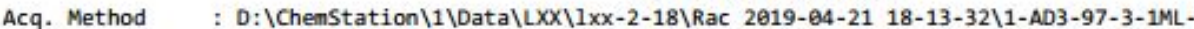
$2 \mathrm{UL}-25 \mathrm{C}-25 \mathrm{MIN} . \mathrm{M}$

Last changed : 11/28/2018 10:55:29 AM by SYSTEM

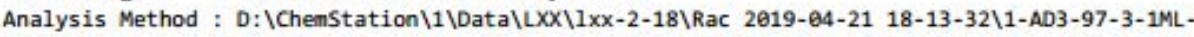

2UL-25C-25MIN.M (Sequence Method)

Last changed : 7/5/2019 9:46:44 AM by SYSTEM

(modified after loading)

Additional Info: Peak(s) manually integrated

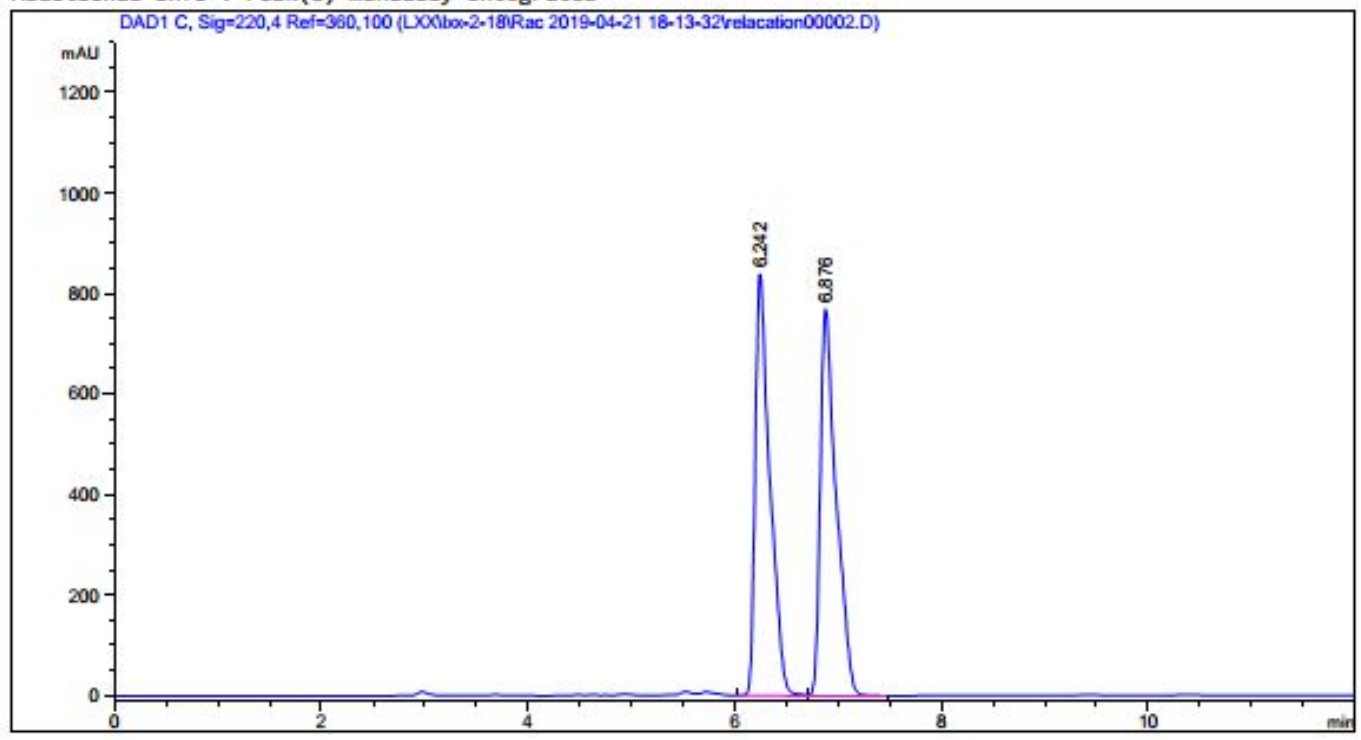

Area Percent Report

$\begin{array}{lll} & & \text { Area PerCent Report } \\ & & \\ \text { Sorted By } & : & \text { Signal } \\ \text { Multiplier } & : & 1.000 \text { } \\ \text { Dilution } & : & 1.000 \text { S }\end{array}$

Do not use Multiplier \& Dilution Factor with ISTDs

Signal 1: DAD1 C, $S i g=220,4$ Ref $=360,100$

\begin{tabular}{cccccc}
$\begin{array}{c}\text { Peak RetTime Type } \\
\text { [min] }\end{array}$ & $\begin{array}{c}\text { Width } \\
\text { [min] }\end{array}$ & $\begin{array}{c}\text { Area } \\
\text { [mAU*s] }\end{array}$ & $\begin{array}{c}\text { Height } \\
\text { [mAU] }\end{array}$ & $\begin{array}{c}\text { Area } \\
\%\end{array}$ \\
\hline 1 & 6.242 BV & 0.1407 & 8258.53223 & 840.08234 & 50.0534 \\
2 & 6.876 VB & 0.1527 & 8240.92480 & 770.70258 & 49.9466 \\
& & & & & \\
Totals : & & $1.64995 e 4$ & 1610.78491
\end{tabular}




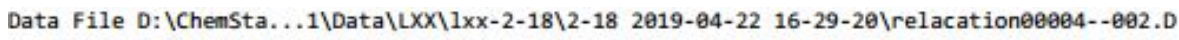
Sample Name: 18-6

\begin{tabular}{|c|c|c|c|}
\hline $\begin{array}{l}\text { Acq. Operator } \\
\text { Sample Operator }\end{array}$ & $\begin{array}{l}\text { : SYSTEM } \\
\text { : SYSTEM }\end{array}$ & Seq. Line & : \\
\hline Acq. Instrument & : LC & Location & $\mathrm{P} 2-\mathrm{C}-03$ \\
\hline Injection Date & : 4/22/2019 4:47:02 PM & $\begin{array}{r}\text { Inj } \\
\text { Inj Volume }\end{array}$ & $\begin{array}{l}: \quad 1 \\
: 2.000 \mu 1\end{array}$ \\
\hline
\end{tabular}

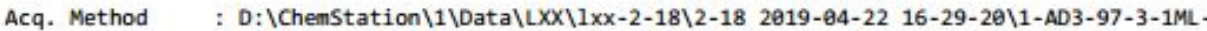
2UL - 25C-15MIN.M

Last changed : 11/28/2018 10:54:45 AM by SYSTEM

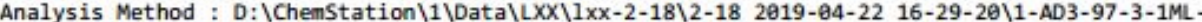
2UL-25C-15MIN.M (Sequence Method)

Last changed : 7/5/2019 9:39:01 AM by SYSTEM

(modified after loading)

Additional Info: Peak(s) manually integrated

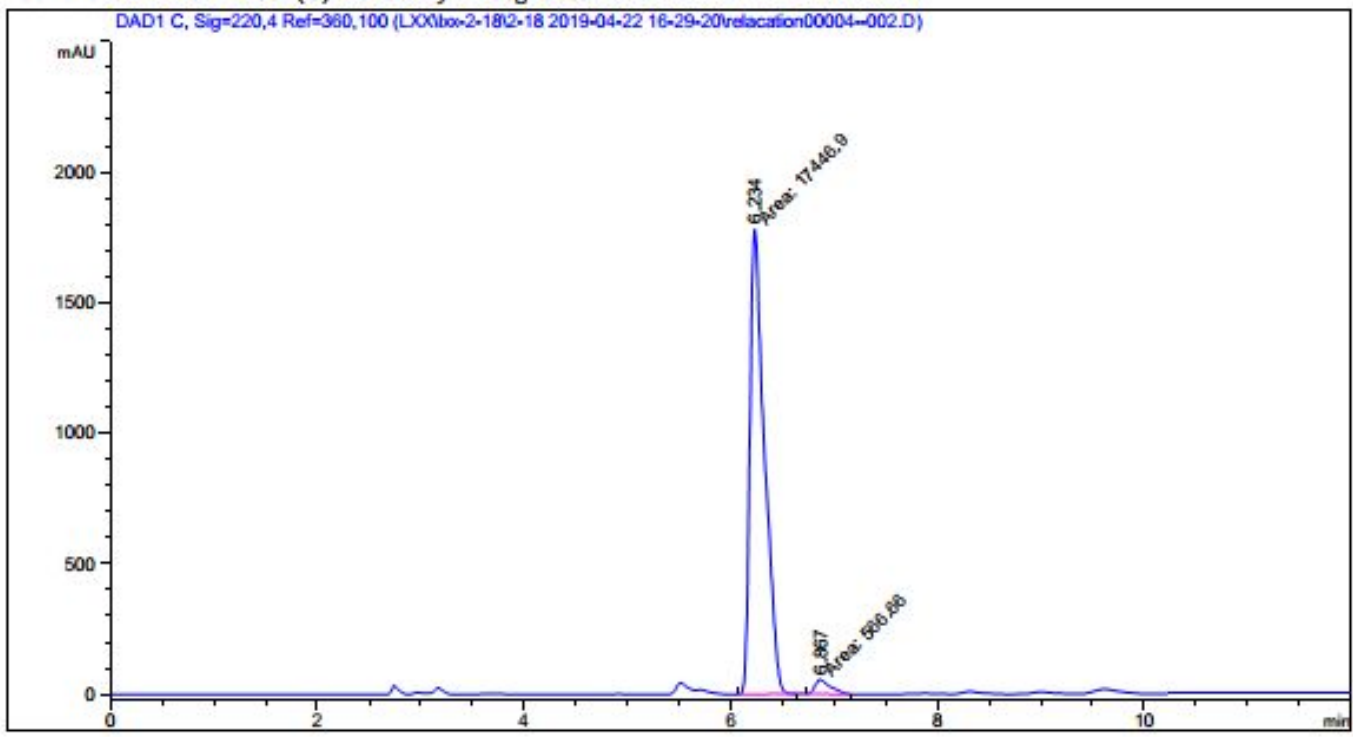

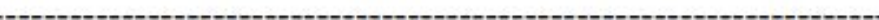

Area Percent Report

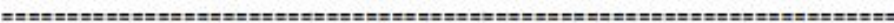

$\begin{array}{lll}\text { Sorted By } & : & \text { Signal } \\ \text { Multiplier } & : & 1.0000 \\ \text { Dilution } & : & 1.0000\end{array}$

Do not use Multiplier \& Dilution Factor with ISTDs

Signal 1: DAD1 C, Sig $=220,4$ Ref $=360,100$

\begin{tabular}{|c|c|c|c|c|c|c|}
\hline $\begin{array}{c}\text { Peak } \\
\#\end{array}$ & $\begin{array}{l}\text { RetTime } \\
\text { [min] }\end{array}$ & Type & $\begin{array}{l}\text { Width } \\
\text { [min] }\end{array}$ & $\begin{array}{c}\text { Area } \\
{\left[\mathrm{mAU}^{*} \mathrm{~s}\right]}\end{array}$ & $\begin{array}{l}\text { Height } \\
\text { [MAU] }\end{array}$ & $\begin{array}{c}\text { Area } \\
\%\end{array}$ \\
\hline$\cdots-1$ & 1 & & & |-n. & |1-n. & -........ \\
\hline 1 & 6.234 & $\mathrm{MM}$ & 0.1639 & $1.74469 \mathrm{e} 4$ & 1783.84473 & 96.8543 \\
\hline 2 & 6.867 & $\mathrm{MM}$ & 0.1711 & 566.66028 & 55.18898 & 3.1457 \\
\hline Tot & & & & $1.80135 \mathrm{e} 4$ & 1839.03371 & \\
\hline
\end{tabular}

LC 7/5/2019 9:39:10 AM SYSTEM 
<smiles>COc1ccc([C@]2(C)C=CC(=O)CC2)cc1</smiles>

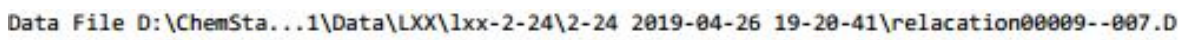
Sample Name: p-OMe-Rac

\begin{tabular}{|c|c|c|c|}
\hline $\begin{array}{l}\text { Acq. Operator } \\
\text { Sample Operator }\end{array}$ & $\begin{array}{l}: \text { SYSTEM } \\
: \text { SYSTEM }\end{array}$ & Seq. Line : & 7 \\
\hline Acq. Instrument & : LC & Location : & P2-C-67 \\
\hline njection Date & : 4/26/2019 8:44:26 PM & $\begin{array}{r}\text { Inj : } \\
\text { Inj Volume : }\end{array}$ & $\begin{array}{l}: \quad 1 \\
: 2.009 \mu \mathrm{l}\end{array}$ \\
\hline
\end{tabular}

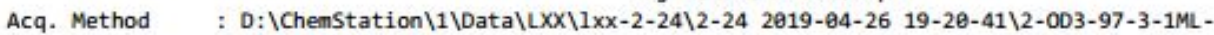
2UL - 25C-15MIN.M

Last changed : 11/14/2018 6:31:09 PM by SYSTEM

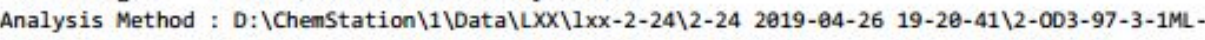

2UL-25C-15MIN.M (Sequence Method)

Last changed : 7/5/2019 10:14:11 AM by SYSTEM

(modified after loading)

Additional Info: Peak(s) manually integrated

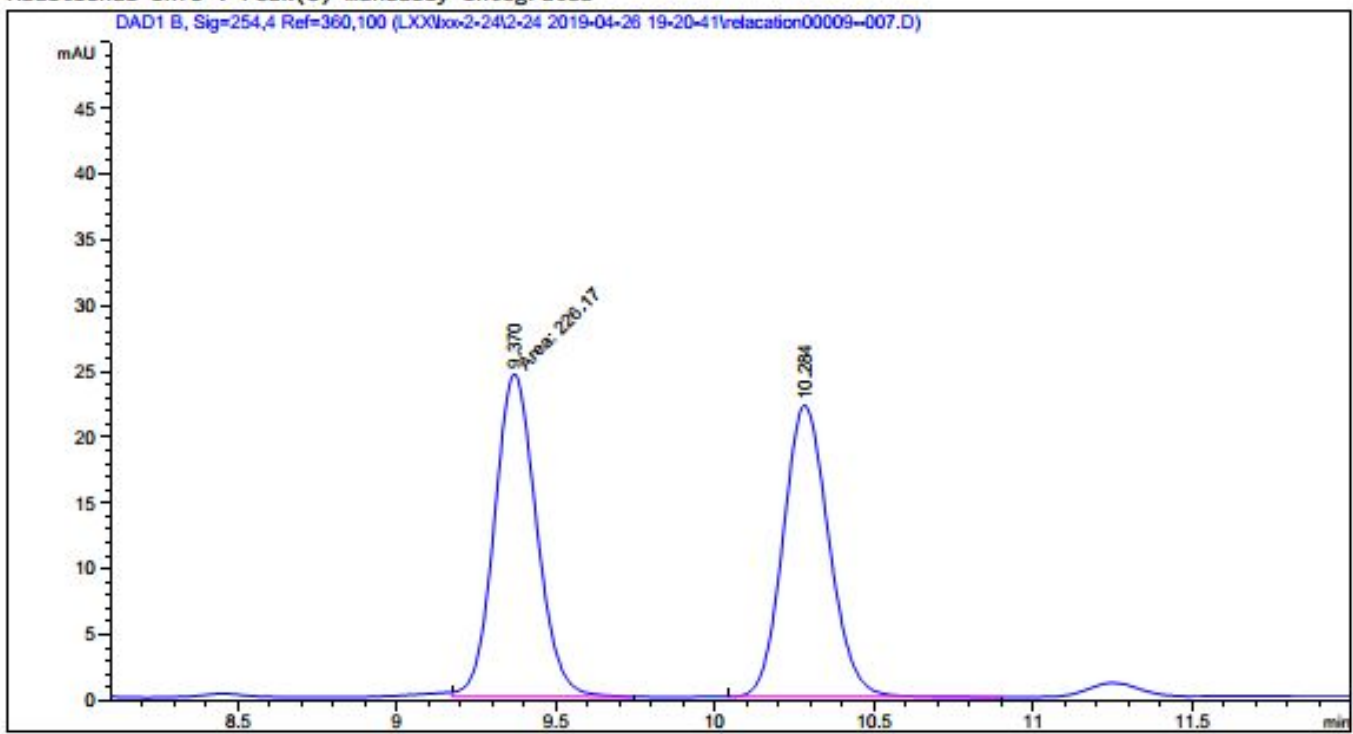

Area Percent Report

\begin{tabular}{|c|c|c|c|c|}
\hline Sorted By & : & \multicolumn{3}{|l|}{ Signal } \\
\hline Multiplier & : & \multicolumn{3}{|l|}{1.0000} \\
\hline Dilution & : & \multicolumn{3}{|l|}{$1.000 \theta$} \\
\hline \multicolumn{5}{|c|}{ Do not use Multiplier \& Dilution Factor with ISTDs } \\
\hline \multicolumn{5}{|c|}{ Signal 1: DAD1 B, Sig=254,4 Ref $=360,100$} \\
\hline $\begin{array}{l}\text { Peak RetTime Type } \\
\# \quad[\mathrm{~min}]\end{array}$ & $\begin{array}{l}\text { Width } \\
\text { [min] }\end{array}$ & $\begin{array}{c}\text { Area } \\
{\left[\mathrm{mAU}^{*} \mathrm{~s}\right]}\end{array}$ & $\begin{array}{l}\text { Height } \\
\text { [mAU] }\end{array}$ & $\begin{array}{c}\text { Area } \\
\quad \%\end{array}$ \\
\hline 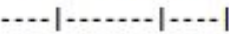 & 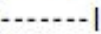 & 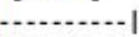 & 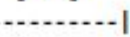 & $-\ldots-1$ \\
\hline $19.370 \mathrm{~mm}$ & 0.1536 & 226.17038 & 24.53386 & 50.5947 \\
\hline $210.284 \mathrm{BB}$ & 0.1543 & 220.85384 & 22.15163 & 49.4953 \\
\hline Totals : & & 447.02342 & 46.68549 & \\
\hline
\end{tabular}

LC 7/5/2019 10:14:14 AM SYSTEM 


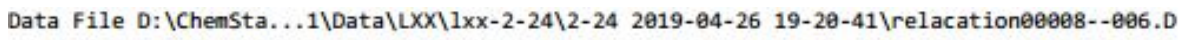
Sample Name: 24-6

\begin{tabular}{|c|c|c|c|}
\hline $\begin{array}{l}\text { Acq. Operator } \\
\text { Sample Operator }\end{array}$ & $\begin{array}{l}\text { : SYSTEM } \\
: \text { SYSTEM }\end{array}$ & Seq. Line & 6 \\
\hline Acq. Instrument & : LC & Location & P2-C-06 \\
\hline Injection Date & : 4/26/2019 8:28:36 PM & $\begin{array}{r}\text { Inj } \\
\text { Inj Volume }\end{array}$ & $\begin{array}{l}: \quad 1 \\
: 2.000 \mu 1\end{array}$ \\
\hline
\end{tabular}

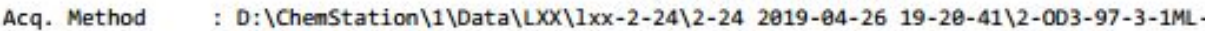
2UL-25C-15MIN. M

Last changed : 11/14/2018 6:31:e9 PM by SYSTEM

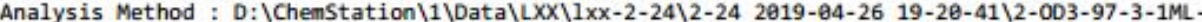
2UL-25C-15MIN.M (Sequence Method)

Last changed : 7/5/2019 10:15:46 AM by SYSTEM

(modified after loading)

Additional Info: Peak(s) manually integrated

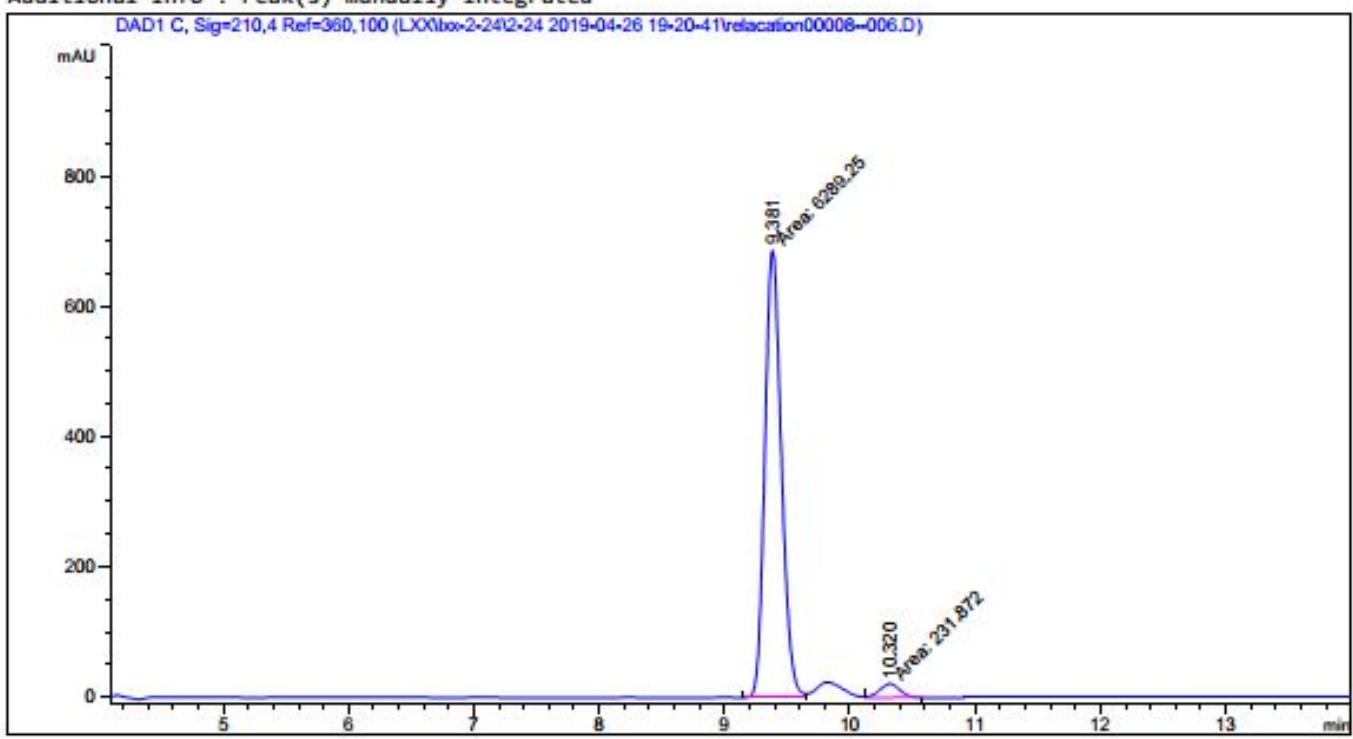

(1)

Area Percent Report

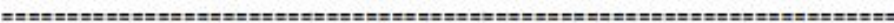

$\begin{array}{lll}\text { Sorted By } & : & \text { Signal } \\ \text { Multiplier } & : & 1.0000 \\ \text { Dilution } & : & 1.0000\end{array}$

Do not use Multiplier \& Dilution Factor with ISTDs

Signal 1: DAD1 C, Sig=210,4 Ref=360, 100

\begin{tabular}{|c|c|c|c|c|c|c|}
\hline $\begin{array}{c}\text { Peak } \\
\#\end{array}$ & $\begin{array}{l}\text { RetTime } \\
\text { [min] }\end{array}$ & Type & $\begin{array}{l}\text { Width } \\
\text { [min] }\end{array}$ & $\begin{array}{c}\text { Area } \\
{\left[\mathrm{mAU}^{*} \mathrm{~s}\right]}\end{array}$ & $\begin{array}{l}\text { Height } \\
\text { [mAU] }\end{array}$ & $\begin{array}{c}\text { Area } \\
\%\end{array}$ \\
\hline$\ldots-1$ & $\cdots$ & & (n) & |-...... & | & | \\
\hline 1 & 9.381 & MF & 0.1525 & 6289.24756 & 687.39028 & 96.4443 \\
\hline 2 & 10.320 & FM & 0.1833 & 231.87163 & 21.08619 & 3.5557 \\
\hline otal & : & & & 6521.11919 & 798.47638 & \\
\hline
\end{tabular}

LC 7/5/2019 10:15:50 AM SYSTEM 
<smiles>C[C@]1(c2ccc(C(F)(F)F)cc2)C=CC(=O)CC1</smiles>

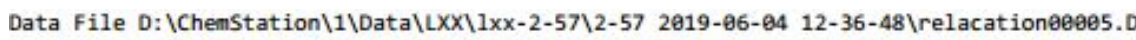
Sample Name: 57-5-RAC

\begin{tabular}{|c|c|c|c|}
\hline $\begin{array}{l}\text { Acq. Operator } \\
\text { Sample Operator }\end{array}$ & $\begin{array}{l}\text { : SYSTEM } \\
: \text { SYSTEM }\end{array}$ & Seq. Line & 5 \\
\hline Acq. Instrument & : LC & Location & P2-C-65 \\
\hline njection Date & : 6/4/2019 1:41:15 PM & $\begin{array}{r}\text { Inj } \\
\text { Inj Volume }\end{array}$ & $\begin{array}{l}: \quad 1 \\
: 2.000 \mu \mathrm{l}\end{array}$ \\
\hline
\end{tabular}

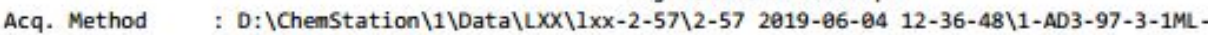
2UL-25C-15MIN. M

Last changed : 11/28/2018 10:54:45 AM by SYSTEM

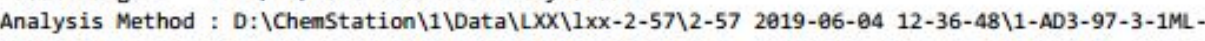

2UL-25C-15MIN.M (Sequence Method)

Last changed : 7/5/2019 1:58:06 PM by SYSTEM

(modified after loading)

Additional Info: Peak(s) manually integrated
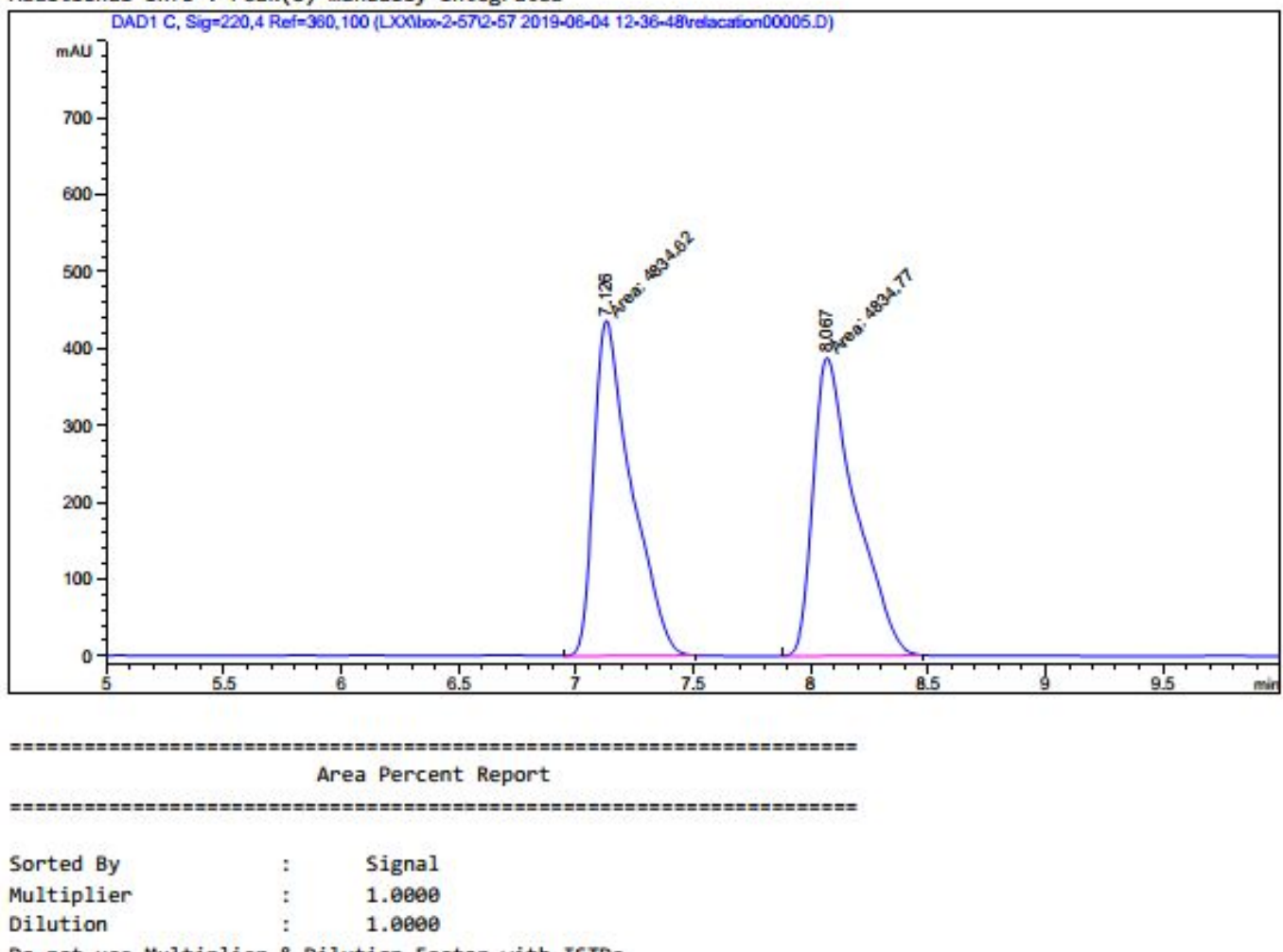

Do not use Multiplier \& Dilution Factor with ISTDs

Signal 1: DAD1 C, $S i g=220,4$ Ref $=360,100$

\begin{tabular}{cccccc}
$\begin{array}{c}\text { Peak RetTime Type } \\
\text { \# } \\
\text { [min] }\end{array}$ & $\begin{array}{c}\text { Width } \\
\text { [min] }\end{array}$ & $\begin{array}{c}\text { Area } \\
\text { [mAU*s] }\end{array}$ & $\begin{array}{c}\text { Height } \\
\text { [mAU] }\end{array}$ & $\begin{array}{c}\text { Area } \\
\%\end{array}$ \\
\hline 1 & $7.126 \mathrm{MM}$ & 0.1848 & 4834.62297 & 435.99911 & 49.9992 \\
2 & $8.867 \mathrm{MM}$ & 0.2079 & 4834.77441 & 387.65765 & 50.0008
\end{tabular}




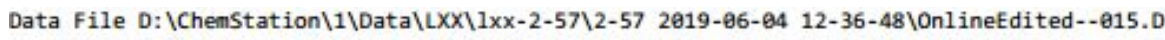
Sample Name: 57-5

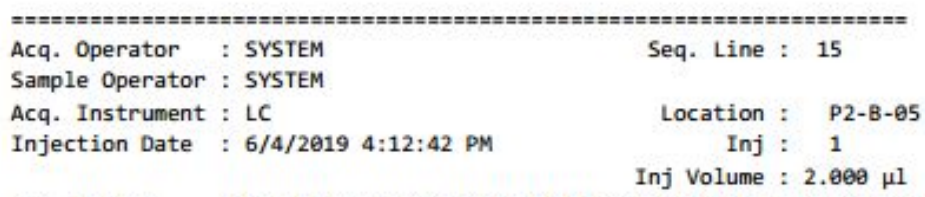

Acq. Method : D:\ChemStation\1\Data\LXX\1XX-2-57\2-57 2019-06-04 12-36-48\1-AD3-97-3-1ML2UL-25C-15MIN.M

Last changed : 6/4/2019 4:09:42 PM by SYSTEM

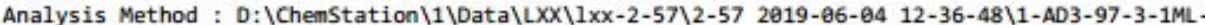
2UL-25C-15MIN.M (Sequence Method)

Last changed : 7/5/2019 1:55:56 PM by SYSTEM (modified after loading)

Additional Info : Peak(s) manually integrated

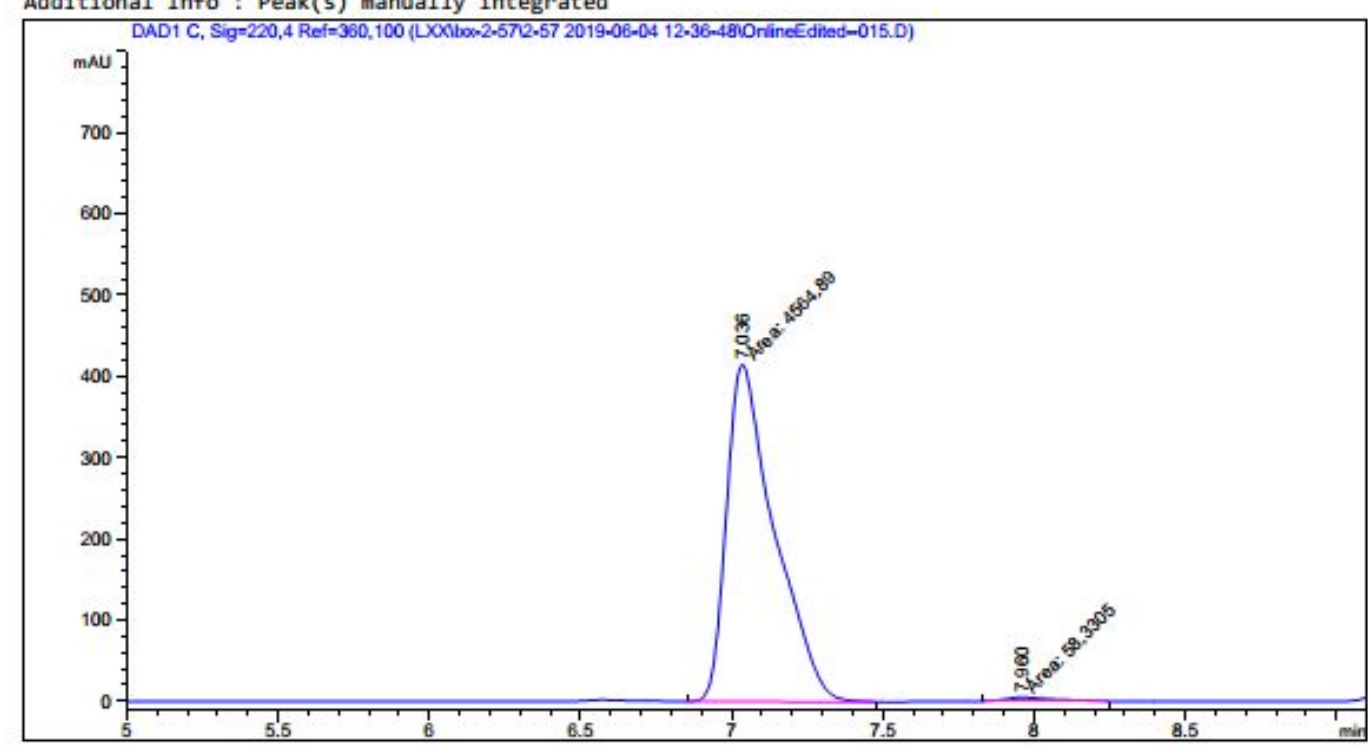

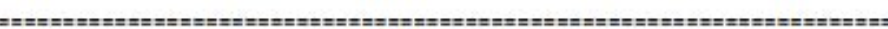

Area Percent Report

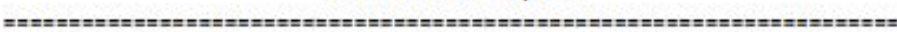

$\begin{array}{lll}\text { Sorted By } & : & \text { Signal } \\ \text { Multiplier } & : & 1.0000 \\ \text { Dilution } & : & 1.0000\end{array}$

Do not use Multiplier \& Dilution Factor with ISTDs

Signal 1: DAD1 C, Sig=220,4 Ref=360, 100

\begin{tabular}{|c|c|c|c|c|c|c|}
\hline $\begin{array}{c}\text { Peak } \\
\#\end{array}$ & $\begin{array}{l}\text { RetTime } \\
\text { [min] }\end{array}$ & Type & $\begin{array}{l}\text { Width } \\
\text { [min] }\end{array}$ & $\begin{array}{c}\text { Area } \\
{\left[\mathrm{mAU}^{*} \mathrm{~s}\right]}\end{array}$ & $\begin{array}{l}\text { Height } \\
\text { [mAU] }\end{array}$ & $\begin{array}{c}\text { Area } \\
\%\end{array}$ \\
\hline$\ldots-1$ & | & $1 \cdots$ & - & | & - & -........ \\
\hline 1 & 7.036 & MM & 0.1832 & 4564.88770 & 415.25702 & 98.7383 \\
\hline 2 & 7.968 & MM & 0.2001 & 58.33649 & 4.85937 & 1.2617 \\
\hline Total & 1s: & & & 4623.21819 & 420.11639 & \\
\hline
\end{tabular}


<smiles>CC1(c2ccc(F)cc2)C=CC(=O)CC1</smiles>

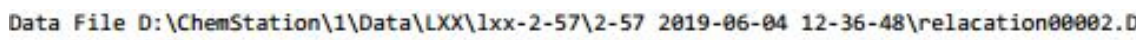
Sample Name: 57-2-RAC

\begin{tabular}{|c|c|c|c|}
\hline $\begin{array}{l}\text { Acq. Operator } \\
\text { Sample Operator }\end{array}$ & $\begin{array}{l}\text { : SYSTEM } \\
: \text { SYSTEM }\end{array}$ & Seq. Line & 2 \\
\hline Acq. Instrument & : LC & Location & $\mathrm{P} 2-\mathrm{C}-62$ \\
\hline Injection Date & : 6/4/2019 12:53:47 PM & $\begin{array}{r}\text { Inj } \\
\text { Inj Volume }\end{array}$ & $\begin{array}{l}: \quad 1 \\
: 2.000 \mu \mathrm{l}\end{array}$ \\
\hline
\end{tabular}

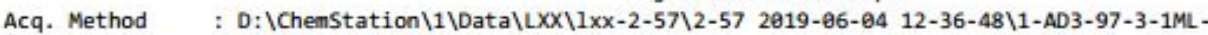
2UL-25C-15MIN. M

Last changed : 11/28/2018 10:54:45 AM by SYSTEM

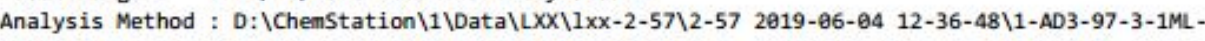

2UL-25C-15MIN.M (Sequence Method)

Last changed : 7/5/2019 1:47:49 PM by SYSTEM

(modified after loading)

Additional Info: Peak(s) manually integrated

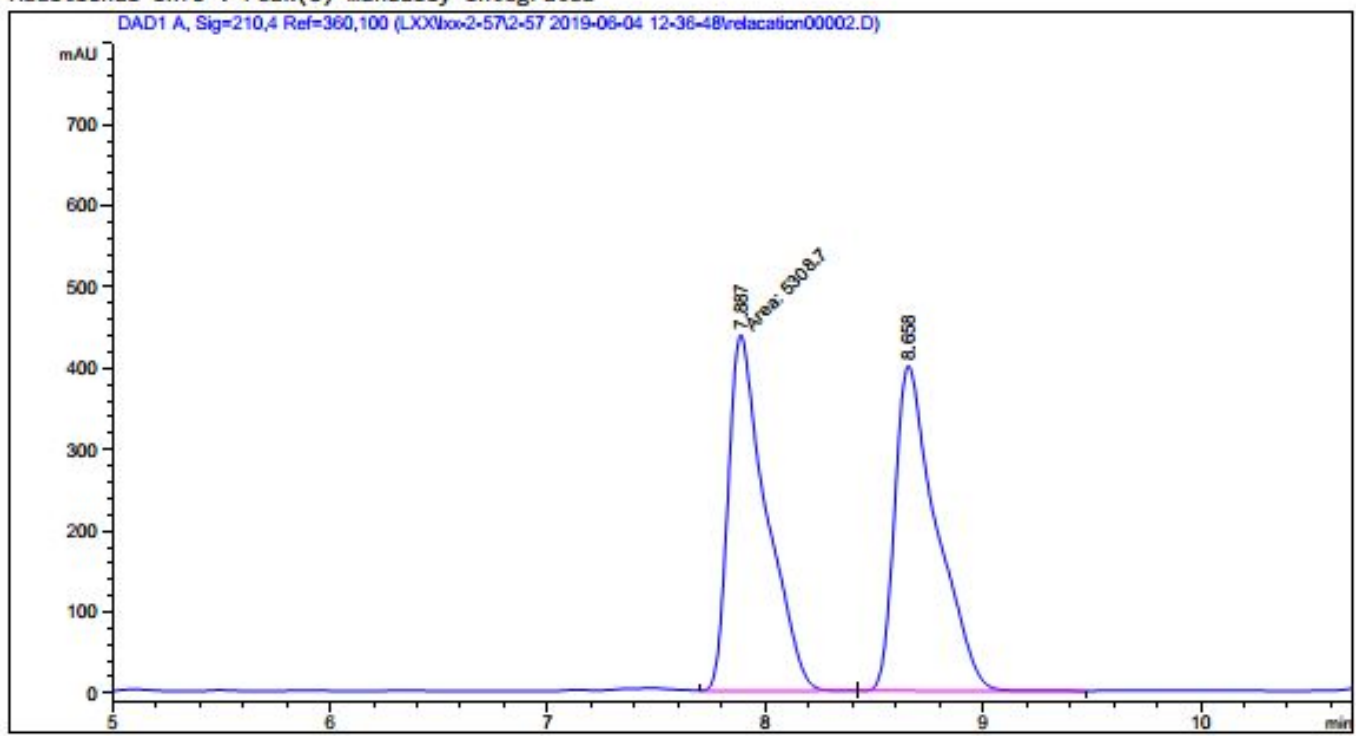

Area Percent Report

$\begin{array}{lll} & & \text { Area PerCent Report } \\ & & \\ \text { Sorted By } & : & \text { Signal } \\ \text { Multiplier } & : & 1.000 \text { } \\ \text { Dilution } & : & 1.000 \text { S }\end{array}$

Do not use Multiplier \& Dilution Factor with ISTDs

Signal 1: DAD1 A, $S i g=210,4$ Ref $=360,100$

\begin{tabular}{|c|c|c|c|c|c|c|}
\hline $\begin{array}{c}\text { Peak } \\
\#\end{array}$ & $\begin{array}{l}\text { RetTime } \\
\text { [min] }\end{array}$ & Type & $\begin{array}{l}\text { Width } \\
\text { [min] }\end{array}$ & $\begin{array}{c}\text { Area } \\
{\left[\mathrm{mAU}^{*} \mathrm{~s}\right]}\end{array}$ & $\begin{array}{l}\text { Height } \\
\text { [mAU] }\end{array}$ & $\begin{array}{c}\text { Area } \\
\%\end{array}$ \\
\hline$\ldots$ & $|\ldots|$ & 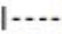 & .......... & | & . & - \\
\hline 1 & 7.887 & FM & 0.2017 & 5308.70068 & 438.61310 & 49.8877 \\
\hline 2 & 8.658 & BB & 0.1893 & 5332.60889 & 400.48596 & 50.1123 \\
\hline Total & $15:$ & & & $1.06413 \mathrm{e} 4$ & 839.09906 & \\
\hline
\end{tabular}




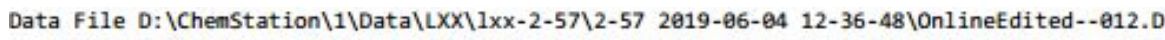
Sample Name: 57-2

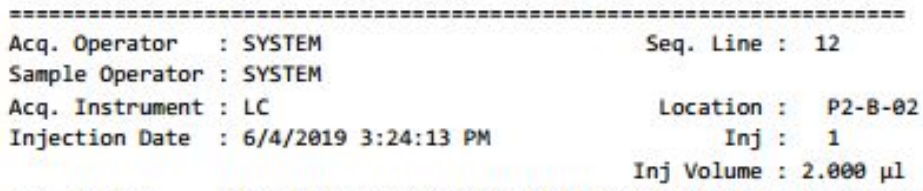

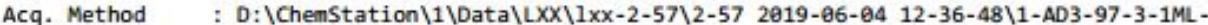
2UL-25C-15MIN. M

Last changed : 11/28/2018 10:54:45 AM by SYSTEM

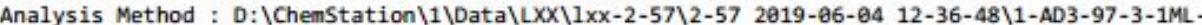
2UL-25C-15MIN.M (Sequence Method)

Last changed : 7/5/2019 1:46:40 PM by SYSTEM (modified after loading)

Additional Info: Peak(s) manually integrated
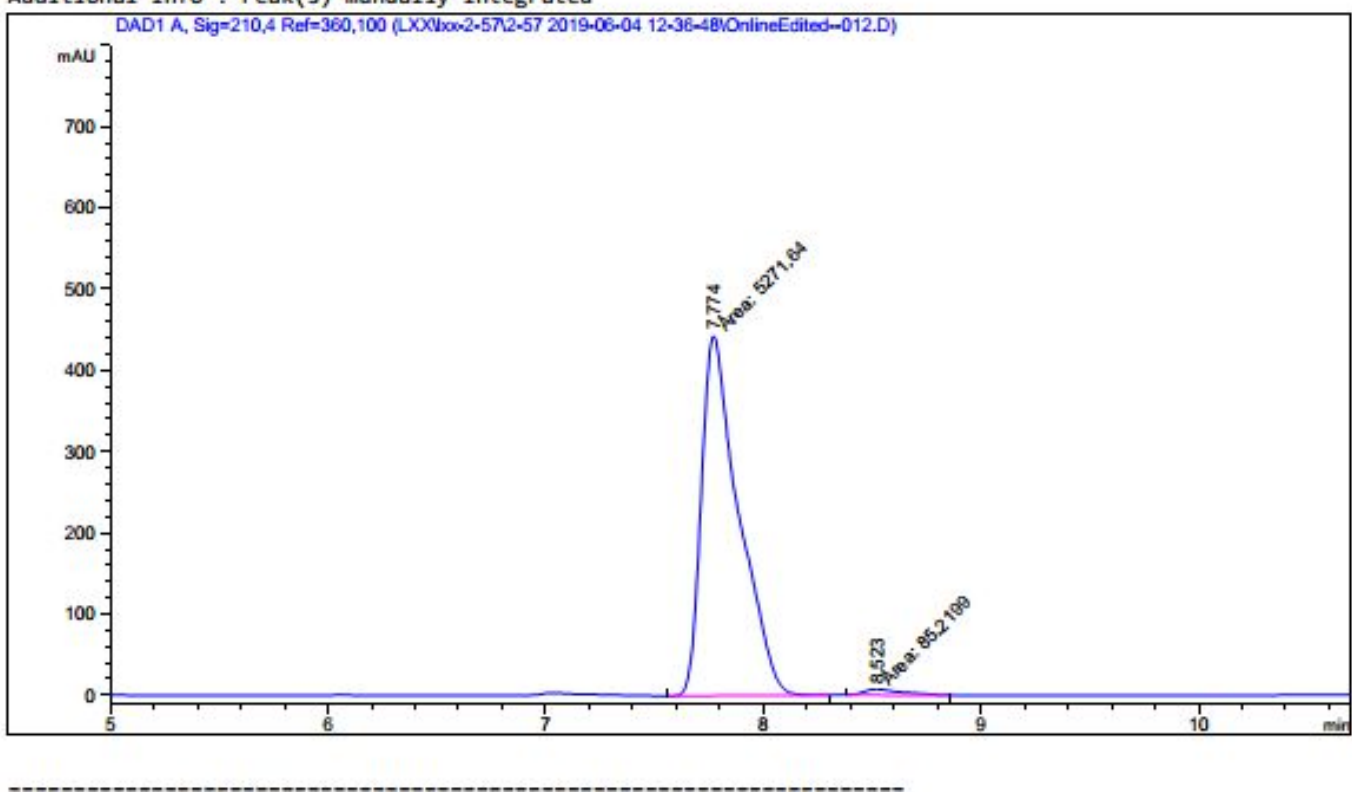

Area Percent Report

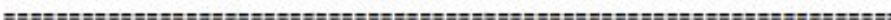

$\begin{array}{lll}\text { Sorted By } & : & \text { Signal } \\ \text { Multiplier } & : & 1.0000 \\ \text { Dilution } & : & 1.0000\end{array}$

Do not use Multiplier \& Dilution Factor with ISTDs

Signal 1: DAD1 A, Sig=210,4 Ref=360, 100

\begin{tabular}{|c|c|c|c|c|c|c|}
\hline $\begin{array}{c}\text { Peak } \\
\#\end{array}$ & $\begin{array}{c}\text { RetTime } \\
\text { [min] }\end{array}$ & Type & $\begin{array}{l}\text { Width } \\
\text { [min] }\end{array}$ & $\begin{array}{c}\text { Area } \\
{\left[\mathrm{mAU}^{*} \mathrm{~s}\right]}\end{array}$ & $\begin{array}{l}\text { Height } \\
\text { [MAU] }\end{array}$ & $\begin{array}{c}\text { Area } \\
\%\end{array}$ \\
\hline 1 & & & & - & . & -....... \\
\hline 1 & 7.774 & $\mathrm{MM}$ & 0.1984 & 5271.63916 & 442.88800 & 98.4091 \\
\hline 2 & 8.523 & $\mathrm{MM}$ & 0.2049 & 85.21989 & 6.93059 & 1.5999 \\
\hline $\mathrm{T}$ & & & & 5356.85905 & 449.81859 & \\
\hline
\end{tabular}


<smiles>CC1(c2ccc(Cl)cc2)C=CC(=O)CC1</smiles>

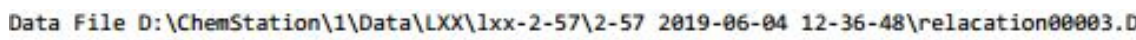
Sample Name: 57-3-RAC

\begin{tabular}{|c|c|c|c|}
\hline $\begin{array}{l}\text { Acq. Operator } \\
\text { Sample Operator }\end{array}$ & $\begin{array}{l}: \text { SYSTEM } \\
: \text { SYSTEM }\end{array}$ & Seq. Line : & 3 \\
\hline Acq. Instrument & : LC & Location : & P2-C-63 \\
\hline njection Date & : 6/4/2019 1:09:36 PM & $\begin{array}{r}\text { Inj : } \\
\text { Inj Volume : }\end{array}$ & $\begin{array}{l}: \quad 1 \\
: 2.909 \mu \mathrm{l}\end{array}$ \\
\hline
\end{tabular}

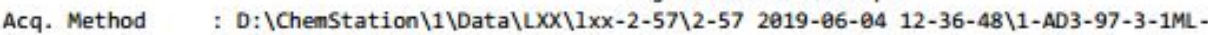
2UL-25C-15MIN. M

Last changed : 11/28/2018 10:54:45 AM by SYSTEM

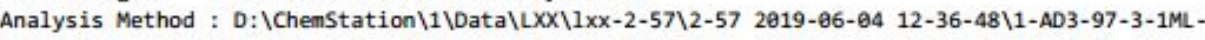

2UL-25C-15MIN.M (Sequence Method)

Last changed : 7/5/2019 1:52:28 PM by SYSTEM

(modified after loading)

Additional Info : Peak(s) manually integrated

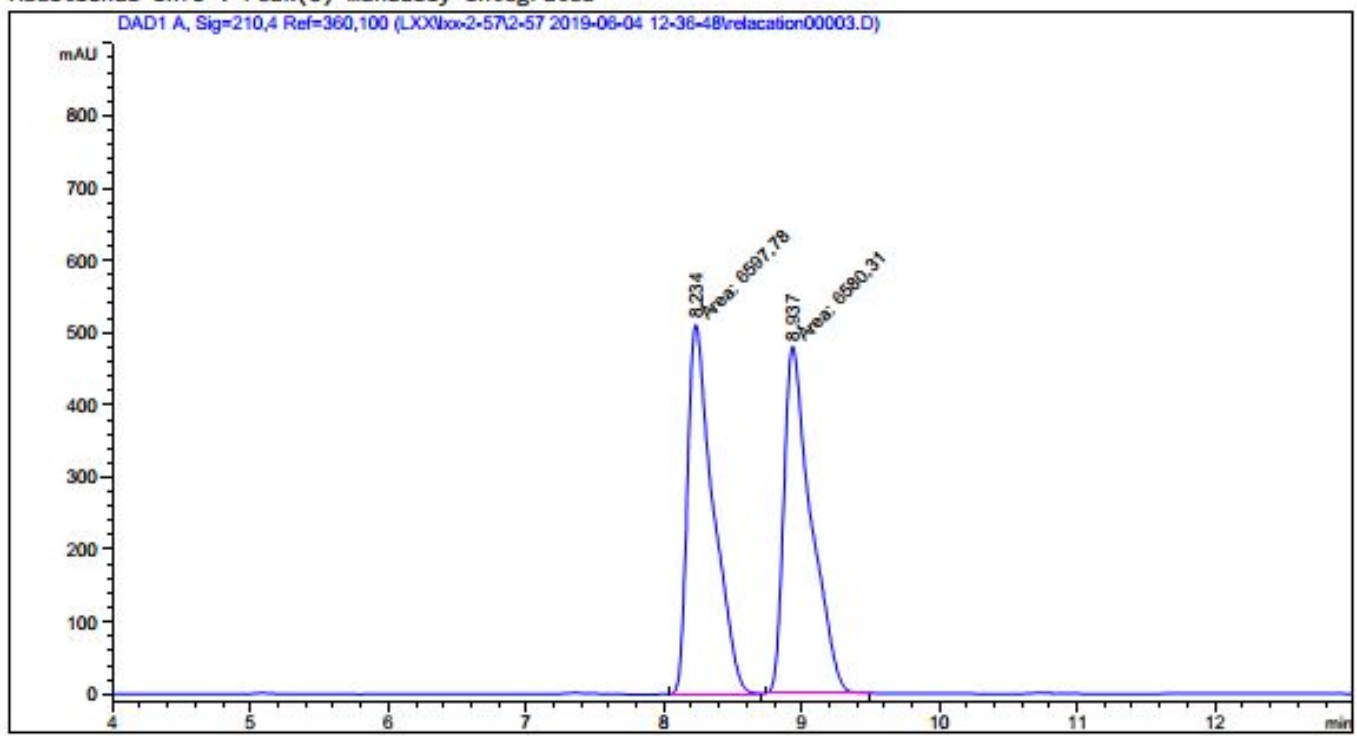

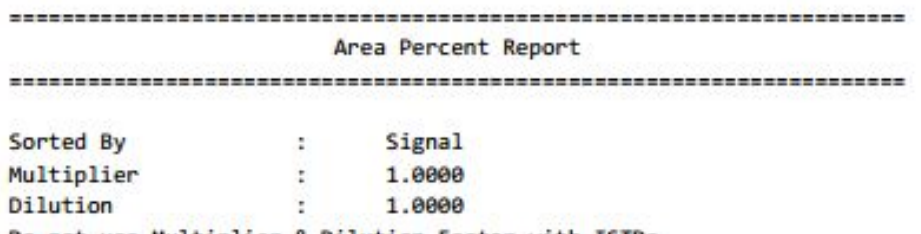

Do not use Multiplier \& Dilution Factor with ISTDs

Signal 1: DAD1 A, $S i g=210,4$ Ref $=360,100$

\begin{tabular}{cccccc}
$\begin{array}{c}\text { Peak RetTime Type } \\
\text { \# } \\
\text { [min] }\end{array}$ & $\begin{array}{c}\text { Width } \\
\text { [min] }\end{array}$ & $\begin{array}{c}\text { Area } \\
\text { [mAU*s] }\end{array}$ & $\begin{array}{c}\text { Height } \\
\text { [mAU] }\end{array}$ & $\begin{array}{c}\text { Area } \\
\%\end{array}$ \\
\hline 1 & $8.234 \mathrm{MM}$ & 0.2151 & 6597.78076 & 511.28738 & 50.0663 \\
2 & $8.937 \mathrm{MM}$ & 0.2282 & 6580.31445 & 480.61942 & 49.9337
\end{tabular}




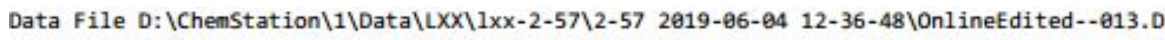
Sample Name: 57-3

\begin{tabular}{|c|c|c|c|}
\hline $\begin{array}{l}\text { Acq. Operator } \\
\text { Sample Operator }\end{array}$ & $\begin{array}{l}\text { : SYSTEM } \\
: \text { SYSTEM }\end{array}$ & Seq. Line & : 13 \\
\hline Acq. Instrument & : LC & Location & P2-B-93 \\
\hline Injection Date & : 6/4/2019 3:40:日2 PM & Inj & 1 \\
\hline
\end{tabular}

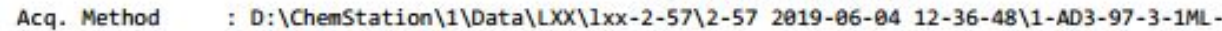
2UL-25C-15MIN. M

Last changed : 11/28/2018 10:54:45 AM by SYSTEM

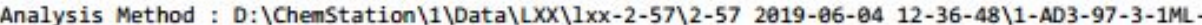
2UL-25C-15MIN.M (Sequence Method)

Last changed : 7/5/2019 1:50:12 PM by SYSTEM

(modified after loading)

Additional Info: Peak(s) manually integrated

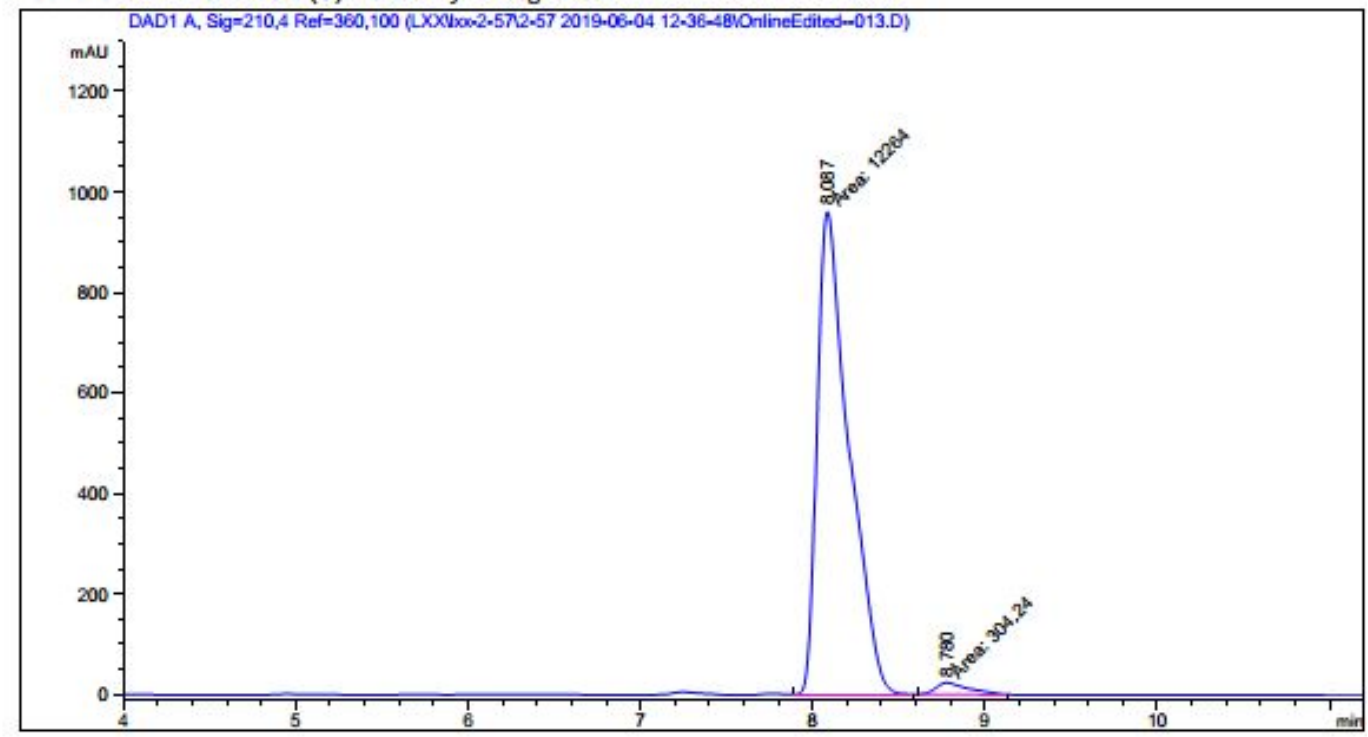

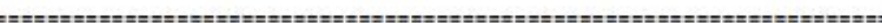

Area Percent Report

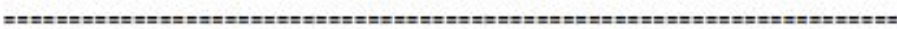

$\begin{array}{lll}\text { Sorted By } & : & \text { Signal } \\ \text { Multiplier } & : & 1.0000 \\ \text { Dilution } & : & 1.0000\end{array}$

Do not use Multiplier \& Dilution Factor with ISTDs

Signal 1: DAD1 A, Sig=210,4 Ref=360, 100

\begin{tabular}{|c|c|c|c|c|c|c|}
\hline $\begin{array}{c}\text { Peak } \\
\#\end{array}$ & $\begin{array}{l}\text { Ret Time } \\
\text { [min] }\end{array}$ & Type & $\begin{array}{l}\text { Width } \\
\text { [min] }\end{array}$ & $\begin{array}{c}\text { Area } \\
{\left[\mathrm{mAU}^{*} \mathrm{~s}\right]}\end{array}$ & $\begin{array}{l}\text { Height } \\
\text { [mAU] }\end{array}$ & $\begin{array}{c}\text { Area } \\
\%\end{array}$ \\
\hline$\ldots-1$ & 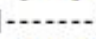 & & & |-...... & - & | \\
\hline 1 & 8.087 & $\mathrm{MM}$ & 0.2127 & $1.22648 \mathrm{e} 4$ & 961.09348 & 97.5793 \\
\hline 2 & 8.780 & $\mathrm{MM}$ & 0.2181 & 384.23962 & 23.24532 & 2.4207 \\
\hline Total & Is: & & & $1.25683 \mathrm{e} 4$ & 984.24880 & \\
\hline
\end{tabular}

LC 7/5/2019 1:50:36 PM SYSTEM 
<smiles>CC1(c2ccc(Br)cc2)C=CC(=O)CC1</smiles>

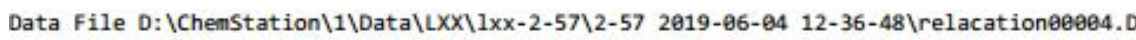
Sample Name: 57-4-RAC

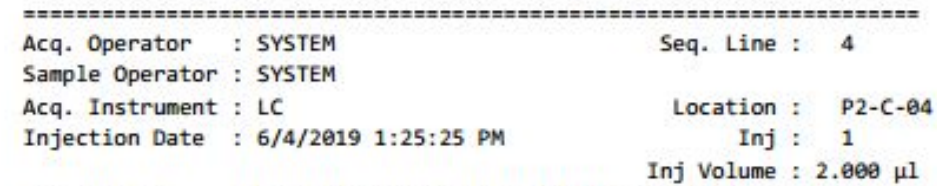

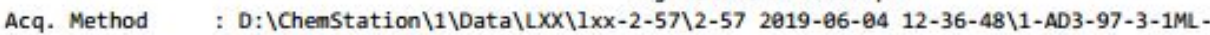
2UL-25C-15MIN. M

Last changed : 11/28/2018 10:54:45 AM by SYSTEM

Analysis Method: D:\ChemStation\1\Data\LXX\1XX-2-57\2-57 2019-66-64 12-36-48\1-AD3-97-3-1ML-

2UL-25C-15MIN.M (Sequence Method)

Last changed : 7/5/2019 2:31:44 PM by SYSTEM

(modified after loading)

Additional Info : Peak(s) manually integrated

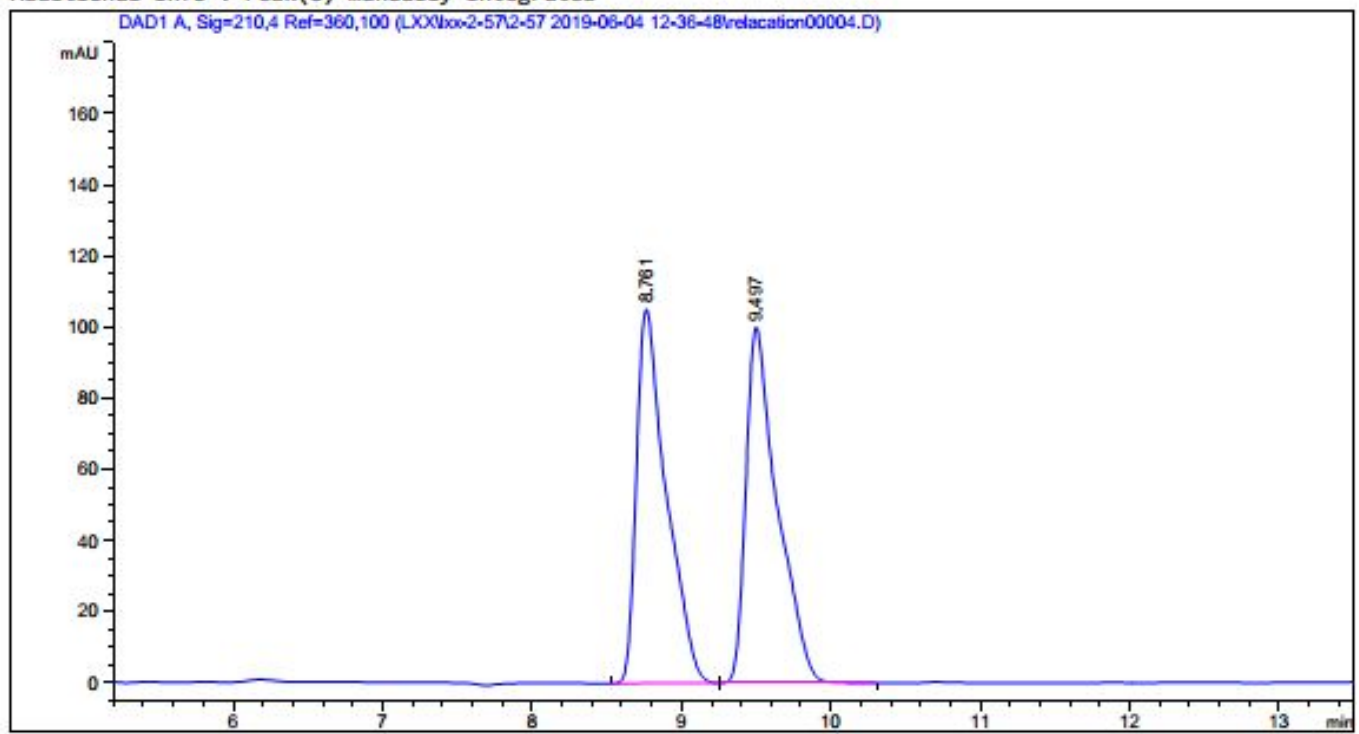

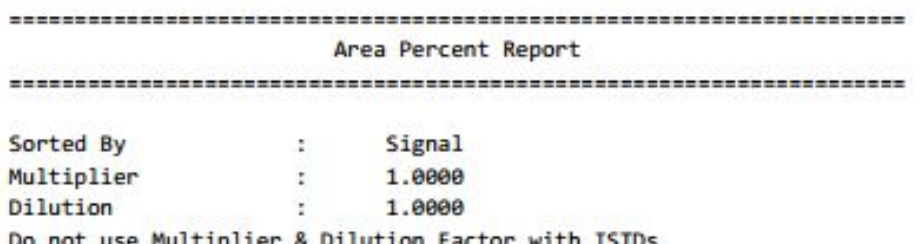

Do not use Multiplier \& Dilution Factor with ISTDs

Signal 1: DAD1 A, Sig $=210,4$ Ref $=360,100$

\begin{tabular}{|c|c|c|c|c|c|}
\hline $\begin{array}{c}\text { Peak } \\
\#\end{array}$ & $\begin{array}{l}\text { RetTime Type } \\
\text { [min] }\end{array}$ & $\begin{array}{l}\text { Width } \\
\text { [min] }\end{array}$ & $\begin{array}{c}\text { Area } \\
{\left[\mathrm{mAU}^{*} \mathrm{~s}\right]}\end{array}$ & $\begin{array}{l}\text { Height } \\
\text { [mAU] }\end{array}$ & $\begin{array}{c}\text { Area } \\
\text { \% }\end{array}$ \\
\hline$\ldots-1$ & $|-\ldots+|-\mid$ & . & |- 1-2. & . & ......... \\
\hline 1 & $8.761 \mathrm{BB}$ & 0.1970 & 1451.32251 & 105.16851 & 49.8586 \\
\hline 2 & 9.497 BB & 0.2984 & 1459.55383 & 99.95923 & 50.1414 \\
\hline Total & 1s: & & 2910.87634 & 205.12774 & \\
\hline
\end{tabular}




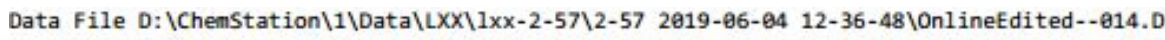
Sample Name: 57-4

\begin{tabular}{|c|c|c|c|}
\hline $\begin{array}{l}\text { Acq. Operator } \\
\text { Sample Operator }\end{array}$ & $\begin{array}{l}\text { : SYSTEM } \\
\text { : SYSTEM }\end{array}$ & Seq. Line & $: 14$ \\
\hline Acq. Instrument & : LC & Location & P2-B-64 \\
\hline Injection Date & : 6/4/2019 3:55:52 PM & $\begin{array}{r}\text { Inj } \\
\text { Inj Volume }\end{array}$ & $\begin{array}{l}: \quad 1 \\
: 2.000 \mu 1\end{array}$ \\
\hline
\end{tabular}

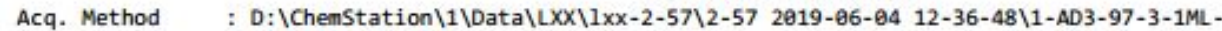
2UL $-25 \mathrm{C}-15 \mathrm{MIN} . \mathrm{M}$

Last changed : 6/4/2019 4:09:42 PM by SYSTEM (modified after loading)

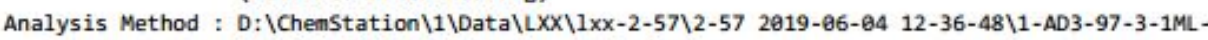
2UL-25C-15MIN.M (Sequence Method)

Last changed : 7/5/2019 2:29:41 PM by SYSTEM (modified after loading)

Additional Info: Peak(s) manually integrated

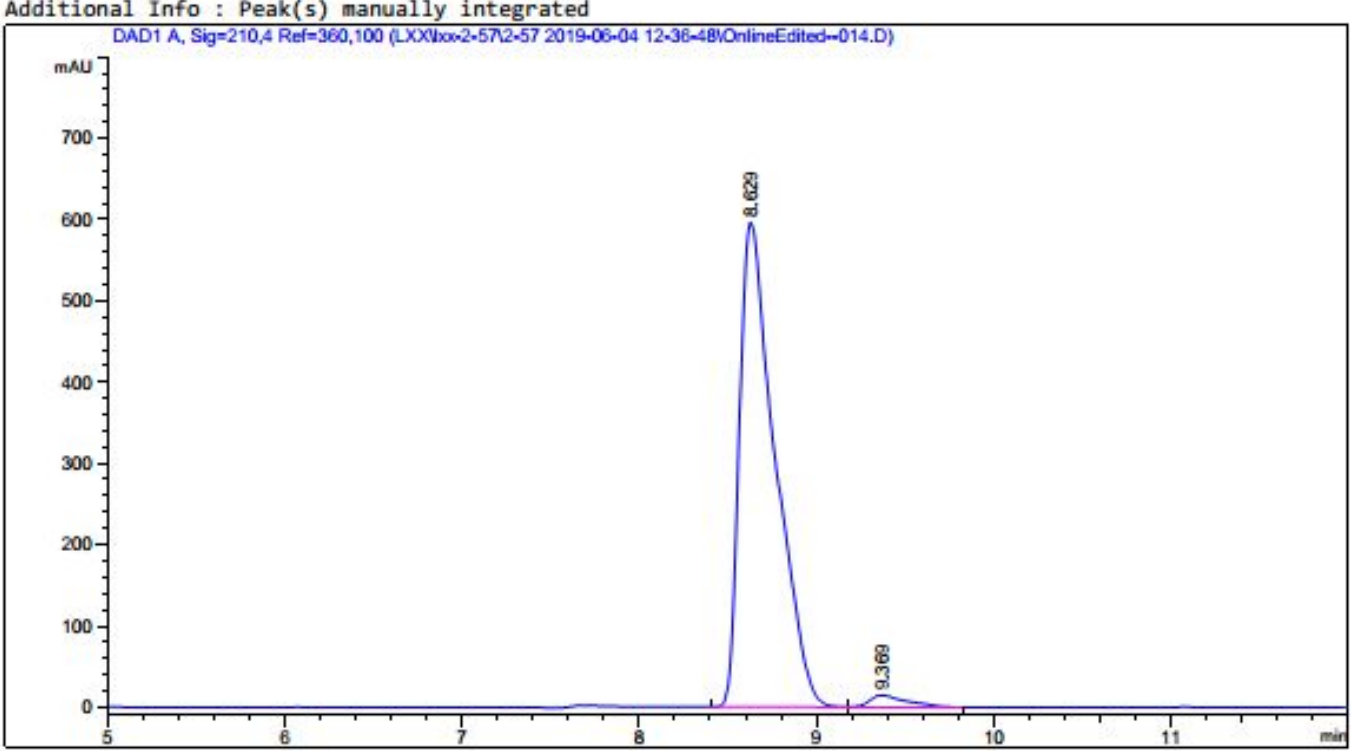

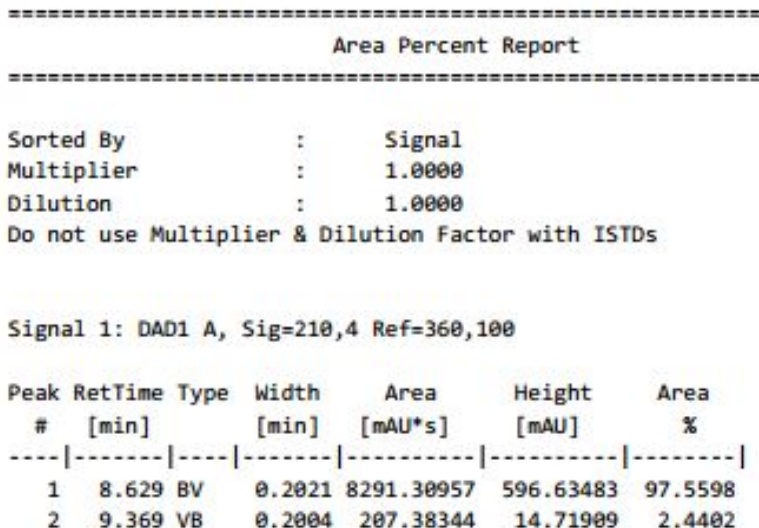


<smiles>COc1cccc([C@]2(C)C=CC(=O)CC2)c1</smiles>

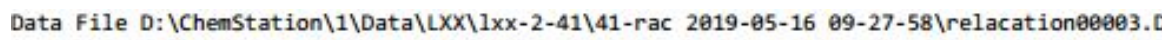
Sample Name: m-OCH3-Rac

\begin{tabular}{|c|c|c|c|}
\hline $\begin{array}{l}\text { Acq. Operator } \\
\text { Sample Operator }\end{array}$ & $\begin{array}{l}\text { : SYSTEM } \\
: \text { SYSTEM }\end{array}$ & Seq. Line : & 3 \\
\hline Acq. Instrument & $: L C$ & Location : & P2-C-62 \\
\hline Injection Date & : 5/16/2019 10:01:31 AM & $\begin{array}{r}\text { Inj : } \\
\text { Inj Volume : }\end{array}$ & $\begin{array}{l}: \quad 1 \\
: 2.000 \mu 1\end{array}$ \\
\hline
\end{tabular}

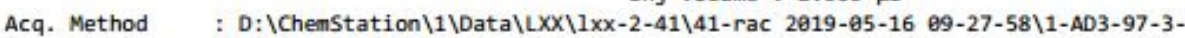
1 ML-2UL-25C-25MIN.M

Last changed : 11/28/2018 10:55:29 AM by SYSTEM

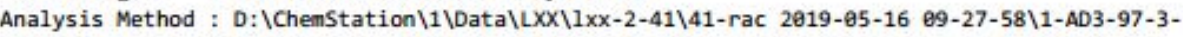

$1 \mathrm{ML}-2 \mathrm{UL}-25 \mathrm{C}-25 \mathrm{MIN} . \mathrm{M}$ (Sequence Method)

Last changed : 7/5/2019 2:20:46 PM by SYSTEM

(modified after loading)

Additional Info: Peak(s) manually integrated
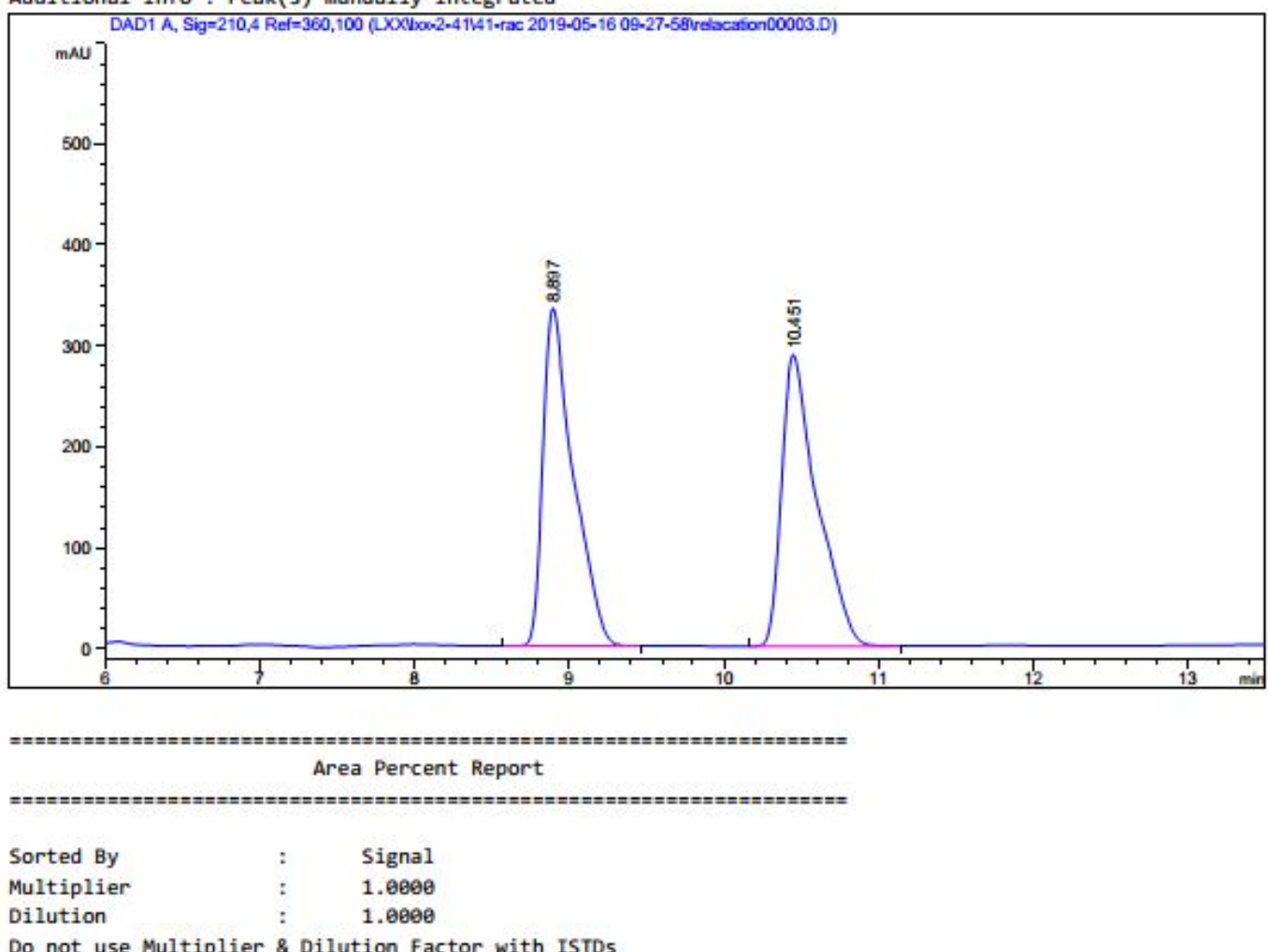

Do not use Multiplier \& Dilution Factor with ISTDs

Signal 1: DAD1 A, Sig $=210,4$ Ref $=360,100$

\begin{tabular}{|c|c|c|c|c|c|}
\hline $\begin{array}{c}\text { Peak } \\
\#\end{array}$ & $\begin{array}{l}\text { RetTime Type } \\
\text { [min] }\end{array}$ & $\begin{array}{l}\text { Width } \\
\text { [min] }\end{array}$ & $\begin{array}{c}\text { Area } \\
{\left[\mathrm{mAU}^{*} \mathrm{~s}\right]}\end{array}$ & $\begin{array}{l}\text { Height } \\
\text { [maU] }\end{array}$ & $\begin{array}{c}\text { Area } \\
\text { \% }\end{array}$ \\
\hline$\cdots-1$ & $\cdots$ & 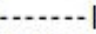 & |-....en & . & -1 \\
\hline 1 & $8.897 \mathrm{BB}$ & 0.1949 & 4560.27051 & 334.91098 & 49.8997 \\
\hline 2 & $10.451 \mathrm{BB}$ & 0.2269 & 4578.60645 & 288.97083 & 50.1003 \\
\hline Total & 1s: & & 9138.87695 & 623.88181 & \\
\hline
\end{tabular}




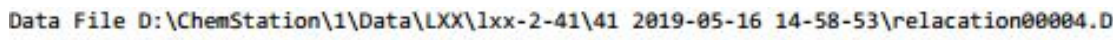
Sample Name: $41-3$

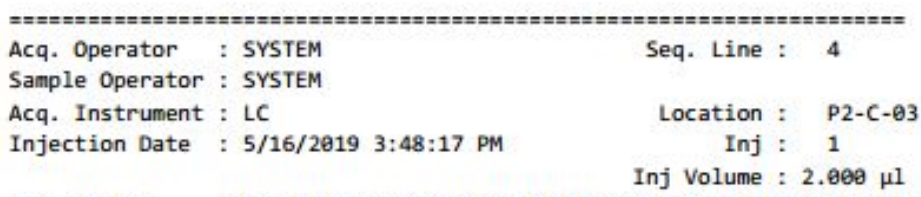

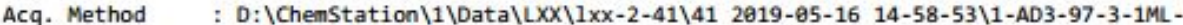
2UL-25C-25MIN. M

Last changed : 5/16/2019 4:02:55 PM by SYSTEM (modified after loading)

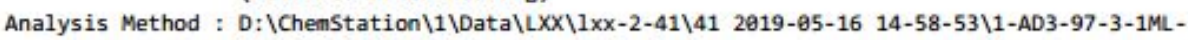
2UL-25C-25MIN. M (Sequence Method)

Last changed : 7/5/2019 2:19:84 PM by SYSTEM (modified after loading)

Additional Info: Peak(s) manually integrated

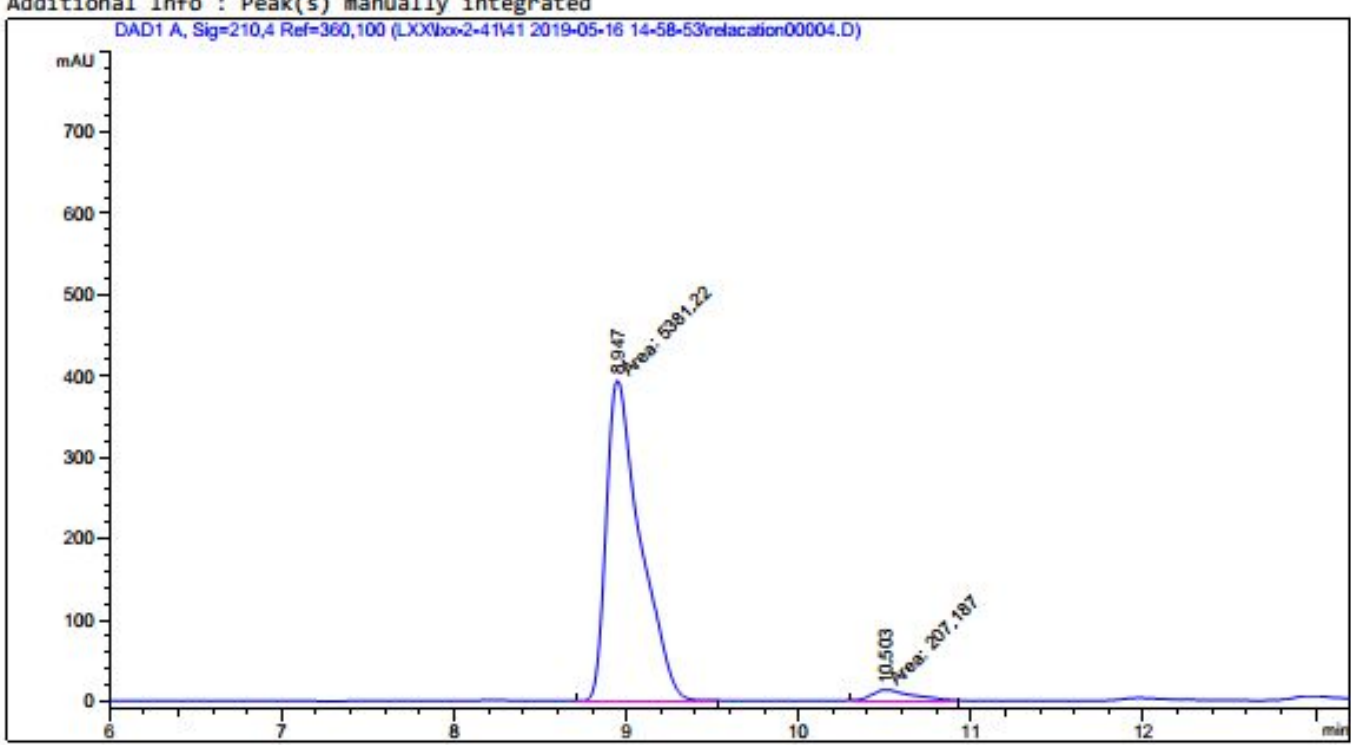

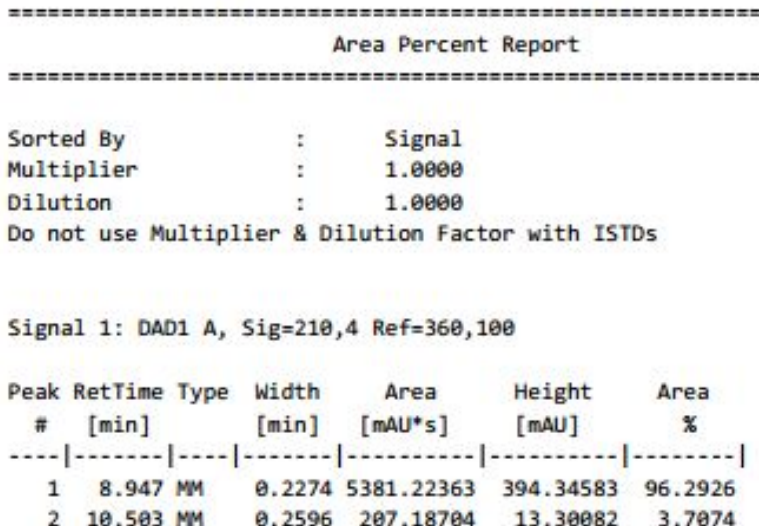


<smiles>COc1ccccc1C1(C)C=CC(=O)CC1</smiles>

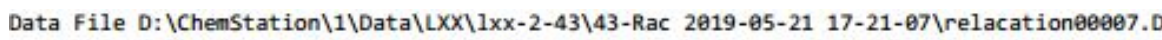
Sample Name: o-CH3-Rac

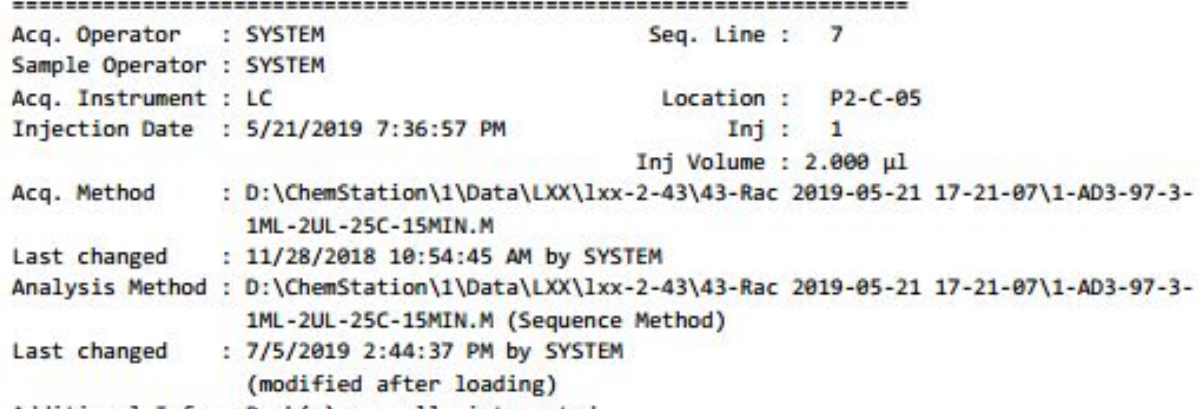

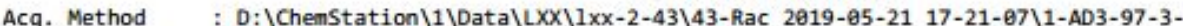
1ML-2UL-25C-15MIN. M

Last changed : 11/28/2018 10:54:45 AM by SYSTEM

Analysis Method : D: \ChemStation\1\Data\LXX\1XX-2-43\43-Rac 2019-95-21 17-21-97\1-AD3-97-31ML-2UL-25C-15MIN.M (Sequence Method)

Last changed : 7/5/2019 2:44:37 PM by SYSTEM

(modified after loading)

Additional Info: Peak(s) manually integrated
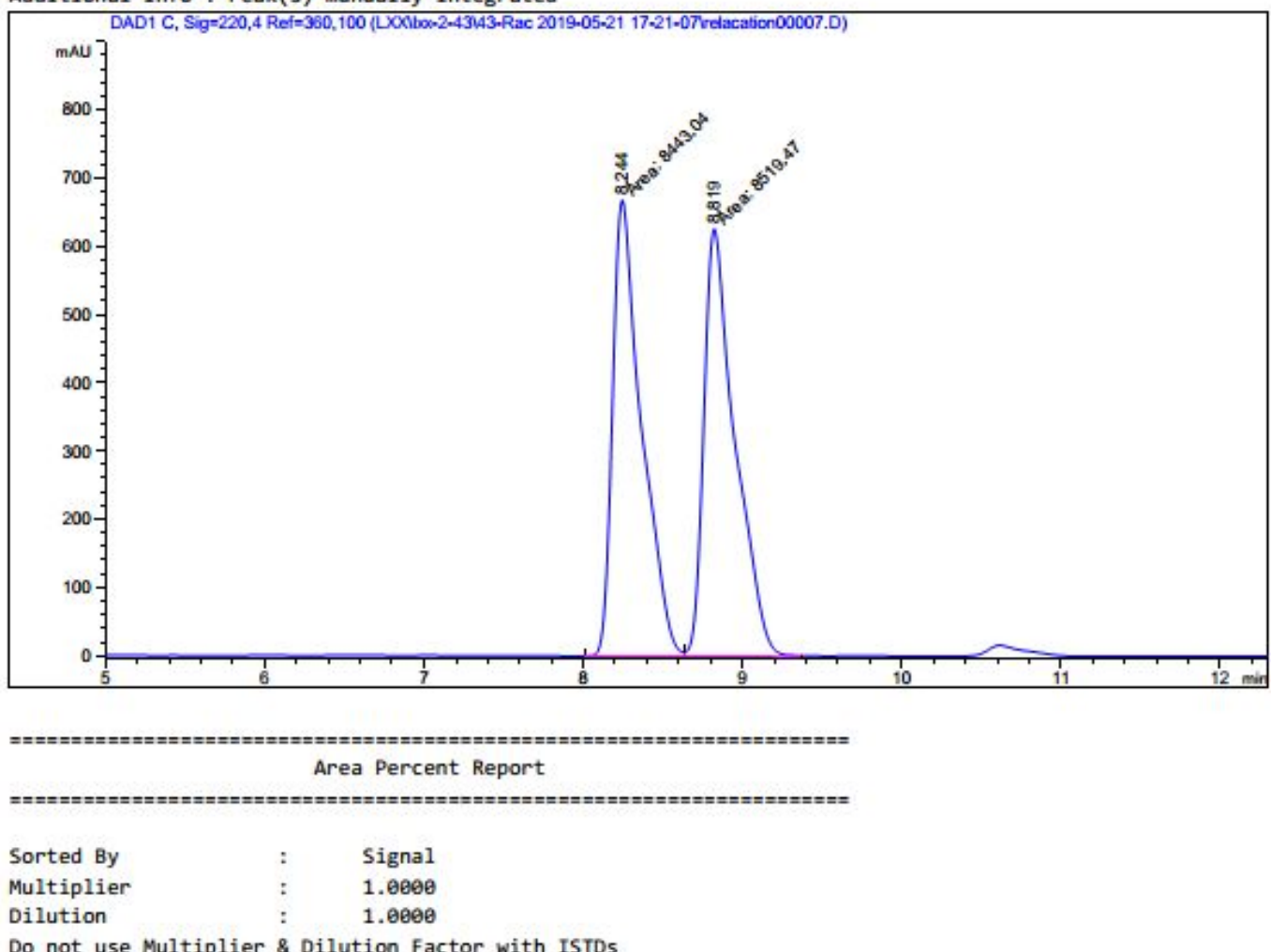

Do not use Multiplier \& Dilution Factor with ISTDs

Signal 1: DAD1 C, Sig $=220,4$ Ref $=360,100$

\begin{tabular}{|c|c|c|c|c|c|c|}
\hline $\begin{array}{c}\text { Peak } \\
\#\end{array}$ & $\begin{array}{c}\text { RetTime } \\
\text { [min] }\end{array}$ & Type & $\begin{array}{l}\text { Width } \\
\text { [min] }\end{array}$ & $\begin{array}{c}\text { Area } \\
{\left[\mathrm{mAU}^{*} \mathrm{~s}\right]}\end{array}$ & $\begin{array}{l}\text { Height } \\
\text { [mAU] }\end{array}$ & $\begin{array}{c}\text { Area } \\
\text { \% }\end{array}$ \\
\hline$\ldots-1$ & | & & . & |-......... & $|-\ldots+2.0|$. & - \\
\hline 1 & 8.244 & MF & 0.2105 & 8443.04004 & 668.54712 & 49.7747 \\
\hline 2 & 8.819 & FM & 0.2266 & 8519.46777 & 626.62897 & 50.2253 \\
\hline Total & 1s: & & & $1.69625 \mathrm{e} 4$ & 1295.17609 & \\
\hline
\end{tabular}




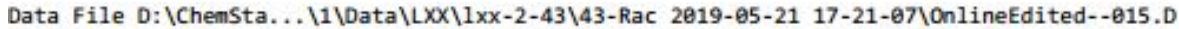
Sample Name: $43-10$

\begin{tabular}{|c|c|c|c|}
\hline $\begin{array}{l}\text { Acq. Operator } \\
\text { Sample Operator }\end{array}$ & $\begin{array}{l}\text { : SYSTEM } \\
: \text { SYSTEM }\end{array}$ & Seq. Line : & : 15 \\
\hline Acq. Instrument & : LC & Location : & P2-D-10 \\
\hline Injection Date & : 5/21/2019 9:53:46 PM & $\begin{array}{r}\text { Inj : } \\
\text { Inj Volume : }\end{array}$ & $\begin{array}{l}: \quad 1 \\
: 2.000 \mu 1\end{array}$ \\
\hline
\end{tabular}

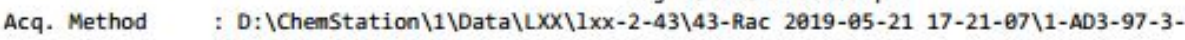
1ML-2UL-25C-15MIN.M

Last changed : 11/28/2018 10:54:45 AM by SYSTEM

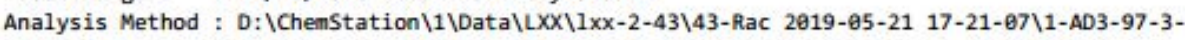

1ML-2UL-25C-15MIN.M (Sequence Method)

Last changed : 7/5/2019 2:42:53 PM by SYSTEM

(modified after loading)

Additional Info: Peak(s) manually integrated

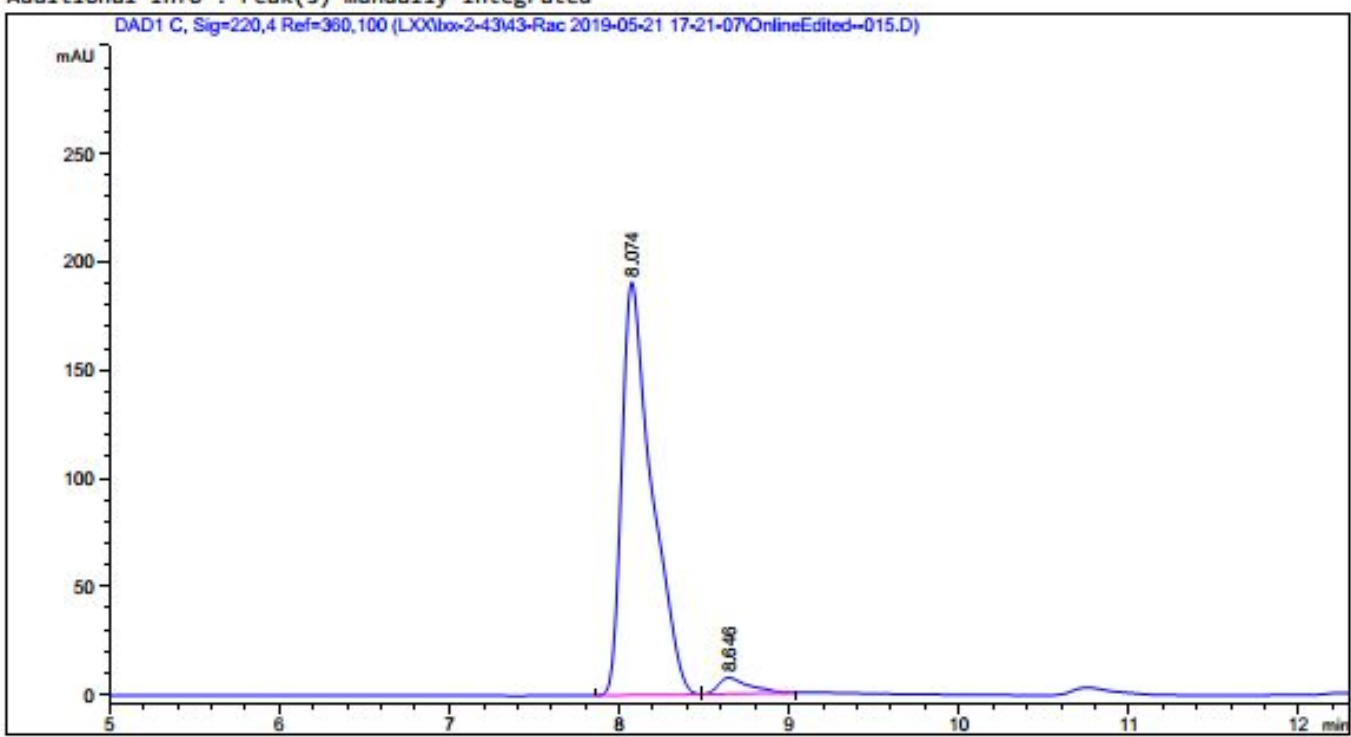

Area Percent Report

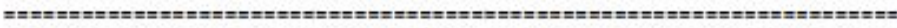

Sorted By : : Signal

Multiplier : 1.0000

Dilution : 1.0000

Do not use Multiplier \& Dilution Factor with ISTDs

Signal 1: DAD1 C, Sig $=220,4$ Ref $=360,100$

\begin{tabular}{|c|c|c|c|c|c|}
\hline $\begin{array}{c}\text { Peak } \\
\#\end{array}$ & $\begin{array}{l}\text { RetTime Type } \\
\text { [min] }\end{array}$ & $\begin{array}{l}\text { Width } \\
\text { [min] }\end{array}$ & $\begin{array}{c}\text { Area } \\
{\left[\mathrm{mAU}^{*} \mathrm{~s}\right]}\end{array}$ & $\begin{array}{l}\text { Height } \\
\text { [maU] }\end{array}$ & $\begin{array}{c}\text { Area } \\
\text { \% }\end{array}$ \\
\hline 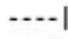 & 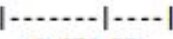 & (n....... & | & | & - \\
\hline 1 & 8.074 BB & 0.1747 & 2333.33301 & 190.74590 & 96.1525 \\
\hline 2 & $8.646 \mathrm{BB}$ & 0.1812 & 93.36819 & 7.40125 & 3.8475 \\
\hline Tot & : & & $2426.7012 \theta$ & 198.14715 & \\
\hline
\end{tabular}


2j

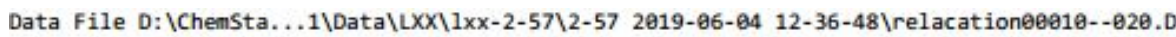
Sample Name: 57-9-RAC

\begin{tabular}{|c|c|c|c|}
\hline $\begin{array}{l}\text { Acq. Operator } \\
\text { Sample Operator }\end{array}$ & $\begin{array}{l}\text { : SYSTEM } \\
: \text { SYSTEM }\end{array}$ & Seq. Line & $: 20$ \\
\hline Acq. Instrument & : LC & Location & P2-C-69 \\
\hline njection Date & : 6/4/2019 5:28:27 PM & $\begin{array}{r}\text { Inj } \\
\text { Inj Volume }\end{array}$ & $\begin{array}{l}: \quad 1 \\
: 2.909 \mu 1\end{array}$ \\
\hline
\end{tabular}

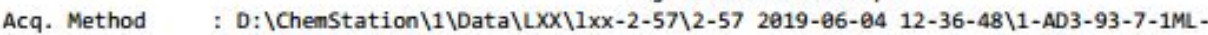
2UL-25C-49MIN. M

Last changed : 1/22/2019 9:44:48 AM by SYSTEM

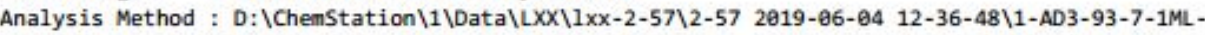

2UL-25C-46MIN.M (Sequence Method)

Last changed : 7/5/2019 2:56:06 PM by SYSTEM

(modified after loading)

Additional Info: Peak(s) manually integrated

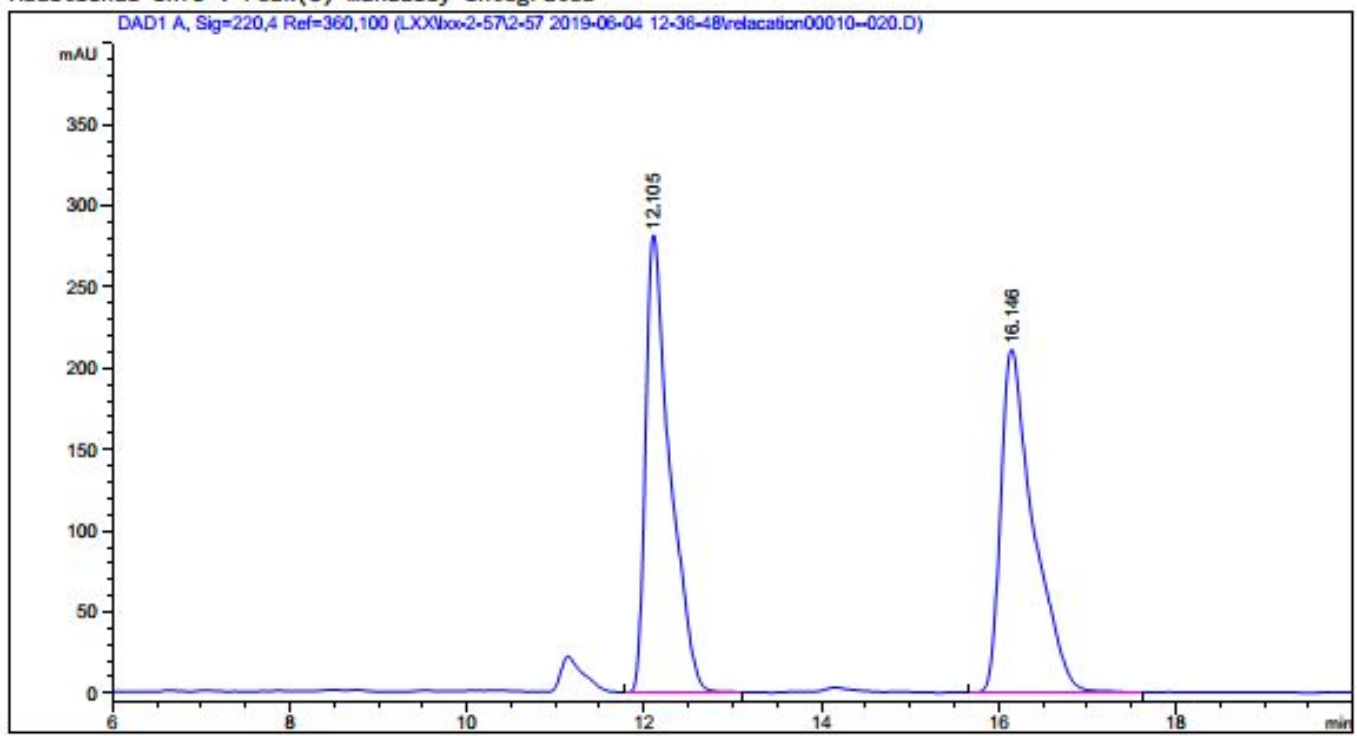

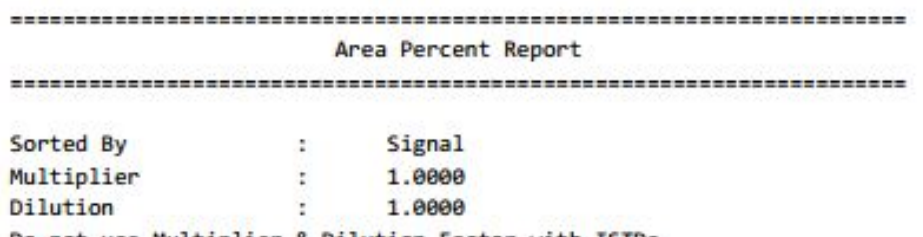

Do not use Multiplier \& Dilution Factor with ISTDs

Signal 1: DAD1 A, $S i g=220,4$ Ref $=360,100$

\begin{tabular}{|c|c|c|c|c|c|}
\hline $\begin{array}{c}\text { Peak } \\
\#\end{array}$ & $\begin{array}{l}\text { RetTime Type } \\
\text { [min] }\end{array}$ & $\begin{array}{l}\text { Width } \\
\text { [min] }\end{array}$ & $\begin{array}{c}\text { Area } \\
{\left[\mathrm{mAU}^{*} \mathrm{~s}\right]}\end{array}$ & $\begin{array}{l}\text { Height } \\
\text { [maU] }\end{array}$ & $\begin{array}{c}\text { Area } \\
\text { \% }\end{array}$ \\
\hline$\cdots-1$ & $|\cdots|$ & & n. & $\cdots$ & . \\
\hline 1 & $12.105 \mathrm{BB}$ & 0.2744 & 5360.83154 & 281.17859 & 49.7452 \\
\hline 2 & $16.146 \mathrm{BB}$ & 0.3673 & 5415.74951 & 211.08298 & 50.2548 \\
\hline \multicolumn{3}{|c|}{ Totals : } & $1.07766 \mathrm{e} 4$ & 492.26157 & \\
\hline
\end{tabular}

LC 7/5/2019 2:56:11 PM SYSTEM 


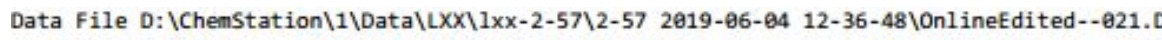
Sample Name: 57-9

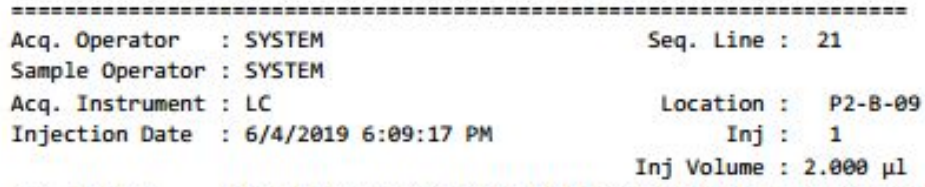

Acq. Method : D: \ChemStation\1\Data\LXX\1XX-2-57\2-57 2019-96-04 12-36-48\1-AD3-93-7-1ML2UL-25C-46MIN. M

Last changed : 6/4/2019 6:45:28 PM by SYSTEM (modified after loading)

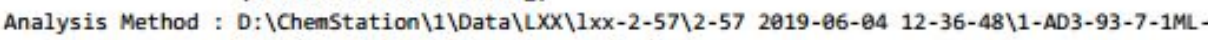
2UL-25C-40MIN.M (Sequence Method)

Last changed : 7/23/2019 8:06:44 PM by SYSTEM (modified after loading)

Additional Info: Peak(s) manually integrated

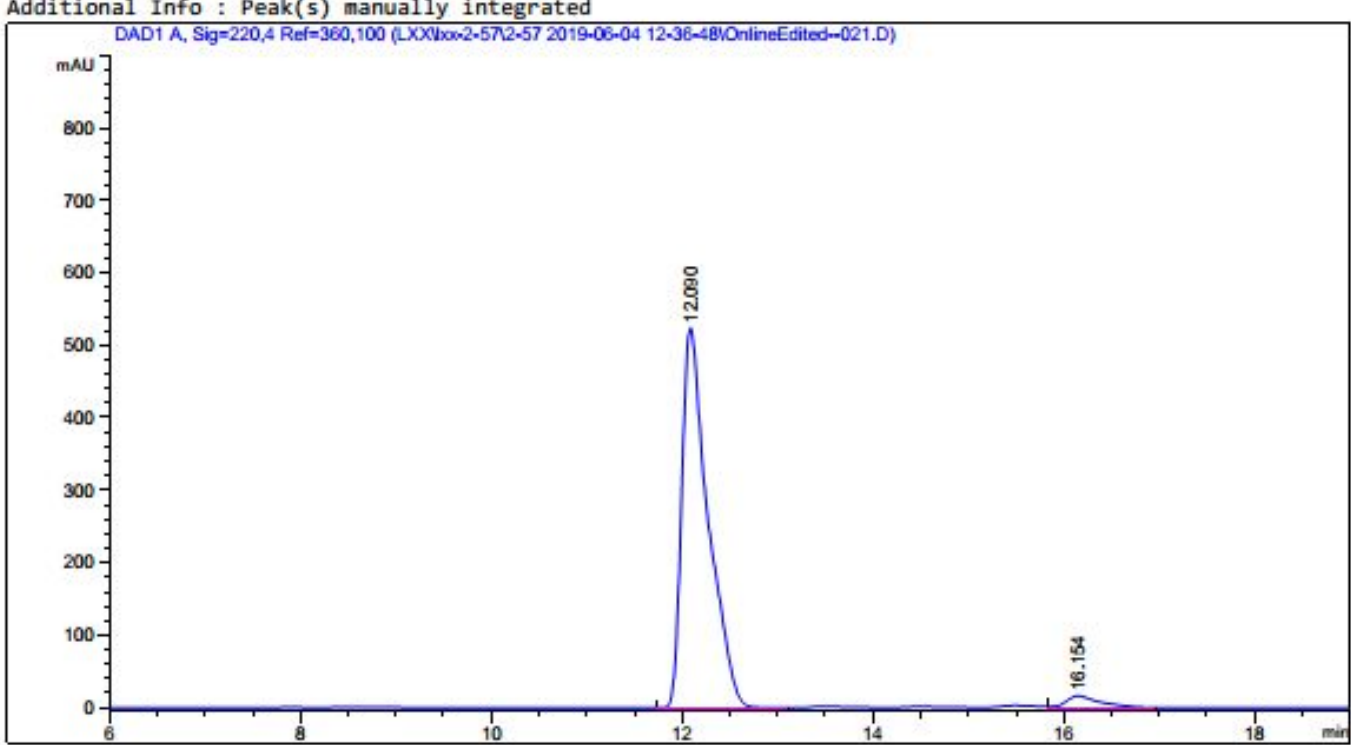

\begin{tabular}{|c|c|c|}
\hline Sorted By & : & Sign \\
\hline Multiplier & : & $1 . \theta 6$ \\
\hline Dilution & : & 1.6 \\
\hline
\end{tabular}

Do not use Multiplier \& Dilution Factor with ISTDs

Signal 1: DAD1 A, Sig $=220,4$ Ref $=360,100$

\begin{tabular}{|c|c|c|c|c|c|}
\hline $\begin{array}{c}\text { Peak } \\
\#\end{array}$ & $\begin{array}{l}\text { RetTime Type } \\
\text { [min] }\end{array}$ & $\begin{array}{l}\text { Width } \\
\text { [min] }\end{array}$ & $\begin{array}{c}\text { Area } \\
\text { [mAU*s] }\end{array}$ & $\begin{array}{l}\text { Height } \\
\text { [mAU] }\end{array}$ & $\begin{array}{c}\text { Area } \\
\text { \% }\end{array}$ \\
\hline 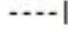 & & & & & \\
\hline 1 & $12.090 \mathrm{BB}$ & 0.2779 & $1.00602 \mathrm{e} 4$ & 524.23938 & 96.0616 \\
\hline 2 & $16.154 \mathrm{VB}$ & 0.3635 & 412.45706 & 16.17408 & 3.9384 \\
\hline
\end{tabular}


<smiles>CC1(c2ccc3ccccc3c2)C=CC(=O)CC1</smiles>

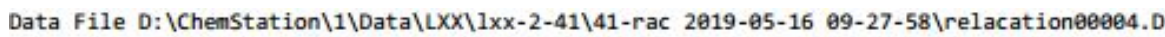
Sample Name: nai-Rac

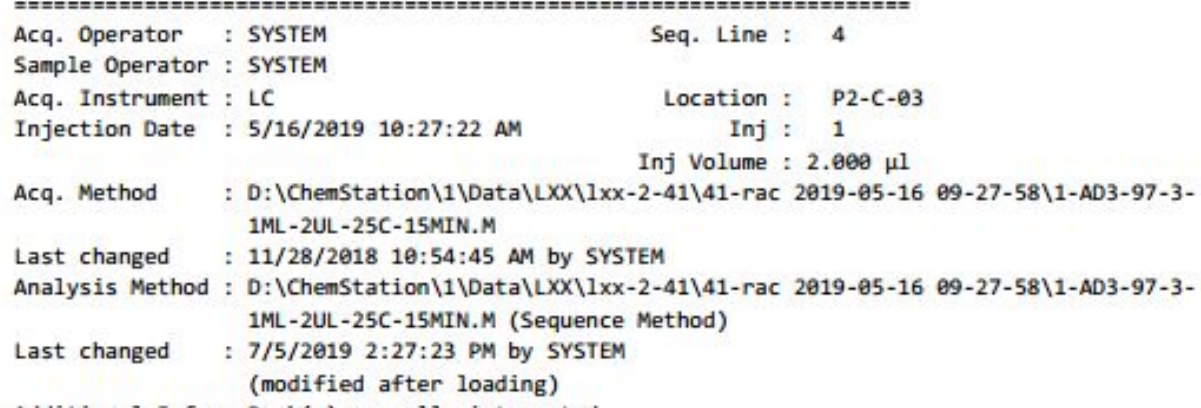

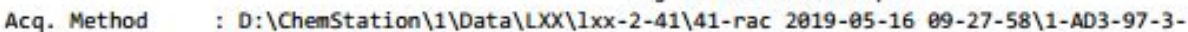
1ML-2UL-25C-15MIN. M

Last changed : 11/28/2018 10:54:45 AM by SYSTEM

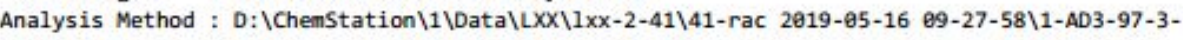
1ML-2UL-25C-15MIN.M (Sequence Method)

Last changed : 7/5/2019 2:27:23 PM by SYSTEM (modified after loading)

Additional Info : Peak(s) manually integrated

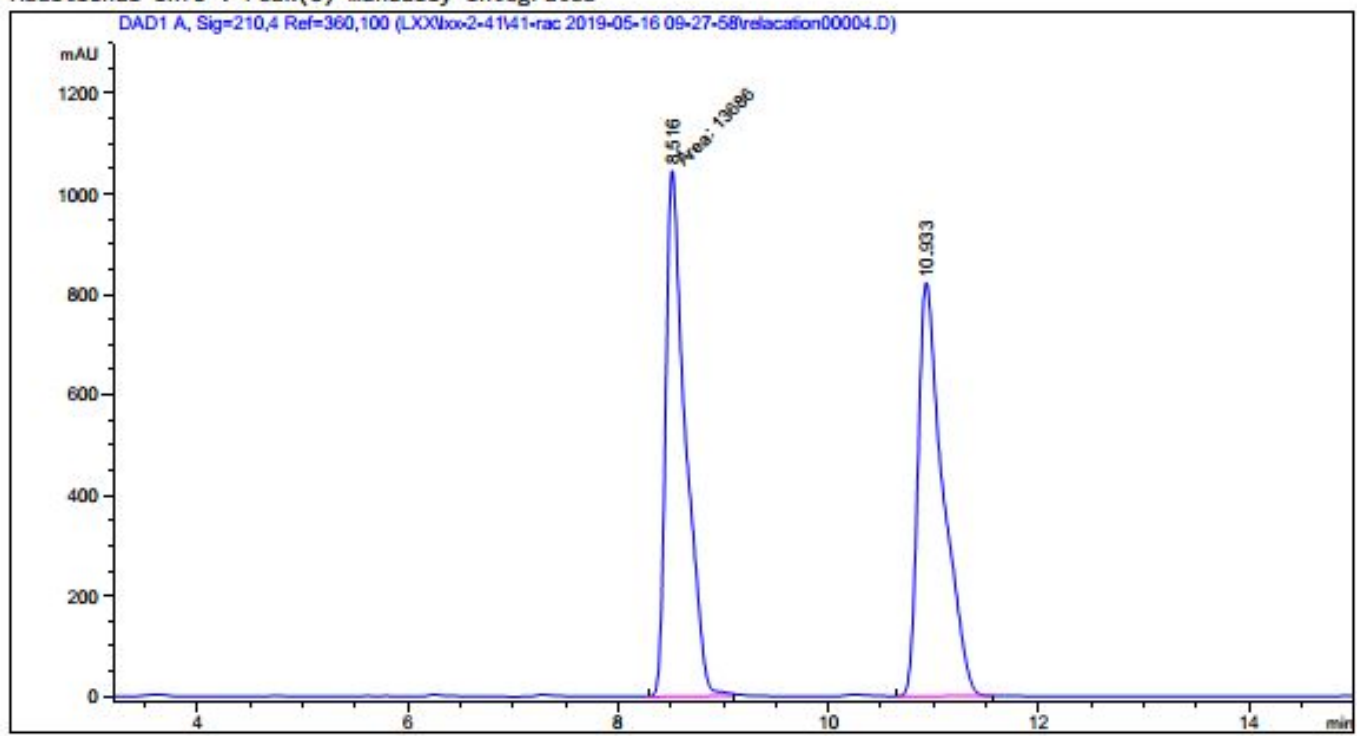

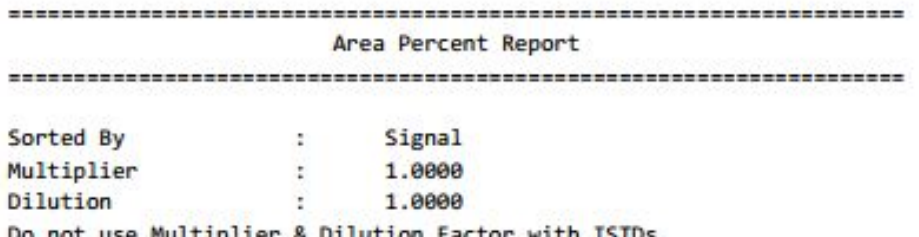

Do not use Multiplier \& Dilution Factor with ISTDs

Signal 1: DAD1 A, $S i g=210,4$ Ref $=360,100$

\begin{tabular}{|c|c|c|c|c|c|c|}
\hline $\begin{array}{c}\text { Peak } \\
\#\end{array}$ & $\begin{array}{l}\text { RetTime } \\
\text { [min] }\end{array}$ & Type & $\begin{array}{l}\text { Width } \\
\text { [min] }\end{array}$ & $\begin{array}{c}\text { Area } \\
{\left[\mathrm{mAU}^{*} \mathrm{~s}\right]}\end{array}$ & $\begin{array}{l}\text { Height } \\
\text { [mAU] }\end{array}$ & $\begin{array}{c}\text { Area } \\
\%\end{array}$ \\
\hline$\cdots$ & |....... & 1- & $\ldots-\ldots$ & | & |1-n....... & -.......... \\
\hline 1 & 8.516 & MM & 0.2177 & $1.3686 \mathrm{be} 4$ & 1047.62280 & 50.1079 \\
\hline 2 & 10.933 & BB & 0.2371 & $1.36271 \mathrm{e} 4$ & 823.17413 & 49.8921 \\
\hline Tota & 1s: & & & $2.73132 \mathrm{e} 4$ & 1870.79694 & \\
\hline
\end{tabular}




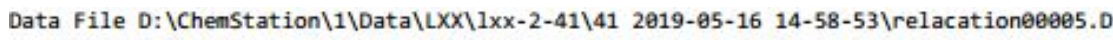
Sample Name: $41-4$

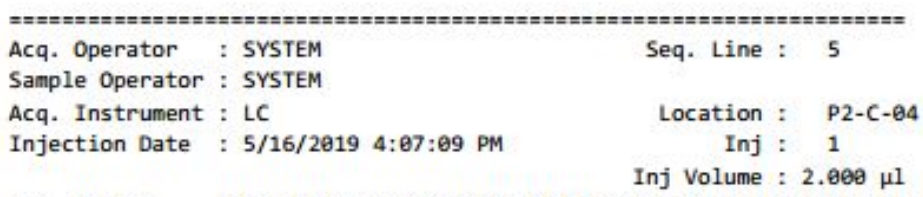

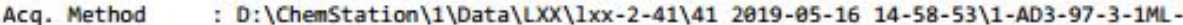
2UL -25C-15MIN.M

Last changed : 11/28/2018 10:54:45 AM by SYSTEM

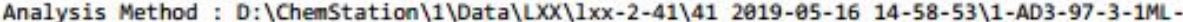
2UL-25C-15MIN.M (Sequence Method)

Last changed : 7/5/2019 2:24:84 PM by SYSTEM (modified after loading)

Additional Info: Peak(s) manually integrated

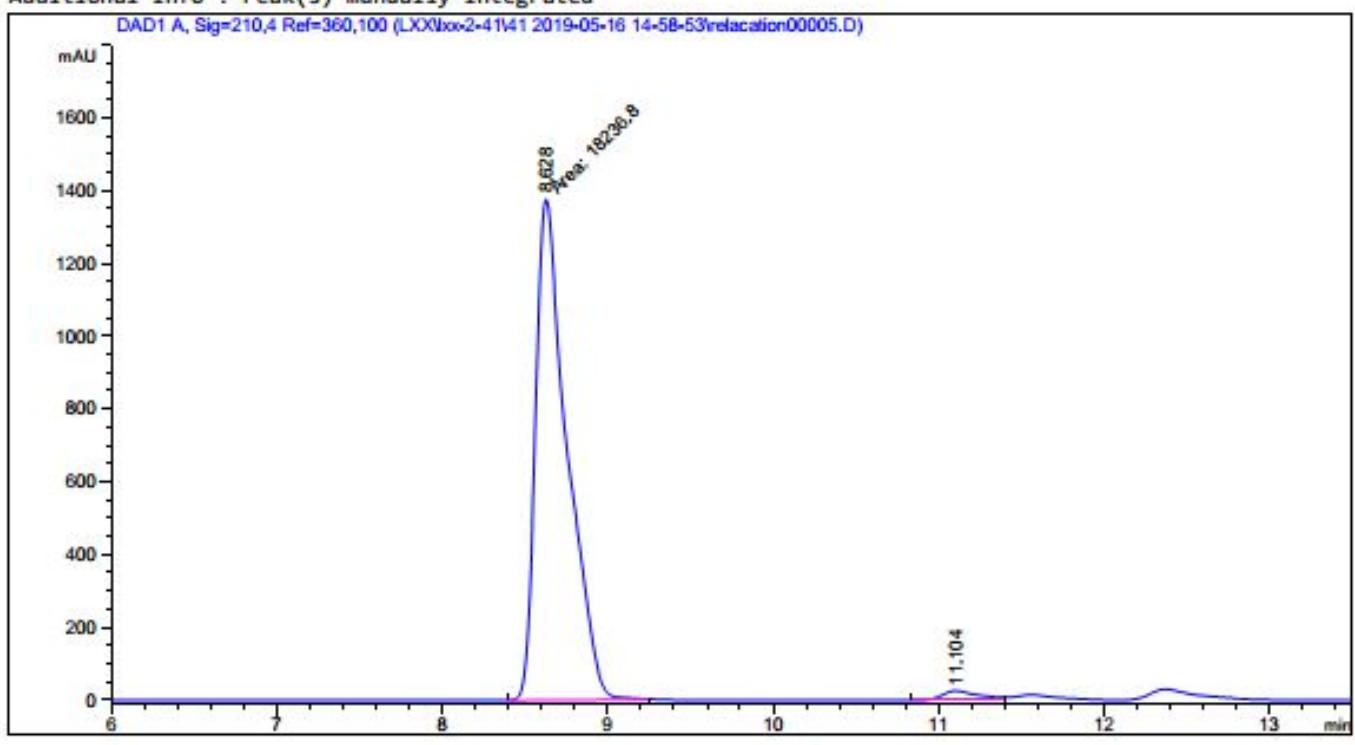

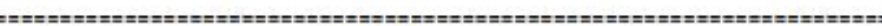

Area Percent Report

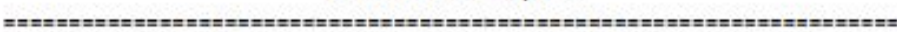

$\begin{array}{lll}\text { Sorted By } & : & \text { Signal } \\ \text { Multiplier } & : & 1.0000 \\ \text { Dilution } & : & 1.0000\end{array}$

Do not use Multiplier \& Dilution Factor with ISTDs

Signal 1: DAD1 A, Sig=210,4 Ref $=360,100$

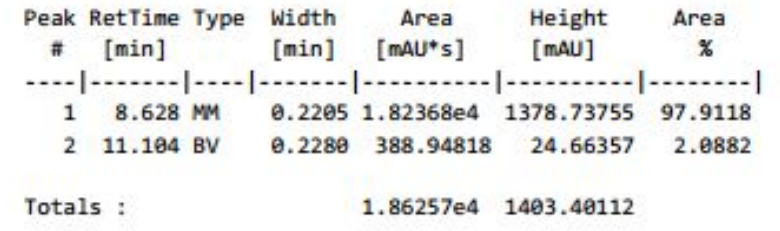


<smiles>CC1(C2CCCCC2)C=CC(=O)CC1</smiles>

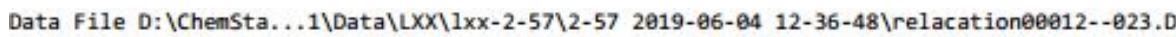
Sample Name: 57-10-RAC

\begin{tabular}{|c|c|c|c|}
\hline $\begin{array}{l}\text { Acq. Operator } \\
\text { Sample Operator }\end{array}$ & $\begin{array}{l}: \text { SYSTEM } \\
: \text { SYSTEM }\end{array}$ & Seq. Line & $: 23$ \\
\hline Acq. Instrument & : LC & Location & P2-C-10 \\
\hline njection Date & : 6/4/2019 7:02:32 PM & $\begin{array}{r}\text { Inj } \\
\text { Inj Volume }\end{array}$ & $\begin{array}{l}: \quad 1 \\
: 2.909 \mu \mathrm{l}\end{array}$ \\
\hline
\end{tabular}

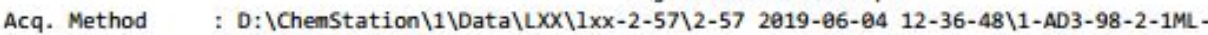
2UL-25C-15MIN. M

Last changed : 5/21/2019 5:17:05 PM by SYSTEM

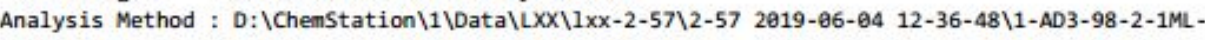

2UL-25C-15MIN.M (Sequence Method)

Last changed : 7/5/2019 3:02:54 PM by SYSTEM

(modified after loading)

Additional Info: Peak(s) manually integrated
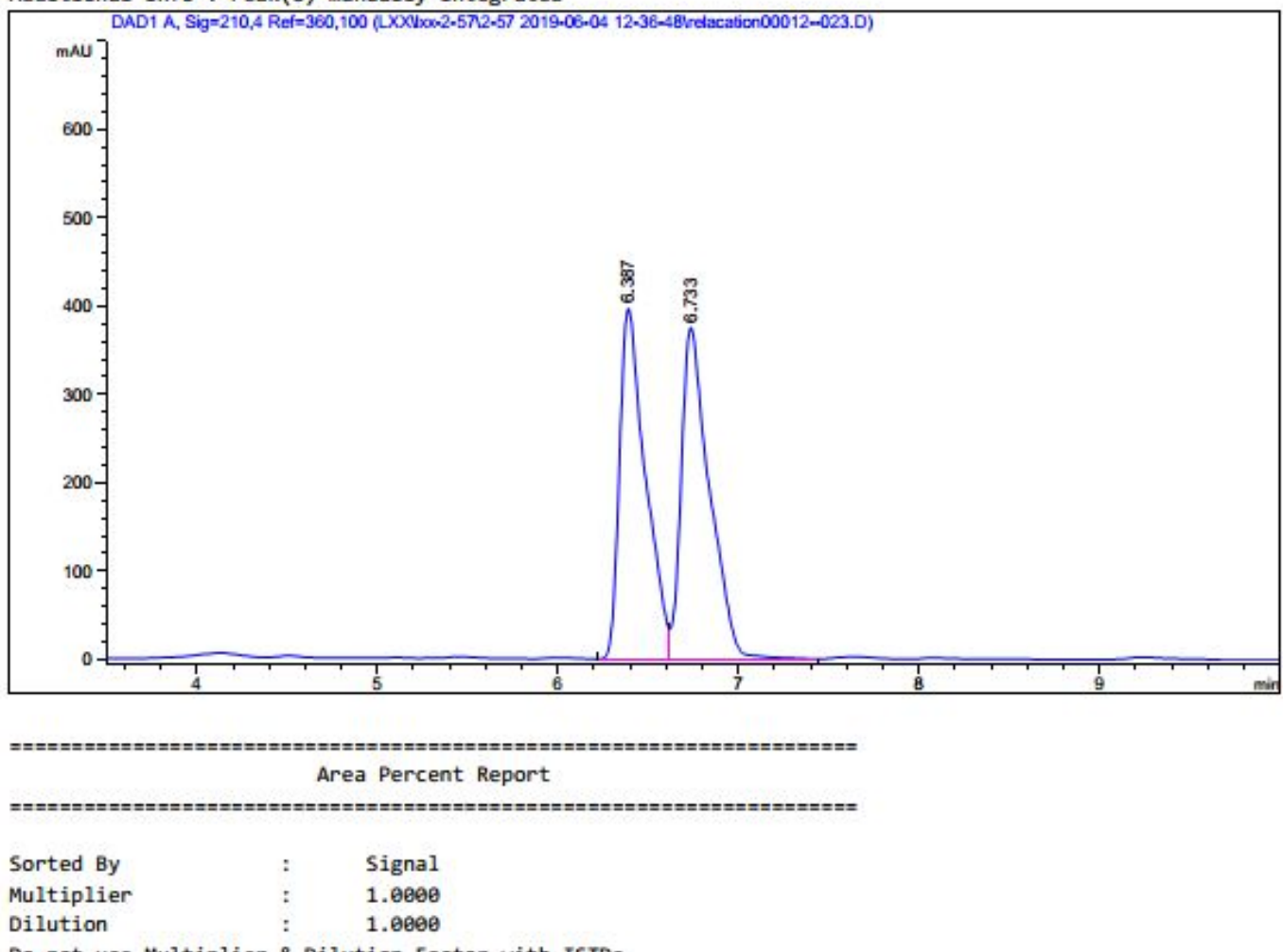

Do not use Multiplier \& Dilution Factor with ISTDs

Signal 1: DAD1 A, Sig $=210,4$ Ref $=360,100$

\begin{tabular}{|c|c|c|c|c|c|}
\hline $\begin{array}{c}\text { Peak } \\
\#\end{array}$ & $\begin{array}{l}\text { RetTime Type } \\
\text { [min] }\end{array}$ & $\begin{array}{l}\text { Width } \\
\text { [min] }\end{array}$ & $\begin{array}{c}\text { Area } \\
{\left[\mathrm{mAU}^{*} \mathrm{~s}\right]}\end{array}$ & $\begin{array}{l}\text { Height } \\
\text { [maU] }\end{array}$ & $\begin{array}{c}\text { Area } \\
\text { \% }\end{array}$ \\
\hline$\cdots-1$ & {$[-5$} & - & | & . & - \\
\hline 1 & $6.387 \mathrm{BV}$ & 0.1441 & 3952.09351 & 397.13107 & 49.2690 \\
\hline 2 & $6.733 \mathrm{VB}$ & 0.1545 & 4069.37012 & 375.39035 & 50.7310 \\
\hline Total & 1s: & & 8021.46362 & 772.52142 & \\
\hline
\end{tabular}




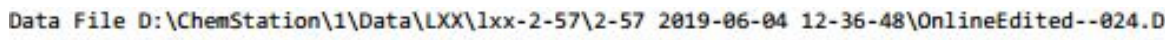
Sample Name: 57-10

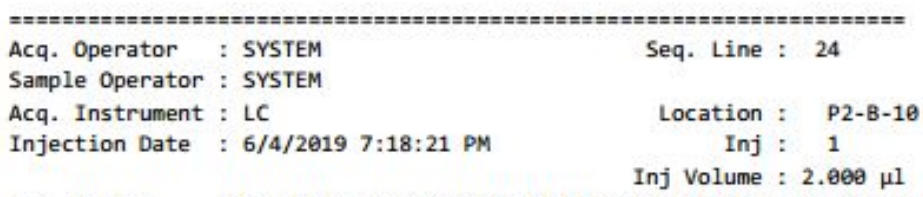

Acq. Method : D:\ChemStation\1\Data\LXX\1XX-2-57\2-57 2019-06-04 12-36-48\1-AD3-98-2-1ML2UL-25C-15MIN. M

Last changed : 5/21/2019 5:17:05 PM by SYSTEM

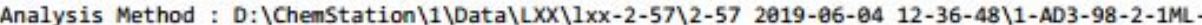
2UL-25C-15MIN.M (Sequence Method)

Last changed : 7/5/2019 3:00:21 PM by SYSTEM (modified after loading)

Additional Info : Peak(s) manually integrated

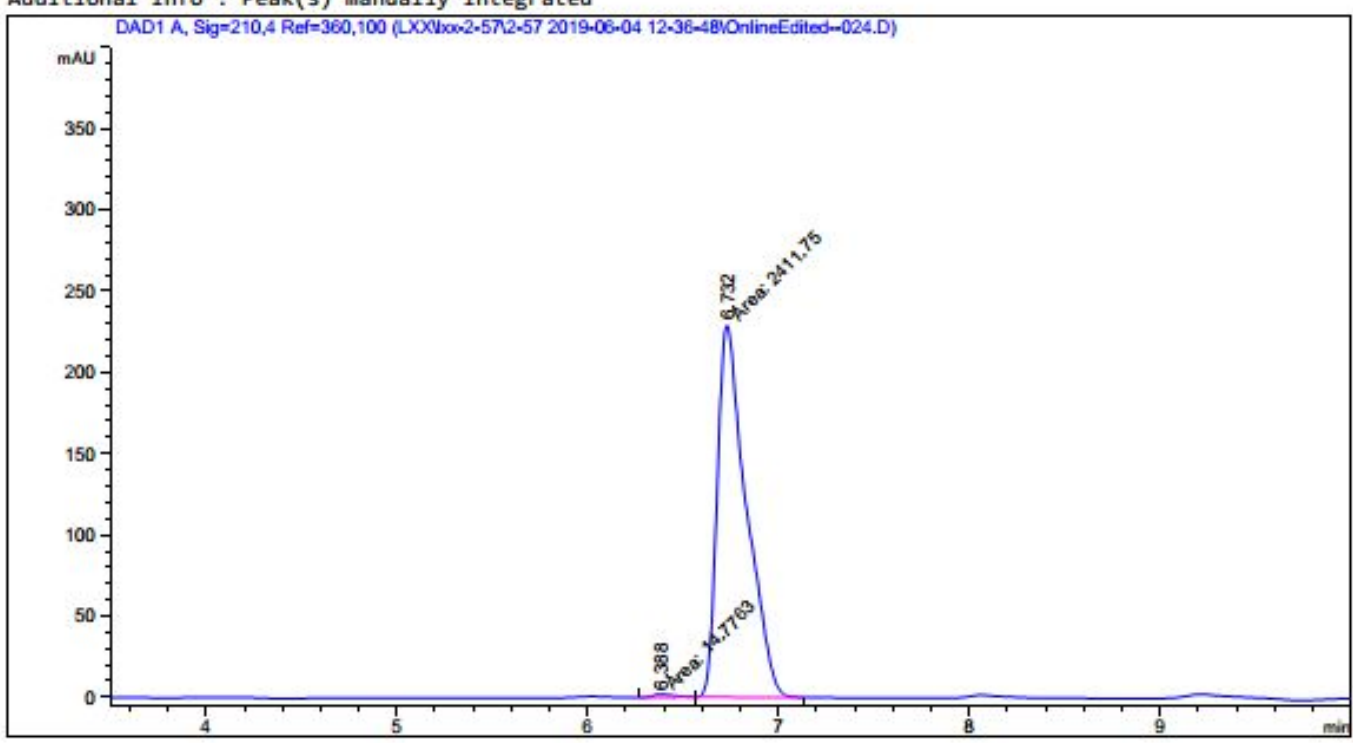

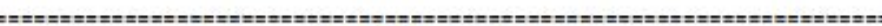

Area Percent Report

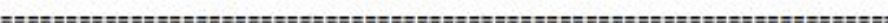

$\begin{array}{lll}\text { Sorted By } & : & \text { Signal } \\ \text { Multiplier } & : & 1.0000 \\ \text { Dilution } & : & 1.0000\end{array}$

Do not use Multiplier \& Dilution Factor with ISTDs

Signal 1: DAD1 A, Sig=210,4 Ref=360, 100

\begin{tabular}{|c|c|c|c|c|c|c|}
\hline $\begin{array}{c}\text { Peak } \\
\#\end{array}$ & $\begin{array}{c}\text { RetTime } \\
\text { [min] }\end{array}$ & Type & $\begin{array}{l}\text { Width } \\
\text { [min] }\end{array}$ & $\begin{array}{c}\text { Area } \\
{\left[\mathrm{mAU}^{*} \mathrm{~s}\right]}\end{array}$ & $\begin{array}{l}\text { Height } \\
\text { [MAU] }\end{array}$ & $\begin{array}{c}\text { Area } \\
\%\end{array}$ \\
\hline$\cdots-1$ & & & & . & - & 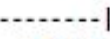 \\
\hline 1 & 6.388 & $\mathrm{MM}$ & 0.1333 & 14.77626 & 1.84762 & 0.6989 \\
\hline 2 & 6.732 & $\mathrm{MM}$ & 0.1754 & 2411.75342 & 229.13083 & 99.3911 \\
\hline$t$ & & & & 2426.52968 & 230.97845 & \\
\hline
\end{tabular}


<smiles>CCC1(C2CCCCC2)C=CC(=O)CC1</smiles>

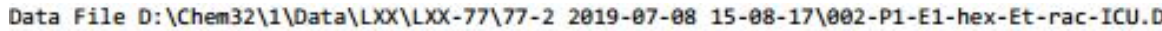
Sample Name: hex-Et-rac-ICU

\begin{tabular}{|c|c|c|c|}
\hline Acq. Operator & : SYSTEM & Seq. Line & 2 \\
\hline Acq. Instrument & : $1290-D A D$ & Location & P1-E-01 \\
\hline Injection Date & : 7/8/2019 3:16:51 PM & $\begin{array}{r}\text { Inj } \\
\text { Inj Volume }\end{array}$ & $\begin{array}{l}1 \\
. \theta 60 \mu 1\end{array}$ \\
\hline
\end{tabular}

Acq. Method : D:\Chem32\1\Data\LXX\LXX-77\77-2 2019-07-08 15-08-17\98-2-0.6-1UL-20MIN.M Last changed : 7/8/2019 3:33:53 PM by SYSTEM (modified after loading)

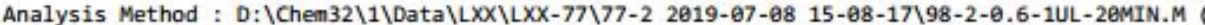
Sequence Method)

Last changed : 7/11/2019 4:10:57 PM by SYSTEM

(modified after loading)

Additional Info: Peak(s) manually integrated

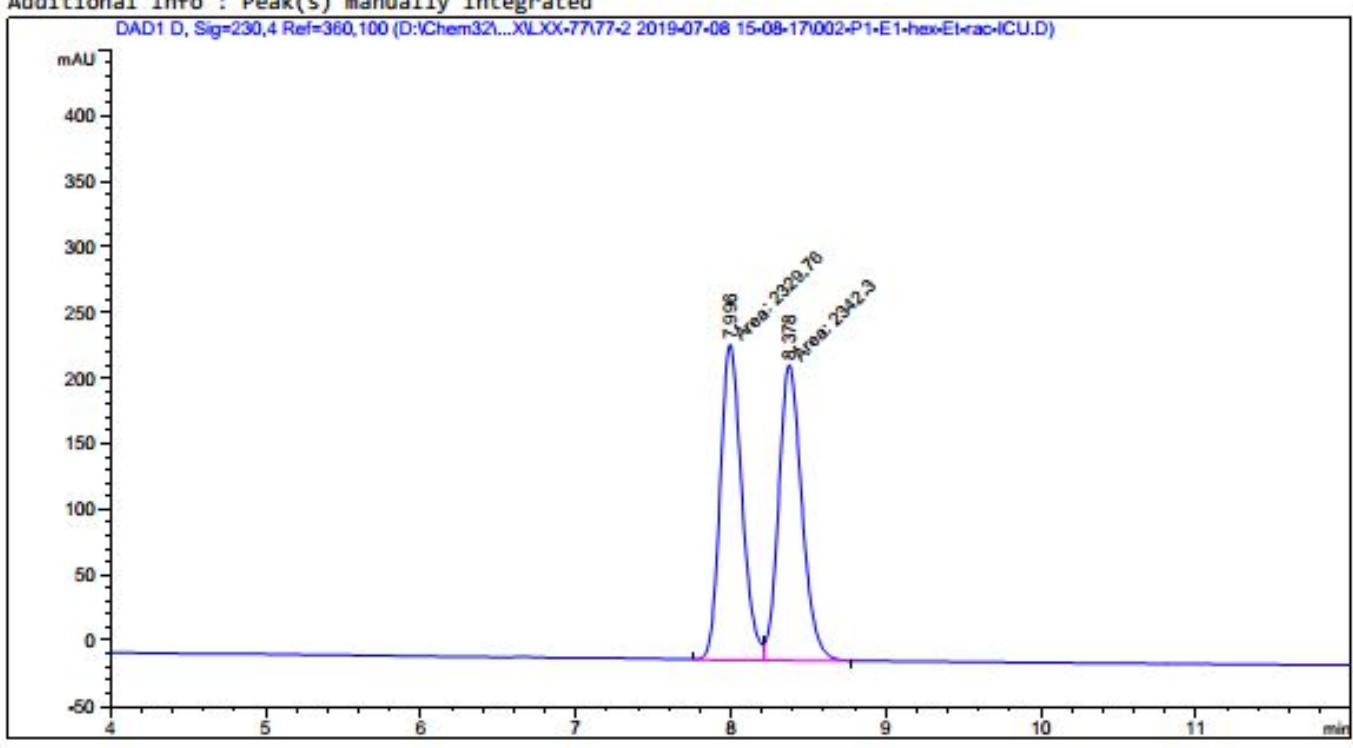

Area Percent Report

$\begin{array}{lll}\text { Sorted By } & : & \text { Signal } \\ \text { Multiplier } & : & 1.0000 \\ \text { Dilution } & : & 1.0000\end{array}$

Do not use Multiplier \& Dilution Factor with ISTDs

Signal 1: DAD1 D, Sig $=230,4$ Ref $=360,100$

\begin{tabular}{llllll}
$\begin{array}{c}\text { Peak RetTime Type } \\
\text { [min] }\end{array}$ & $\begin{array}{c}\text { Width } \\
\text { [min] }\end{array}$ & $\begin{array}{c}\text { Area } \\
\text { [mAU*s] }\end{array}$ & $\begin{array}{c}\text { Height } \\
\text { [mAU] }\end{array}$ & $\begin{array}{c}\text { Area } \\
\%\end{array}$ \\
\hline 1 & $7.996 \mathrm{MF}$ & 0.1615 & 2329.76343 & 240.49940 & 49.8658 \\
2 & $8.378 \mathrm{FM}$ & 0.1734 & 2342.36420 & 225.14246 & 50.1342 \\
& & & & & \\
Totals : & & 4672.06763 & 465.64186
\end{tabular}




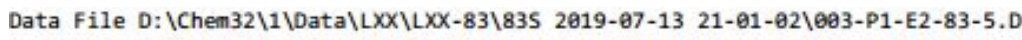

Sample Name: 83-5

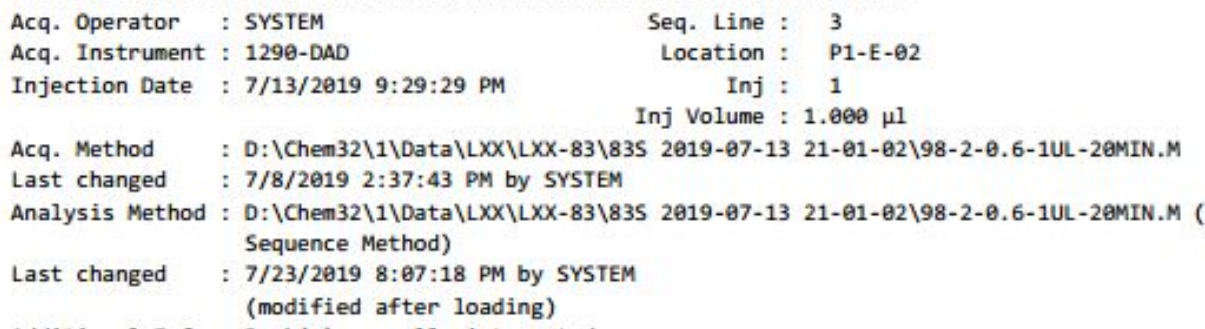

Acq. Method : D:\Chem32\1\Data\LXX\LXX-83\83S 2019-07-13 21-01-02\98-2-0.6-1UL-20MIN.M Last changed : 7/8/2019 2:37:43 PM by SYSTEM

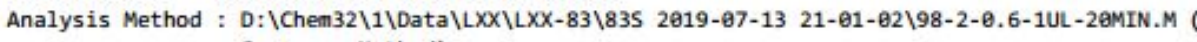
Sequence Method)

Last changed : 7/23/2019 8:67:18 PM by SYSTEM (modified after loading)

Additional Info: Peak(s) manually integrated
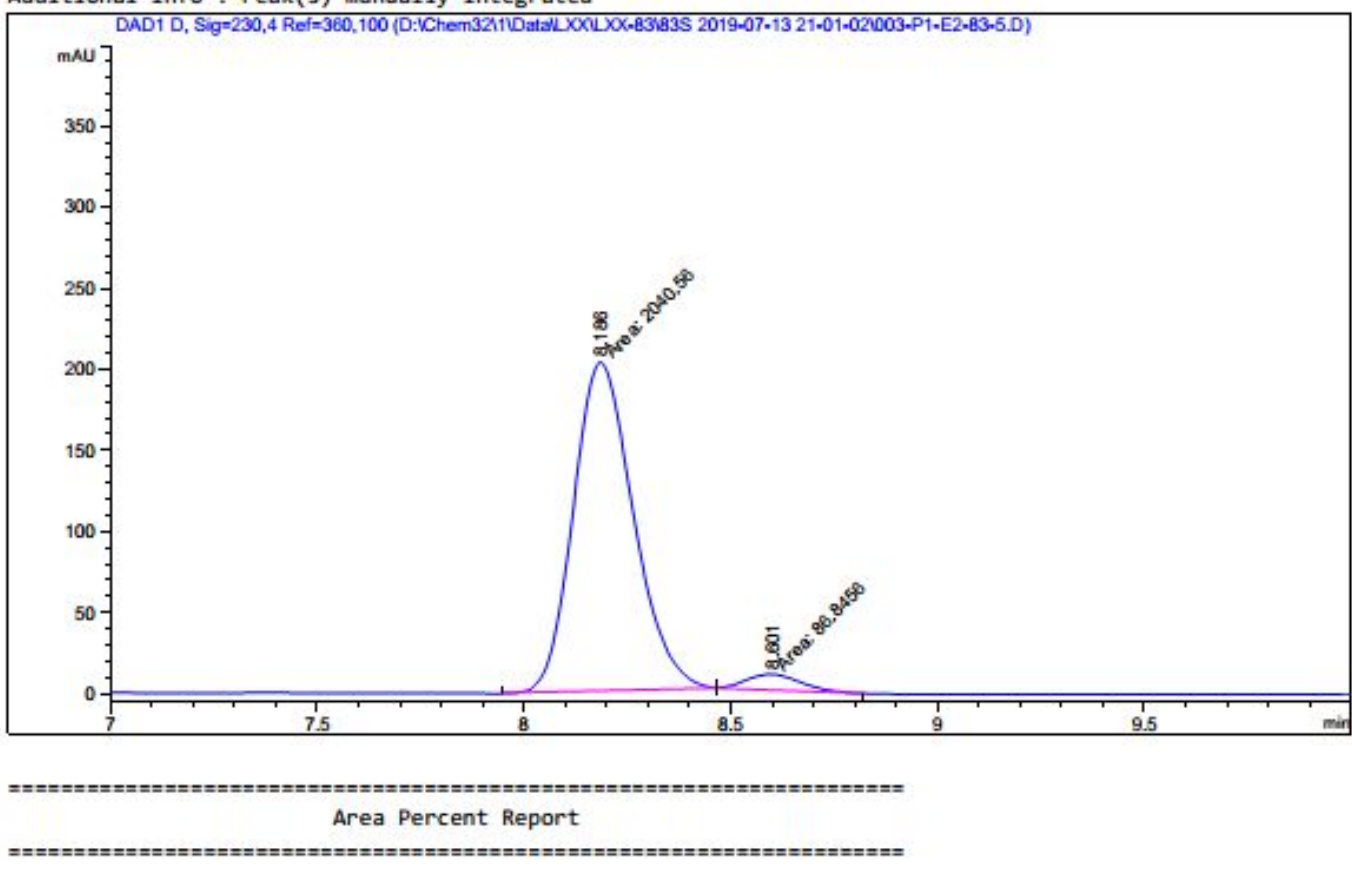

$\begin{array}{lll}\text { Sorted By } & : & \text { Signal } \\ \text { Multiplier } & : & 1.0000 \\ \text { Dilution } & : & 1.0000\end{array}$

Do not use Multiplier \& Dilution Factor with ISTDs

Signal 1: DAD1 D, $\operatorname{Sig}=230,4$ Ref $=360,100$

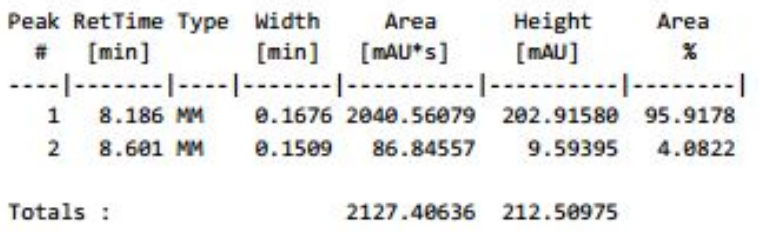




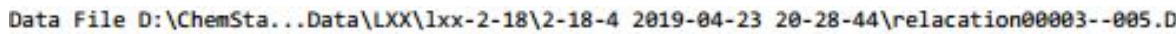
Sample Name: 18-4-Rac
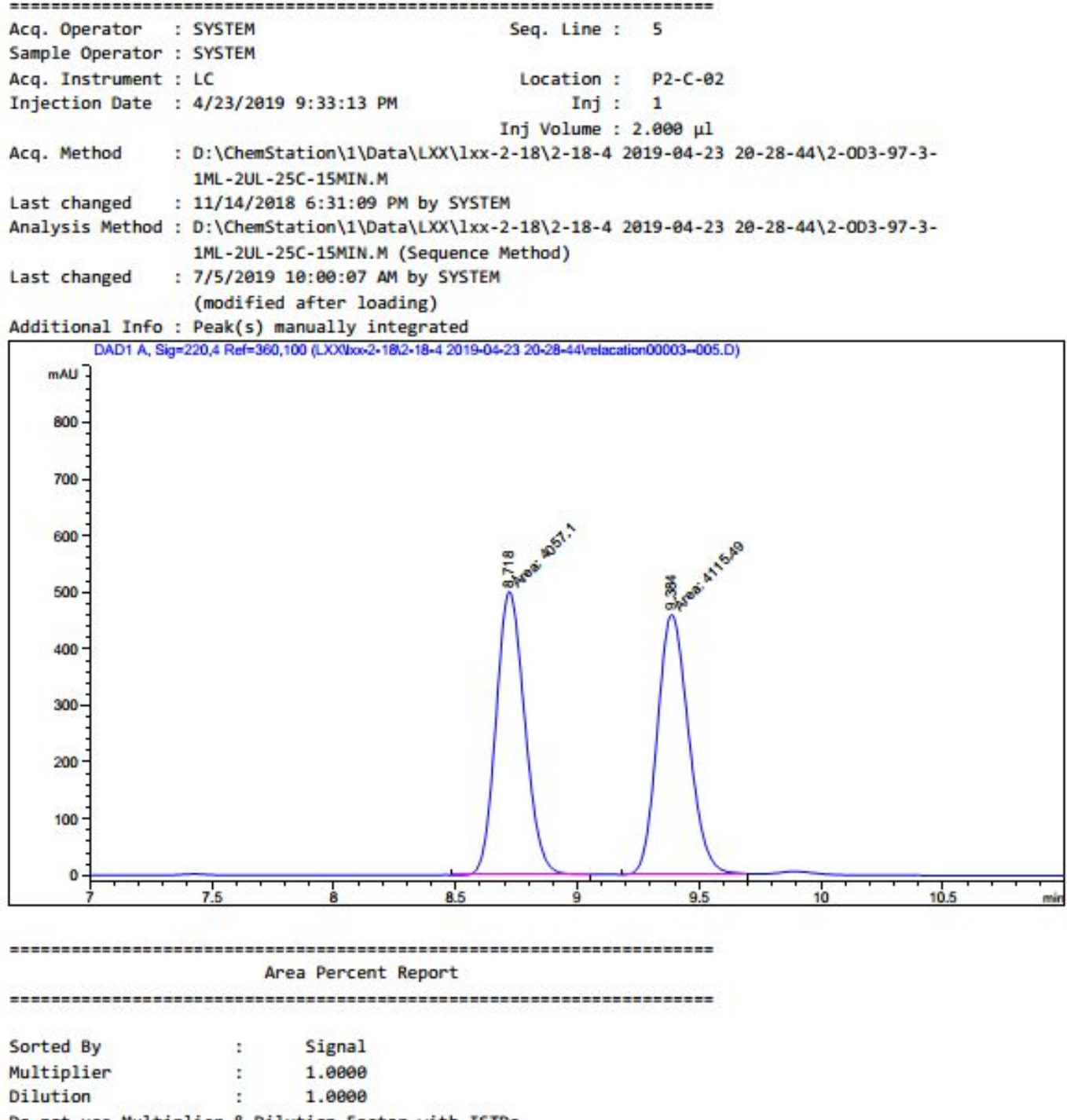

Do not use Multiplier \& Dilution Factor with ISTDs

Signal 1: DAD1 A, $\operatorname{Sig}=220,4$ Ref $=360,100$

\begin{tabular}{cccccc}
$\begin{array}{c}\text { Peak RetTime Type } \\
\text { [min] }\end{array}$ & $\begin{array}{c}\text { Width } \\
\text { [min] }\end{array}$ & $\begin{array}{c}\text { Area } \\
\text { [mAU*s] }\end{array}$ & $\begin{array}{l}\text { Height } \\
\text { [mAU] }\end{array}$ & $\begin{array}{c}\text { Area } \\
\%\end{array}$ \\
\hline 1 & $8.718 \mathrm{MM}$ & 0.1354 & 4657.09912 & 499.29556 & 49.6428 \\
2 & $9.384 \mathrm{MF}$ & 0.1491 & 4115.48828 & 460.13290 & 50.3572 \\
& & & & & \\
Totals : & & 8172.58740 & 959.42847
\end{tabular}




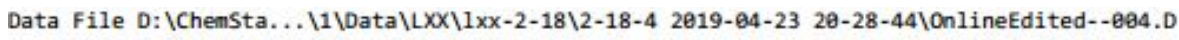
Sample Name: $18-4$

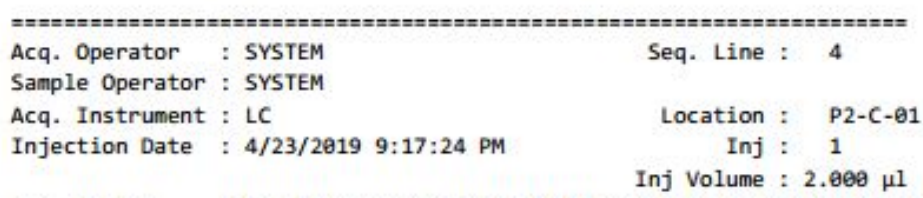

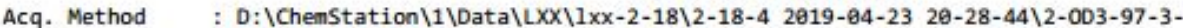
1 ML-2UL-25C-15MIN. M

Last changed : 11/14/2018 6:31:e9 PM by SYSTEM

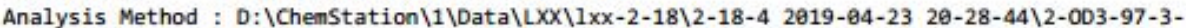
1ML-2UL-25C-15MIN.M (Sequence Method)

Last changed : 7/5/2019 9:57:45 AM by SYSTEM

(modified after loading)

Additional Info: Peak(s) manually integrated

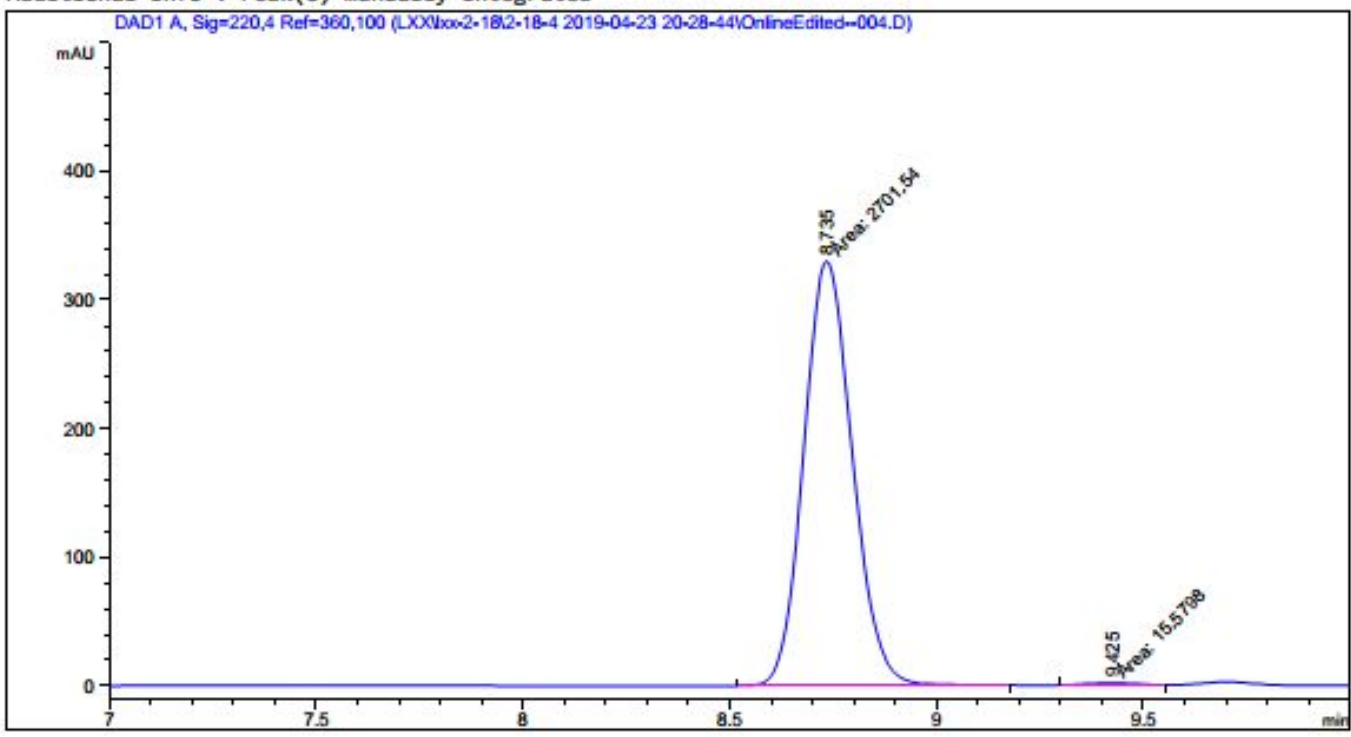

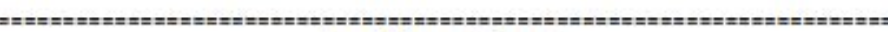

Area Percent Report

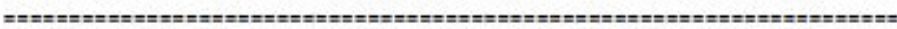

Sorted By : : Signal

Multiplier : : $1 . \theta 00 \theta$

Dilution : 1.0000

Do not use Multiplier \& Dilution Factor with ISTDs

Signal 1: DAD1 A, Sig $=220,4$ Ref $=360,100$

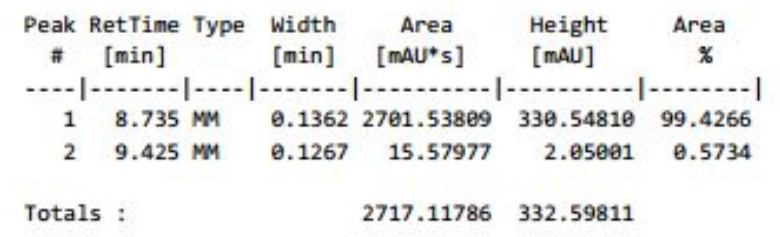

LC 7/5/2019 9:58:10 AM SYSTEM

Page 1 of 2 
<smiles>O=C1C=C[C@]2(CC1)CCc1cc(F)ccc12</smiles>

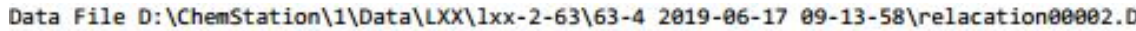
Sample Name: p-F-five-Rac

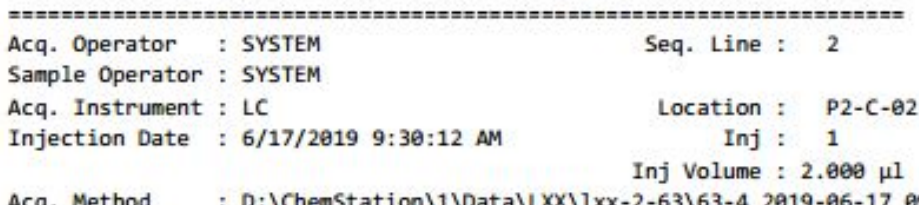

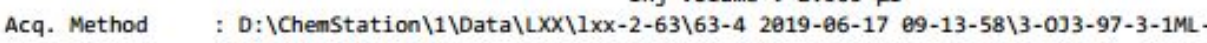
2UL-25C-3OMIN. M

Last changed : 1/4/2019 7:42:30 PM by SYSTEM

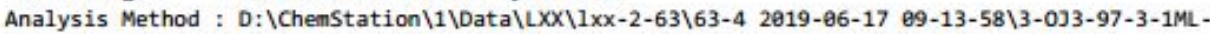
2UL-25C-3eMIN.M (Sequence Method)

Last changed : 7/5/2019 3:33:35 PM by SYSTEM (modified after loading)

Additional Info : Peak(s) manually integrated

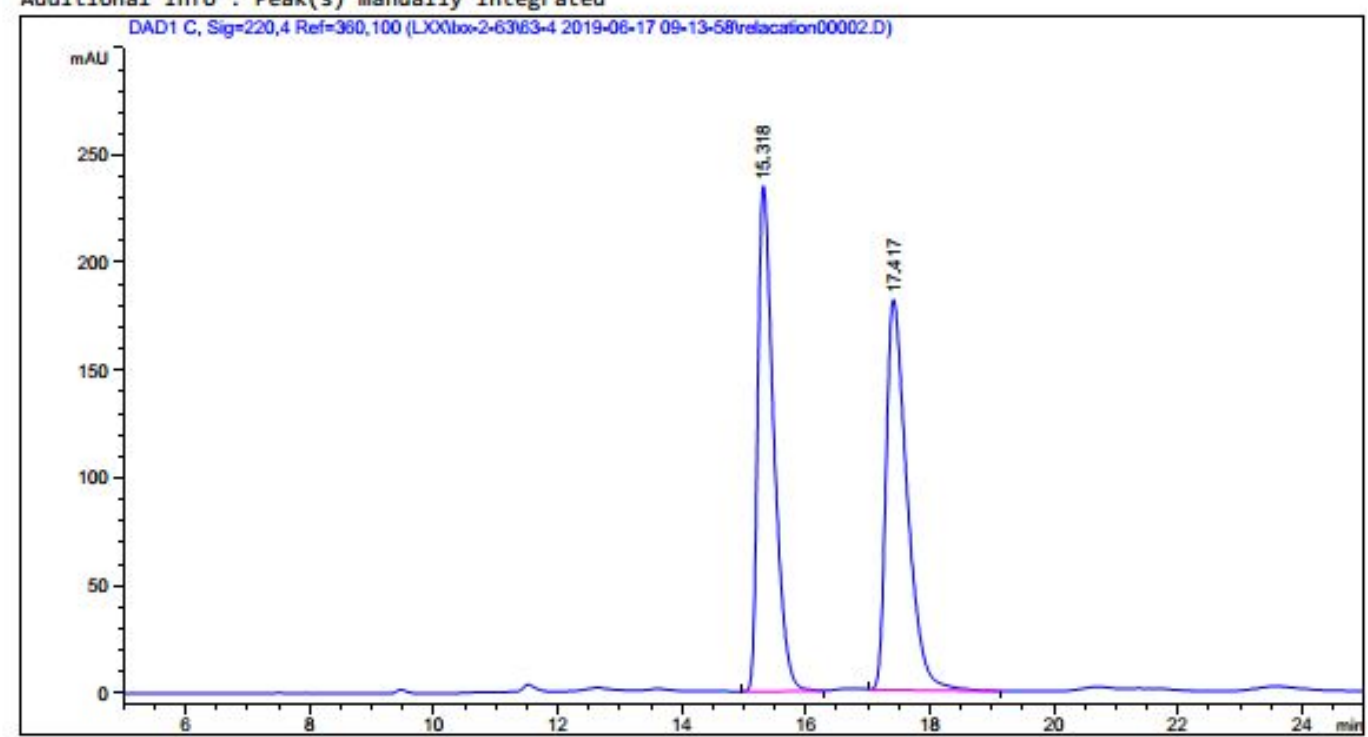

\section{Area Percent Report}

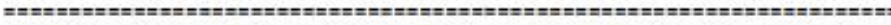

$\begin{array}{lll}\text { Sorted By } & : & \text { Signal } \\ \text { Multiplier } & : & 1.0000 \\ \text { Dilution } & : & 1.0000\end{array}$

Do not use Multiplier \& Dilution Factor with ISTDs

Signal 1: DAD1 C, Sig=220,4 Ref $=360,100$

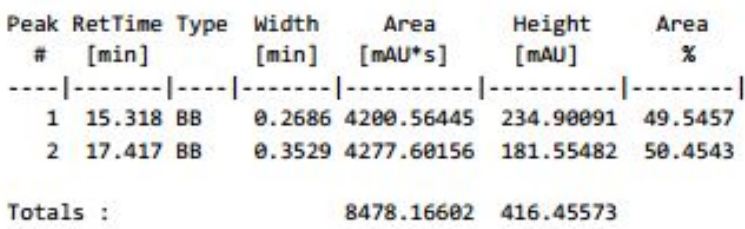




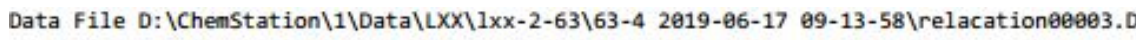
Sample Name: 63-4

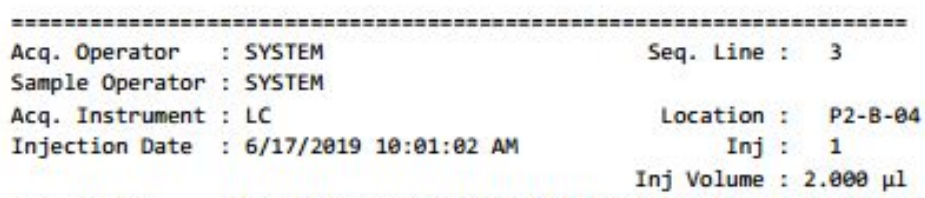

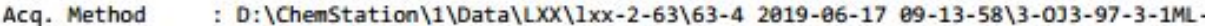
2UL-25C-30MIN. M

Last changed : 1/4/2019 7:42:30 PM by SYSTEM

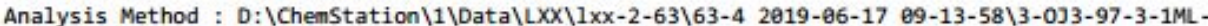
2UL-25C-30MIN.M (Sequence Method)

Last changed : 7/5/2019 3:33:35 PM by SYSTEM

(modified after loading)

Additional Info : Peak(s) manually integrated

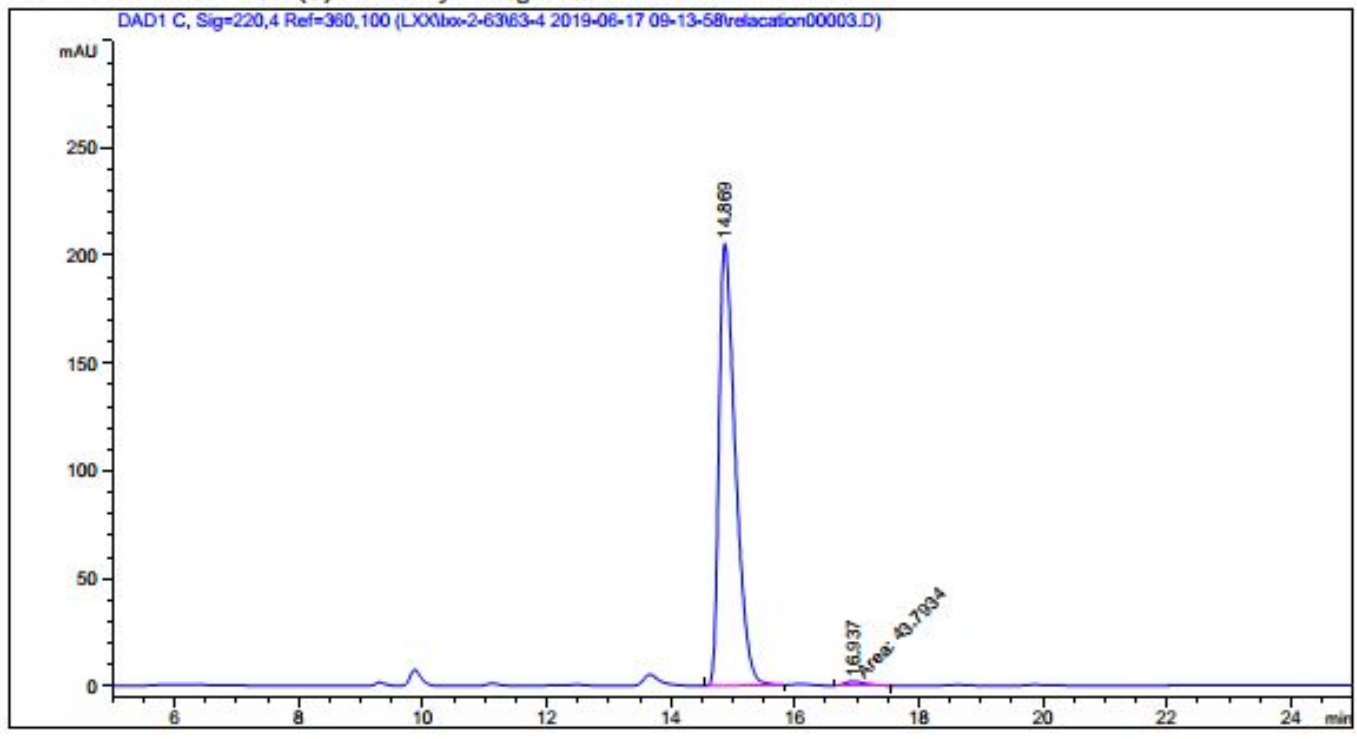

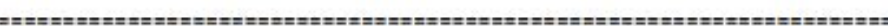

Area Percent Report

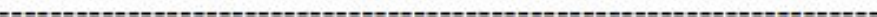

\section{Sorted By : : Signal}

Multiplier : $\quad 1.00 \theta$

Dilution : 1.0000

Do not use Multiplier \& Dilution Factor with ISTDs

Signal 1: DAD1 C, $\operatorname{Sig}=220,4$ Ref $=360,100$

\begin{tabular}{|c|c|c|c|c|c|}
\hline $\begin{array}{c}\text { Peak } \\
\#\end{array}$ & $\begin{array}{l}\text { RetTime Type } \\
\text { [min] }\end{array}$ & $\begin{array}{l}\text { Width } \\
\text { [min] }\end{array}$ & $\begin{array}{c}\text { Area } \\
{[\mathrm{mAU} * \mathrm{~s}]}\end{array}$ & $\begin{array}{l}\text { Height } \\
\text { [mAU] }\end{array}$ & $\begin{array}{c}\text { Area } \\
\text { \% }\end{array}$ \\
\hline$\ldots$ & | & $\ldots$ & | & - n. & $\ldots$ \\
\hline 1 & $14.869 \mathrm{BB}$ & 0.2758 & 3766.81201 & 205.58919 & 98.8508 \\
\hline 2 & $16.937 \mathrm{mM}$ & 0.3648 & 43.79337 & 2.00065 & 1.1492 \\
\hline Tota & 15 & & 3810.60538 & 207.58983 & \\
\hline
\end{tabular}

LC 7/5/2019 3:33:40 PM SYSTEM 
<smiles>O=C1C=C[C@]2(CC1)CCc1cc(Cl)ccc12</smiles>

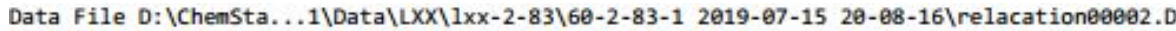
Sample Name: 60-2-RAC
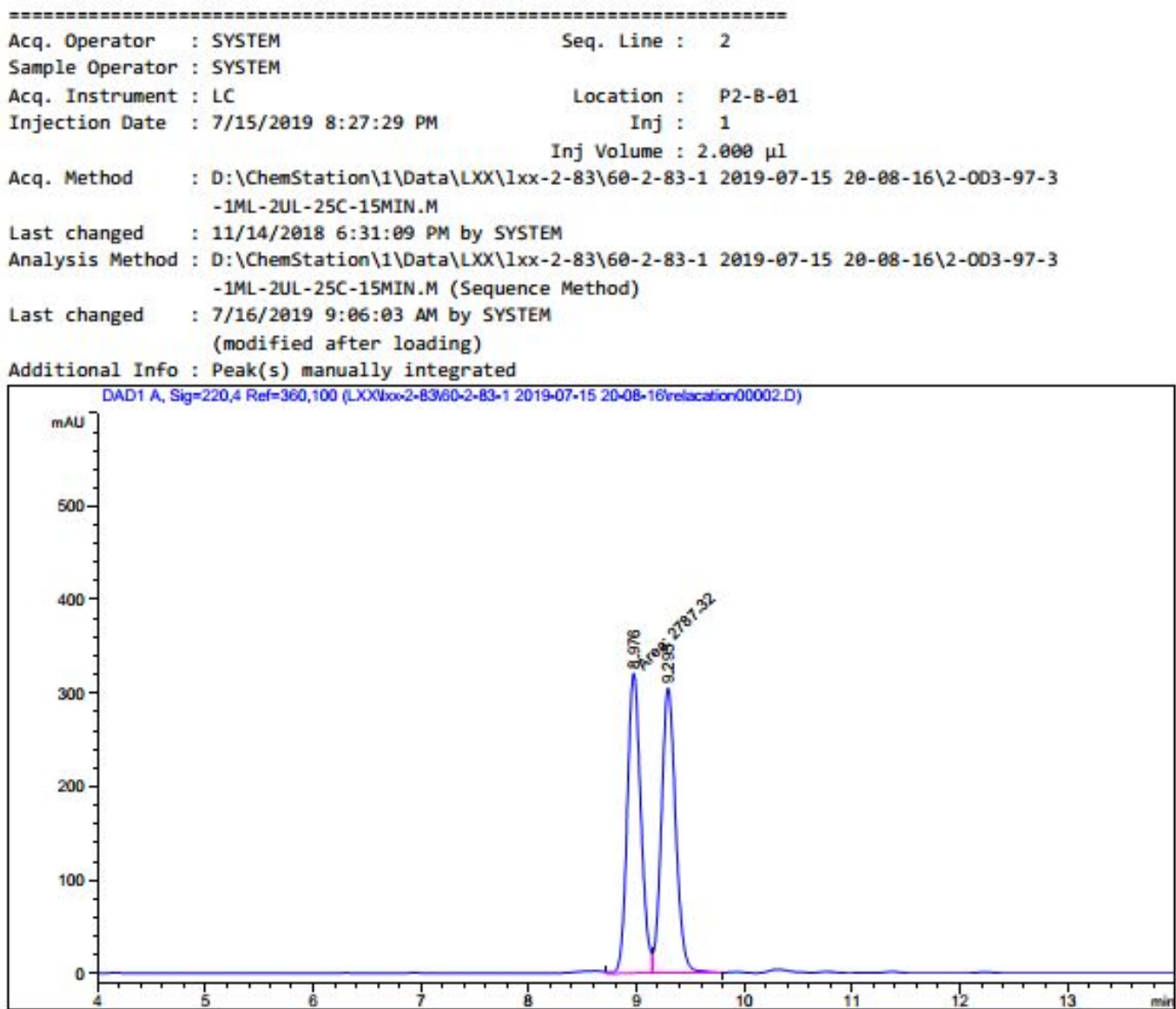

Area Percent Report

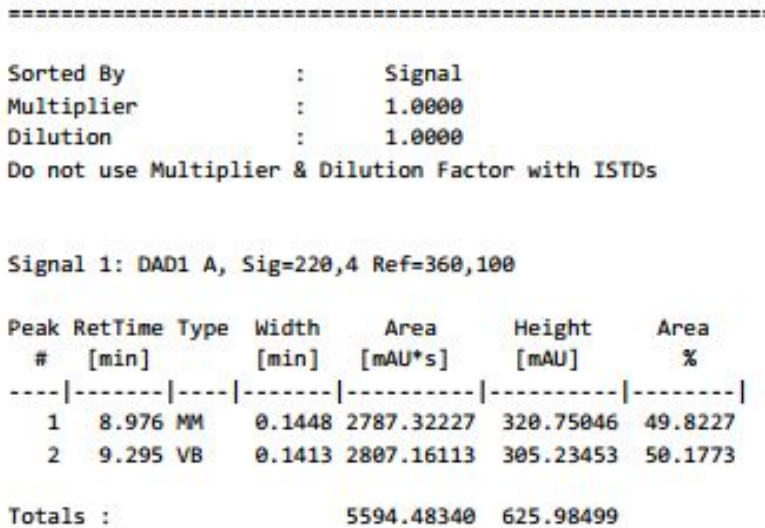




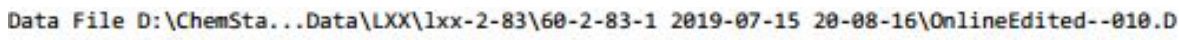
Sample Name: 60-2

\begin{tabular}{|c|c|c|c|}
\hline $\begin{array}{l}\text { Acq. Operator } \\
\text { Sample Operator }\end{array}$ & $\begin{array}{l}\text { : SYSTEM } \\
: \text { SYSTEM }\end{array}$ & Seq. Line & $: 10$ \\
\hline Acq. Instrument & : LC & Location & $\mathrm{P} 2-\mathrm{B}-62$ \\
\hline Injection Date & : 7/15/2019 10:50:28 PM & $\begin{array}{r}\text { Inj } \\
\text { Inj Volume }\end{array}$ & $\begin{array}{l}: \quad 1 \\
: 2.000 \mu 1\end{array}$ \\
\hline
\end{tabular}

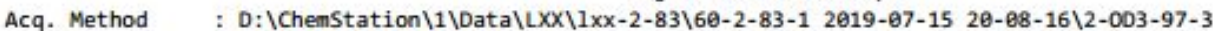
-1 ML-2UL-25C-15MIN.M

Last changed : 11/14/2018 6:31:e9 PM by SYSTEM

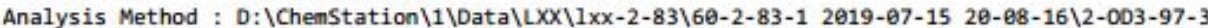

-1ML-2UL-25C-15MIN.M (Sequence Method)

Last changed : 7/16/2019 9:84:26 AM by SYSTEM

(modified after loading)

Additional Info: Peak(s) manually integrated

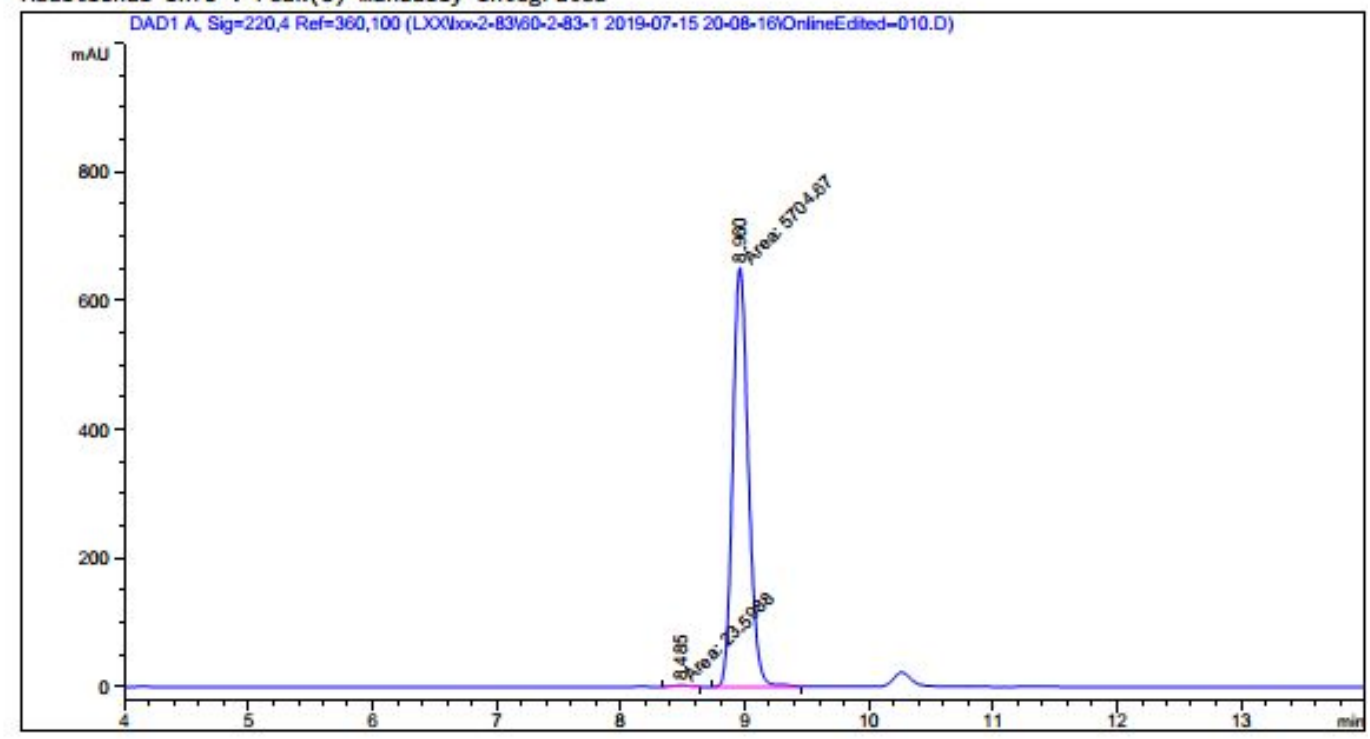

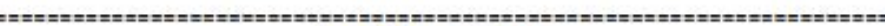

Area Percent Report

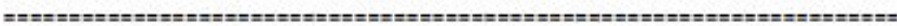

Sorted By : : Signal

Multiplier : : 1.000

Dilution : 1.000

Do not use Multiplier \& Dilution Factor with ISTDs

Signal 1: DAD1 A, Sig $=220,4$ Ref $=360,100$

\begin{tabular}{|c|c|c|c|c|c|c|}
\hline $\begin{array}{c}\text { Peak } \\
\#\end{array}$ & $\begin{array}{c}\text { RetTime } \\
\text { [min] }\end{array}$ & Type & $\begin{array}{l}\text { Width } \\
\text { [min] }\end{array}$ & $\begin{array}{c}\text { Area } \\
{[\mathrm{mAU} * \mathrm{~s}]}\end{array}$ & $\begin{array}{l}\text { Height } \\
\text { [mAU] }\end{array}$ & $\begin{array}{c}\text { Area } \\
\text { \% }\end{array}$ \\
\hline$\ldots-1$ & -..... & $\ldots$ & ......... & | & - & .......... \\
\hline 1 & 8.485 & MM & 0.1407 & 23.59884 & 2.79450 & 0.4120 \\
\hline 2 & 8.960 & MM & 0.1458 & 5784.67334 & 652.08038 & 99.5880 \\
\hline Total & Is : & & & 5728.27218 & 654.87488 & \\
\hline
\end{tabular}

LC 7/16/2019 9:04:36 AM SYSTEM

Page 1 of 2 
<smiles>O=C1C=CC2(CC1)CCc1ccc(Br)cc12</smiles>

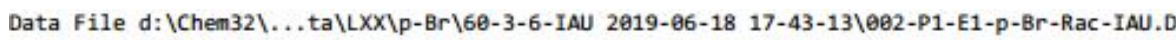
Sample Name: p-Br-Rac-IAU

\begin{tabular}{|c|c|c|c|}
\hline Acq. Operator & : SYSTEM & Seq. Line & 2 \\
\hline Acq. Instrument & : $1290-D A D$ & Location & P1-E-61 \\
\hline Injection Date & : 6/18/2019 5:55:23 PM & $\begin{array}{r}\text { Inj } \\
\text { Inj Volume }\end{array}$ & $\begin{array}{l}: \quad 1 \\
: 1.000 \mu 1\end{array}$ \\
\hline
\end{tabular}

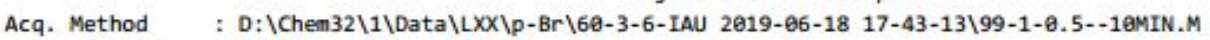
Last changed : 6/18/2019 4:40:45 PM by SYSTEM

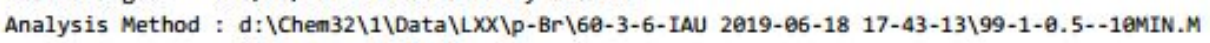
(Sequence Method)

Last changed : 7/5/2019 3:34:41 PM by SYSTEM

(modified after loading)

Additional Info : Peak(s) manually integrated

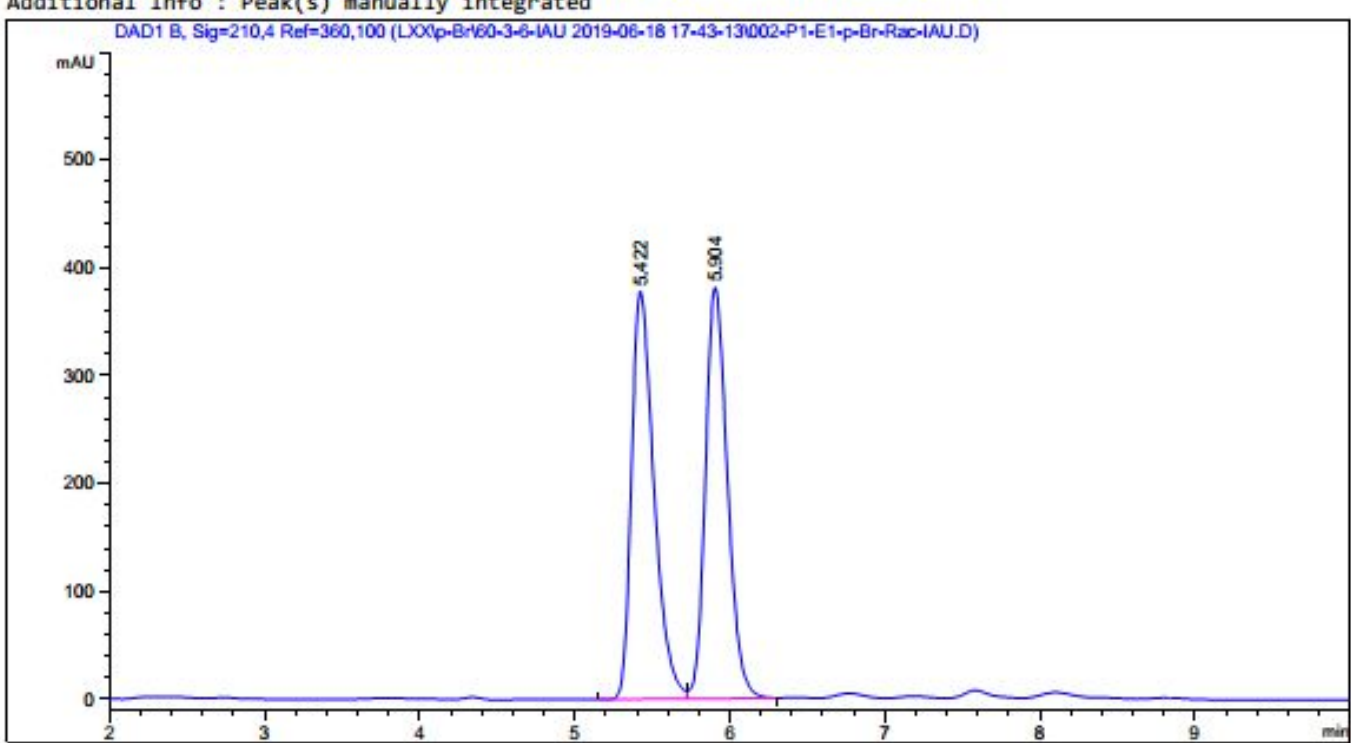

Area Percent Report

$\begin{array}{lll} & & \\ & & \\ \text { Sorted By } & : & \text { Signal } \\ \text { Multiplier } & : & 1.0000 \\ \text { Dilution } & : & 1.0000\end{array}$

Do not use Multiplier $\&$ Dilution Factor with ISTDs

Signal 1: DAD1 B, $\operatorname{Sig}=210,4$ Ref $=360,100$

\begin{tabular}{llllll}
$\begin{array}{l}\text { Peak RetTime Type } \\
\text { [min] }\end{array}$ & $\begin{array}{c}\text { Width } \\
\text { [min] }\end{array}$ & $\begin{array}{c}\text { Area } \\
\text { [mAU*s] }\end{array}$ & $\begin{array}{c}\text { Height } \\
\text { [mAU] }\end{array}$ & $\begin{array}{c}\text { Area } \\
\%\end{array}$ \\
\hline 1 & 5.422 BV & 0.1557 & 3811.63647 & 377.78107 & 50.3845 \\
2 & 5.904 VB & 0.1530 & 3753.45825 & 380.85379 & 49.6155 \\
& & & & & \\
Totals : & & 7565.09473 & 758.63486
\end{tabular}




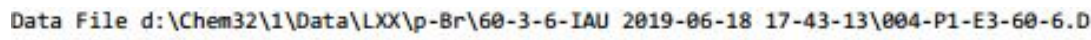
Sample Name: 60-6
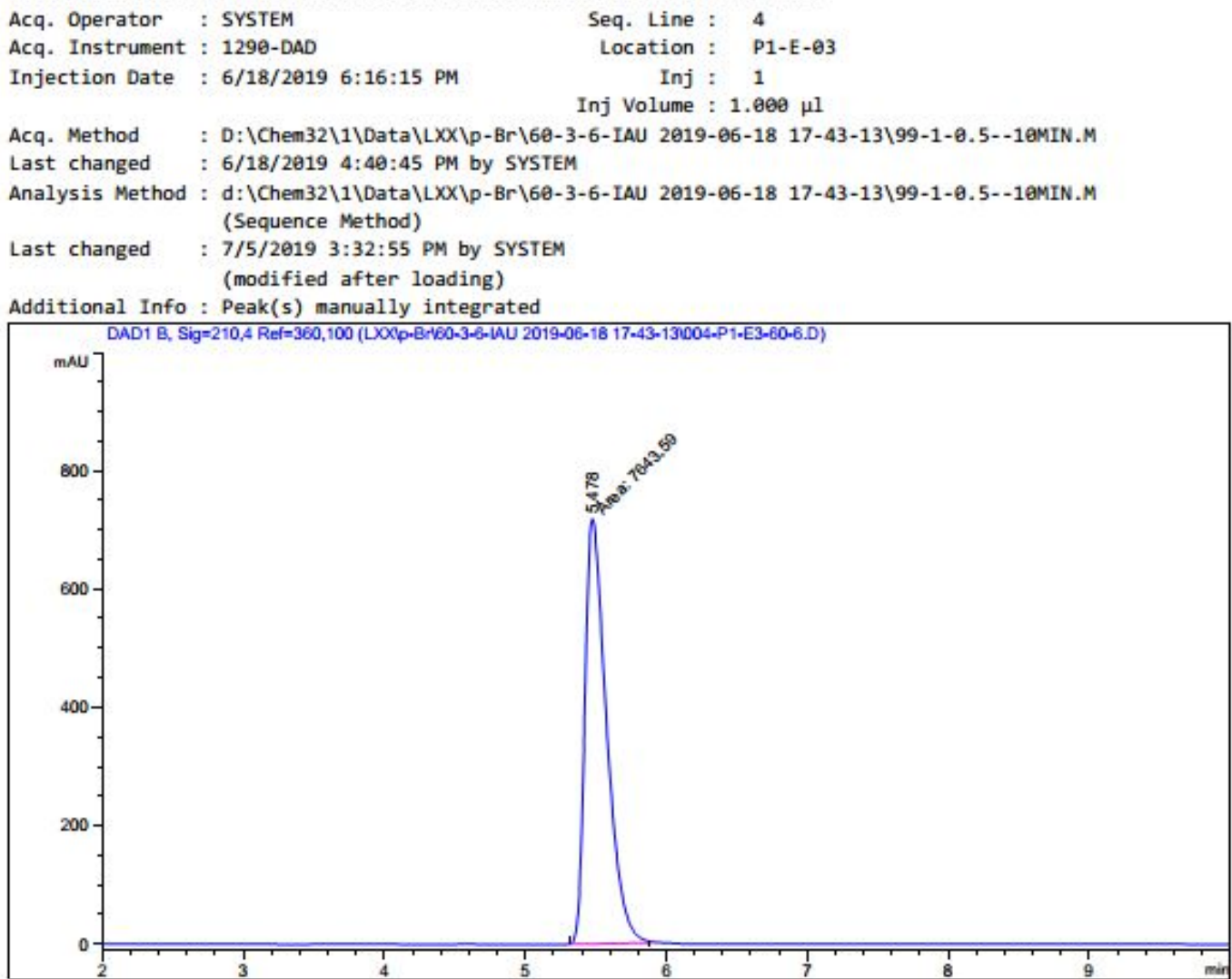

Area Percent Report

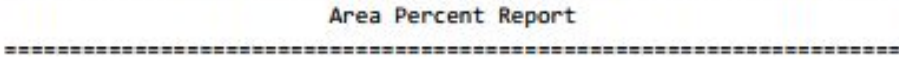

$\begin{array}{lll}\text { Sorted By } & : & \text { Signal } \\ \text { Multiplier } & : & 1.00 \theta 0\end{array}$

Dilution : 1.0898

Do not use Multiplier \& Dilution Factor with ISTDs

Signal 1: DAD1 B, Sig $=210,4$ Ref $=360,100$

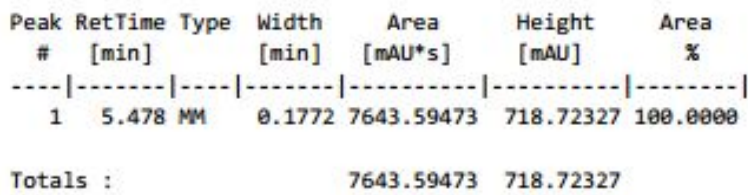


<smiles>COc1cccc2c1CC[C@@]21C=CC(=O)CC1</smiles>

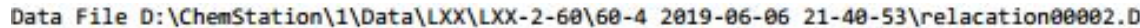
Sample Name: 60-4-RAC

\begin{tabular}{|c|c|c|c|}
\hline $\begin{array}{l}\text { Acq. Operator } \\
\text { Sample Operator }\end{array}$ & $\begin{array}{l}\text { : SYSTEM } \\
\text { : SYSTEM }\end{array}$ & Seq. Line & 2 \\
\hline Acq. Instrument & : LC & Location & P2 $-C-64$ \\
\hline Injection Date & : 6/6/2019 9:58:37 PM & $\begin{array}{r}\text { Inj } \\
\text { Inj Volume }\end{array}$ & $\frac{1}{2.000 \mu 1}$ \\
\hline
\end{tabular}

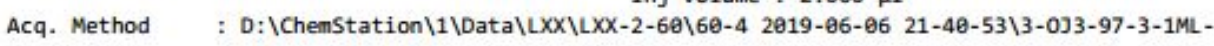
2UL - 25C-3EMIN. M

Last changed : 1/4/2019 7:42:30 PM by SYSTEM

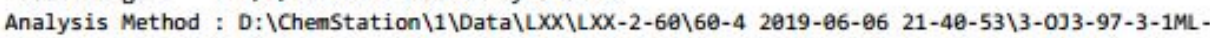

2UL-25C-30MIN.M (Sequence Method)

Last changed : 7/12/2019 $8: 31: 24$ PM by SYSTEM

(modified after loading)

Additional Info: Peak(s) manually integrated

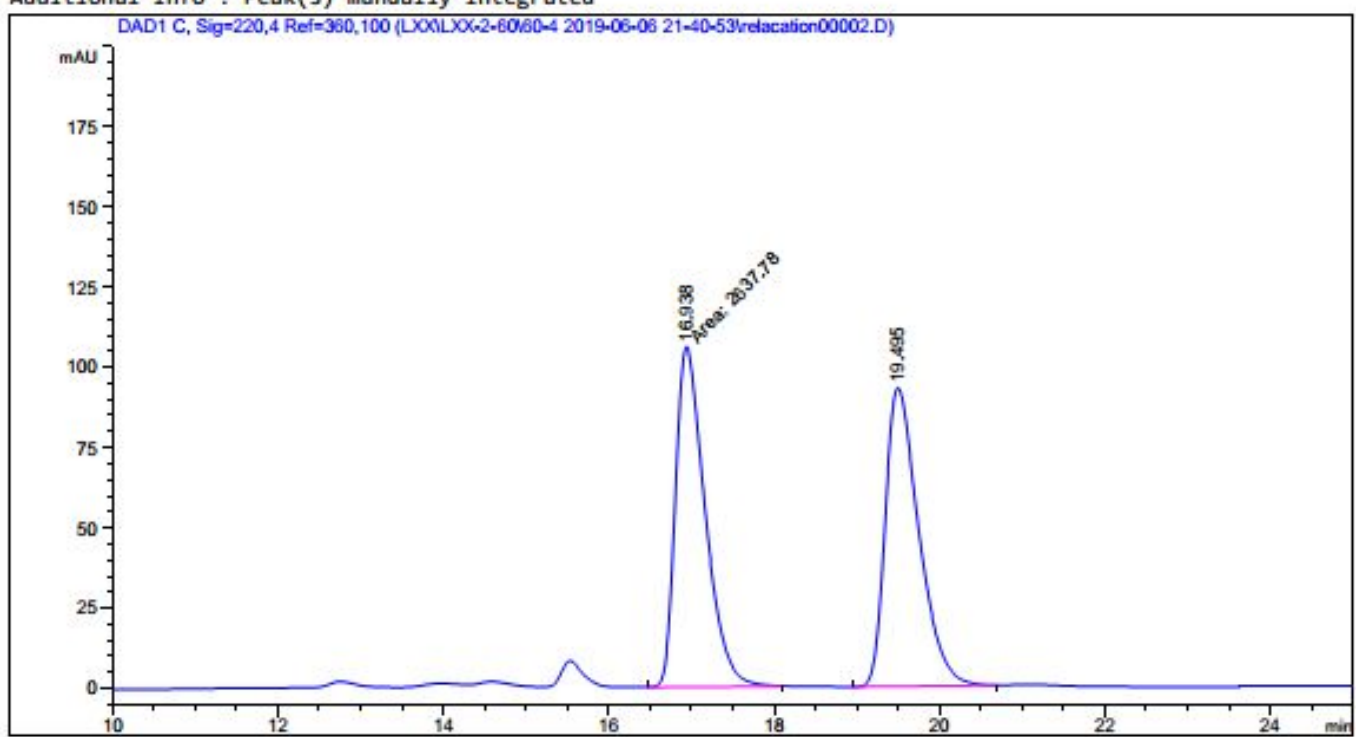

Area Percent Report

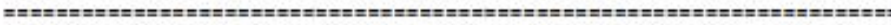

Sorted By : : Signal

Multiplier : : 1.000

Dilution : 1.000

Do not use Multiplier \& Dilution Factor with ISTDs

Signal 1: DAD1 C, Sig $=220,4$ Ref $=360,100$

\begin{tabular}{|c|c|c|c|c|c|}
\hline $\begin{array}{c}\text { Peak } \\
\#\end{array}$ & $\begin{array}{l}\text { RetTime Type } \\
\text { [min] }\end{array}$ & $\begin{array}{l}\text { Width } \\
\text { [min] }\end{array}$ & $\begin{array}{c}\text { Area } \\
{\left[\mathrm{mAU}^{*} \mathrm{~s}\right]}\end{array}$ & $\begin{array}{l}\text { Height } \\
\text { [maU] }\end{array}$ & $\begin{array}{c}\text { Area } \\
\%\end{array}$ \\
\hline 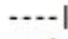 & .......... & .......... & | & . & 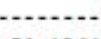 \\
\hline 1 & $16.938 \mathrm{MM}$ & 0.4145 & 2637.78027 & 106.05884 & 50.1364 \\
\hline 2 & $19.495 \mathrm{BB}$ & 0.4293 & 2623.42383 & 92.95547 & 49.8636 \\
\hline Tot & : & & $5261.2841 \theta$ & 199.01431 & \\
\hline
\end{tabular}




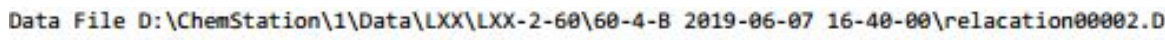
Sample Name: $60-4$

\begin{tabular}{|c|c|c|c|}
\hline $\begin{array}{l}\text { Acq. Operator } \\
\text { Sample Operator }\end{array}$ & $\begin{array}{l}: \text { SYSTEM } \\
: \text { SYSTEM }\end{array}$ & Seq. Line & : \\
\hline Acq. Instrument & : LC & Location & P2-B-64 \\
\hline Injection Date & : 6/7/2019 4:57:59 PM & Inj & : \\
\hline
\end{tabular}

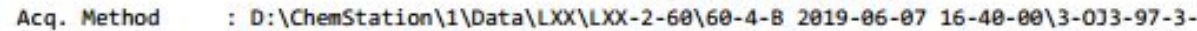
1ML-2UL-25C-30MIN.M

Last changed : $1 / 4 / 2019$ 7:42:30 PM by SYSTEM

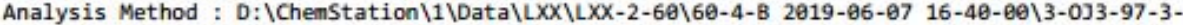
1ML-2UL-25C-30MIN.M (Sequence Method)

Last changed : 7/12/2019 8:25:56 PM by SYSTEM

(modified after loading)

Additional Info: Peak(s) manually integrated

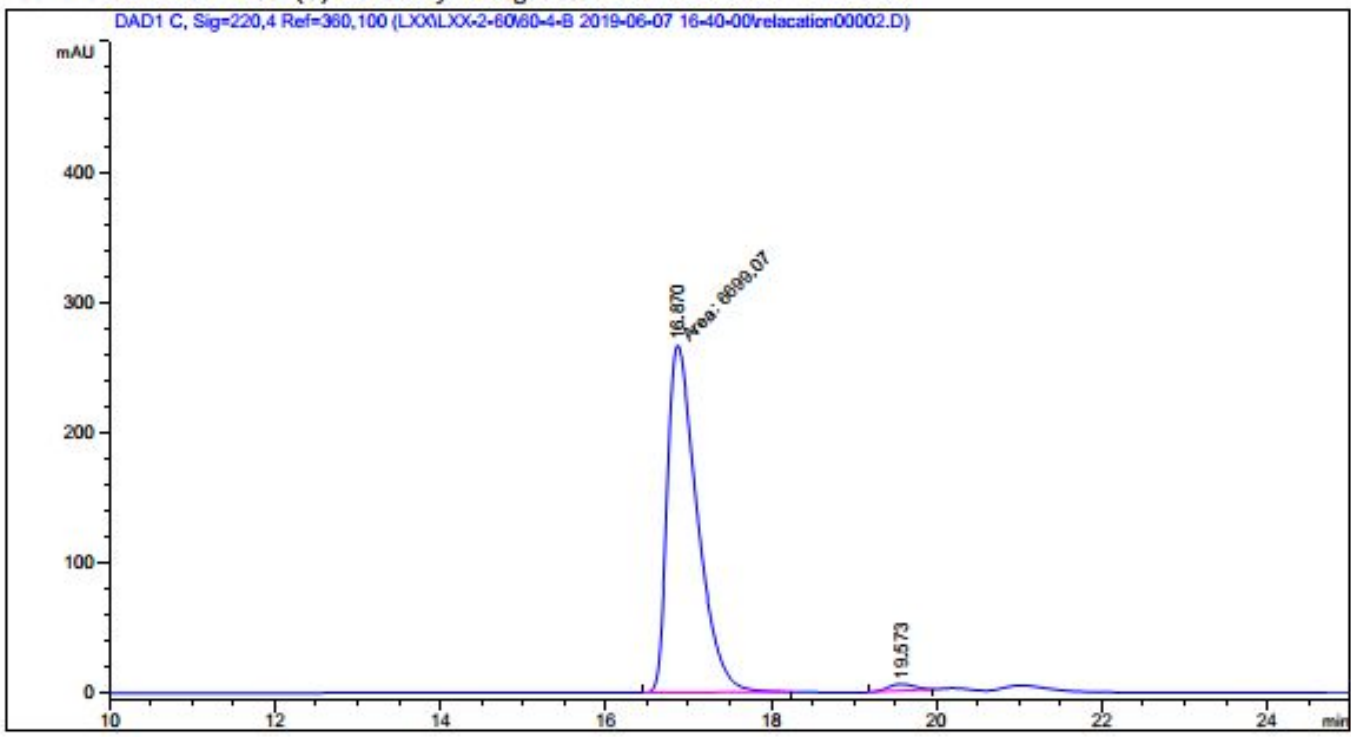

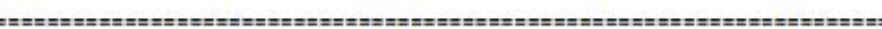

Area Percent Report

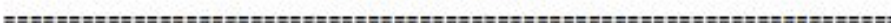

$\begin{array}{lll}\text { Sorted By } & : & \text { Signal } \\ \text { Multiplier } & : & 1.0000 \\ \text { Dilution } & : & 1.0000\end{array}$

Do not use Multiplier \& Dilution Factor with ISTDs

Signal 1: DAD1 C, Sig $=220,4$ Ref $=360,100$

\begin{tabular}{|c|c|c|c|c|c|c|}
\hline $\begin{array}{c}\text { Peak } \\
\#\end{array}$ & $\begin{array}{l}\text { RetTime } \\
\text { [min] }\end{array}$ & Type & $\begin{array}{l}\text { Width } \\
\text { [min] }\end{array}$ & $\begin{array}{c}\text { Area } \\
{\left[\mathrm{mAU}^{*} \mathrm{~s}\right]}\end{array}$ & $\begin{array}{l}\text { Height } \\
\text { [mAU] }\end{array}$ & $\begin{array}{c}\text { Area } \\
\%\end{array}$ \\
\hline$\ldots-1$ & $\ldots$ & & ......... & | & $\ldots$ & - \\
\hline 1 & 16.870 & MM & 0.4190 & 6699.07178 & 266.47314 & 98.3926 \\
\hline 2 & 19.573 & $B B$ & 0.3463 & 109.44316 & 4.98155 & 1.6074 \\
\hline Tot & & & & 6808.51494 & 271.45470 & \\
\hline
\end{tabular}

LC 7/12/2019 8:26:01 PM SYSTEM 
<smiles>COc1cc2c(c(OC)c1)[C@@]1(C=CC(=O)CC1)CC2</smiles>

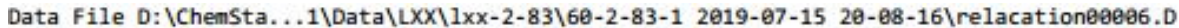
Sample Name: 83-1-rac

\begin{tabular}{|c|c|c|c|}
\hline $\begin{array}{l}\text { Acq. Operator } \\
\text { Sample Operator }\end{array}$ & $\begin{array}{l}\text { : SYSTEM } \\
: \text { SYSTEM }\end{array}$ & Seq. Line : & 6 \\
\hline Acq. Instrument & : LC & Location : & P2-B-03 \\
\hline Injection Date & : 7/15/2019 9:28:00 PM & $\begin{array}{r}\text { Inj : } \\
\text { Inj Volume : }\end{array}$ & $\begin{array}{l}: \quad 1 \\
: \quad 2.009 \mu 1\end{array}$ \\
\hline
\end{tabular}

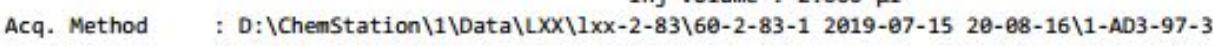
-1 ML-2UL-25C-25MIN.M

Last changed : 11/28/2018 10:55:29 AM by SYSTEM

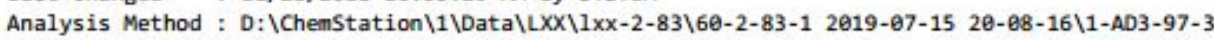
-1ML-2UL-25C-25MIN.M (Sequence Method)

Last changed : 7/16/2019 9:10:59 AM by SYSTEM

(modified after loading)

Additional Info : Peak(s) manually integrated

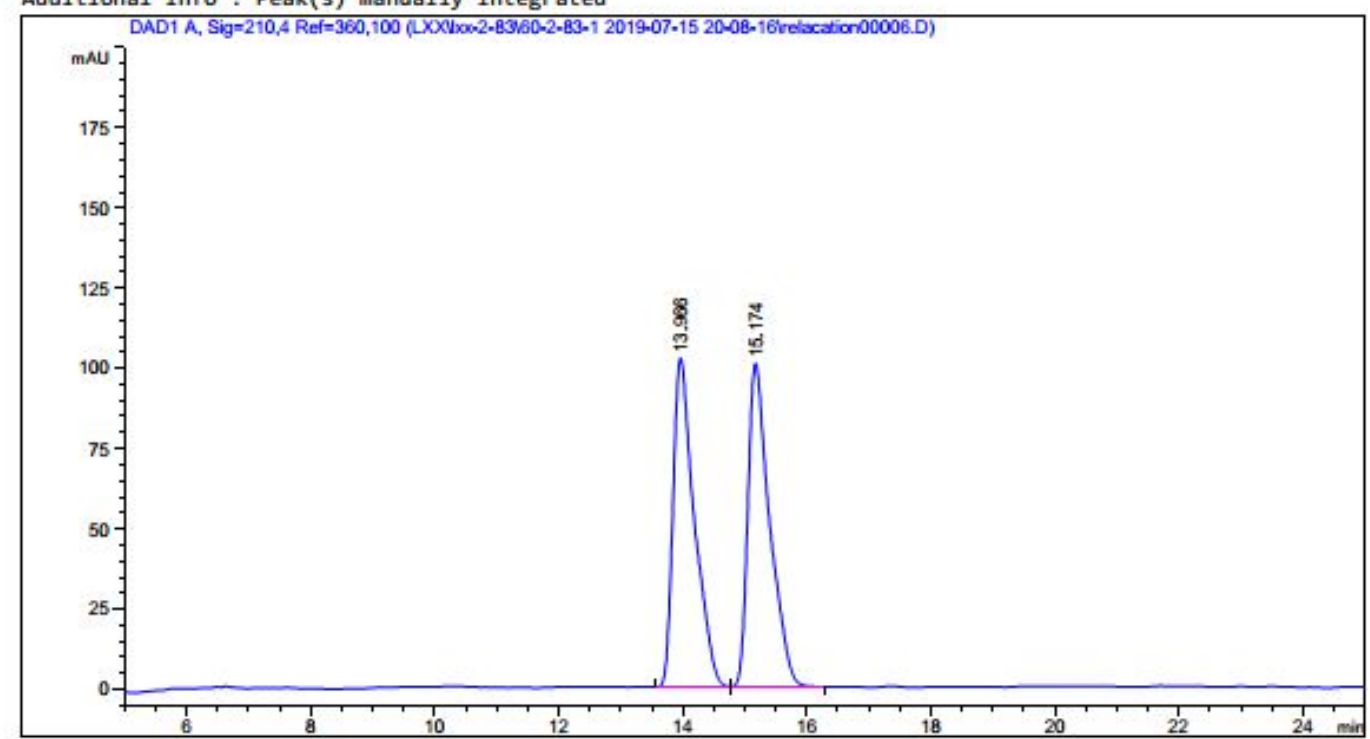

Area Percent Report

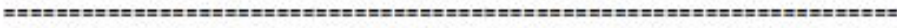

Sorted By : : Signal

Multiplier : $\quad 1.0000$

Dilution : 1.0000

Do not use Multiplier \& Dilution Factor with ISTDs

Signal 1: DAD1 A, Sig $=210,4$ Ref $=360,100$

\begin{tabular}{|c|c|c|c|c|c|}
\hline $\begin{array}{c}\text { Peak } \\
\#\end{array}$ & $\begin{array}{l}\text { RetTime Type } \\
\text { [min] }\end{array}$ & $\begin{array}{l}\text { Width } \\
\text { [min] }\end{array}$ & $\begin{array}{c}\text { Area } \\
{\left[\mathrm{mAU}^{*} \mathrm{~s}\right]}\end{array}$ & $\begin{array}{l}\text { Height } \\
\text { [mAU] }\end{array}$ & $\begin{array}{c}\text { Area } \\
\quad \%\end{array}$ \\
\hline$\cdots$ & $|-\ldots+|-\mid$ & (n........ & | & 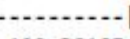 & .............. \\
\hline 1 & $13.966 \mathrm{BB}$ & 0.3477 & 2436.56348 & 102.38185 & 49.8180 \\
\hline 2 & $15.174 \mathrm{BB}$ & 0.3545 & 2454.36572 & 100.63210 & $50.182 \theta$ \\
\hline Tot: & 1s: & & $4890.9292 \theta$ & 203.01395 & \\
\hline
\end{tabular}




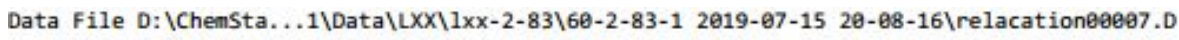
Sample Name: $83-1$

\begin{tabular}{|c|c|c|c|}
\hline $\begin{array}{l}\text { Acq. Operator } \\
\text { Sample Operator }\end{array}$ & $\begin{array}{l}\text { : SYSTEM } \\
: \text { SYSTEM }\end{array}$ & Seq. Line : & 7 \\
\hline Acq. Instrument & : LC & Location : & P2-B-64 \\
\hline Injection Date & : 7/15/2019 9:53:49 PM & Inj & 1 \\
\hline
\end{tabular}

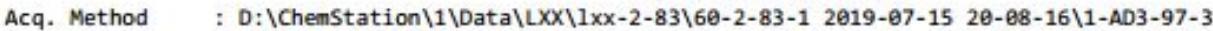
-1 ML-2UL-25C-25MIN.M

Last changed : 11/28/2018 10:55:29 AM by SYSTEM

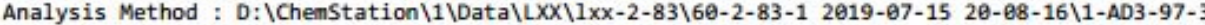

-1 ML-2UL-25C-25MIN.M (Sequence Method)

Last changed : 7/16/2019 9:08:56 AM by SYSTEM

(modified after loading)

Additional Info: Peak(s) manually integrated

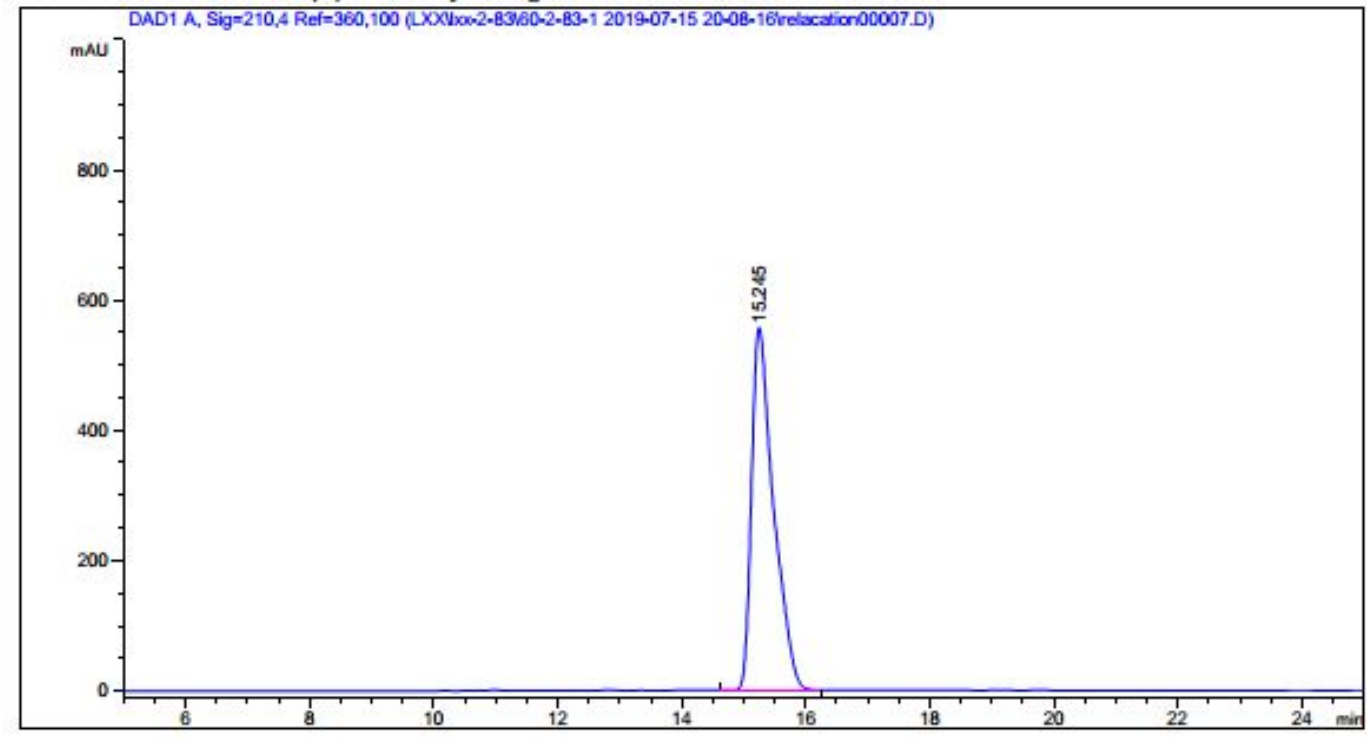

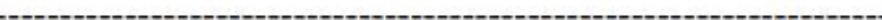

Area Percent Report

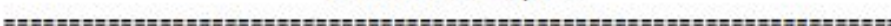

$\begin{array}{lll}\text { Sorted By } & : & \text { Signal } \\ \text { Multiplier } & : & 1.0000 \\ \text { Dilution } & : & 1.0000\end{array}$

Do not use Multiplier \& Dilution Factor with ISTDs

Signal 1: DAD1 A, Sig=210,4 Ref=360, 100

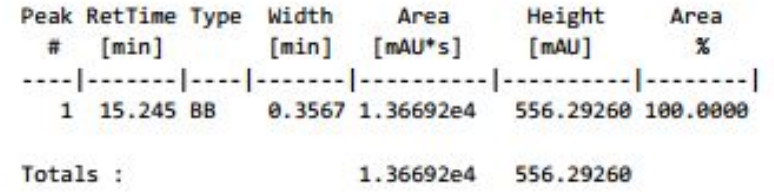


<smiles>C[C@@H]1Cc2ccccc2C2(C=CC(=O)CC2)C1</smiles>

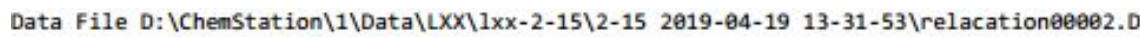
Sample Name: six-Rac

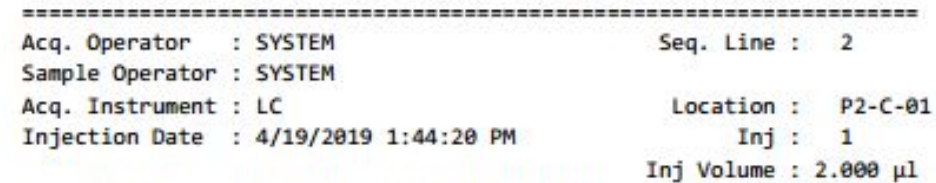

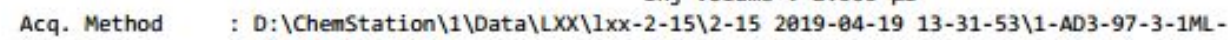
$2 \mathrm{UL}-25 \mathrm{C}-45 \mathrm{MIN} . \mathrm{M}$

Last changed : 4/19/2019 1:30:03 PM by SYSTEM

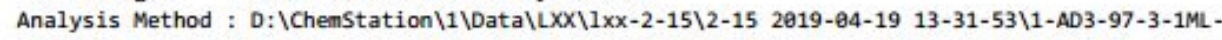

2UL-25C-45MIN.M (Sequence Method)

Last changed : 7/5/2019 10:07:01 AM by SYSTEM

(modified after loading)

Additional Info : Peak(s) manually integrated

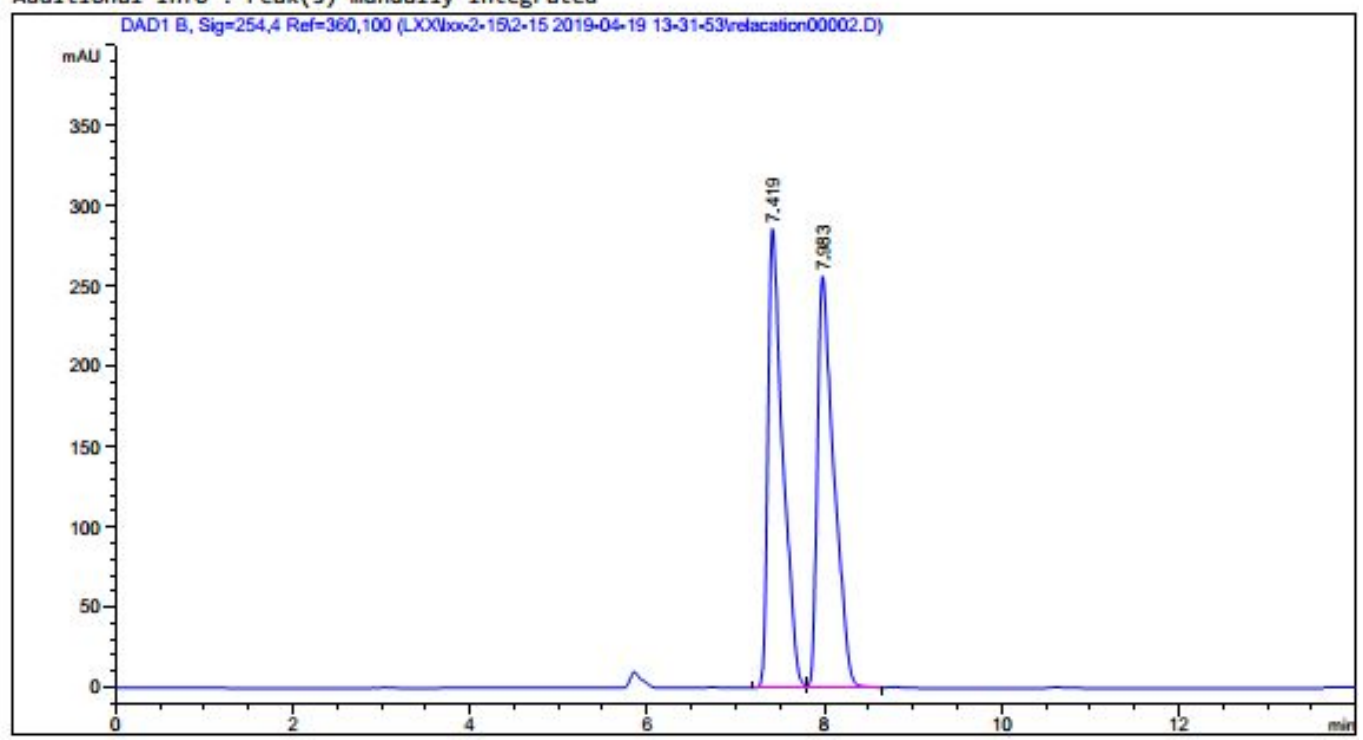

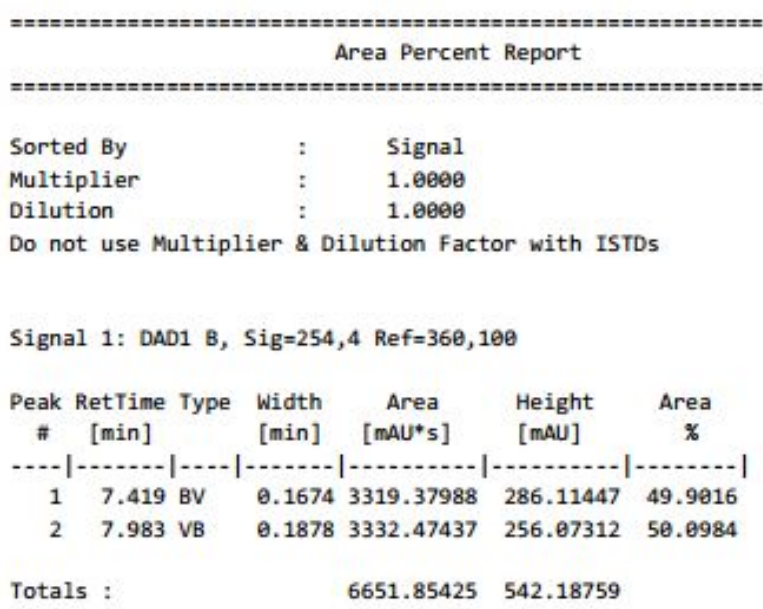




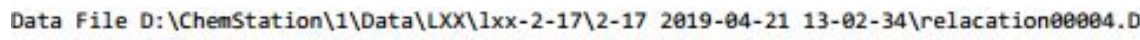
Sample Name: 17-3

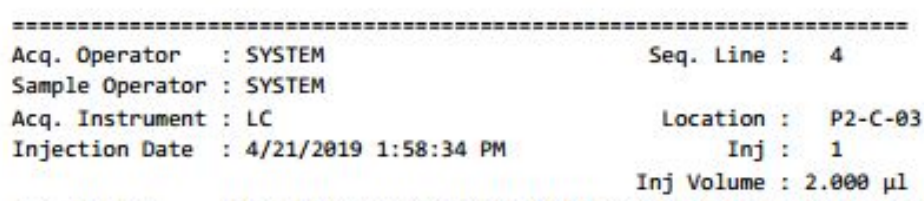

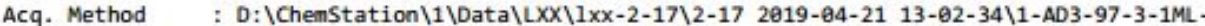
2UL-25C-25MIN.M

Last changed : 4/21/2019 1:17:06 PM by SYSTEM

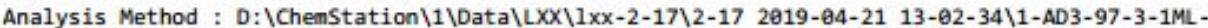
2UL-25C-25MIN.M (Sequence Method)

Last changed : 7/5/2019 10:17:07 AM by SYSTEM

(modified after loading)

Additional Info: Peak(s) manually integrated

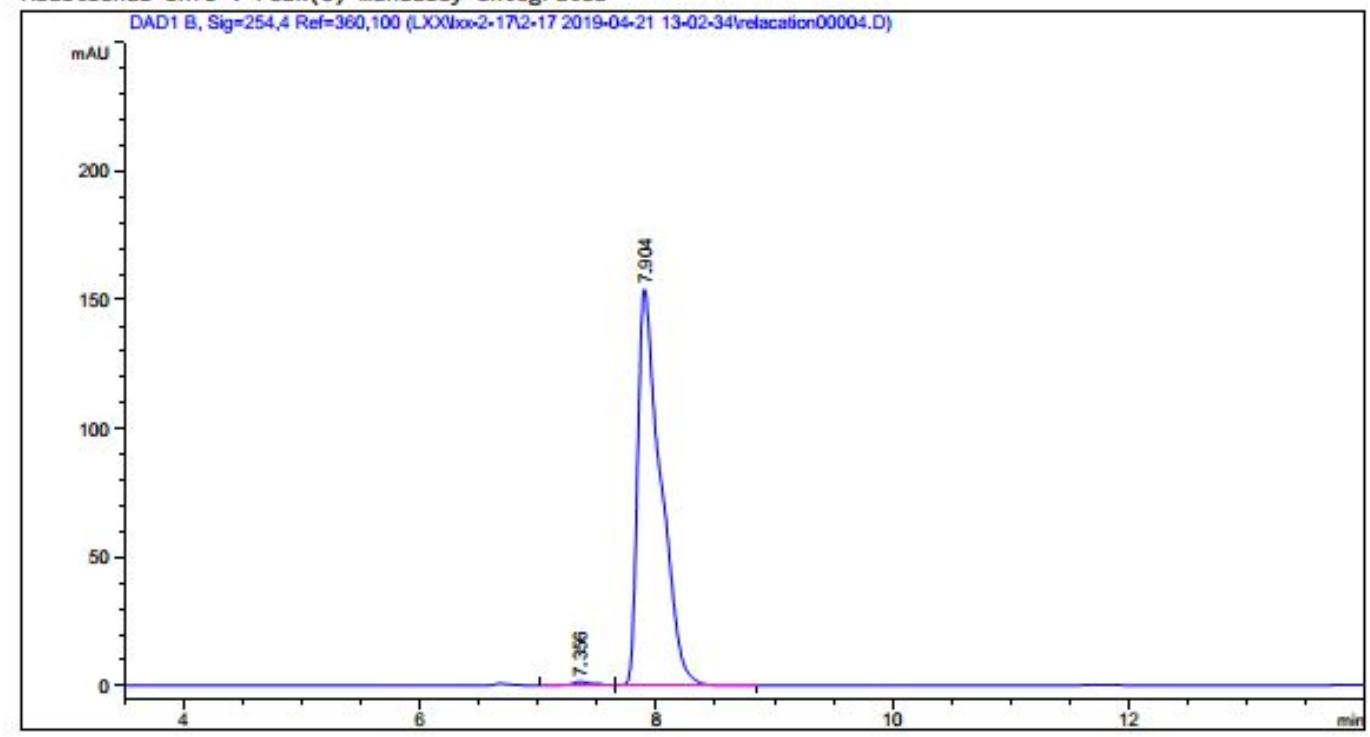

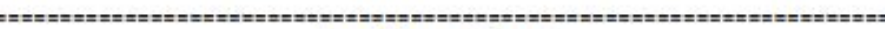

Area Percent Report

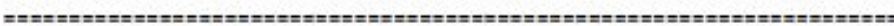

Sorted By : : Signal

Multiplier : : $1 . \theta 00 \theta$

Dilution : 1.0000

Do not use Multiplier \& Dilution Factor with ISTDs

Signal 1: DAD1 B, Sig $=254,4$ Ref $=360,100$

\begin{tabular}{|c|c|c|c|c|c|c|}
\hline $\begin{array}{c}\text { Peak } \\
\#\end{array}$ & $\begin{array}{l}\text { RetTime } \\
\text { [min] }\end{array}$ & Type & $\begin{array}{l}\text { Width } \\
\text { [min] }\end{array}$ & $\begin{array}{c}\text { Area } \\
{\left[\mathrm{mAU}^{*} \mathrm{~s}\right]}\end{array}$ & $\begin{array}{l}\text { Height } \\
\text { [mAU] }\end{array}$ & $\begin{array}{c}\text { Area } \\
\%\end{array}$ \\
\hline$\cdots-1$ & $|\cdots| \cdot \mid$ & & -....... & | & (n........... & ............. \\
\hline 1 & 7.356 & BB & 0.1667 & 18.42054 & 1.57262 & 0.8784 \\
\hline 2 & 7.964 & BB & 0.1915 & 2078.69482 & 153.93063 & 99.1216 \\
\hline Total & 15 : & & & 2097.11536 & 155.50325 & \\
\hline
\end{tabular}

LC 7/5/2019 10:17:14 AM SYSTEM 
<smiles>O=C1[CH]CCC2(C=C1)Cc1ccc(Cl)cc12</smiles>

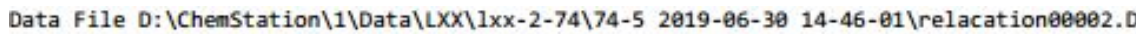
Sample Name: six-Cl-Rac
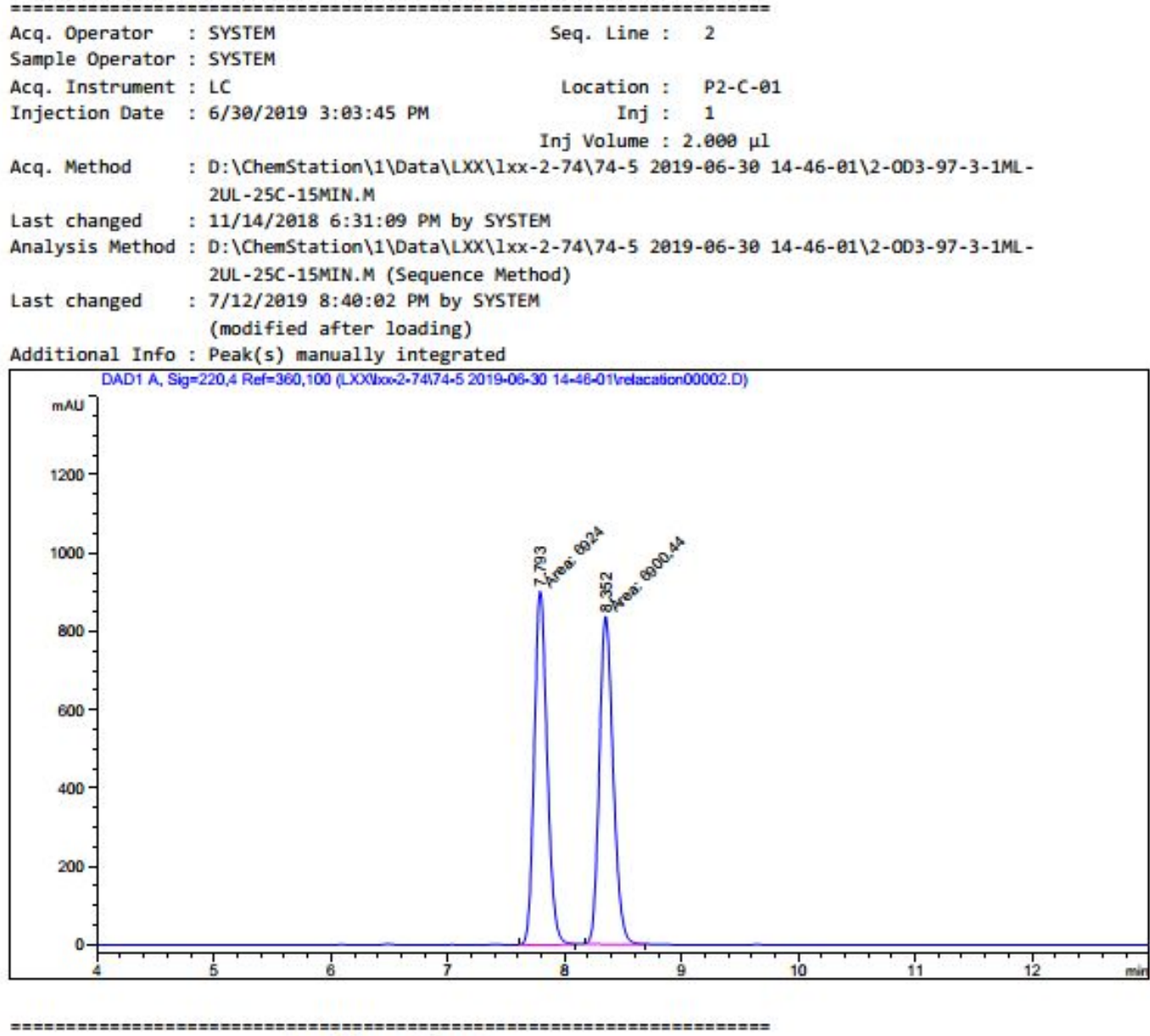

Area Percent Report

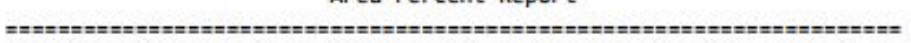

$\begin{array}{lll}\text { Sorted By } & : & \text { Signal } \\ \text { Multiplier } & : & 1.0000 \\ \text { Dilution } & : & 1.0000\end{array}$

Do not use Multiplier \& Dilution Factor with ISTDs

Signal 1: DAD1 A, Sig $=220,4$ Ref $=360,100$

\begin{tabular}{cccccc}
$\begin{array}{c}\text { Peak RetTime Type } \\
\text { \# } \\
\text { [min] }\end{array}$ & $\begin{array}{c}\text { Width } \\
\text { [min] }\end{array}$ & $\begin{array}{c}\text { Area } \\
\text { [mAU*s] }\end{array}$ & $\begin{array}{c}\text { Height } \\
\text { [mAU] }\end{array}$ & $\begin{array}{c}\text { Area } \\
\%\end{array}$ \\
\hline 1 & $7.793 \mathrm{MM}$ & 0.1275 & 6924.06439 & 905.17261 & 50.0852 \\
2 & $8.352 \mathrm{MM}$ & 0.1374 & 6960.44385 & 837.02625 & 49.9148
\end{tabular}




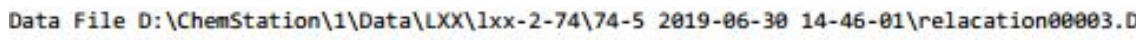
Sample Name: 74-5

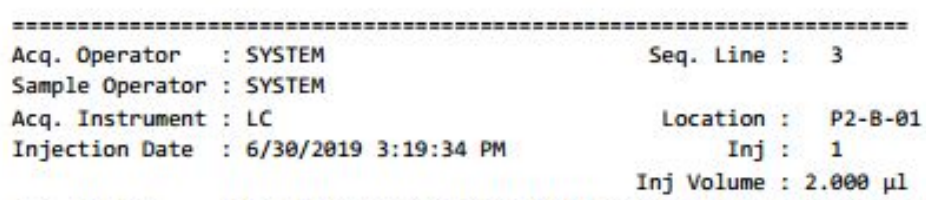

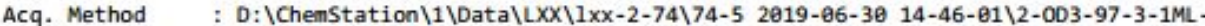
2UL-25C-15MIN. M

Last changed : 11/14/2018 6:31:09 PM by SYSTEM

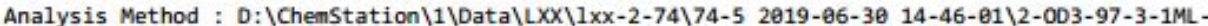
2UL-25C-15MIN.M (Sequence Method)

Last changed : 7/12/2019 8:40:50 PM by SYSTEM

(modified after loading)

Additional Info: Peak(s) manually integrated

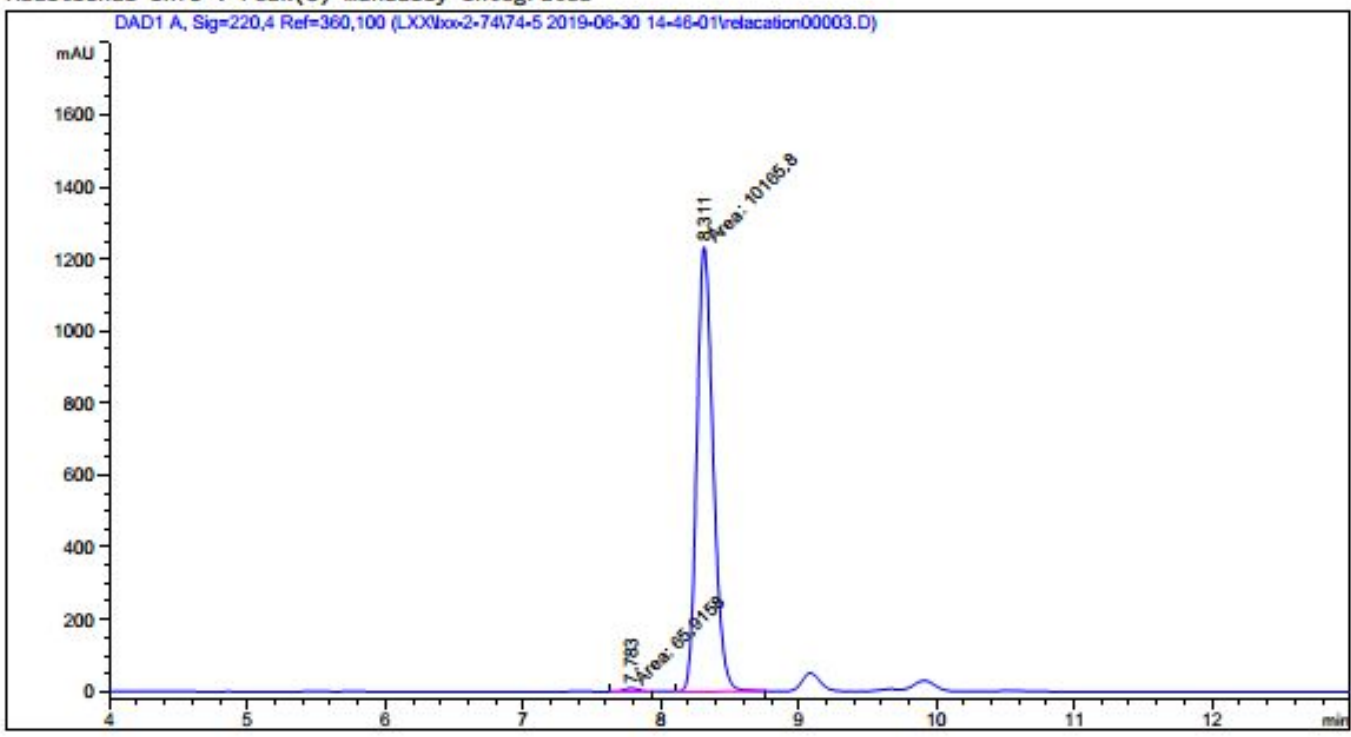

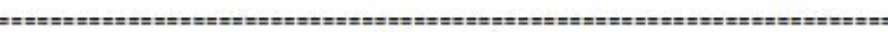

Area Percent Report

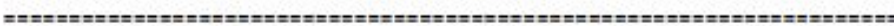

\section{Sorted By : : Signal}

Multiplier : : $1 . \theta 00 \theta$

Dilution : 1.0000

Do not use Multiplier \& Dilution Factor with ISTDs

Signal 1: DAD1 A, $S i g=220,4$ Ref $=360,100$

$$
\begin{aligned}
& \text { Peak RetTime Type Width Area Height Area } \\
& \text { \# [min] [min] [mAU*s] [mAU] } \% \\
& \text { - } \\
& \begin{array}{llllll}
1 & 7.783 \mathrm{MM} & 0.1236 & 65.91581 & 8.88676 & 0.6442
\end{array} \\
& \begin{array}{llllll}
2 & 8.311 \mathrm{MM} & 0.1373 & 1.01658 \mathrm{e} & 1233.81860 & 99.3558
\end{array} \\
& \begin{array}{lll}
\text { Totals : } & 1.02317 \mathrm{e} 4 & 1242.70537
\end{array}
\end{aligned}
$$

LC 7/12/2019 8:40:53 PM SYSTEM 
<smiles>O=C1C=CC2(CC1)CC[C@@H]1Cc3sccc3C12</smiles>

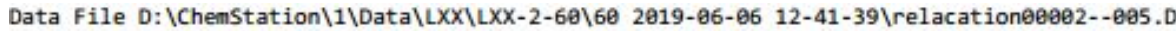
Sample Name: 60-1-RAC

\begin{tabular}{|c|c|c|c|}
\hline $\begin{array}{l}\text { Acq. Operator } \\
\text { Sample Operator }\end{array}$ & $\begin{array}{l}\text { : SYSTEM } \\
\text { : SYSTEM }\end{array}$ & Seq. Line : & 5 \\
\hline Acq. Instrument & : LC & Location : & P2-C-61 \\
\hline Injection Date & : 6/6/2019 1:34:06 PM & $\begin{array}{r}\text { Inj : } \\
\text { Inj Volume : }\end{array}$ & $\begin{array}{cc}: & 1 \\
: & 2.000 \mu 1\end{array}$ \\
\hline
\end{tabular}

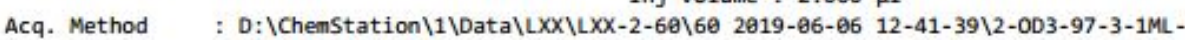
2UL-25C-30MIN. M

Last changed : 6/6/2019 1:55:59 PM by SYSTEM (modified after loading)

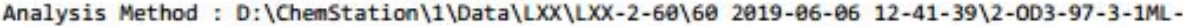
2UL-25C-30MIN.M (Sequence Method)

Last changed : 7/5/2019 3:12:13 PM by SYSTEM (modified after loading)

Additional Info: Peak(s) manually integrated

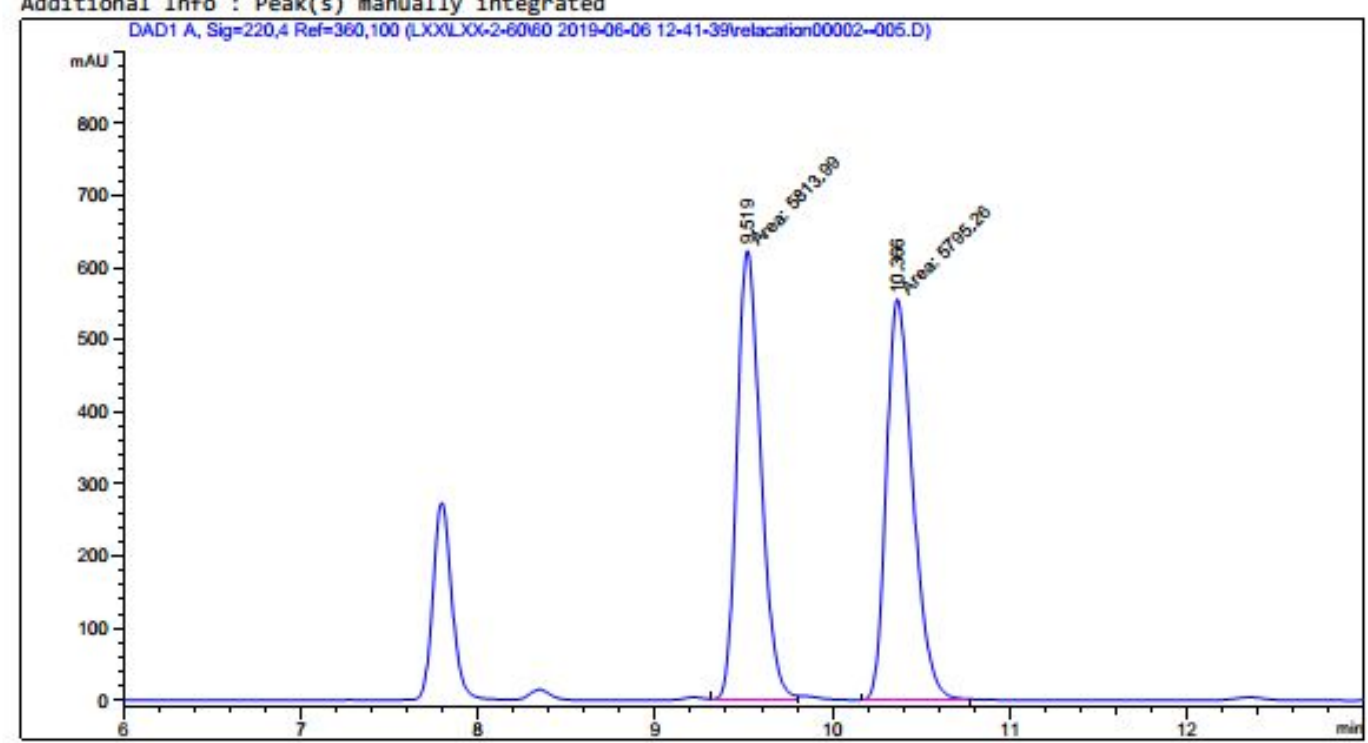

Area Percent Report

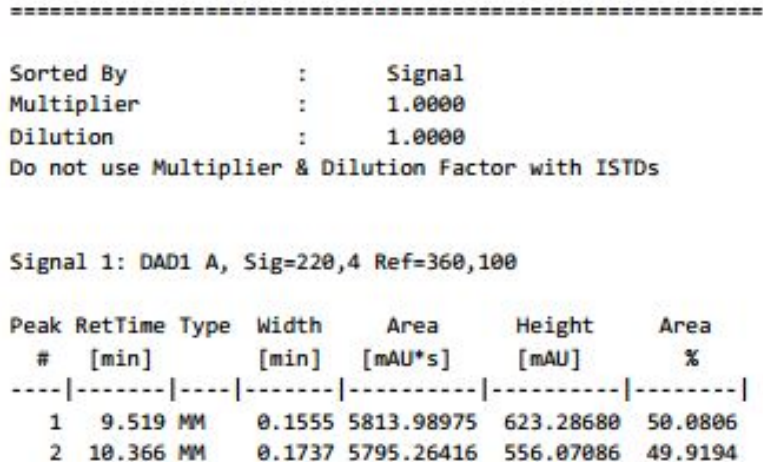




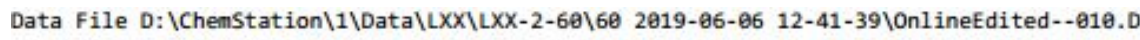
Sample Name: 60-1

\begin{tabular}{|c|c|c|c|}
\hline $\begin{array}{l}\text { Acq. Operator } \\
\text { Sample Operator }\end{array}$ & $\begin{array}{l}\text { : SYSTEM } \\
\text { : SYSTEM }\end{array}$ & Seq. Line & $: 10$ \\
\hline Acq. Instrument & : LC & Location & P2-B-61 \\
\hline Injection Date & : 6/6/2019 3:22:06 PM & $\begin{array}{r}\text { Inj } \\
\text { Inj Volume }\end{array}$ & $\begin{array}{l}: \quad 1 \\
: 2.009 \mu 1\end{array}$ \\
\hline
\end{tabular}

Acq. Method : D:\ChemStation\1\Data\LXX\LXX-2-60\60 2919-96-06 12-41-39\2-0D3-97-3-1ML2UL - 25C-3OMIN.M

Last changed : 6/6/2019 $3: 22: 27$ PM by SYSTEM (modified after loading)

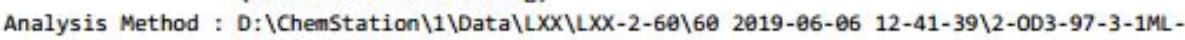
2UL-25C-30MIN.M (Sequence Method)

Last changed : 7/24/2019 5:36:03 PM by SYSTEM (modified after loading)

Additional Info: Peak(s) manually integrated

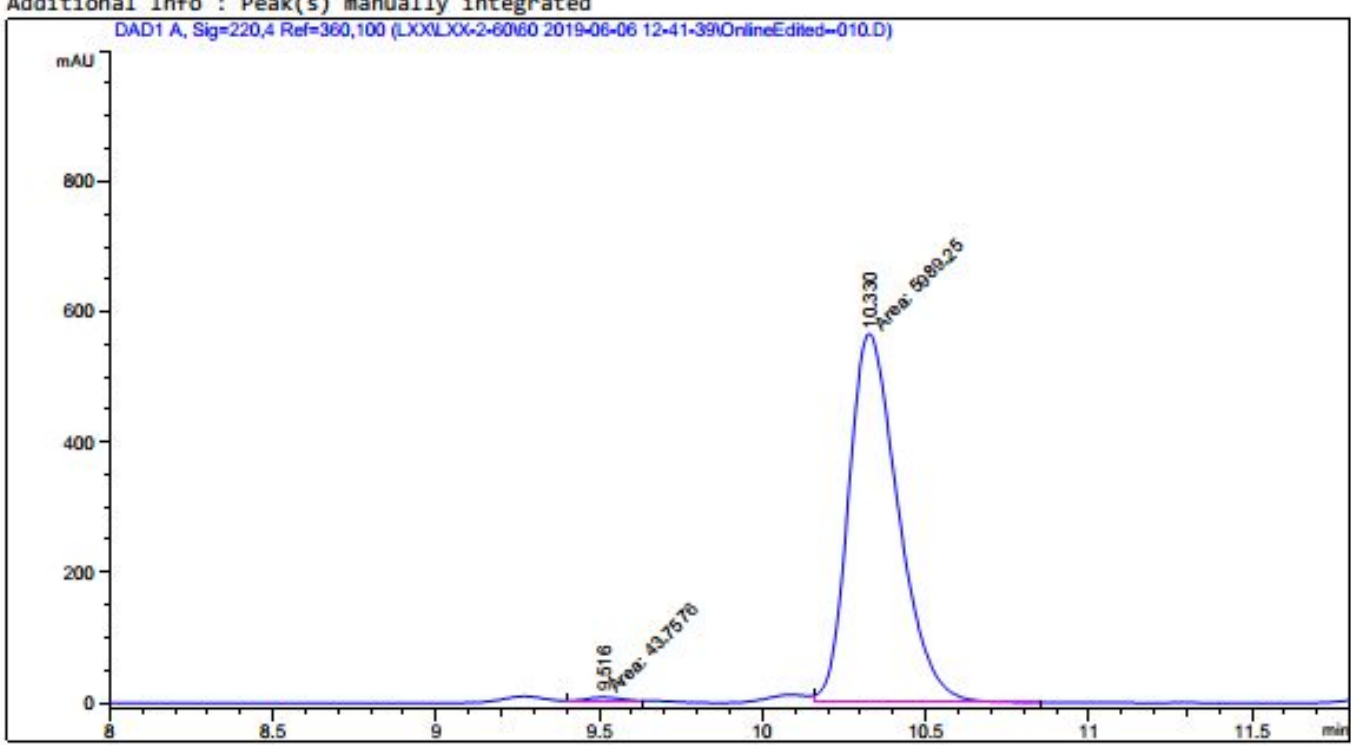

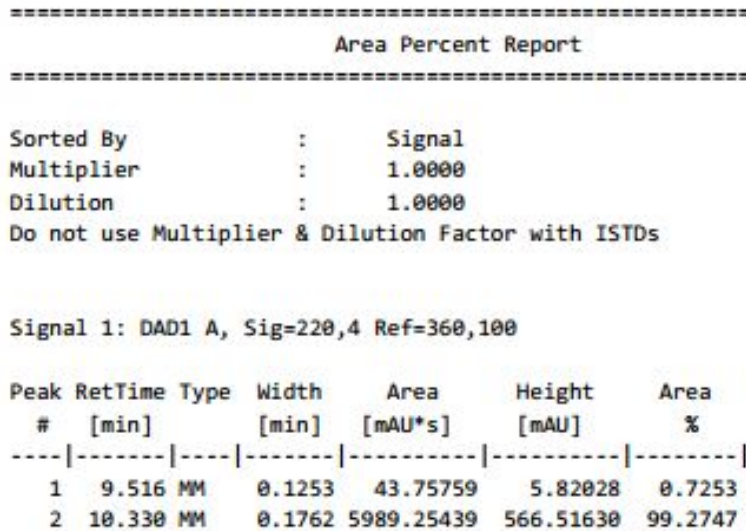


<smiles>O=C1C=CC2(CCOc3ccccc32)CC1</smiles>

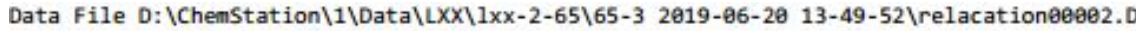
Sample Name: six-0-Rac

\begin{tabular}{|c|c|c|c|}
\hline $\begin{array}{l}\text { Acq. Operator } \\
\text { Sample Operator }\end{array}$ & $\begin{array}{l}\text { : SYSTEM } \\
: \text { SYSTEM }\end{array}$ & Seq. Line & 2 \\
\hline Acq. Instrument & : LC & Location & P2-C-61 \\
\hline Injection Date & : 6/20/2019 2:07:42 PM & Inj & $: \quad 1$ \\
\hline
\end{tabular}

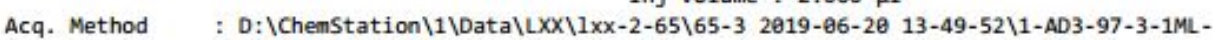
$2 \mathrm{UL}-25 \mathrm{C}-25 \mathrm{MIN}$. M

Last changed : 6/20/2019 2:31:22 PM by SYSTEM (modified after loading)

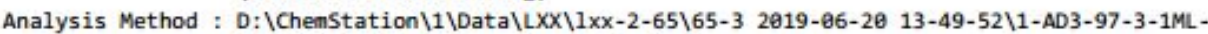
2UL-25C-25MIN.M (Sequence Method)

Last changed : 7/8/2019 2:25:08 PM by SYSTEM (modified after loading)

Additional Info : Peak(s) manually integrated

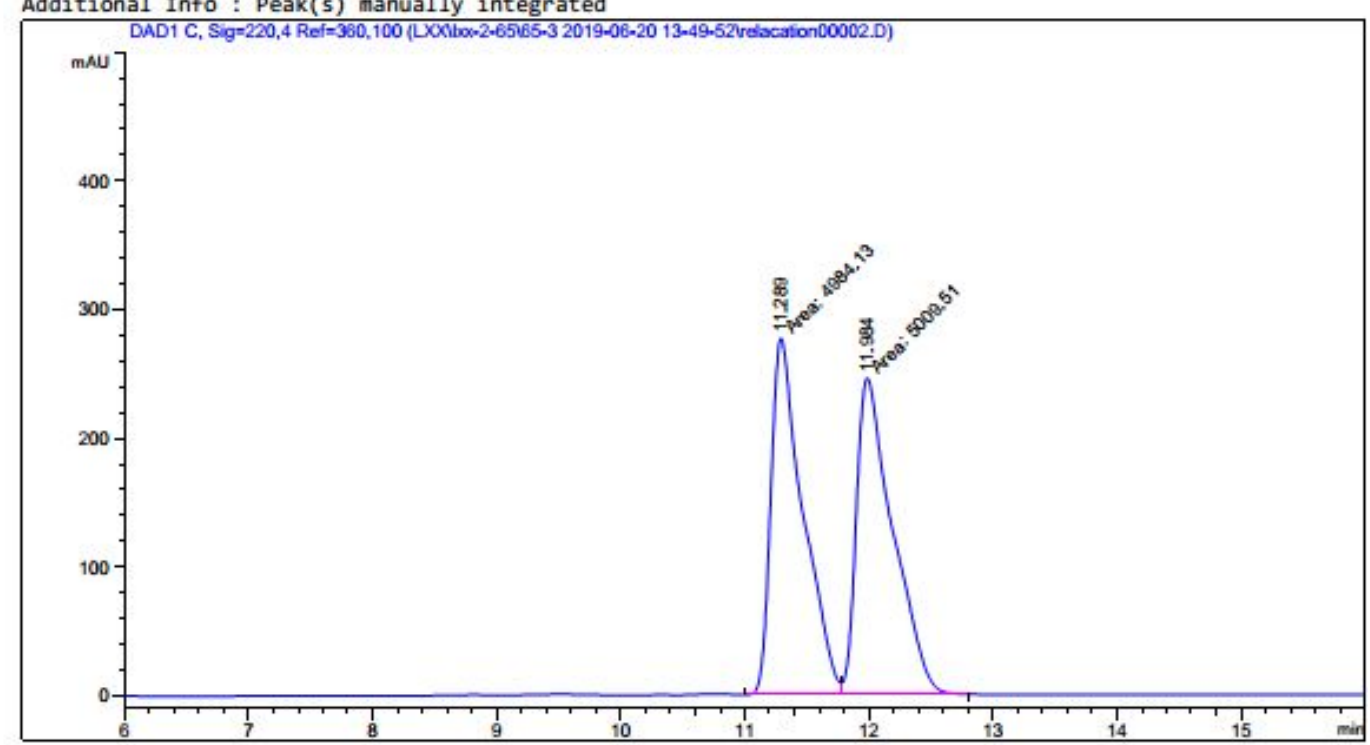

Area Percent Report

\begin{tabular}{|c|c|c|c|c|}
\hline Sorted By & : & \multicolumn{3}{|l|}{ Signal } \\
\hline Multiplier & : & \multicolumn{3}{|l|}{1.0000} \\
\hline Dilution & : & \multicolumn{3}{|l|}{1.0000} \\
\hline \multicolumn{5}{|c|}{ Do not use Multiplier \& Dilution Factor with ISTDs } \\
\hline \multicolumn{5}{|c|}{ Signal 1: DAD1 C, Sig=220,4 Ref $=360,100$} \\
\hline $\begin{array}{l}\text { Peak RetTime Type } \\
\# \quad \text { [min] }\end{array}$ & $\begin{array}{l}\text { Width } \\
\text { [min] }\end{array}$ & $\begin{array}{c}\text { Area } \\
\text { [mAU*s] }\end{array}$ & $\begin{array}{l}\text { Height } \\
\text { [MAU] }\end{array}$ & $\begin{array}{c}\text { Area } \\
\text { \% }\end{array}$ \\
\hline$\ldots|-\ldots+|-1$ & $-\ldots-1$ & -.......... & $-2.2-1$ & $\ldots-1$ \\
\hline $111.289 \mathrm{MF}$ & 0.2998 & 4984.12793 & 277.06967 & 49.8730 \\
\hline $211.984 \mathrm{FM}$ & 0.3391 & 5009.51123 & 246.19238 & 50.1270 \\
\hline
\end{tabular}




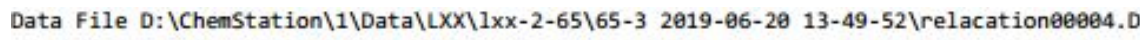
Sample Name: 65-3

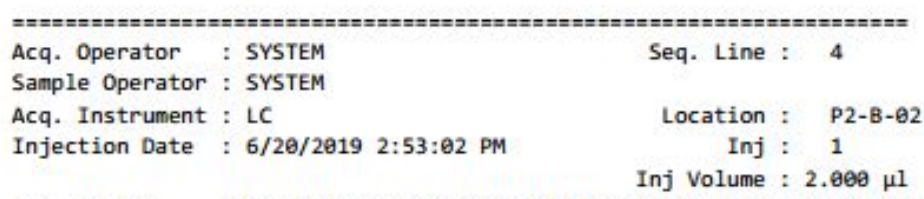

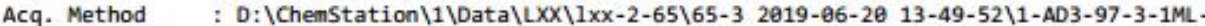
2UL-25C-25MIN. M

Last changed : 6/20/2019 2:31:22 PM by SYSTEM

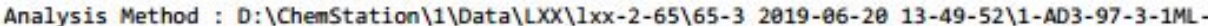
2UL-25C-25MIN.M (Sequence Method)

Last changed : 7/8/2019 2:21:53 PM by SYSTEM

(modified after loading)

Additional Info: Peak(s) manually integrated

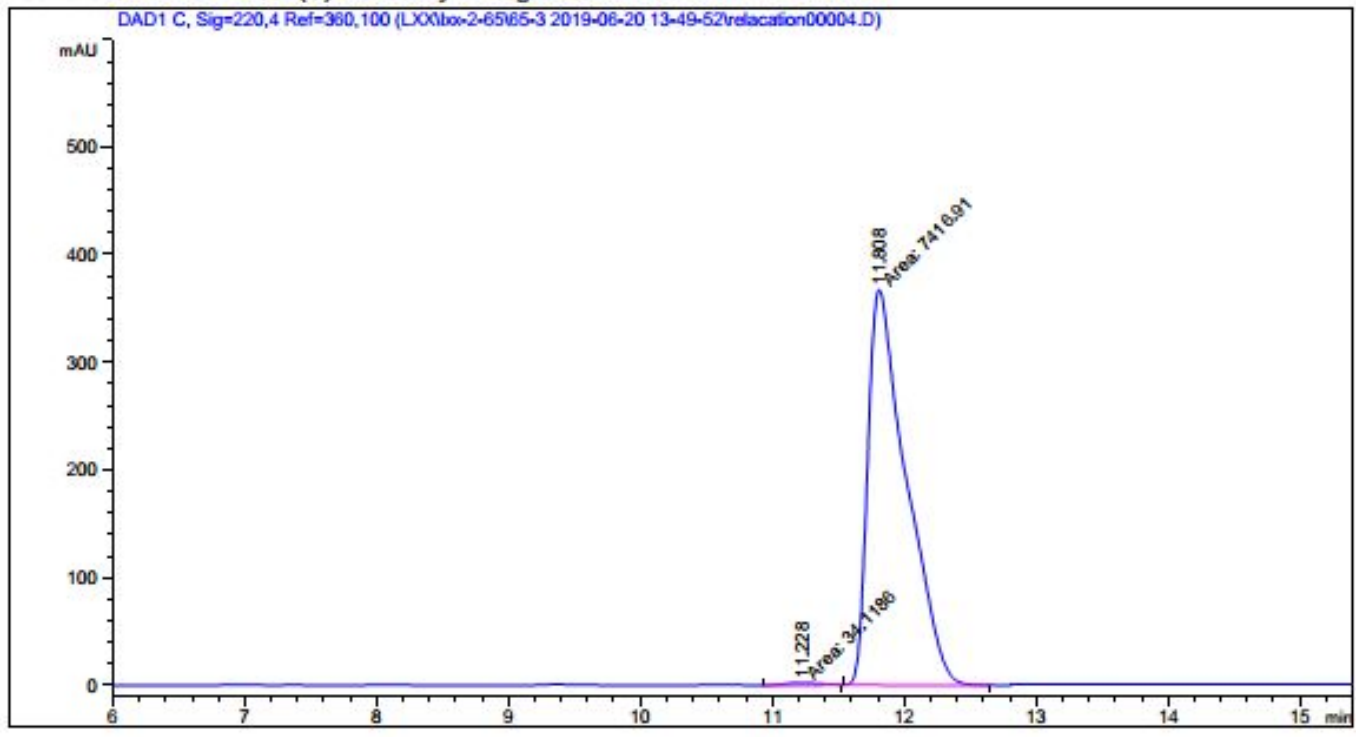

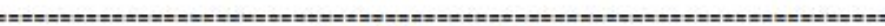

Area Percent Report

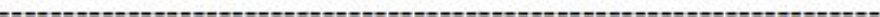

\section{Sorted By : : Signal}

Multiplier : : 1.000

Dilution : 1.0000

Do not use Multiplier \& Dilution Factor with ISTDs

Signal 1: DAD1 C, $S i g=220,4$ Ref $=360,100$

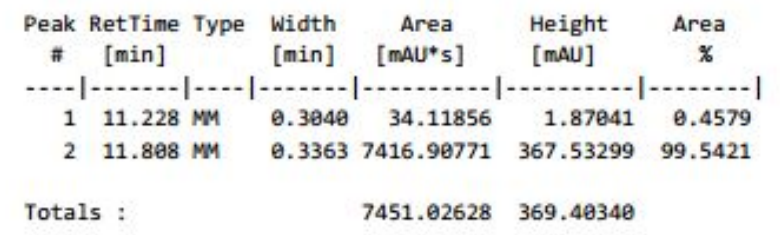

LC 7/8/2019 2:21:58 PM SYSTEM

Page 1 of 2 
<smiles>O=C1C=CC2(CCSc3ccccc32)CC1</smiles>

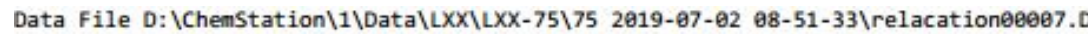
Sample Name: 75-2-Rac
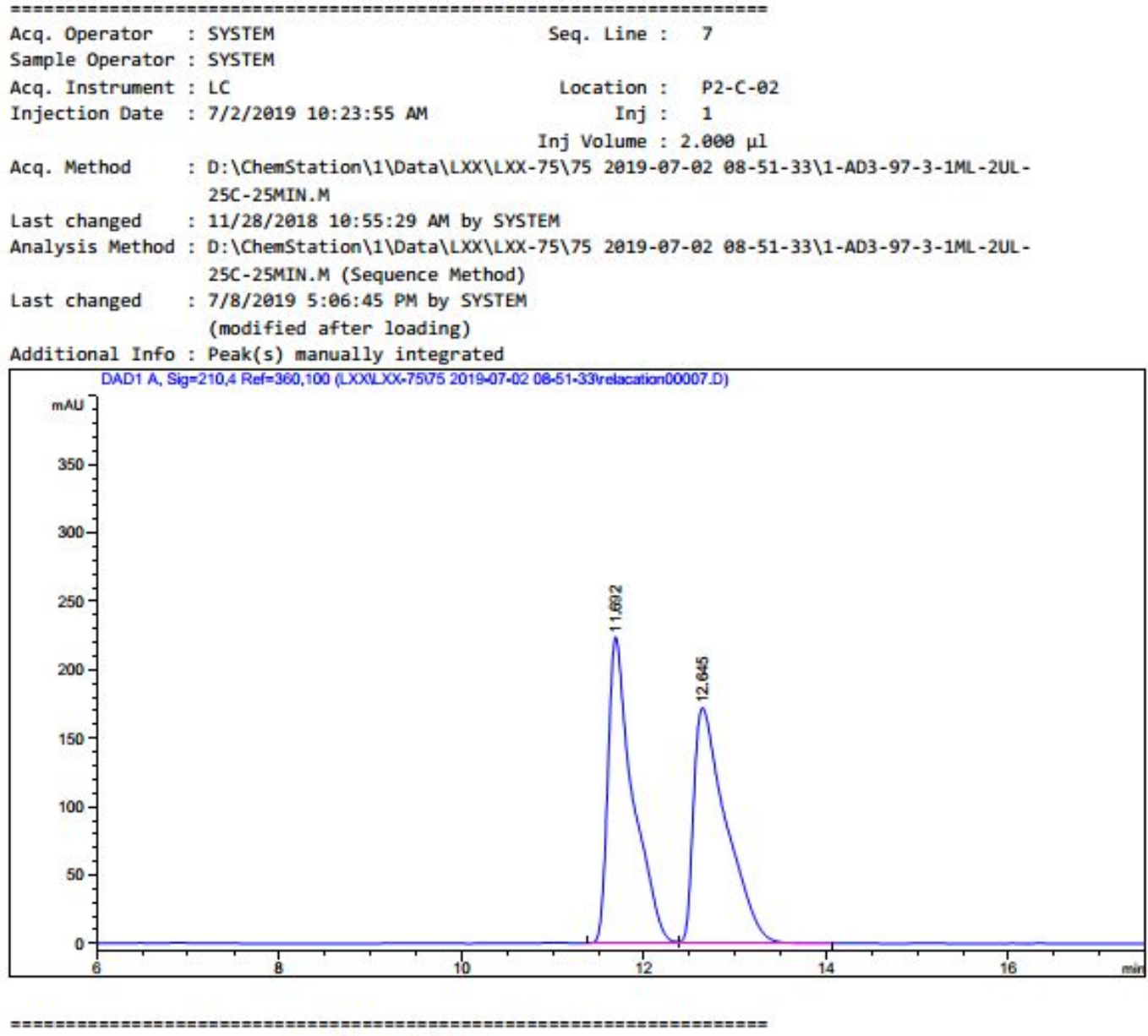

Area Percent Report

\begin{tabular}{|c|c|c|}
\hline Sorted By & : & Signal \\
\hline Multiplier & : & $1 . \theta 00 \theta$ \\
\hline Dilution & : & 1.0000 \\
\hline
\end{tabular}

Do not use Multiplier \& Dilution Factor with ISTDs

Signal 1: DAD1 A, $\mathrm{Sig}=210,4$ Ref $=360,100$

\begin{tabular}{|c|c|c|c|c|c|}
\hline $\begin{array}{c}\text { Peak } \\
\#\end{array}$ & $\begin{array}{l}\text { RetTime Type } \\
\text { [min] }\end{array}$ & $\begin{array}{l}\text { Width } \\
\text { [min] }\end{array}$ & $\begin{array}{c}\text { Area } \\
{[\mathrm{mAU} * \mathrm{~s}]}\end{array}$ & $\begin{array}{l}\text { Height } \\
\text { [mAU] }\end{array}$ & $\begin{array}{c}\text { Area } \\
\text { \% }\end{array}$ \\
\hline$\cdots$ & $|-\ldots+|-\mid$ & -..... & - & . & -....... \\
\hline 1 & $11.692 \mathrm{BV}$ & 0.2675 & 4244.08154 & 223.53853 & 49.8786 \\
\hline 2 & $12.645 \mathrm{vB}$ & 0.3554 & 4264.74365 & 171.93437 & 50.1214 \\
\hline \multicolumn{3}{|c|}{ Totals : } & 8508.82520 & 395.47290 & \\
\hline
\end{tabular}




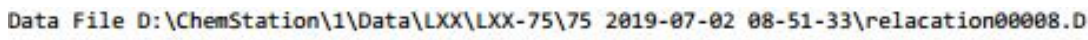
Sample Name: $75-2$

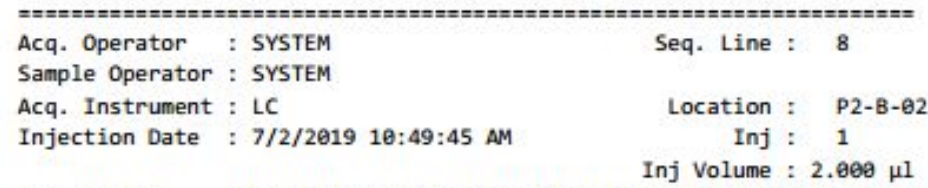

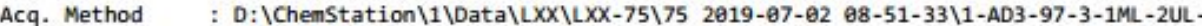
$25 C-25$ MIN. M

Last changed : 7/2/2019 11:10:26 AM by SYSTEM (modified after loading)

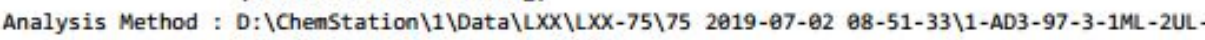
25C-25MIN.M (Sequence Method)

Last changed : 7/8/2019 5:04:59 PM by SYSTEM (modified after loading)

Additional Info: Peak(s) manually integrated

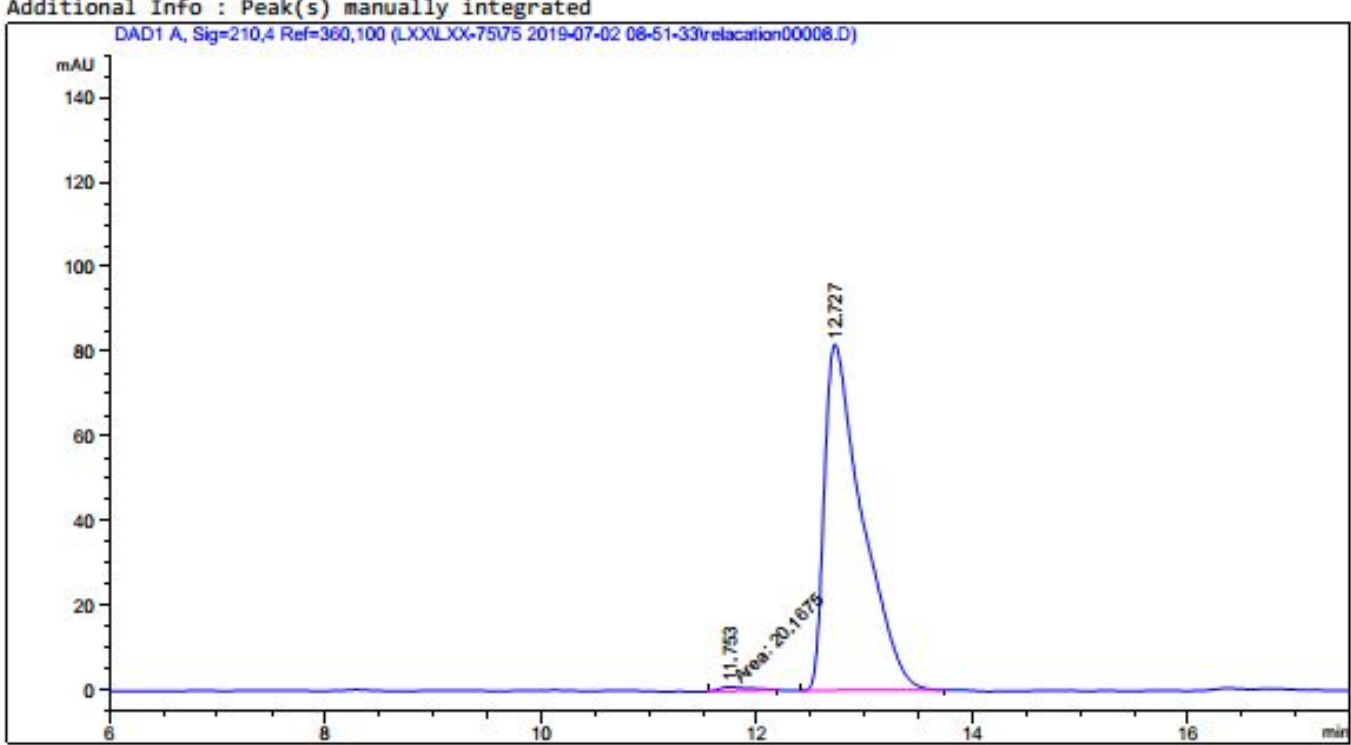

\begin{tabular}{|c|c|c|}
\hline Sorted By & : & Sigr \\
\hline Multiplier & : & $1 . \theta$ \\
\hline Dilution & : & 1.6 \\
\hline
\end{tabular}

Do not use Multiplier \& Dilution Factor with ISTDs

Signal 1: DAD1 A, Sig $=210,4$ Ref $=360,100$

\begin{tabular}{|c|c|c|c|c|c|c|}
\hline $\begin{array}{c}\text { Peak } \\
\#\end{array}$ & $\begin{array}{l}\text { RetTime } \\
\text { [min] }\end{array}$ & Type & $\begin{array}{l}\text { Width } \\
\text { [min] }\end{array}$ & $\begin{array}{c}\text { Area } \\
{\left[\mathrm{mAU}^{*} \mathrm{~s}\right]}\end{array}$ & $\begin{array}{l}\text { Height } \\
\text { [mAU] }\end{array}$ & $\begin{array}{c}\text { Area } \\
\text { \% }\end{array}$ \\
\hline & & & & . & & \\
\hline 1 & 11 & M & 0.3 & 20.16748 & $9.95974 e-1$ & 3066 \\
\hline 2 & 12.727 & $B B$ & .3471 & 1984.46851 & 81.79012 & 98.9946 \\
\hline
\end{tabular}


<smiles>CCC12C=CC(=O)[C@H](CCc3ccccc31)CC2</smiles>

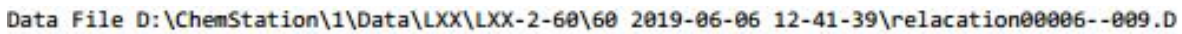
Sample Name: 60-5-RAC

\begin{tabular}{|c|c|c|c|}
\hline $\begin{array}{l}\text { Acq. Operator } \\
\text { Sample Operator }\end{array}$ & $\begin{array}{l}: \text { SYSTEM } \\
: \text { SYSTEM }\end{array}$ & Seq. Line & 9 \\
\hline Acq. Instrument & : LC & Location & $\mathrm{P} 2-\mathrm{C}-05$ \\
\hline Injection Date & : 6/6/2019 3:09:16 PM & $\begin{array}{r}\text { Inj } \\
\text { Inj Volume }\end{array}$ & $\begin{array}{l}: \quad 1 \\
: 2.000 \mu 1\end{array}$ \\
\hline
\end{tabular}

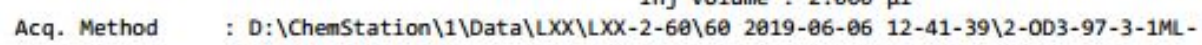
2UL-25C-3eMIN. M

Last changed : 6/6/2019 3:17:35 PM by SYSTEM

(modified after loading)

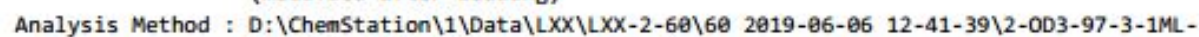

2UL-25C-30MIN.M (Sequence Method)

Last changed : 7/12/2019 $8: 46: 48$ PM by SYSTEM

(modified after loading)

\begin{tabular}{l} 
Additional Info : Peak(s) manually integrated \\
\hline DAD1 A, Sig=220,4 Ref $=360,100$ (LXXLXX.2-60660 2019-06-06 12-41-39velacation00006-009.D)
\end{tabular}

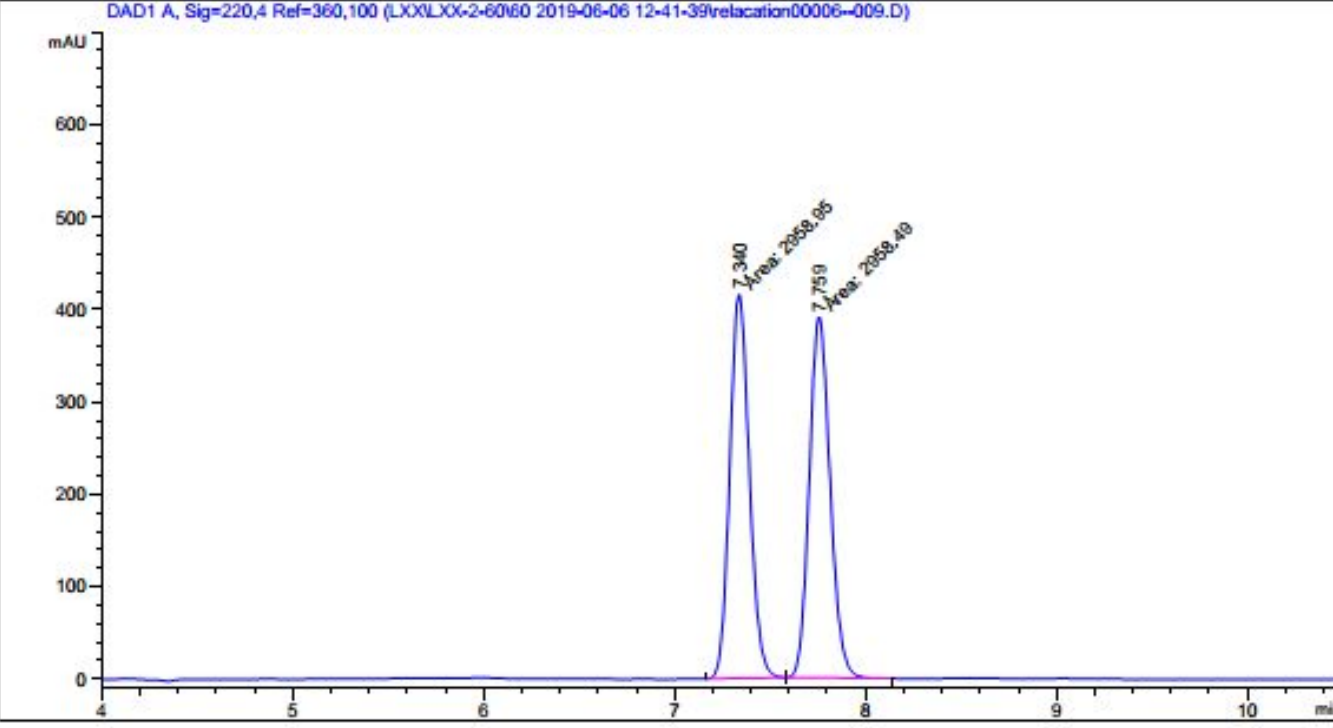

Area Percent Report

\begin{tabular}{|c|c|c|c|c|}
\hline Sorted By & : & Signal & & \\
\hline Multiplier & : & 1.0000 & & \\
\hline Dilution & : & 1.0000 & & \\
\hline \multicolumn{5}{|c|}{ Do not use Multiplier \& Dilution Factor with ISTDs } \\
\hline \multicolumn{5}{|c|}{ Signal 1: DAD1 A, Sig=220,4 Ref=360,100 } \\
\hline $\begin{array}{l}\text { Peak RetTime Type } \\
\# \quad \text { [min] }\end{array}$ & $\begin{array}{l}\text { Width } \\
\text { [min] }\end{array}$ & $\begin{array}{c}\text { Area } \\
{\left[\mathrm{mAU}^{*} \mathrm{~s}\right]}\end{array}$ & $\begin{array}{l}\text { Height } \\
{[\mathrm{mAU}]}\end{array}$ & $\begin{array}{l}\text { Area } \\
\text { \% }\end{array}$ \\
\hline$\cdots-1-n-1-2$ & 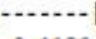 & $|-2.2-2 .-2|$ & $-2 .-2-1$ & $2-2 .-1$ \\
\hline $7.340 \mathrm{MM}$ & 0.1186 & 2958.95020 & 415.95755 & 50.0039 \\
\hline $27.759 \mathrm{MM}$ & 0.1261 & 2958.48779 & 391.13452 & 49.9961 \\
\hline
\end{tabular}


Data File D: \ChemStation\1\Data\LXX\LXX-2-60\60 2019-06-06 12-41-39\OnlineEdited--012.D Sample Name: $60-5$

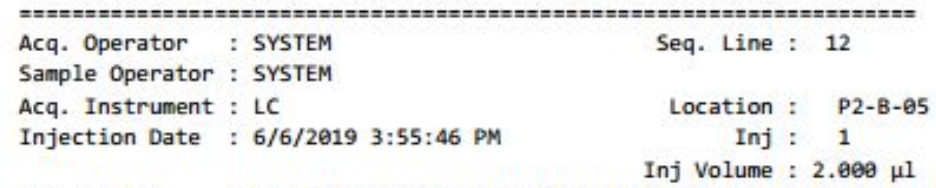

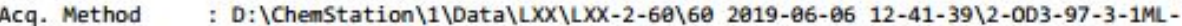
2UL - 25C-3eMIN. M

Last changed : 6/6/2019 $3: 22: 27$ PM by SYSTEM

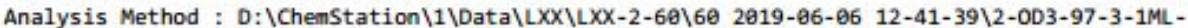
2UL-25C-30MIN.M (Sequence Method)

Last changed : 7/12/2019 8:44:15 PM by SYSTEM

(modified after loading)

Additional Info : Peak(s) manually integrated

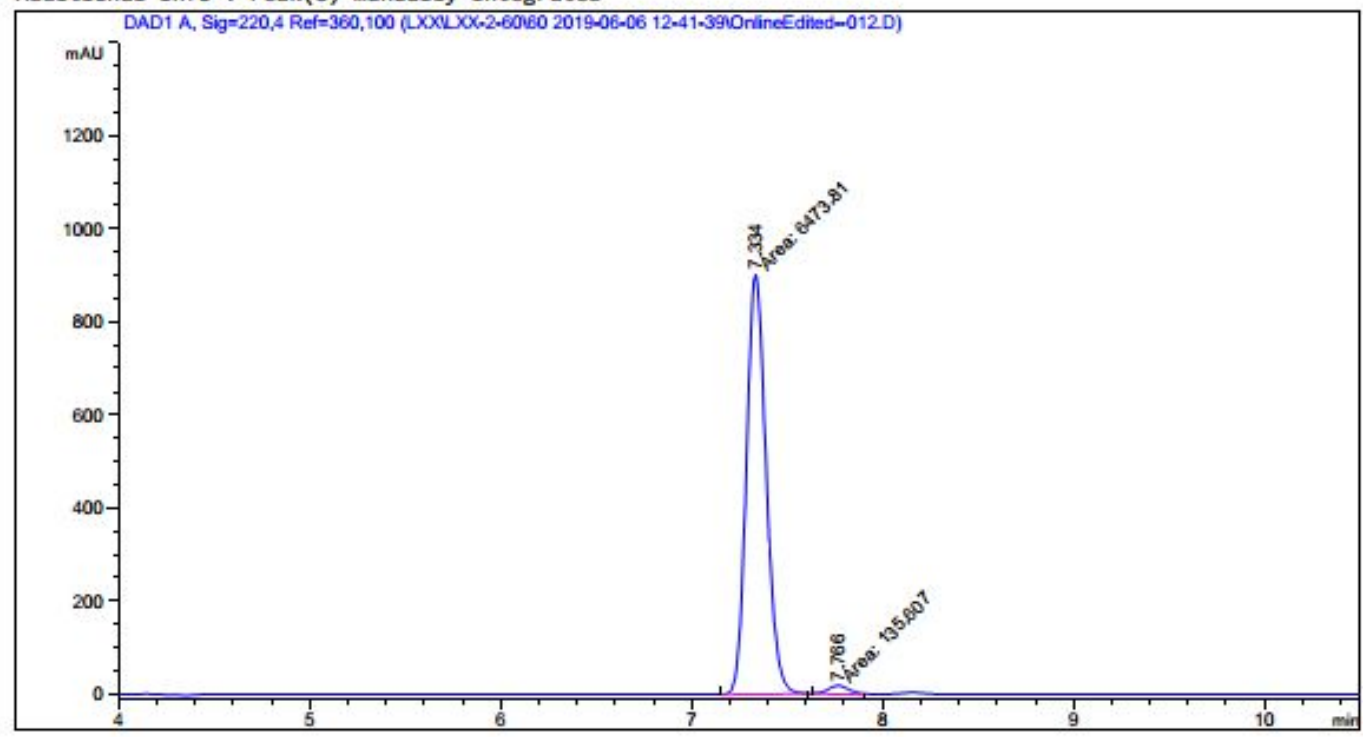

Area Percent Report

Sorted By : : Signal

Multiplier : $\quad 1.0000$

Dilution : 1.0000

Do not use Multiplier \& Dilution Factor with ISTDs

Signal 1: DAD1 A, Sig $=220,4$ Ref $=360,100$

\begin{tabular}{cccccc}
$\begin{array}{c}\text { Peak RetTime Type } \\
\text { [min] }\end{array}$ & $\begin{array}{c}\text { Width } \\
\text { [min] }\end{array}$ & $\begin{array}{c}\text { Area } \\
\text { [mAU*s] }\end{array}$ & $\begin{array}{l}\text { Height } \\
\text { [mAU] }\end{array}$ & $\begin{array}{c}\text { Area } \\
\%\end{array}$ \\
\hline 1 & $7.334 \mathrm{MM}$ & 0.1195 & 6473.81445 & 902.71191 & 97.9483 \\
2 & $7.766 \mathrm{MM}$ & 0.1287 & 135.60738 & 17.55724 & 2.0517 \\
& & & & & \\
Totals : & & 6609.42183 & 920.26916 &
\end{tabular}

